SLAC-R-1023

\title{
Study of Charmless Semileptonic $B$ Decays and a Measurement of the CKM Matrix Element | Vub | at BaBar
}

by

Gary Peter Taylor

April 2004 


\title{
Study of Charmless Semileptonic B Decays and a Measurement of the CKM Matrix Element $\left|V_{u b}\right|$ at $B A B A R$
}

\author{
Gary Peter Taylor \\ Imperial College London.
}

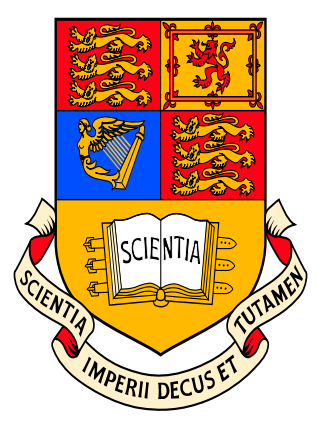

A thesis submitted for the degree of

Doctor of Philosophy

of The University of London

and the Diploma of Imperial College.

April, 2004 


\section{Abstract}

This thesis presents a measurement of exclusive charmless semileptonic branching fractions of $B$ mesons measured using $81.9 \mathrm{fb}^{-1}$ of data (approximately 90 million $B \bar{B}$ pairs) collected between 1999 and 2002 by the $B A B A R$ detector operating at the PEP-II $e^{+} e^{-}$storage ring, at SLAC. The measured branching fractions are

$$
\begin{aligned}
\mathcal{B}\left(B^{0} \rightarrow \pi^{-} l^{+} \nu\right) & =(1.67 \pm 0.11 \pm 0.07) \times 10^{-4} \\
\mathcal{B}\left(B^{0} \rightarrow \rho^{-} l^{+} \nu\right) & =\left(2.10 \pm 0.18_{-0.28}^{+0.23}\right) \times 10^{-4} \\
\mathcal{B}\left(B^{0} \rightarrow \omega l^{+} \nu\right) & =(1.69 \pm 0.26 \pm 0.24) \times 10^{-4}
\end{aligned}
$$

where the errors are statistical and systematic respectively. An estimate of the CKM matrix element $\left|V_{u b}\right|$ is extracted from these branching fractions using the ISGW2 theoretical calculations of the form factors. The results are

$$
\begin{aligned}
\left|V_{u b}\right|_{\pi} & =(3.36 \pm 0.11 \pm 0.07 \pm 0.84) \times 10^{-3} \\
\left|V_{u b}\right|_{\rho} & =\left(3.10 \pm 0.14_{-0.21}^{+0.17} \pm 0.76\right) \times 10^{-3}
\end{aligned}
$$

where the errors are statistical, systematic and theoretical respectively.

Also presented is work carried out to categorise and correct non-linearities in the response of the EMC readout electronics. 


\section{Acknowledgments}

First of all I would like to thank my supervisor Jordan Nash for his support throughout this PhD. A special thanks should also go to Paul Dauncey for his much appreciated help and advice. I would like to thank Julie Sedgbeer, John Hassard and Peter Dornan for giving me the opportunity to study at Imperial College. I acknowledge PPARC for funding this research.

SLAC is a great place to live and work and I enjoyed my time there immensely. This was in no small part due to the people I had the privilege to meet. I would like to single out Ivo Eschrich, Martin Kocian, Helmut Marsiske, Amanda Weinstein and Vera Lüth for their help throughout my time at SLAC.

Thanks to the following people for making the last three(ish) years that little bit more enjoyable:

- SLACers; Andy, Henning, Rich, Nick, Simon, Paul (Jack) and Manny.

- The Imperial Babar group; Dan, Geoff, Wahid, Jamie, Mike and Ulrik.

- The rest of the Imperial College crew; Matt, Ian, Rob, Steve, Chris, Rod, Alex, Dave, Kostas, Hugh and Michele.

Last but definitely not least, I'd like to thank my family for their support; and not just during the last three years.

Cheers all.

Gary Peter Taylor

London, April 2004 


\section{Contents}

Abstract 1

Acknowledgements 2

Contents 3

$\begin{array}{ll}\text { List of Figures } & 9\end{array}$

$\begin{array}{ll}\text { List of Tables } & 16\end{array}$

Chapter 1. Theory 21

1.1 Introduction 21

$\begin{array}{lll}1.2 & C P \text { Violation } & 22\end{array}$

1.2.1 Direct $C P$ Violation 23

1.2.2 Indirect $C P$ Violation 23

1.2.3 CP Violation in the Interference of Mixing and Decay 24

1.3 The Standard Electroweak Model 24

1.4 The CKM Matrix 26

1.4.1 The Unitarity Triangle 28

1.4.2 Constraining the CKM Matrix 29

1.5 Measuring $V_{u b}$ at $B A B A R \quad 34$

$\begin{array}{lll}\text { 1.5.1 Introduction } 34 & 34\end{array}$

1.5.2 Decay Kinematics 34

1.5.3 Theoretical Models for Form Factors 37

$\begin{array}{lll}\text { 1.5.4 Inclusive Measurements } & 39\end{array}$

1.5.5 Previous Measurements of $\left|V_{u b}\right| \quad 39$

$\begin{array}{lll}1.6 & \text { Current Analysis } & 39\end{array}$ 
Chapter 2. The PEP-II Storage Ring and the BABAR Detector 42

2.1 Introduction 42

2.2 The PEP-II Storage Ring 43

2.2.1 Beam Parameters 43

2.2.2 Interaction Point 44

2.2.3 Machine Backgrounds 44

2.2.4 Performance 46

2.3 The BABAR Detector $\quad 46$

2.4 The Silicon Vertex Tracker (SVT) 47

$\begin{array}{lll}2.4 .1 & \text { Requirements } & 47\end{array}$

2.4.2 Design 48

2.4.3 Performance 50

2.5 The Drift Chamber $(\mathrm{DCH}) \quad 52$

2.5.1 Requirements $\quad 52$

2.5.2 Design $\quad 52$

2.5.3 Performance 54

2.5.4 Combined SVT and DCH Tracking Performance 55

2.6 The Detector of Internally Reflected Cherenkov Light (DIRC) 57

$\begin{array}{lll}2.6 .1 & \text { Requirements } & 57\end{array}$

$\begin{array}{lll}2.6 .2 & \text { Design } & 58\end{array}$

$\begin{array}{lll}2.6 .3 & \text { Performance } & 60\end{array}$

2.7 The Electromagnetic Calorimeter (EMC) 61

$\begin{array}{lll}2.7 .1 & \text { Requirements } & 61\end{array}$

$\begin{array}{lll}2.7 .2 & \text { Design } & 61\end{array}$

2.7.3 Performance 64

2.8 The Instrumented Flux Return (IFR) 66

$\begin{array}{lll}2.8 .1 & \text { Requirements } & 66\end{array}$

$\begin{array}{lll}2.8 .2 & \text { Design } & 67\end{array}$

$\begin{array}{lll}2.8 .3 & \text { Performance } & 69\end{array}$

2.9 The Trigger 70

$\begin{array}{lll}2.9 .1 & \text { Requirements } & 70\end{array}$

$\begin{array}{lll}2.9 .2 & \text { Design } & 71\end{array}$

$\begin{array}{lll}2.9 .3 & \text { Performance } & 72\end{array}$ 
$\begin{array}{lll}\text { Chapter 3. } & \text { EMC Calibrations } & 74\end{array}$

$\begin{array}{lll}3.1 & \text { Introduction } & 74\end{array}$

3.2 The EMC Electronics

$\begin{array}{lll}3.3 & \text { Electronics Calibration } & 76\end{array}$

3.4 Non-Linearities in the EMC Electronics Response. $\quad 77$

$\begin{array}{lll}3.4 .1 & \text { ADB Oscillation } & 78\end{array}$

$\begin{array}{lll}3.4 .2 & \text { Crosstalk } & 79\end{array}$

3.5 Remaining Non-Linearities at Range Switches 80

3.5.1 Structure in Crates 84

3.5.2 Crosstalk Corrections 84

3.6 Non-Linearities Correction $\quad 85$

3.7 Corrected Digi Spectra 86

$\begin{array}{lll}3.8 & \text { Conclusions } & 87\end{array}$

Chapter 4. Event Selection and Neutrino Reconstruction 90

4.1 Introduction 90

4.2 Data Set and Monte Carlo Samples 90

4.3 Event Preselection 93

4.4 Track Selection 93

$\begin{array}{lll}4.5 & \text { Neutral Selection } & 95\end{array}$

$\begin{array}{lll}\text { 4.5.1 Photons } & 95\end{array}$

4.5.2 $\pi^{0}$ Reconstruction 96

$\begin{array}{lll}4.6 & \text { Lepton Selection } & 96\end{array}$

4.6.1 Electron Identification 96

4.6.2 Muon Identification 97

$\begin{array}{lll}\text { 4.6.3 Fake Leptons } & 98\end{array}$

4.7 Hadron Selection 99

$\begin{array}{lll}4.7 .1 & B \rightarrow \pi l \nu & 99\end{array}$

$\begin{array}{lll}4.7 .2 \quad B \rightarrow \rho l \nu & 100\end{array}$

$\begin{array}{lll}\text { 4.7.3 } B \rightarrow \omega l \nu & 100\end{array}$

4.8 Neutrino Reconstruction 101

$\begin{array}{lll}4.9 & \text { Selection Criteria } & 102\end{array}$ 
4.9.1 Event Shape Variables 102

$\begin{array}{lll}4.9 .2 & \text { Kinematic Selection } & 103\end{array}$

$\begin{array}{lll}\text { 4.9.3 Neutrino Selection } & 105\end{array}$

4.10 Fit Variables 106

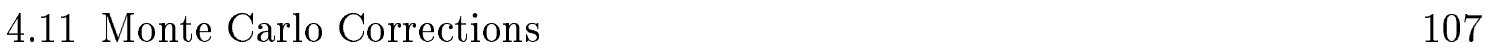

4.11.1 Charged PID Efficiency Correction 108

4.11.2 Neutrals Efficiency and Resolution Corrections 108

$\begin{array}{ll}\text { 4.11.3 Tracking Efficiency Correction } & 108\end{array}$

4.12 Data/MC Comparisons 109

4.12.1 Selection of $B^{0} \rightarrow D^{*-} \ell^{+} \nu$ Decays 109

4.12.2 Resolution in Missing Momentum and Energy 110

4.12.3 Efficiency Studies 111

4.13 Efficiencies 116

Chapter 5. Maximum Likelihood Fit and Systematics $\quad 126$

$\begin{array}{llr}5.1 & \text { Introduction } & 126\end{array}$

5.2 The Fit 126

$\begin{array}{lll}\text { 5.2.1 Fit Method } & 127\end{array}$

$\begin{array}{lll}\text { 5.2.2 } & \text { Fit Contributions } & 129\end{array}$

$\begin{array}{lll}\text { 5.2.3 Fit Binning } & 132\end{array}$

$\begin{array}{lll}5.2 .4 & \text { Fit Validation } & 133\end{array}$

$\begin{array}{lll}5.3 & \text { Results } & 135\end{array}$

5.3.1 Branching Fraction Fit Results 135

$\begin{array}{lll}5.3 .2 & \text { Fit Projections } & 135\end{array}$

$\begin{array}{lll}5.3 .3 & \text { Fit Quality } & 139\end{array}$

5.3.4 Control Sample Branching Fractions 140

5.4 Systematics and Checks $\quad 140$

5.4.1 Lepton PID 140

5.4.2 Fake Lepton Background 141

5.4.3 Tracking Efficiency 141

5.4.4 Neutrals Correction 142

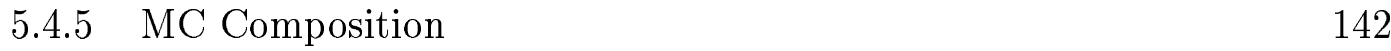


5.4.6 Fitting Systematics 144

$\begin{array}{lll}5.4 .7 & \text { Isospin Violation } & 145\end{array}$

5.4.8 Non- $B$ Background Scale 146

$\begin{array}{lll}5.4 .9 & B \text { Counting } & 147\end{array}$

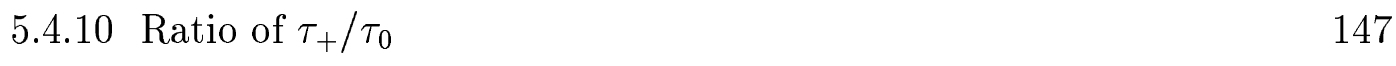

5.4.11 Missing Mass Efficiency Corrections 147

5.4.12 Combined Systematic Error 147

$\begin{array}{lll}5.5 & \text { Extraction of }\left|V_{u b}\right| & 148\end{array}$

$\begin{array}{ll}\text { Chapter 6. Conclusions } & 149\end{array}$

$\begin{array}{lll}6.1 & \text { Introduction } & 149\end{array}$

6.2 EMC Non-linearities 149

$\begin{array}{lll}6.3 & \text { Branching Fractions } & 150\end{array}$

$\begin{array}{lll}6.4\left|\mathbf{V}_{\mathbf{u b}}\right| & 150\end{array}$

6.5 Future Work 150

$\begin{array}{ll}\text { References } & 152\end{array}$ 


\section{List of Figures}

1.1 The unitarity triangle and its renormalised version

1.2 The tree (left) and penguin (right) Feynman diagram for the decay $B^{0} \rightarrow$ $J / \psi K_{S}^{0}$.

1.3 The plot shows the $(\bar{\rho}, \bar{\eta})$ plane with the current constraints on the CKM matrix. The unitarity triangle is superimposed on the plot.

1.4 Feynman diagram for charmless semileptonic decay of a $B$ meson.

1.5 The definition of the angles that occur in the semileptonic decay of a $B$ meson to a vector meson, which subsequently decays to two pseudoscalar mesons.

2.1 A schematic of the PEP-II elements at the BABAR IP.

2.2 Luminosity delivered by PEP-II and recorded by BABAR.

2.3 Cross section of the BABAR detector.

2.4 Schematic of SVT silicon layers in the $\mathrm{x}-\mathrm{y}$ plane.

2.5 Schematic of SVT silicon layers in the $\mathrm{z}$ plane.

2.6 The resolution of hits in the SVT for the a) $\mathrm{z}$ and b) $\phi$ direction as a function of incidence angle. Each plot shows a different layer.

2.7 Cross section of the DCH.

2.8 First four superlayers of the DCH.

$2.9 \mathrm{~d} E / \mathrm{d} x$ of particles measured in the $\mathrm{DCH}$. The solid lines show the BetheBloch predictions derived from control samples. The y axis is in arbitrary units. 
2.10 The plots shows the ratio of tracks reconstructed in the $\mathrm{DCH}$ compared to tracks reconstructed in the SVT (confined to the DCH acceptance). The filled circles are with the DCH operating at 1960V, the open circles at $1900 \mathrm{~V}$.

2.11 Resolution of track parameters measured by taking the difference of the two halves of the tracks fitted to cosmic ray muons. The tracks have $p_{T}>3 \mathrm{GeV} / c$.

2.12 Resolution of $p_{T}$ from tracks with both DCH and SVT hits, measured using cosmic ray data.

2.13 Cross section of the DIRC.

$2.14 e^{+} e^{-} \rightarrow \mu^{+} \mu^{-}$event reconstructed using two different time cuts. The left shows hits within $\pm 300 \mathrm{~ns}$, on the right the time window has been reduced to \pm 8 ns of the expected arrival time.

2.15 The difference between (a) the measured and expected Cherenkov angle for single photons $\Delta \theta_{c, \gamma}$ and (b) the measured and expected photon arrival time for $\mu^{+} \mu^{-}$events.

2.16 The difference between measured and expected Cherenkov angle $\left(\Delta \Theta_{c, \text { track }}\right)$ for $\mu^{+} \mu^{-}$events.

2.17 Cross section of the top half of the EMC.

2.18 The energy resolution of the EMC measured using various processes. The inner solid line represents a fit to equation 2.3 , the two outer lines denote the RMS error of the fit.

2.19 The angular resolution of the EMC measured photons from $\pi^{0}$ decays. The solid line is a fit to equation 2.4

2.20 The invariant mass of two photon combinations in $B \bar{B}$ decays.

2.21 The electron identification efficiency (shown on the left scale) and the pion misidentification probability (right scale) as a function of momentum and polar angle.

2.22 Schematic of the IFR.

2.23 Cross section of an RPC module.

2.24 Muon identification efficiency (left scale) and pion misidentification probability (right scale) as a function of momentum and polar angle. 
2.25 The distance of closest approach of single L1 tracks to the IP in the $\mathrm{Z}$ direction as reconstructed by L3.

3.1 The EMC readout electronics chain.

3.2 The energy spectrum of data of all channels (blue curve) for the first year of running (Run1). The $\times 256-\times 32$ range switch occurs at $\approx-1.4$ which corresponds to an energy of $50 \mathrm{MeV}$. The $\times 32-\times 4$ switch is at $-0.45(400 \mathrm{MeV})$ and the $\times 4-\times 1$ at $0.4(3.2 \mathrm{GeV})$. The overlayed green curve shows the resultant energy spectrum after an offline correction has been applied [1].

3.3 The energy spectrum (blue curve) of all channels for Run2 (2002) data. The overlayed green curve shows the resultant energy spectrum after an offline correction has been applied [1].

3.4 The plot shows the LUT count against DAC response for the same channel using two different modes of data taking. The red (+) points show data taken in auto-ranging mode and the black $(\times)$ using the $\times 32$ amplifier. A deviation between the two sets of data is apparent at the point where the auto-ranging mode switches between the $\times 256$ amplifier and the $\times 32$ amplifier at around 1110 DAC counts.

3.5 The plots show the average percentage deviation between the auto-ranging mode of operation and the fixed amplifier mode across the amplifier switch for all three gain ranges and for all channels in one crate. The topmost set of plots correspond to crate 7, which is situated in the forward barrel and has high gain preamplifiers. The middle set of plots show crate 3 data, a forward barrel crate with low gain preamplifiers. The lower plots correspond to crate 8 in the end cap.

3.6 The left plot shows the maximum deviation per channel averaged over mini crates in crate 7 for the $\times 1-\times 4$ range switch. The right plot shows the average deviation separated into ADBs (12 channels). Higher channels are further into the barrel than lower channels. 
3.7 Each plot shows the average deviation between fixed and auto ranging operation for odd (black) and even (red) channels. For the plot in the left the crosstalk corection has been applied. There was no cross talk correction for the plot on the right

3.8 The plots show the average percentage deviation for each of the 3 gain range switches for crate 7 before (black) and after the correction (red).

3.9 The plots show single crystal energy spectra for data taken during Run2. The upper set of 12 plots (which corresponds to 1 ADB) show the energy spectra before the range change correction is applied. The lower plots show the same channels after the correction.

3.10 The plots show the energy spectra taken from Bhabha events separated into theta rings. The $\mathrm{x}$-axis units are $\log _{10}[\mathrm{GeV}]$. The blue plots were taken before the auto-range correction was implemented and the red plots were after the implementation.

4.1 The Dalitz Amplitude of $B^{+} \rightarrow \omega l^{+} \nu$ mode. The true $\mathrm{MC} B^{+} \rightarrow \omega l^{+} \nu$ decays are shown in black and the combinatoric generic $B \bar{B}$ background decays in dashed red.

4.2 Kinematics of $B \rightarrow X_{u} l \nu$ decays. $Y$ represents the combined $X_{u} l$ system. 104

4.3 Distribution of $\cos \theta_{B Y}$. The left plot shows the distribution of $\cos \theta_{B Y}$ decays reconstructed in the $B^{0} \rightarrow \pi^{-} l^{+} \nu$ mode from Monte Carlo samples. The correctly reconstructed signal decays are shown in black, generic $B^{0}$ decays in green and off-resonance in red. The right plot shows the $\cos \theta_{B Y}$ distributions for correctly reconstructed signal decays separated into electrons (solid) and muons (dashed).

$4.4|\Delta \theta|$ for events reconstructed in the $B^{0} \rightarrow \pi^{-} \ell^{+} \nu$ mode after all other selection has been applied. The true $\mathrm{MC} B^{0} \rightarrow \pi^{-} \ell^{+} \nu$ decays are shown in black and generic $B \bar{B}$ background decays in dashed red.

4.5 MC predicted resolutions of the momentum magnitude (left), energy (right), direction (right) of the missing particle for the $\overline{D^{0}} \rightarrow K^{+} \pi^{-}$ subsample of the control sample. 
4.6 The plots show the comparison between data and Monte Carlo simulation, normalised to the same area, and the ratio of the two in each bin (smaller plots). The upper set of plots show distributions before the main event selection (but after $D^{*}$ selection). All event selection (apart from on the quantity plotted) has been applied to the lower set of plots. The first column shows the $M_{M i s s}^{2}$, the second shows the $M_{m i s s}^{2} / 2 E_{m i s s}$, the total charge of the event is shown in the third column and the lepton momentum spectra in the last column. The contributions to each histogram, from top to bottom are, fake lepton (blue), continuum background (green), fake $D *$ (as determined from Monte Carlo truth, red), true $D^{*}$ (black) and true signal decays (blank). These data are the $\overline{D^{0}} \rightarrow K \pi$ control sample.

4.7 The plots show the comparison between data and Monte Carlo simulation, normalised to the same area, and the ratio of the two in each bin (smaller plots). The upper plots show distributions before the main event selection (but after $\mathrm{D}^{*}$ selection), all event selection has been applied to the lower plots. The first column shows the number of tracks in the event (excluding the signal tracks) and the second the momenta of the tracks. The number of clusters per event is shown in the third column and the energy of the clusters in the last. The histogram contributions are the same as defined in 4.6. All plots correspond to the $\overline{D^{0}} \rightarrow K^{+} \pi^{-}$control sample

4.8 The plots show the comparison between data and Monte Carlo simulation, normalised to the same area, and the ratio of the two in each bin (smaller plots). The upper plots show distributions before the main event selection (but after $\mathrm{D}^{*}$ selection), all event selection apart from $p_{\text {miss }}$ vs $\theta_{\text {miss }}$ has been applied to the lower plots in the first three columns. All selection has been applied to the lower plot in the final column. The first column shows the reconstructed missing momentum while the missing energy is shown in the second. The direction of the reconstructed neutrino (third set of plots) and $Q^{2}$ is also plotted. The histogram contributions are the same as defined in 4.6. All plots correspond to the $\overline{D^{0}} \rightarrow K^{+} \pi^{-}$control sample 
4.9 The plots show the comparison between data and Monte Carlo simulation, normalised to the same area, and the ratio of the two in each bin (smaller plots). Signal region and side band projections of $m_{E S}$ and $\Delta E$ are shown. The upper plots show distributions before the main event selection (but after $\mathrm{D}^{*}$ selection), all event selection has been applied to the lower plots. The histogram contributions are the same as defined in 4.6. All plots correspond to the $\overline{D^{0}} \rightarrow K^{+} \pi^{-}$control sample

5.1 Projections of the fit to the off-resonanc data for the $B^{0} \rightarrow \pi^{-} l^{+} \nu$ reconstruction mode. The lower plot in each set shows the ratio of the data and the fit projection. The green histogram is the fake lepton contribution and the red the non- $B$ background from Monte Carlo. The plots on the left are for electrons and the right muons. The fake lepton background dominates for the muon.

5.2 The distribution of the $B \rightarrow \pi^{-} l^{+} \nu$ signal events across the fit plane. The grid shows the choice of binning used in the fit.

5.3 The pull distributions for a set of 1000 toy Monte Carlo experiments. The top left plot shows the pull for $\mathcal{B}\left(B \rightarrow \pi^{ \pm} l \nu\right)$, the top right the pull for $\mathcal{B}\left(B \rightarrow \rho^{ \pm} l \nu\right)$ and the bottom plot $\mathcal{B}(B \rightarrow \omega l \nu)$. The distributions are fitted with a Gaussian function

5.4 $\Delta E$ and $m_{E S}$ projections for $B^{0} \rightarrow \pi^{-} \ell^{+} \nu$. The left plots show the projections for $\Delta E$ in the $m_{E S}$ signal (top set of plots) and $m_{E S}$ sideband (bottom plots) regions. On the right are the corresponding plots for $m_{E S}$. The lower plot in each set shows the ratio of the data and the fit results in each bin. The histogram components, from bottom to top, are fake leptons (black), continuum (red), secondary leptons (yellow dashed), $b \rightarrow c l \nu$ decays (yellow), $b \rightarrow$ ulv feeddown (vertical lines), vector mode cross-feed (cross hatched), pion cross-feed (open dashed histogram, this includes incorrectly reconstructed signal decays) and true signal decays (open).

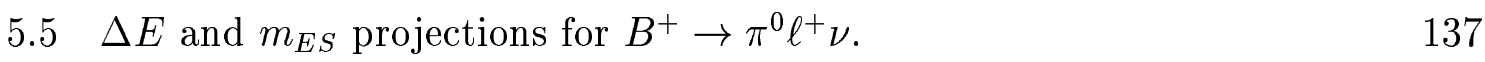

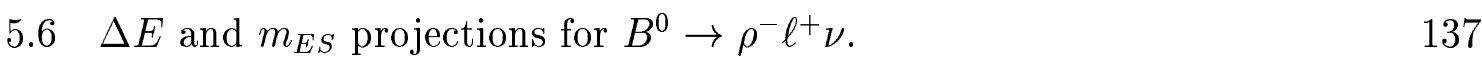


5.7 $\Delta E$ and $m_{E S}$ projections for $B^{+} \rightarrow \rho^{0} \ell^{+} \nu . \quad 138$

$5.8 \Delta E$ and $m_{E S}$ projections for $B^{+} \rightarrow \omega^{0} \ell^{+} \nu$. 138

5.9 The values of $\ln \mathcal{L}_{\text {max }}$ determined from a set of 1000 toy Monte Carlo experiments generated using the parameters determined by the fit to data. The arrow shows the value of $\ln \mathcal{L}_{\max }$ for the fit to data 


\section{List of Tables}

1.1 Summary of $\left|V_{u b}\right|$ exclusive measurements by experiment. The table gives the result along with the decay mode studied and the method used (tight $\nu$ indicates that tight neutrino reconstruction has been employed). For the first two results the errors are statistical, experimental systematic and form factor modelling. The next two CLEO results give statistical, experimental systematic, $\rho l \nu$ form factor shape, and form factor normalisation errors. The first two Belle results quote statistical, experimental systematic and form factor model uncertainties, while the final result just gives statistical and experimental systematic errors.

1.2 Summary of $\left|V_{u b}\right|$ inclusive measurements by experiment. The result, with experimental and theoretical errors are given, along with the method used.

2.1 Parameters of the PEP-II machine. The typical values are the average values achieved during the first year of running. The third column gives the best values achieved to date

2.2 L1 and L3 combined efficiency for various physics processes, derived from Monte Carlo simulations.

4.1 Production cross-sections at the $\Upsilon(4 S)$ resonance. The $e^{+} e^{-}$cross-section given is the effective cross-section expected within the experimental acceptance.

4.2 The table shows the $B \rightarrow X_{u} l \nu$ branching fractions (excluding signal decays) used to generate the generic $B$ Monte Carlo. The inclusive fraction is purely non-resonant pion decays. 
4.3 The table shows the $B \rightarrow X_{c} l \nu$ branching fractions. $B^{0} \rightarrow c l \nu$ includes all charmed modes other than those given

4.4 Additional criteria used to remove duplicate tracks.

4.5 Comparison of the number of events and the resulting efficiencies for the electron mode of the $\overline{D^{0}} \rightarrow K^{+} \pi^{-}$control sample for data and MC events. The efficiencies are evaluated sequentially, i.e. with previous cuts applied. The numbers are determined by counting the number of events in the $\Delta E, m_{E S}$ fit plane.

4.6 Comparison of the number of events and the resulting efficiencies for the muon mode of the $\overline{D^{0}} \rightarrow K^{+} \pi^{-}$control sample for data and MC events. The efficiencies are evaluated sequentially, i.e. with previous cuts applied. The numbers are determined by counting the number of events in the $\Delta E, m_{E S}$ fit plane.

4.7 Comparison of the number of events and the resulting efficiencies for the electron mode of the $\overline{D^{0}} \rightarrow K^{+} \pi^{-} \pi^{0}$ control sample for data and MC. The efficiencies are evaluated sequentially, i.e. with previous cuts applied. The numbers are determined by counting the number of events in the $\Delta E, m_{E S}$ fit plane.

4.8 Comparison of the number of events and the resulting efficiencies for the muon mode of the $\overline{D^{0}} \rightarrow K^{+} \pi^{-} \pi^{0}$ control sample for data and MC. The efficiencies are evaluated sequentially, i.e. with previous cuts applied. The numbers are determined by counting the number of events in the $\Delta E, m_{E S}$ fit plane.

4.9 Comparison of the number of events and the resulting efficiencies for the electron mode of the $\overline{D^{0}} \rightarrow K^{+} \pi^{-} \pi^{+} \pi^{-}$control sample for data and MC. The efficiencies are evaluated sequentially, i.e. with previous cuts applied. The numbers are determined by counting the number of events in the $\Delta E, m_{E S}$ fit plane.

4.10 Comparison of the number of events and the resulting efficiencies for the muon mode of the $\overline{D^{0}} \rightarrow K^{+} \pi^{-} \pi^{+} \pi^{-}$control sample for data and MC. The efficiencies are evaluated sequentially, i.e. with previous cuts applied. The numbers are determined by counting the number of events in the $\Delta E, m_{E S}$ fit plane. 
4.11 Final selection efficiencies for the five signal modes. The "signal" column gives the efficiencies for correctly reconstructed signal decays according to Monte Carlo truth. The other columns give the crossfeed between the reconstruction modes. These efficiencies combine electron and muon decays.

4.12 Efficiencies for the $B^{0} \rightarrow \pi^{-} l^{+} \nu$ mode. The "sig" column is the efficiencies for correctly reconstructed signal decays (according to Monte Carlo truth information). The $B \rightarrow c l \nu$ column show the efficiencies for the semileptonic charm background, the $B \rightarrow$ other background from secondary leptons, the $b \bar{b}$ is background from hadrons faking leptons in $B$ meson decays, $q \bar{q}$ is continuum background and $q \bar{q}$ fake is from hadrons faking leptons in continuum decays. The efficiency for each cut is the incremental efficiency of that cut, i.e. applying the cut after all other cuts are applied.

4.13 Efficiencies for the $B^{+} \rightarrow \pi^{0} l^{+} \nu$ mode. The "sig" column is the efficiencies for correctly reconstructed signal decays (according to Monte Carlo truth information). The $B \rightarrow c l \nu$ column show the efficiencies for the semileptonic charm background, the $B \rightarrow$ other background from secondary leptons, the $b \bar{b}$ is background from hadrons faking leptons in $B$ meson decays, $q \bar{q}$ is continuum background and $q \bar{q}$ fake is from hadrons faking leptons in continuum decays. The efficiency for each cut is the incremental efficiency of that cut, i.e. applying the cut after all other cuts are applied.

4.14 Efficiencies for the $B^{0} \rightarrow \rho^{-} l^{+} \nu$ mode. The "sig" column is the efficiencies for correctly reconstructed signal decays (according to Monte Carlo truth information). The $B \rightarrow c l \nu$ column show the efficiencies for the semileptonic charm background, the $B \rightarrow$ other background from secondary leptons, the $b \bar{b}$ is background from hadrons faking leptons in $B$ meson decays, $q \bar{q}$ is continuum background and $q \bar{q}$ fake is from hadrons faking leptons in continuum decays. The efficiency for each cut is the incremental efficiency of that cut, i.e. applying the cut after all other cuts are applied. 
4.15 Efficiencies for the $B^{+} \rightarrow \rho^{0} l^{+} \nu$ mode. The "sig" column is the efficiencies for correctly reconstructed signal decays (according to Monte Carlo truth information). The $B \rightarrow c l \nu$ column show the efficiencies for the semileptonic charm background, the $B \rightarrow$ other background from secondary leptons, the $b \bar{b}$ is background from hadrons faking leptons in $B$ meson decays, $q \bar{q}$ is continuum background and $q \bar{q}$ fake is from hadrons faking leptons in continuum decays. The efficiency for each cut is the incremental efficiency of that cut, i.e. applying the cut after all other cuts are applied.

4.16 Efficiencies for the $B^{+} \rightarrow \omega l^{+} \nu$ mode. The "sig" column is the efficiencies for correctly reconstructed signal decays (according to Monte Carlo truth information). The $B \rightarrow c l \nu$ column show the efficiencies for the semileptonic charm background, the $B \rightarrow$ other background from secondary leptons, the $b \bar{b}$ is background from hadrons faking leptons in $B$ meson decays, $q \bar{q}$ is continuum background and $q \bar{q}$ fake is from hadrons faking leptons in continuum decays. The efficiency for each cut is the incremental efficiency of that cut, i.e. applying the cut after all other cuts are applied.

5.1 The table shows the parameters used in the fit. The last coloumn shows which parameters are free in the fit and which are fixed.

5.2 The branching fractions and scale factors determined by the fit.

5.3 The correlations between fit parameters. The parameters are defined as in the Table 5.1.

$5.4 b \rightarrow c l \nu$ (upper) and $b \rightarrow u l \nu$ (lower) branching fraction variations and the associated systematic error. For the $b \rightarrow c l \nu$ decays the $D^{*}$ and $D$ meson decays are shifted independently and then the remainder of the $B \rightarrow c l \nu$ backgound is shifted. For the $b \rightarrow u l \nu$ background the exclusive $\eta, a, b$ and $f$ meson modes are each varied. The remaining $B \rightarrow u l \nu$ background is modelled as non-resonant decays and also varied by $50 \%$.

5.5 The branching ratios and scale factors determined ignoring Monte Carlo statistics in the fit 
5.6 The impact of different fit binning on the measured branching fractions. 145

5.7 The branching fractions and scale factors determined by the fit for electrons and muon separately.

5.8 The branching ratios and scale factors determined without isospin constraints

5.9 The branching ratios and scale factors determined without isospin constraints, and setting $\eta_{4}(b \rightarrow c \ell \nu+\pi)=\eta_{7}\left(b \rightarrow c \ell \nu+\pi^{0}\right)$ and $\eta_{5}(b \rightarrow$ $c \ell \nu+\rho)=\eta_{8}\left(b \rightarrow c \ell \nu+\rho^{0}\right)$.

5.10 Branching fraction variations and the associated systematic error. 


\section{Chapter 1}

\section{Theory}

\subsection{Introduction}

Our current understanding of particle physics is encapsulated in one of the most successful theories of modern times, the Standard Model. It is successful in the sense that it has been able to predict almost all experimentally tested phenomena so far. It is however far from a perfect theory; it requires the input of many (18) fundamental parameters. One area of the Standard Model which is still poorly tested experimentally, and has the potential to provide indications of "new physics", is the physics of weak decays of heavy mesons. In particular it allows the study of quark mixing, the unitarity of the Cabibbo-Kobayashi-Maskawa (CKM) matrix [2] and the phenomenon of $C P$ violation.

$C P$ violation was observed in $\mathrm{K}$ mesons in 1964 [3]. It is one of the Sakharov conditions needed to explain the baryon asymmetry in the Universe [4]. $C P$ violation is accommodated into the Standard Model with three generations of quarks via a single weak phase in the CKM quark-mixing matrix. However the amount of $C P$ violation predicted by the Standard Model falls short of that required to explain the observed baryon asymmetry.

The mechanism by which the CKM matrix is generated in the Standard Model implies that it must be unitary. Any deviation from unitarity indicates physics 
beyond the Standard Model. By measuring the elements of the matrix we can hope to constrain the CKM matrix.

The unprecedented data set recorded at the new $B$ factories at BELLE and BABAR will considerably enhance our knowledge of $B$ meson decays, allowing us to constrain the CKM matrix and measure $C P$ violation, which should be more readily observable in $B$ mesons than for $K$ mesons.

This chapter reviews the Standard Model prescription for charged weak decays. It will mention the formalism for $C P$ violation and describe the CKM matrix. The current experimental measurements of the CKM matrix elements will be given. The tools necessary in measuring the CKM matrix $\left|V_{u b}\right|$ using semileptonic $B$ decays will be discussed. This includes the form factors which describe the strong aspects of the decay process. A more thorough discussion of $C P$ violation and the CKM matrix can be found in [5-7]. Semileptonic $B$ decays are discussed at length in [8].

\subsection{CP Violation}

The parity transformation $(P)$ is a space-time transform under which $t \rightarrow t, \vec{x} \rightarrow-\vec{x}$. Time reversal $(T)$ sends $t \rightarrow-t$, interchanging the forward and backward light cone. A third discrete operation is charge conjugation $(C)$ that interchanges particles and antiparticles. For pseudoscalar mesons $B$ and $\bar{B}$, using the common phase conventions

$$
\begin{array}{rc}
P|B(\vec{p})\rangle=-|B(-\vec{p})\rangle, & P|\bar{B}(\vec{p})\rangle=-|\bar{B}(-\vec{p})\rangle \\
C|B(\vec{p})\rangle=|\bar{B}(\vec{p})\rangle, & C|\bar{B}(\vec{p})\rangle=|B(\vec{p})\rangle
\end{array}
$$

then the combined $C P$ transform on a pseudoscalar meson is

$$
C P|B(\vec{p})\rangle=-|\bar{B}(-\vec{p})\rangle, \quad C P|\bar{B}(\vec{p})\rangle=-|B(-\vec{p})\rangle
$$

For neutral mesons, $B^{0}$ and $\bar{B}^{0}, C P$ eigenstates can be constructed

$$
\left|B_{1}^{0}\right\rangle=\frac{1}{\sqrt{2}}\left(\left|B^{0}\right\rangle-\left|\bar{B}^{0}\right\rangle\right), \quad\left|B_{2}^{0}\right\rangle=\frac{1}{\sqrt{2}}\left(\left|B^{0}\right\rangle+\left|\bar{B}^{0}\right\rangle\right)
$$


where

$$
C P\left|B_{1}^{0}\right\rangle=\left|B_{1}^{0}\right\rangle, \quad C P\left|B_{2}^{0}\right\rangle=-\left|B_{2}^{0}\right\rangle .
$$

The combination of $C P T$ is an exact symmetry in any causal local Lagrangian field theory.

\subsubsection{Direct CP Violation}

Consider decays of $P$ mesons to final states f:

$$
C P|P\rangle=e^{i \phi_{P}}|\bar{P}\rangle, \quad C P|f\rangle=e^{i \phi_{f}}|\bar{f}\rangle
$$

where $\phi_{P}$ and $\phi_{f}$ are arbitrary phases. The $C P$-conjugated decay amplitudes, $A$ and $\bar{A}$ can be written as

$$
\begin{aligned}
A & =\langle f|H| P\rangle=\sum_{i} A_{i} e^{i \delta_{i}} e^{i \phi_{i}} \\
\bar{A} & =\langle\bar{f}|H| \bar{P}\rangle=e^{i\left(\phi_{P}-\phi_{f}\right)} \sum_{i} A_{i} e^{i \delta_{i}} e^{-i \phi_{i}}
\end{aligned}
$$

where $H$ is the effective Hamiltonian and $A_{i}$ are real partial amplitudes. $\phi_{i}$ are weak phases that violate $C P, \delta_{i}$ are strong phases from scattering amplitudes and enter $A$ and $\bar{A}$ with the same sign. The ratio

$$
\left|\frac{\bar{A}}{A}\right|=\left|\frac{\sum_{i} A_{i} e^{i \delta} e^{-i \phi_{i}}}{\sum_{i} A_{i} e^{i \delta} e^{i \phi_{i}}}\right|
$$

is independent of phase convention and physically meaningful. If $|\bar{A} / A| \neq 1$ then we have direct $C P$ violation. Direct $C P$ violation requires at least two partial amplitudes that differ in both strong and weak phases.

\subsubsection{Indirect CP Violation}

If neutral mesons are able to mix through $B^{0} \rightarrow \bar{B}^{0}$, then the physical states can be written as a superposition of the flavour eigenstates, $a|B\rangle+b|\bar{B}\rangle$, which obeys the 
Schrödinger equation

$$
i \frac{d}{d t}\left(\begin{array}{c}
a \\
b
\end{array}\right)=\mathbf{H}\left(\begin{array}{c}
a \\
b
\end{array}\right)=\left(\mathbf{M}-\frac{i}{2} \Gamma\right)\left(\begin{array}{c}
a \\
b
\end{array}\right)
$$

where $\mathbf{H}$ and $\boldsymbol{\Gamma}$ are Hermitian $2 \times 2$ matrices. The physical eigenstates are given in terms of the eigenvectors

$$
\left|B_{1,2}\right\rangle=p\left|B^{0}\right\rangle \pm q\left|\bar{B}^{0}\right\rangle ; \quad|p|^{2}+|q|^{2}=1
$$

The ratio

$$
\left|\frac{q}{p}\right|^{2}=\left|\frac{M_{12}^{*}-\frac{i}{2} \Gamma_{12}^{*}}{M_{12}-\frac{i}{2} \Gamma_{12}}\right|
$$

is independent of phase convention and therefore physically meaningful. $|q / p| \neq 1$ implies indirect $C P$ violation.

\subsubsection{CP Violation in the Interference of Mixing and Decay}

This occurs when neutral mesons decay to $C P$ eigenstates:

$$
A=\left\langle f_{C P}|H| B^{0}\right\rangle, \quad \bar{A}=\left\langle f_{C P}|H| \bar{B}^{0}\right\rangle
$$

In this case the product

$$
\lambda=\frac{q}{p} \cdot \frac{\bar{A}}{A}
$$

is independent of phase convention and physically meaningful. The condition $\lambda \neq$ \pm 1 implies $C P$ violation. Note that direct $C P$ violation $(|\bar{A} / A| \neq 1)$ and indirect $C P$ violation $(|q / p| \neq 1)$ imply $|\lambda| \neq 1$, but it is possible to have no direct or indirect $C P$ violation and still have $\lambda \neq \pm 1$,

$$
|\lambda|=1, \quad \operatorname{Im}(\lambda) \neq 0
$$

\subsection{The Standard Electroweak Model}

The standard electroweak model or Glashow-Weinberg-Salam model is based on the gauge group $\mathrm{SU}(2) \times \mathrm{U}(1)$ [9-11]. In the Standard Model there are three generations 
of leptons $\left(\begin{array}{c}\nu_{i} \\ l_{i}^{-}\end{array}\right)$and three generations of quarks $\left(\begin{array}{c}u_{i} \\ d_{i}\end{array}\right)$ (where $i$ is the fermion generation). The left handed components of these fermion fields $\chi_{L i}=\left(\begin{array}{c}u_{i} \\ d_{i}\end{array}\right)_{L}$ transform as doublets under weak isospin $\mathrm{SU}(2)$. The right handed fields $q_{R} \stackrel{L}{=}$ $u_{R}\left(d_{R}\right)$ are weak isospin singlets. The $\mathrm{SU}(2)$ group has a gauge coupling constant $g$ and the corresponding gauge bosons are denoted $W_{\mu}^{i}$. $B_{\mu}$ is the gauge boson of the weak hyper-charge $\mathrm{U}(1)$ group, which has a gauge coupling constant $g^{\prime}$. Before spontaneous symmetry breaking the weak Lagrangian for the fermion fields is given by

$$
\begin{aligned}
\mathcal{L} & =\bar{\chi}_{L} \gamma^{\mu}\left(i \partial_{\mu}-\frac{g}{2} \mathbf{T} \cdot \mathbf{W}_{\mu}-\frac{g^{\prime}}{2} Y_{L} \mathbb{1} B_{\mu}\right) \chi_{L} \\
& +\bar{q}_{R} \gamma^{\mu}\left(i \partial_{\mu}-\frac{g^{\prime}}{2} Y_{R} B_{\mu}\right) q_{R}
\end{aligned}
$$

where the operators $\mathbf{T}$ and $Y$ are the generators of the $\mathrm{SU}(2)$ and $\mathrm{U}(1)$ groups respectively. The two neutral physical gauge fields $Z_{\mu}^{0}$ and $A_{\mu}$ (the photon field) are orthogonal superpositions of $B_{\mu}$ and $W_{\mu}^{3}$,

$$
\begin{aligned}
A_{\mu} & =B_{\mu} \cos \left(\theta_{W}\right)+W_{\mu}^{3} \sin \left(\theta_{W}\right), \\
Z_{\mu} & =-B_{\mu} \sin \left(\theta_{W}\right)+W_{\mu}^{3} \cos \left(\theta_{W}\right),
\end{aligned}
$$

where $\theta_{W}$ is the weak mixing angle.

As yet this Lagrangian only describes massless fermions and gauge bosons. Masses are incorporated by spontaneous symmetry breaking using the Higgs mechanism. A scalar doublet $\phi=\left(\begin{array}{c}\phi^{-} \\ \phi^{0}\end{array}\right)$ is introduced which is invariant under $\mathrm{SU}(2) \times \mathrm{U}(1)$. The symmetry is then broken by choosing a minimum in the Higgs potential. The gauge bosons $W_{\mu}^{+}, W_{\mu}^{-}$and $Z_{\mu}^{0}$ become massive particles, but the photon field $A_{\mu}$ remains massless. The fermions acquire masses through Yukawa type couplings of the form

$$
-G_{j}^{d}\left(\bar{\chi}_{L j} \phi\right) d_{R j}-G_{j}^{U}\left(\bar{\chi}_{L j} \tilde{\phi}\right) u_{R j}+\text { h.c. }
$$

For more than one generation of quarks it is possible to have Yukawa terms that mix the quarks. After setting the Higgs potential to the vacuum expectation value, $\phi=\left(\begin{array}{c}0 \\ v+h\end{array}\right)$ the mass terms become

$$
\frac{v}{\sqrt{2}}\left(D_{i j} \bar{d}_{L i} d_{R j}+U_{i j} \bar{u}_{L i} u_{R j}\right)+\text { h.c. }
$$


where, for three generations of quarks, $\mathbf{D}$ and $\mathbf{U}$ are arbitrary $3 \times 3$ matrices. The mass states of the quarks are found by diagonalising $\mathbf{D}$ and $\mathbf{U}$ using four unitary matrices

$$
\mathbf{D}_{\text {diag }}=\mathbf{V}_{L}^{d} \mathbf{D V}_{R}^{d \dagger}, \quad \mathbf{U}_{\text {diag }}=\mathbf{V}_{L}^{u} \mathbf{U V}_{R}^{u \dagger}
$$

The physical mass eigenstates of the quarks are then

$$
\begin{aligned}
u_{L i}=V_{L i j}^{u} u_{L j}^{0}, & u_{R i}=V_{R i j}^{u} u_{R j}^{0}, \\
d_{L i}=V_{L i j}^{d} d_{L j}^{0}, & d_{R i}=V_{R i j}^{d} d_{R j}^{0},
\end{aligned}
$$

here $q^{0}$ are the weak eigenstates. Substituting these mass eigenstates into the interaction term for the charged weak current gives

$$
\mathcal{L}_{\text {int }}=-\frac{g}{\sqrt{2}} \bar{u}_{L i} \gamma^{\mu} V_{L i k}^{u} V_{L k j}^{d \dagger} d_{L j} W^{+}+\text {h.c. }
$$

The quark-mixing matrix $\mathbf{V}_{C K M}=\mathbf{V}_{L}^{u} \mathbf{V}_{L}^{d \dagger}$ is the Cabibbo-Kobayashi-Maskawa (CKM) mixing matrix

$$
\mathbf{V}_{C K M}=\left(\begin{array}{ccc}
V_{u d} & V_{u s} & V_{u b} \\
V_{c d} & V_{c s} & V_{c b} \\
V_{t d} & V_{t s} & V_{t b}
\end{array}\right)
$$

\subsection{The CKM Matrix}

The quark-mixing matrix is required to be unitary. A general $3 \times 3$ unitary matrix can be expressed in terms of three real parameters and six phases. In the case of $\mathbf{V}_{C K M}$ five of these phases can be removed by appropriately redefining the relative phases of the six quark fields, which has no effect on physical observables

$$
q_{L i} \rightarrow\left(P_{q}\right)_{i i} q_{L i}, \quad q_{R i} \rightarrow\left(P_{q}\right)_{i i} q_{R i} .
$$

Hence, $\mathbf{V}_{C K M}$ can be expressed in terms of three real angles and one observable phase, as was first noted by Kobayashi and Maskawa [2]. The remaining observable phase allows $C P$ violation to be incorporated into the Standard Model. Note that in general a $n \times n$ quark mixing matrix has $\frac{1}{2} n(n-1)$ angles and $\frac{1}{2}(n-1)(n-2)$ 
observable phases. For the case of 2 generations of quarks the mixing matrix depends upon only one angle, the Cabibbo angle [12], and doesn't allow for $C P$ violation.

$C P$ violation is still not guaranteed by the Standard Model, as a number of conditions must first be met. If two quarks of the same charge have equal mass the observable phase and one angle can be removed. This condition for $C P$ violation can be summarised as

$$
\left(m_{u}^{2}-m_{c}^{2}\right)\left(m_{u}^{2}-m_{t}^{2}\right)\left(m_{c}^{2}-m_{t}^{2}\right)\left(m_{d}^{2}-m_{s}^{2}\right)\left(m_{d}^{2}-m_{b}^{2}\right)\left(m_{s}^{2}-m_{b}^{2}\right) \neq 0
$$

In addition the observable phase can be removed if any of the three angles are equal to 0 or $\pi / 2$, and would also disappear if the phase itself is equal to 0 or $\pi$. These last conditions can be summarised by $J_{C P} \neq 0$ for $C P$ violation, where for any choice of $i, j, k=1,2,3$

$$
J_{C P} \sum \epsilon_{i k m} \epsilon_{j l n}=\operatorname{Im}\left(V_{i j} V_{k l} V_{i l}^{*} V_{k j}^{*}\right),
$$

where $\epsilon_{i k m}$ is the totally antisymmetric tensor. This quantity is invariant under phase redefinitions of the quark fields. All $C P$ violation in the Standard Model is proportional to $J_{C P}$.

The standard parameterisation of $\mathbf{V}_{C K M}$ recommended by the Particle Physics Data group [7] is

$$
\mathbf{V}_{C K M}=\left(\begin{array}{ccc}
c_{12} c_{13} & s_{12} c_{13} & s_{13} e^{-i \delta} \\
-s_{12} c_{23}-c_{12} s_{23} s_{13} e^{-i \delta} & c_{12} c_{23}-s_{12} s_{23} s_{13} e^{-i \delta} & s_{23} c_{13} \\
s_{12} c_{23}-c_{12} c_{23} s_{13} e^{-i \delta} & -c_{12} s_{23}-s_{12} c_{23} s_{13} e^{-i \delta} & c_{23} c_{13}
\end{array}\right)
$$

Here, $c_{i j}=\cos \theta_{i j}$ and $s_{i j}=\sin \theta_{i j}$. This parameterisation has the interpretational advantage that the angles are defined in such a way that they relate to the specific mixing between generations. For example if $\theta_{13}=\theta_{23}=0$ then the third generation decouples and we are left with the usual Cabibbo mixing with $\theta_{12}$ identified as the Cabibbo angle. Another nice feature is that $\left|V_{u b}\right|=s_{13}$ is given by a single angle, which turns out to be small experimentally. This leads to the indication that $C P$ violation should be small in the Standard Model, as the $C P$ violating phase only appears together with $s_{13}$. In this representation $J_{C P}=s_{13} s_{23} s_{12} c_{13}^{2} c_{23} c_{12} \sin \delta$. 
It is sometimes more instructive to represent $\mathbf{V}_{C K M}$ in a manner which emphasises the hierarchy of the mixing angle. Experimentally $c_{13}$ is known to deviate from unity only in the sixth decimal place, so can be approximated to 1 . We now denote $\lambda=s_{12} \approx 0.22$. Experiments indicate that $s_{23}=O\left(\lambda^{2}\right)$ and $s_{13}=O\left(\lambda^{3}\right)$. Setting $s_{23}=A \lambda^{2}$ and $s_{13} e^{-i \delta}=A \lambda^{2}(\rho-i \eta)$ an expansion of the CKM matrix up to $O\left(\lambda^{4}\right)$ gives the Wolfenstein parametrisation [13]

$$
\mathbf{V}_{C K M}=\left(\begin{array}{ccc}
1-\lambda^{2} / 2 & \lambda & A \lambda^{3}(\rho-i \eta) \\
-\lambda & 1-\lambda^{2} / 2 & A \lambda^{2} \\
A \lambda^{3}(1-\rho-i \eta) & -A \lambda^{2} & 1
\end{array}\right)+\mathcal{O}\left(\lambda^{4}\right)
$$

This parameterisation is often used in $B$ physics. In this parameterisation $J_{C P} \simeq$ $A^{2} \eta \lambda^{6} \simeq 1.1 \times 10^{-4} A^{2} \eta$, for $\lambda \simeq 0.22$.

\subsubsection{The Unitarity Triangle}

The unitary condition $\mathbf{V}_{C K M} \mathbf{V}_{C K M}^{\dagger}=1$ implies various relations among the elements of the CKM matrix

$$
V_{i j} V_{j k}^{*}=0, \quad i \neq k
$$

These can be represented as closed triangles on a complex plane, so called "Unitarity Triangles". Each of these triangles share the same area $\frac{1}{2} J_{C P}$. The most useful triangle from the perspective of $B$ physics is

$$
V_{u d} V_{u b}^{*}+V_{c d} V_{c b}^{*}+V_{t d} V_{t b}^{*}=0,
$$

and is shown in Fig. 1.1. It is standard practise to renormalise the sides of the triangle by $V_{c d} V_{c b}^{*}$ (also shown in Fig. 1.1) so that the corners of the triangle are at $(0,0),(0,1)$ and $(\bar{\rho}, \bar{\eta})$, where

$$
\bar{\rho}=\left(1-\frac{\lambda^{2}}{2}\right) \rho, \quad \bar{\eta}=\left(1-\frac{\lambda^{2}}{2}\right) \eta .
$$

The length of the $V_{u d} V_{u b}^{*}$ side of the rescaled triangle $\left(R_{b}\right)$ is equal to $\sqrt{\bar{\rho}^{2}+\bar{\eta}^{2}}$. $R_{t}$, the length of the $V_{t d} V_{t b}^{*}$ side, is given by $R_{t}=\sqrt{(\bar{\rho}-1)^{2}+\bar{\eta}^{2}}$. In terms of the $\mathbf{V}_{C K M}$ elements the angles of the unitarity triangle are

$$
\alpha=\arg \left[-\frac{V_{t d} V_{t b}^{*}}{V_{u d} V_{u b}^{*}}\right], \quad \beta=\arg \left[-\frac{V_{c d} V_{c b}^{*}}{V_{t d} V_{t b}^{*}}\right], \quad \gamma=\arg \left[-\frac{V_{u d} V_{u b}^{*}}{V_{c d} V_{c b}^{*}}\right] .
$$




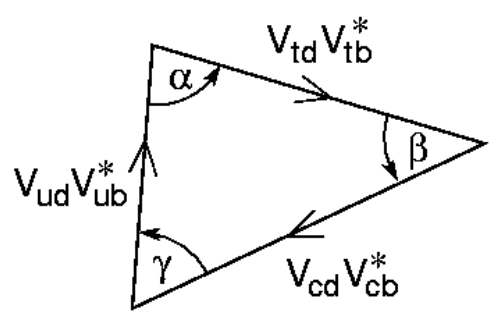

(a)

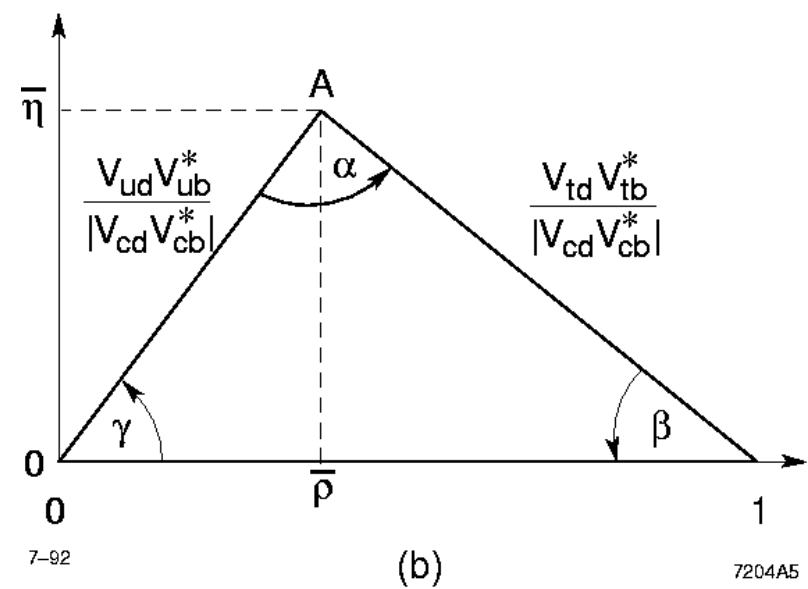

Figure 1.1: The unitarity triangle and its renormalised version

By measuring the angles and lengths of this triangle we can test the constraint that $\mathbf{V}_{C K M}$ be unitary and all of the implications this has for the Standard Model.

\subsubsection{Constraining the CKM Matrix}

The accuracy to which the CKM parameters are known experimentally varies to a large extent. The first two rows can in principle be extracted from tree level processes i.e. weak decays involving the corresponding quark. The current values and measurement techniques are summarised in the following. All numbers are taken from $[7]$

- $\left|V_{u d}\right|$ : This is measured using nuclear beta decays. The average value is

$$
\left|V_{u d}\right|=0.9734 \pm 0.0008 .
$$

- $\left|V_{u s}\right|$ : This is extracted from semileptonic kaon decays $\left(K_{e 3}\right)$ and hyperon beta decay. The theoretical errors for hyperon decay are much larger than those 
for $K_{3 e}$ decays which yields the value

$$
\left|V_{u s}\right|=0.2196 \pm 0.0026 \text {. }
$$

- $\left|V_{c d}\right|$ : The magnitude of $\left|V_{c d}\right|$ is deduced from neutrino and antineutrino production of charm off valence quarks. Averaging results from the CDHS group and the CCFR at the Tevatron gives

$$
\left|V_{c d}\right|=0.224 \pm 0.0016
$$

- $\left|V_{c s}\right|$ : The value of $\left|V_{c s}\right|$ can be measured by semileptonic D decays, with theoretical uncertainty of around $10 \%$. These measurements have been superseded by a direct measurement of $\left|V_{c s}\right|$ in charm tagged $\mathrm{W}$ decays that gives

$$
\left|V_{c s}\right|=0.97 \pm 0.09 \pm 0.07 \text {. }
$$

A tighter determination can be made using the unitarity condition of the CKM matrix and the ratio of hadronic $\mathrm{W}$ decays to leptonic decays which yields

$$
\left|V_{c s}\right|=0.996 \pm 0.013 \text {. }
$$

- $\left|V_{c b}\right|$ : Heavy quark effective theory (HQET) provides a model independent treatment of semileptonic $B$ decays to charm quarks. Key to this theory is the mass of the quarks and it requires that the $b$ and $c$ quarks are of sufficient mass for the theory to apply $\left(m_{q}>>\Lambda_{Q C D}\right)$. In this limit the decay can be parametrised in terms of the velocity of the heavy quark. The spin and flavour of the heavy quark become irrelevant. Measurement of exclusive decays $B \rightarrow D^{*} l^{+} \nu$ can be used to extract a value of $\left|V_{c b}\right|$. Inclusive measurements of semileptonic $B$ decays rely on the assumption of heavy quark duality. Averaging exclusive and inclusive results gives

$$
\left|V_{c b}\right|=(41.2 \pm 2.0) \times 10^{-3} .
$$

$B A B A R$ have recently released a preliminary result for $\left|V_{c b}\right|$ measured using exclusive $B \rightarrow D^{*} l^{+} \nu$ decays. They report a result

$$
\left|V_{c b}\right| F(1)=(34.24 \pm 0.24 \pm 1.30) \times 10^{-3} .
$$

where $F(1)$ is the form factor when the $D^{*}$ is produced at rest in the $B^{0}$ rest frame and is estimated to be $F(1)=0.91 \pm 0.03$. 
- $\left|V_{u b}\right|$ : The measurement of $\left|V_{u b}\right|$ is explained in more detail in the following sections. For now we will just give the result from averaging results from semileptonic B decays

$$
\left|V_{u b}\right|=(3.6 \pm 0.7) \times 10^{-3} .
$$

- $\left|V_{t b}\right|$ : The recent discovery of the top quark utilised in part the semileptonic decay $t \rightarrow b$. The CDF experiment has published a limit in the ratio of the semileptonic decays $\mathrm{t} \rightarrow \mathrm{b}$, compared to $\mathrm{t} \rightarrow$ other quarks

$$
\frac{\left|V_{t b}\right|^{2}}{\left|V_{t d}\right|^{2}+\left|V_{t s}\right|^{2}+\left|V_{t b}\right|^{2}}=0.94_{-0.24}^{+0.31} .
$$

Using these results and assuming unitarity, for 3 generations of quarks the $90 \%$ confidence limits in the complete matrix are

$$
\mathbf{V}_{C K M}=\left(\begin{array}{ccc}
0.9741-0.9756 & 0.219-0.226 & 0.0025-0.0048 \\
0.219-0.226 & 0.9732-0.9748 & 0.038-0.44 \\
0.004-0.014 & 0.037-0.044 & 0.9990-0.9993
\end{array}\right)
$$

These measurements together with indirect measurements can be used to constrain the CKM matrix. The unitarity triangle provides a useful framework by which this can be carried out. The shape of the triangle can be determined by measuring the side and angles.

- The length of the side $R_{b}$ is given by

$$
R_{b}=\left(1-\frac{\lambda^{2}}{2}\right) \frac{1}{\lambda}\left|\frac{V_{u b}}{V_{c b}}\right| .
$$

- To determine the $R_{t}$ we need to determine a value of $\left|V_{t d}\right|$,

$$
R_{t}=\frac{1}{\lambda}\left|\frac{V_{t d}}{V_{c b}}\right| .
$$

This can be extracted from $B^{0}-\bar{B}^{0}$ mixing. The mass difference between $B^{0}$ and $\bar{B}^{0}$ is given by

$$
\Delta m_{B}=\frac{G_{F}^{2} m_{W}^{2}}{6 \pi^{2}} \eta_{B} B_{B} f_{B}^{2} m_{B} S\left(m_{t} / m_{w}\right)\left|V_{t d} V_{t b}^{*}\right|^{2} .
$$


By taking the ratio of the $B_{s}$ to $B_{d}$ mass difference many of the common constants cancel, including the QCD correction $\eta_{B}$ and the dependance on the top quark mass. This gives

$$
\frac{\Delta m_{B_{s}}}{\Delta m_{B_{d}}}=\frac{B_{B_{s}} f_{B_{s}}^{2}}{B_{B_{d}} f_{B_{d}}^{2}} \frac{m_{B_{s}}}{m_{B_{d}}} \frac{\left|V_{t s} V_{t b}^{*}\right|^{2}}{\left|V_{t d} V_{t b}^{*}\right|^{2}} .
$$

The angles of the unitarity triangle correspond to physical quantities and can be measured. In the $C P$-violating asymmetries of neutral $B$ mesons decaying to $C P$ eigenstates, the interference between mixing and a single weak decay amplitude for some final states is directly related to $\sin 2 \phi$, where $\phi$ is one of the angles $\alpha, \beta$ or $\gamma$.

- The decay $B^{0} \rightarrow J / \psi K_{S}^{0}$ is considered to be the "gold plated" mode for the measurement of $\sin 2 \beta$. The asymmetry in this decay can be related to

$$
\lambda_{\left(\psi K_{S}^{0}\right)}=\frac{V_{t d}^{*} V_{t d}}{V_{t b} V_{t d}^{*}} \cdot \frac{V_{c s} V_{c d}^{*}}{V_{c b}^{*} V_{c d}} \cdot \frac{V_{c b} V_{c s}^{*}}{V_{c b}^{*} V_{c s}}=-e^{-2 i \beta}
$$

where $\lambda$ corresponds to that in Eq. 1.14. The first term is due to $B^{0}-\bar{B}^{0}$ mixing, the second term is from the charged weak current and the last term is from $K^{0}-\bar{K}^{0}$ mixing. Hence

$$
\sin 2 \beta \simeq \operatorname{Im}\left(\lambda_{\left(\psi K_{S}^{0}\right)}\right) .
$$

This situation is potentially complicated as the decays can proceed via the tree level diagram or a so-called penguin diagram, both of which are shown in 1.2. In the case of $B^{0} \rightarrow J / \psi K_{S}^{0}$ the same weak phase appears in the penguin and tree contribution and the penguin contribution is much smaller than the tree diagram. The theoretical uncertainties are very small and this is the reason why this decay is considered the "gold plated" mode for measuring $\sin 2 \beta$. This angle has been recently measured by BABAR and BELLE. The current $B A B A R$ result is $\sin 2 \beta=0.741 \pm 0.067$ (stat) \pm 0.034 (syst) [14].

- The angle $\sin 2 \alpha$ can also be measured in this way, only here there is not as much certainty as to the significance of the penguin contribution. This measurement is also complicated because of the small branching fraction of the decay modes involved. 

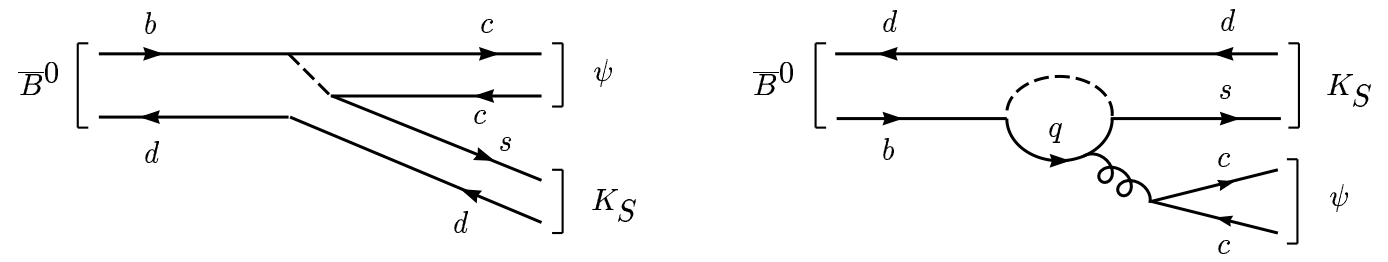

Figure 1.2: The tree (left) and penguin (right) Feynman diagram for the decay $B^{0} \rightarrow J / \psi K_{S}^{0}$.

Further constraints on the unitarity triangle are provided by $K^{0}-\bar{K}^{0}$. The present status of the total combined constraints is shown in Fig. 1.3. This shows the $(\bar{\rho}, \bar{\eta})$ plane with the allowed region for the apex of the unitarity triangle. The plot has been made using [15]

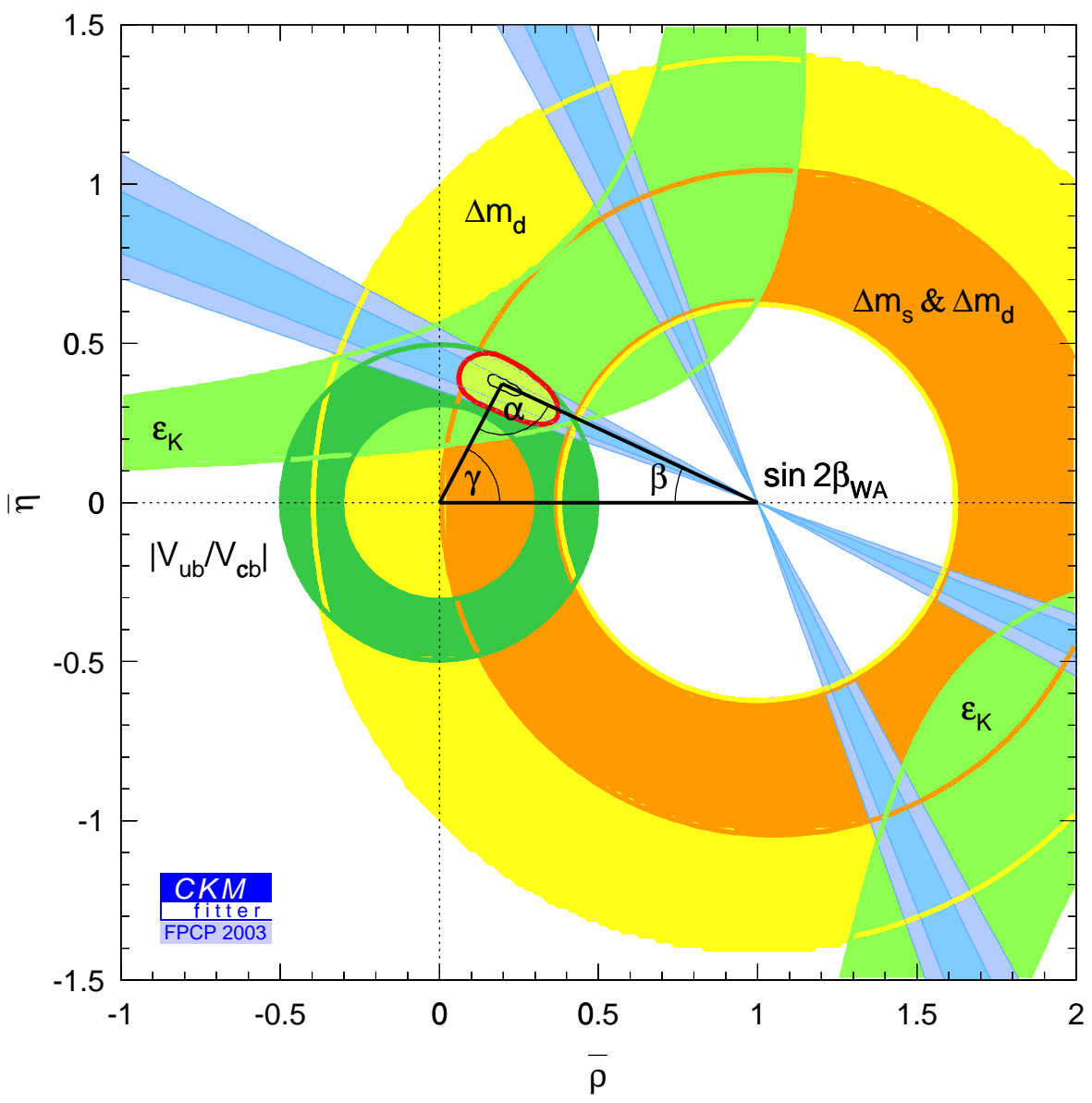

Figure 1.3: The plot shows the $(\bar{\rho}, \bar{\eta})$ plane with the current constraints on the CKM matrix. The unitarity triangle is superimposed on the plot. 


\subsection{Measuring $\mathrm{V}_{\mathrm{ub}}$ at $B A B A R$}

\subsubsection{Introduction}

This section outlines some of the theoretical tools necessary to describe the semileptonic decay of a $B$ meson to a charmless meson and the techniques used to extract $\left|V_{u b}\right|$ from this process. In the case of $\left|V_{c b}\right|$ with CKM-favoured decays $b \rightarrow c l \nu$, the relatively model-independent HQET can be used to determine the matrix element for the decay. This is not the case for charmless decays. A number of different models can be employed, the theoretical errors for which currently lie in the 10$20 \%$ range. A more in-depth descussion on the subject of this section can be found in $[7,8]$.

\subsubsection{Decay Kinematics}

The Feynman diagram for semileptonic $B$ decays is shown in Fig. 1.4. The matrix

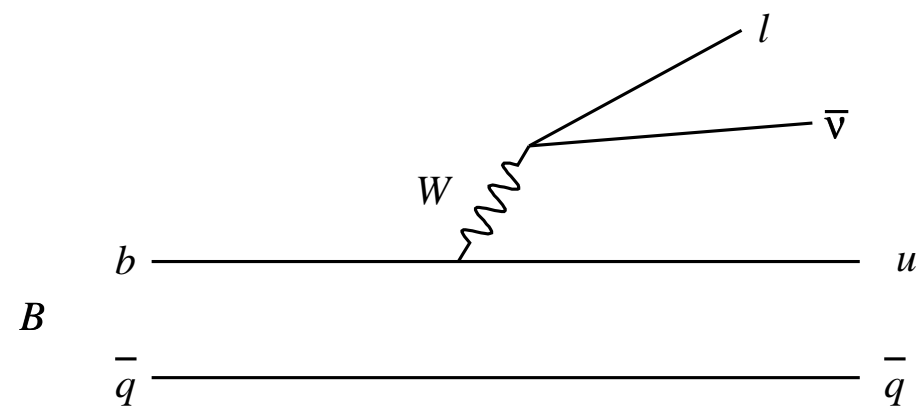

Figure 1.4: Feynman diagram for charmless semileptonic decay of a $B$ meson.

element for the semileptonic decay of a pseudoscalar meson $M_{Q \bar{q}}$ into a meson $X_{q^{\prime} \bar{q}}$ is given by

$$
\mathcal{M}\left(M_{Q \bar{q}} \rightarrow X_{q^{\prime} \bar{q}} l^{-} \bar{\nu}\right)=-i \frac{G_{F}}{\sqrt{2}} V_{q^{\prime} Q} L^{\mu} H_{\mu}
$$


where $G_{F} / \sqrt{2}=g^{2} /\left(8 M_{W}^{2}\right), L^{\mu}=\bar{u}_{l} \gamma^{\mu}\left(1-\gamma^{5}\right) v_{\nu}$ is the leptonic current in terms of the Dirac spinors $u_{l}$ and $v_{\nu}, H_{\mu}=\left\langle X_{q^{\prime}}\left|\bar{q}^{\prime} \gamma^{\mu}\left(1-\gamma_{5}\right) Q\right| M_{Q \bar{q}}\right\rangle$ is the hadronic current and $V_{q^{\prime} Q}$ is the relevant element of the CKM matrix. While the leptonic current is simple to evaluate, the hadronic current is complicated by strong interactions. The strong interactions in the initial and final states of the meson are separated into form factors. The form factors that enter into these equations depend on the spin of the final meson. To ensure Lorentz invariance the hadronic current must be constructed from the available four-vectors which are the momenta of the two mesons and the spin-polarisation vector of the daughter meson.

\section{Decays to pseudoscalar mesons: e.g. $\mathbf{B} \rightarrow \pi \mathbf{l} \nu$.}

In this case there are only two independent four-vectors which we can take to be $p+p^{\prime}$ and $q=p-p^{\prime}$ where $p$ is the four momentum of the $B$ meson and $p^{\prime}$ is the four momentum of the daughter meson. In this case the hadronic current has no axial vector contribution and can be generally written in terms of two form factors as $f^{+}\left(q^{2}\right)$ and $f^{-}\left(q^{2}\right)$ as $[7,8]$

$$
\begin{aligned}
\left\langle\pi\left(p^{\prime}\right)\left|\bar{b} \gamma^{\mu} u\right| B(p)\right\rangle= & f^{+}\left(q^{2}\right)\left[\left(p+p^{\prime}\right)^{\mu}-\frac{M_{B}^{2}-m_{\pi}^{2}}{q^{2}} q^{\mu}\right] \\
& +f^{-}\left(q^{2}\right) \frac{M_{B}^{2}-m_{\pi}^{2}}{q^{2}} q^{\mu} .
\end{aligned}
$$

where $f^{+}(0)=f^{-}(0)$. This equation can be simplified by making the approximation of massless leptons. In the limit $m_{l} \rightarrow 0, q^{\mu} L_{\mu}=0$ so terms proportional to $q^{\mu}$ can be neglected. The amplitude now only depends upon one form factor $f^{+}\left(q^{2}\right)$. The differential decay rate is given by

$$
\frac{d \Gamma}{d q^{2}}=\frac{G_{F}^{2}\left|V_{u b}\right|^{2} p_{\pi}^{3}}{24 \pi^{3}}\left|f^{+}\left(q^{2}\right)\right|^{2},
$$

where $p_{\pi}^{3}$ is the momentum of the daughter meson in the centre of mass frame of the $B$. 


\section{Decays to vector mesons: e.g. $\mathbf{B} \rightarrow \rho \mathbf{l} \nu$.}

We now have to also consider the polarisation vector of the vector meson $(\varepsilon)$. The hadronic current must be linear in $\varepsilon$ and can be generally written as $[7,8]$

$$
\begin{aligned}
\left\langle\rho\left(p^{\prime}, \varepsilon\right)\left|V^{\mu}-A^{\mu}\right| B(p)\right\rangle & =\frac{2 i V\left(q^{2}\right)}{M_{B}+m_{\rho}} \epsilon^{\mu \nu \alpha \beta} \varepsilon_{\nu}^{*} p_{\alpha}^{\prime} p_{\beta} \\
& -\left(M_{B}+m_{\rho}\right) A_{1}\left(q^{2}\right) \varepsilon^{* \mu}+\frac{A_{2}\left(q^{2}\right)}{M_{B}+m_{\rho}} \varepsilon^{*} \cdot q\left(p+p^{\prime}\right)^{\mu} \\
& +2 A_{3}\left(q^{2}\right) m_{\rho} \frac{\varepsilon^{*} \cdot q}{q^{2}} q^{\mu}-2 A_{0}\left(q^{2}\right) m_{\rho} \frac{\varepsilon^{*} \cdot q}{q^{2}} q^{\mu}
\end{aligned}
$$

where $V^{\mu}=\bar{b} \gamma^{\mu} u, A^{\mu}=\bar{b} \gamma^{\mu} \gamma_{5} u, A_{0}(0)=A_{3}(0)$ and

$$
A_{3}=\frac{M_{B}+m_{\rho}}{2 m_{\rho}} A_{1}-\frac{M_{B}-m_{\rho}}{2 m_{\rho}} A_{2} .
$$

Again the assumption of massless leptons can be used, removing terms proportional to $q^{\mu}$. The hadronic current then depends on three form factors $A_{1}\left(q^{2}\right), A_{2}\left(q^{2}\right)$ and $V\left(q^{2}\right)$. The decay rates are usually written in terms of helicity amplitudes

$$
\begin{aligned}
H_{ \pm}\left(q^{2}\right)= & \left(M_{B}+m_{\rho}\right) A_{1}\left(q^{2}\right) \mp \frac{2 M_{B}\left|\vec{p}_{\rho}\right|}{M_{B}+m_{\rho}} V\left(q^{2}\right), \\
H_{0}\left(q^{2}\right)= & \frac{1}{2 m_{\rho} \sqrt{q^{2}}}\left[\left(M_{B}^{2}-m_{\rho}^{2} q^{2}\right)\left(M_{B}+m_{\rho}\right) A_{1}\left(q^{2}\right)\right. \\
& -4 \frac{M_{B}^{2}\left|\vec{p}_{\rho}\right|^{2}}{M_{B}+m_{\rho}} A_{2}\left(q^{2}\right),
\end{aligned}
$$

The differential decay rate for the vector meson decaying to two pseudoscalar mesons is then given by

$$
\begin{aligned}
\frac{d \Gamma(B \rightarrow \rho \ell \nu, \rho \rightarrow \pi \pi)}{d q^{2} d \cos \theta_{\rho} d \cos \theta_{\ell} d \chi}= & \frac{3}{8(4 \pi)^{4}} G_{F}^{2}\left|V_{u b}\right|^{2} \frac{\left|\vec{k}_{\rho}\right| q^{2}}{M_{B}^{2}} B(\rho \rightarrow \pi \pi) \\
\times & \left\{\left(1-\zeta \cos \theta_{\ell}\right)^{2} \sin \theta_{\rho}{ }^{2}\left|H_{+}\left(q^{2}\right)\right|^{2}\right. \\
+ & \left(1+\zeta \cos \theta_{\ell}\right)^{2} \sin \theta_{\rho}{ }^{2}\left|H_{-}\left(q^{2}\right)\right|^{2} \\
+ & 4 \sin \theta_{\ell}{ }^{2} \cos \theta_{\rho}{ }^{2}\left|H_{0}\left(q^{2}\right)\right|^{2} \\
- & 4 \zeta \sin \theta_{\ell}\left(1-\zeta \cos \theta_{\ell}\right) \sin \theta_{\rho} \cos \theta_{\rho} \cos \chi H_{+}\left(q^{2}\right) H_{0}\left(q^{2}\right) \\
- & 4 \zeta \sin \theta_{\ell}\left(1+\zeta \cos \theta_{\ell}\right) \sin \theta_{\rho} \cos \theta_{\rho} \cos \chi H_{-}\left(q^{2}\right) H_{0}\left(q^{2}\right) \\
- & \left.2 \sin \theta_{\ell}{ }^{2} \sin \theta_{\rho}{ }^{2} \cos 2 \chi H_{+}\left(q^{2}\right) H_{-}\left(q^{2}\right)\right\},
\end{aligned}
$$




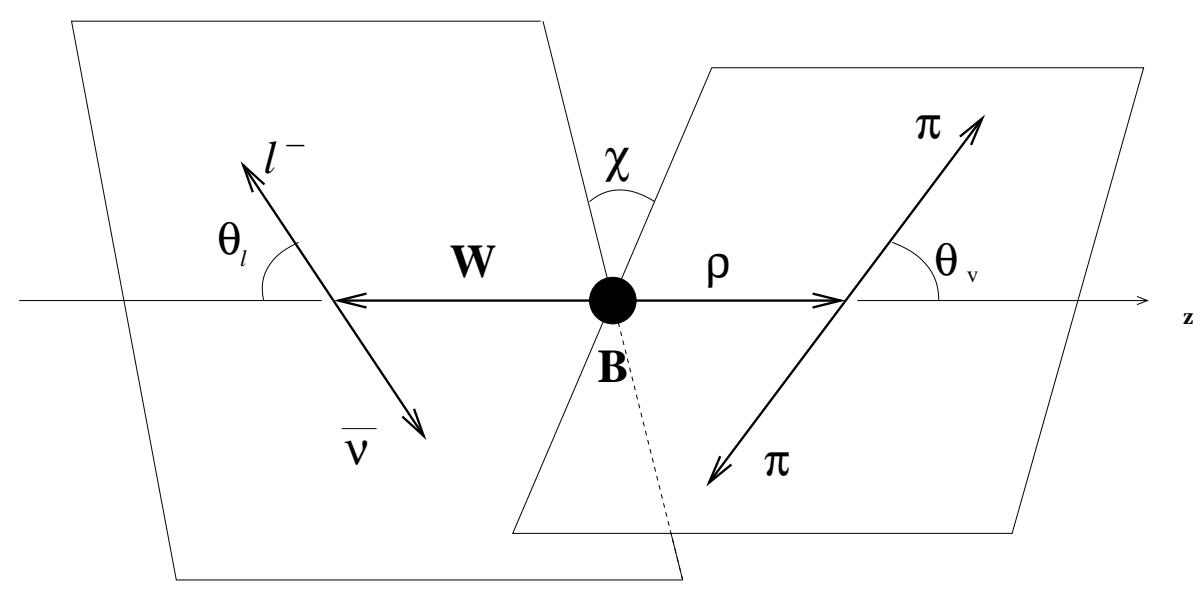

Figure 1.5: The definition of the angles that occur in the semileptonic decay of a $B$ meson to a vector meson, which subsequently decays to two pseudoscalar mesons.

where the angles are defined in Fig. 1.5. This rate can be integrated over the angular variables to give

$$
\frac{d \Gamma}{d q^{2}}=\frac{G_{F}^{2}}{96 \pi^{3}}\left|V_{u b}\right|^{2} \frac{p_{\rho} q^{2}}{m_{B}^{2}} B(\rho \rightarrow \pi \pi)\left[\left|H_{+}\left(q^{2}\right)\right|^{2}+\left|H_{-}\left(q^{2}\right)\right|^{2}+\left|H_{0}\left(q^{2}\right)\right|^{2}\right] .
$$

\subsubsection{Theoretical Models for Form Factors}

There are a variety of models which are commonly used to describe the form factors occurring in charmless semileptonic $B$ decays. Light Cone Sum Rules (LCSR) and lattice calculations are both based on QCD and currently have uncertainties in the $15 \%$ to $20 \%$ range. There are also a number of quark models, such as ISGW2 which is used as the default for production of non-resonant hadronic states and all charmless semileptonic decays in BABAR. The models usually predict the values of the form factors at a particular value of $q^{2}$ and then extrapolate the form factors over the full $q^{2}$ range as a separate step.

The form factors are needed to provide the normalisation for the determination of $\left|V_{u b}\right|$ from the measured branching fraction, $\mathcal{B}$,

$$
\left|V_{u b}\right|=\sqrt{\frac{\mathcal{B}}{\tilde{\Gamma}_{t h y} \tau_{B^{0}}}}
$$


where $\tau_{B^{0}}$ is the $B$ lifetime and $\tilde{\Gamma}_{t h y}$ is the partial width excluding CKM factors provided by theory. In order to reduce the background from $b \rightarrow c l \nu$ decays we limit the region of lepton momentum. Hence form factors are required to relate the measured branching fraction in the limited phase space region to the full rate.

\section{ISGW2}

This is a constituent quark model purposed by Isgur, Scora, Grinstein and Wise [16] (and their later update [17]). The calculations are normalised at $q^{2}=q_{m a x}^{2}$. There is no means to systematically evaluate the theoretical uncertainties for this approach. The authors estimate an uncertainty of around $50 \%$.

\section{Lattice QCD}

The lattice QCD technique (e.g. [18]) is based on QCD and can potentially yield a model independent determination of the form factors from first principles. In practice however, lattice calculations conducted so far have been in the quenched approximation (virtual quark loops are removed from the calculation). This ultimately limits the uncertainties to the $15 \%$ to $20 \%$ range. Lattice calculations provide values of the form factors over a limited range at high $q^{2}$.

\section{LCSR}

LCSR [19] are based on non-perturbative calculations. They are most predictive at low values of $q^{2}$ and thus provide complementary measurement to the lattice QCD techniques. The results from LCSR and lattice QCD overlap at intermediate values of $q^{2}$, which allows consistency between the methods to be investigated. The LCSR technique relies on quark-hadron duality that contributes an uncertainty of $10 \%$, but with no means of rigourously limiting this uncertainty. 


\subsubsection{Inclusive Measurements}

So far the discussion has been focused on exclusive semileptonic decays only. It is also possible to extract a measure of $\left|V_{u b}\right|$ by measuring the inclusive rate $B\left(b \rightarrow X_{u} l \bar{\nu}\right)$. In principle this rate can be calculated in the Operator Product Expansion (OPE) framework, with $\mathrm{a} \simeq 6 \%$ theoretical uncertainty. Problems occur when the region of total phase space is decreased to limit backgrounds from $b \rightarrow c l \bar{\nu}$ decays. The three regions generally used in experiment are the lepton end point region $E_{l}>$ $\left(M_{B}^{2}-M_{D}^{2}\right) /\left(2 M_{B}\right)$, the low hadronic mass region $M_{X}<M_{D}$ and the large leptonic mass region $q^{2}>\left(M_{B}-M_{D}\right)$. While the necessary approximation of quark-hadron duality is expected to hold for the global phase space, it is not guaranteed to hold for the partially integrated rates. The theoretical uncertainties caused are the subject of on-going debate. Inclusive measurements have a typical signal to background ratio (S/B) a factor of 5 less than those achieved in exclusive analyses. They are therefore more affected by systematic uncertainties from the description of the $b \rightarrow$ $c$ background.

\subsubsection{Previous Measurements of $\left|V_{u b}\right|$}

The $\left|V_{u b}\right|$ CKM matrix element was first shown to be non-zero by CLEO [20] and ARGUS [21] over a decade ago. Since then great strides have been made in both experimental and theoretical understanding. The present experimental standing is illustrated in Tables 1.1(exclusive measurements) and 1.2 (inclusive measurments which is taken from [22]).

\subsection{Current Analysis}

This thesis presents exclusive measurements of the branching fractions $B \rightarrow X_{u} l \nu$, where $X_{u}$ is one of either the pseudoscalar mesons $\pi^{-}$or $\pi^{0}$ or the vector mesons $\rho^{-}, \rho^{0}$ or $\omega$. A measurement of $\left|V_{u b}\right|$ is then extracted using the measured branching fractions. 


\begin{tabular}{ccc}
\hline Experiment & Method & $\left|V_{u b}\right|\left[10^{-3}\right]$ \\
\hline CLEO [23] & $\rho l \nu+\operatorname{loose} \nu$ & $3.23 \pm 0.24_{-0.26}^{+0.23} \pm 0.58$ \\
\hline BABAR [24] & $\rho l \nu+$ loose $\nu$ & $3.64 \pm 0.22 \pm 0.25_{-0.56}^{+0.39}$ \\
\hline CLEO [25] & $\pi l \nu+$ tight $\nu$ & $3.24 \pm 0.22 \pm 0.13 \pm 0.09_{-0.39}^{+0.55}$ \\
CLEO [25] & $\rho l \nu+$ tight $\nu$ & $3.00 \pm 0.21_{-0.35}^{+0.29} \pm 0.28_{-0.38}^{+0.49}$ \\
\hline Belle [26] & $\pi l \nu$ LCSR & $3.11 \pm 0.13 \pm 0.24 \pm 0.56$ \\
Belle [26] & $\pi l \nu$ UKQCD & $3.58 \pm 0.15 \pm 0.28 \pm 0.63$ \\
Belle [26] & $\rho l \nu$ ISGW2 & $3.50 \pm 0.20 \pm 0.28$ \\
\hline
\end{tabular}

Table 1.1: Summary of $\left|V_{u b}\right|$ exclusive measurements by experiment. The table gives the result along with the decay mode studied and the method used (tight $\nu$ indicates that tight neutrino reconstruction has been employed). For the first two results the errors are statistical, experimental systematic and form factor modelling. The next two CLEO results give statistical, experimental systematic, $\rho l \nu$ form factor shape, and form factor normalisation errors. The first two Belle results quote statistical, experimental systematic and form factor model uncertainties, while the final result just gives statistical and experimental systematic errors.

\begin{tabular}{ccc}
\hline Experiment & $\left|V_{u b}\right|\left[10^{-3}\right]$ & Method \\
\hline ALEPH [27] & $4.12 \pm 0.67 \pm 0.76$ & Neural Net \\
OPAL [28] & $4.00 \pm 0.71 \pm 0.71$ & Neural Net \\
DELPHI [29] & $4.07 \pm 0.65 \pm 0.61$ & $m_{X}$ \\
L3 [30] & $5.7 \pm 1.0 \pm 1.4$ & cut and count \\
LEP Average [7] & $4.09 \pm 0.37 \pm 0.56$ & \\
\hline \hline CLEO & $4.08 \pm 0.22 \pm 0.61$ & End point \\
BABAR & $4.43 \pm 0.26 \pm 0.67$ & End point \\
\hline \hline
\end{tabular}

Table 1.2: Summary of $\left|V_{u b}\right|$ inclusive measurements by experiment. The result, with experimental and theoretical errors are given, along with the method used. This table is taken from [22]. 
The ISWG2 model is used to describe the decays of the signal channels and to extract the value of $\left|V_{u b}\right|$. 


\section{Chapter 2}

\section{The PEP-II Storage Ring and the BABAR Detector}

\section{$2.1 \quad$ Introduction}

PEP-II is an asymmetric $B$ Factory which sits at the $\Upsilon(4 S)$ resonance and operates at SLAC. The purpose of the PEP-II facility is to over-constrain the CKM matrix by measuring CKM matrix phases and magnitudes using $B$ meson decays. The BABAR experiment is part of this program. The primary goal of the BABAR experiment is the systematic study of time-dependent $C P$ asymmetries in neutral $B$ mesons. The $B A B A R$ detector is therefore optimised to measure neutral $B$ meson decays to $C P$ eigenstates. Such a detector is required to:

- reconstruct $B$ meson decays to a wide variety of exclusive final states,

- tag the flavour of the other $B$ meson in the event,

- reconstruct the relative decay time of the two $B$ mesons.

The secondary goal of the $B A B A R$ experiment is the precision measurement of $B$ meson, charmed meson and $\tau$ decays, and, making use of the high luminosity, the search for rare decays. 
This chapter will go through the various components of the PEP-II storage ring and the $B A B A R$ detector, outlining the physics requirements and capabilities.

Further information on the physics goals of the $B A B A R$ detector can be found in the BABAR Letter of Intent [31] and the Technical Design Report [32]. Details of the $B A B A R$ detector can be found in [33]. The PEP-II storage ring is described in [34].

\subsection{The PEP-II Storage Ring}

PEP-II is an $e^{+} e^{-}$storage ring designed to operate at the $\Upsilon(4 S)$ resonance, which corresponds to a centre of mass energy of $10.58 \mathrm{GeV}$. The $\Upsilon(4 S)$ resonance is just above the threshold for $B \bar{B}$ production and decays to $B \bar{B}$ at least $96 \%$ of the time. $9.0 \mathrm{GeV} e^{-}$are collided with $3.1 \mathrm{GeV} e^{+}$, resulting in a boost of $\beta \gamma=0.56$. While most of the data are taken at $10.58 \mathrm{GeV}, 12 \%$ are taken at an energy $40 \mathrm{MeV}$ below the $\Upsilon(4 S)$ resonance. This is to provide data for non-resonant background studies, i.e. all decays that do not proceed through the $\Upsilon(4 S)$ resonance. The value of $12 \%$ is optimised to give the smallest statistical error for CP asymmetry measurements.

\subsubsection{Beam Parameters}

Table 2.1 lists some of the PEP-II design parameters and their typical values during the first year of running. The best values so far acheived are also given. PEP-II has surpassed its design goals, both in terms of instantaneous and daily integrated luminosity, even with fewer bunches than anticipated. A BABAR run corresponds typically to a 40-50 minute cycle during which the conditions are deemed to be stable. Between runs there is a 3 minute top up time. It takes 10-15 minutes to refill the beams after a beam loss. 


\begin{tabular}{l||c|c|c}
\hline \hline Parameters & Design & Typical & Best \\
\hline Energy HER/LER (GeV) & $9.0 / 3.1$ & $9.0 / 3.1$ & $9.0 / 3.1$ \\
Current HER (A) & 0.75 & 0.7 & 1.18 \\
Current LER (A) & 2.15 & 1.3 & 1.55 \\
\# bunches & 1658 & $553-829$ & 1034 \\
bunch spacing (ns) & 4.2 & $6.3-10.5$ & \\
$\sigma_{L_{x}}(\mu \mathrm{m})$ & 110 & 120 & \\
$\sigma_{L_{y}}(\mu \mathrm{m})$ & 3.3 & 5.6 & \\
$\sigma_{L_{z}}(\mathrm{~mm})$ & 9 & 9 & \\
Luminosity $\left(10^{33} \mathrm{~cm}^{-2} \mathrm{~S}^{-1}\right)$ & 3 & 2.5 & 6.6 \\
Luminosity $(\mathrm{pb} / \mathrm{day}$ & 135 & 120 & 395 \\
\hline \hline
\end{tabular}

Table 2.1: Parameters of the PEP-II machine. The typical values are the average values achieved during the first year of running. The third column gives the best values achieved to date

\subsubsection{Interaction Point}

At the Interaction Point (IP) the beams need to be collided head-on and then separated quickly to avoid parasitic collisions. This is achieved using a pair of dipole magnets (B1) located $\pm 21 \mathrm{~cm}$ either side of the IP, followed by a series of offset quadrupoles. The area around the IP is shown in Fig. 2.1. The B1 dipoles and Q1 quadrupole are permanent magnets made from samarium-cobalt. As the Q2, Q4 and Q5 quadrupoles are further out of the BABAR solenoid magnetic field, standard iron magnets can be used. The IP is enclosed by a $27.9 \mathrm{~mm}$ radius beam pipe consisting of two layers of beryllium with water cooling between the layers. The beam pipe corresponds to $1.06 \%$ of a radiation length at normal incidence. The beam pipe and magnets are enclosed by a $4.5 \mathrm{~m}$ long support pipe which covers the IP.

\subsubsection{Machine Backgrounds}

The primary sources of background, in decreasing order of significance, are synchrotron radiation, beam-gas collisions and electromagnetic showers from beambeam collisions. The synchrotron radiation generated by the magnets near the IP region is controlled by orbit design and synchrotron radiation masks. The beam-gas background is generated by beam interactions with gas particles inside the beam 
PEP-II Interaction Region

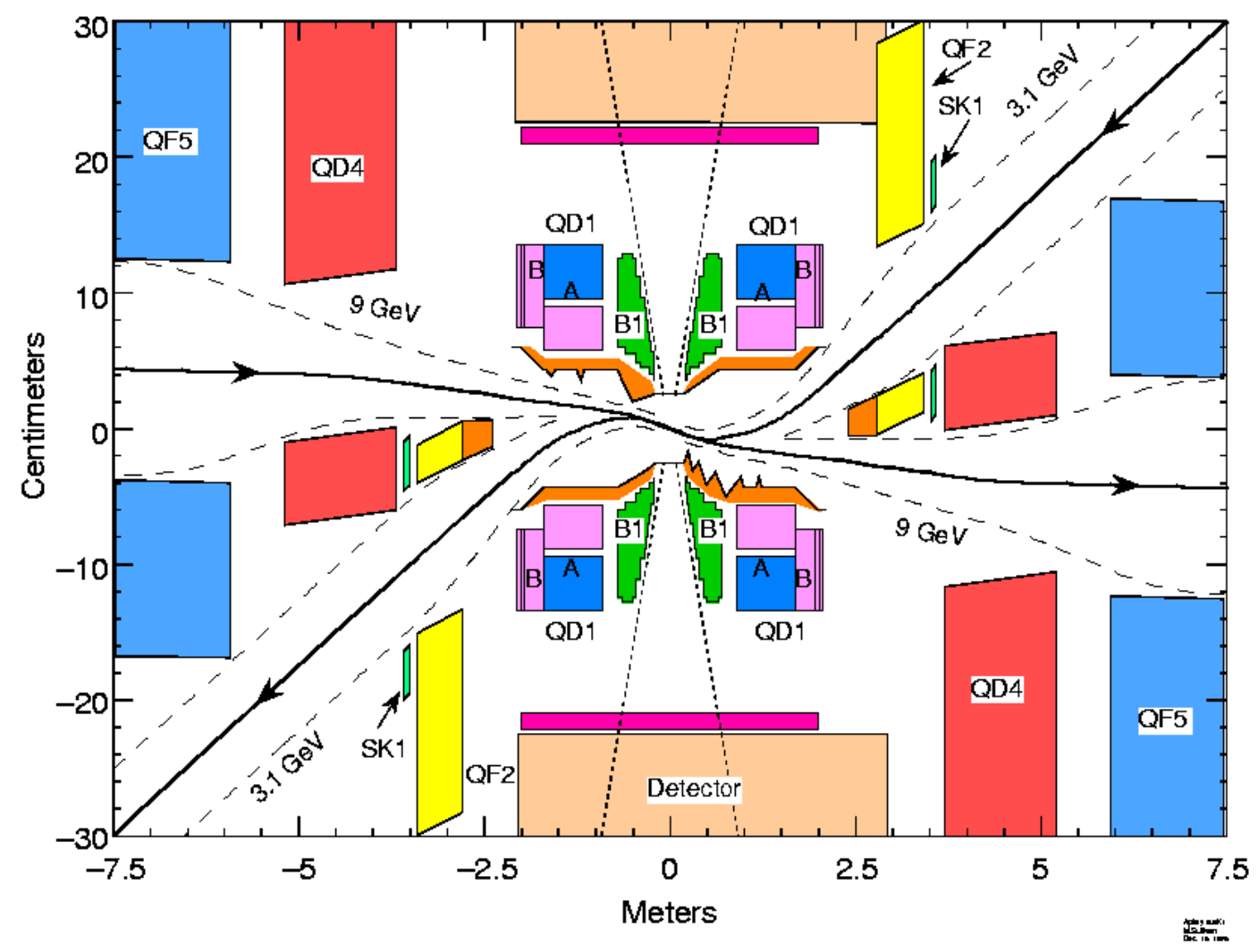

Figure 2.1: A schematic of the PEP-II elements at the BABAR IP.

pipe. The rate of this background is proportional to the current in the beam and the pressure in the beam pipe. This is the primary source of radiation in the silicon vertex tracker and dominant source in all other detector subsystems apart from the detector of internally reflected Cherenkov light. Background from electromagnetic showers caused by radiative Bhabha collisions is proportional to luminosity. Its effect will become more important in time as luminosity increases.

$B A B A R$ is equipped with radiation monitors which can limit beam injections or dump the beam if radiation rates become too severe. So far the accumulated radiation dose is within tolerance for all detector subsystems. 


\subsubsection{Performance}

As of July 2003 PEP-II had delivered an integrated luminosity of $136.93 \mathrm{fb}^{-1}$, of which $130.65 \mathrm{fb}^{-1}$ had been recorded by the BABAR detector (see Fig. 2.2). The recorded data are divided into 3 running periods, Run 1-3. Run 1 consists of $23 \mathrm{fb}^{-1}$ of data taken between October 1999 and October 2000. Run 2 started in February 2001 and ended in June 2002 and a total integrated luminosity of $59 \mathrm{fb}^{-1}$ was recorded. Run 3 ran from December 2002 to July 2003, taking $34 \mathrm{fb}^{-1}$ of data. PEP-II has been running remarkably well, exceeding almost all design expectations and goals.

\subsection{The BABAR Detector}

The BABAR detector (Fig. 2.3) has been developed to maximise the physics potential of the PEP-II asymmetric $B$ Factory. It is made up of 5 separate subsystems immersed in a $1.5 \mathrm{~T}$ magnetic field. Closest to the IP is the silicon vertex tracker (SVT) which provides precise measurements of decay vertices. This is followed by the drift chamber $(\mathrm{DCH})$. This in turn is surrounded by a detector of internally reflected Cherenkov light (DIRC) which provides particle identification. Next comes a CsI crystal totally-absorbing electromagnetic calorimeter (EMC), and finally an instrumented flux return (IFR) for muon and neutral hadron identification. These systems, together with the trigger, are discussed in more detail in the following sections.

The right-hand coordinate system used in BABAR is defined with the z-axis coinciding with the principle axis of the drift chamber. The positive y-axis points upward and the positive $\mathrm{x}$-axis points away from the centre of the PEP-II storage ring. The angles $\theta$ and $\phi$ are defined as usual. 


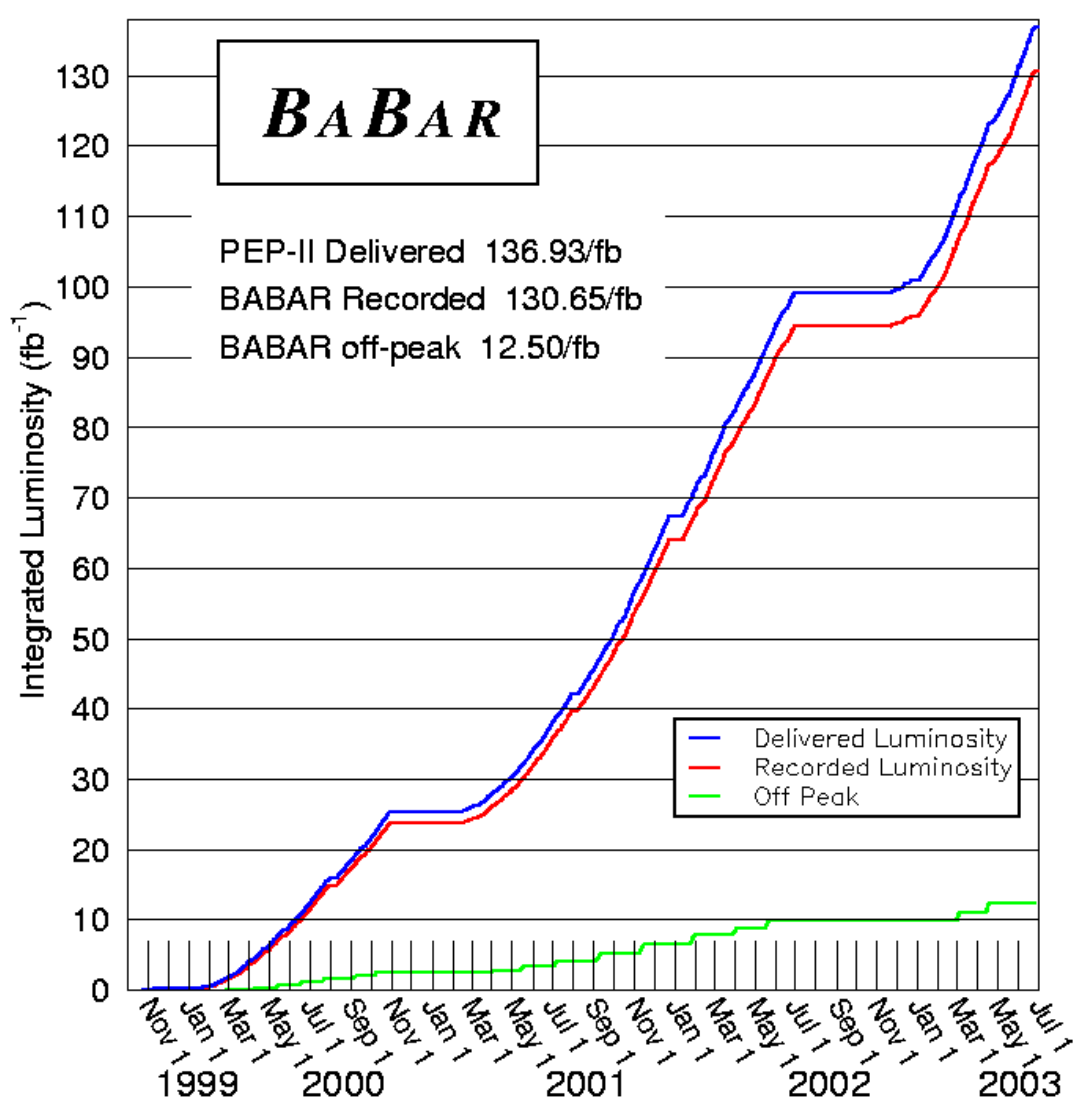

Figure 2.2: Luminosity delivered by PEP-II and recorded by $B A B A R$.

\subsection{The Silicon Vertex Tracker (SVT)}

\subsubsection{Requirements}

Together with the DCH, the SVT provides the tracking for the BABAR detector. The main role of the SVT is to provide precision vertexing of decays which is required to measure time-dependent $C P$ asymmetries. At the PEP-II boost $(\beta \gamma=0.56)$, the mean separation along the z-axis of the two $B$ meson decay vertices is $250 \mu \mathrm{m}$. In order not to significantly impact the resolution on the measured $C P$ asymmetry, 


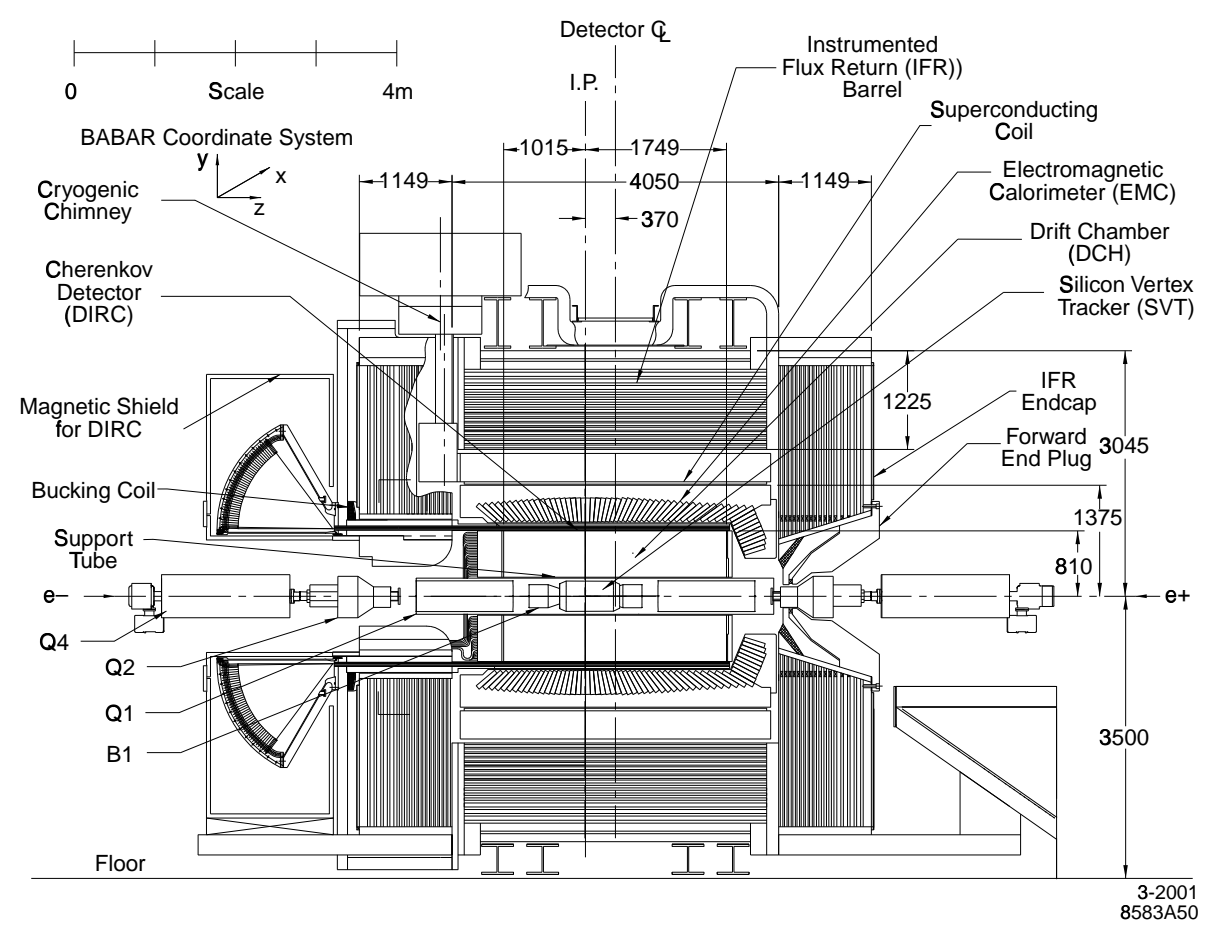

Figure 2.3: Cross section of the $B A B A R$ detector.

the resolution on the $B$ vertex separation is required to be approximately half the average separation between the decay vertices, meaning the SVT is required to provide a resolution on a single $B$ decay vertex of less than $80 \mu \mathrm{m}$. In addition, some decays of interest produce low transverse momentum $\left(p_{T}\right)$ tracks (e.g., slow pions produced in $D^{*} \rightarrow D^{0} \pi$ decays have $p_{T}$ in the range 50-120 MeV/c) which may not be reconstructed in the $\mathrm{DCH}\left(p_{T}>120 \mathrm{MeV} / c\right.$ is required for reliable reconstruction in the $\mathrm{DCH}$ ). The SVT is required to perform standalone tracking for these tracks. The SVT is also crucial to the performance of the DIRC. It provides the best measure of track angles which are required to correctly resolve the Cherenkov angle measured in the DIRC.

\subsubsection{Design}

The SVT comprises five layers of double-sided silicon microstrip detectors. The layers are composed of $6,6,6,16$ and 18 modules respectively. In order to minimise the amount of silicon needed to cover the required solid angle and to increase the crossing angle for particles close to the edge of acceptance, the outer layers are arch 
shaped. To ensure full azimuthal coverage, and to aid in alignment, the modules in the inner three layers are forced to overlap by tilting them at an angle of $5^{\circ}$ in $\phi$. This tilting is not possible in the outer layers where overlap is achieved by stacking the modules in each layer into two sub-layers. The layout is shown in Fig. 2.4 and Fig. 2.5. Each layer is electronically separated into a forward and backward half module. In total the SVT contains $0.96 \mathrm{~m}^{2}$ of active silicon and has a $90 \%$ solid angle acceptance in the centre of mass $(\mathrm{CM})$ frame.

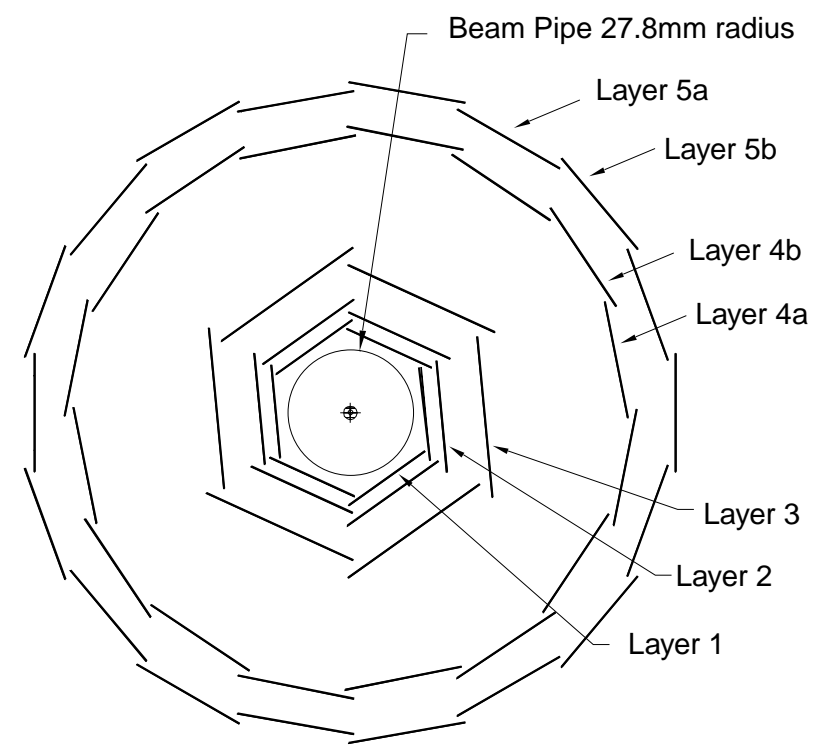

Figure 2.4: Schematic of SVT silicon layers in the $x-y$ plane.

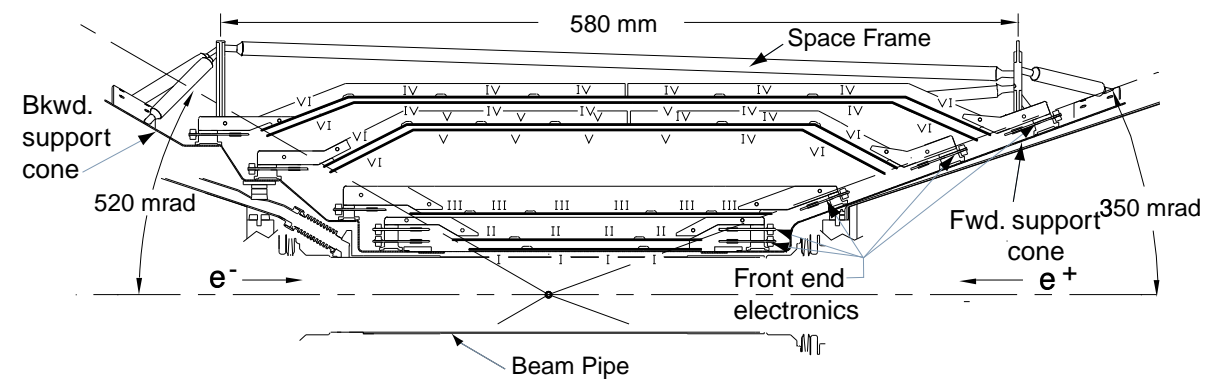

Figure 2.5: Schematic of SVT silicon layers in the z plane.

One side of the $300 \mu \mathrm{m}$ thick silicon wafer has $\mathrm{p}^{+}$types strips, and the other $\mathrm{n}^{+}$type (interspersed with p type strips to provide an inter-strip resistance $>100 \mathrm{M} \Omega$ ). The strips on either side of the silicon are orthogonal; the outer strips are orientated along 
the beam line (to measure the $\phi$ coordinate) and the inner strips are perpendicular to the beam line (to measure the $\mathrm{z}$ position). Due to Coulomb scattering, the most important measurements of the track parameters are made as close to the beam pipe as possible. The track impact parameter measurements are made by the first 3 layers, which require a spatial resolution of 10-15 $\mu \mathrm{m}$ in the perpendicular direction. The outer layers are required to match tracks to further tracking in the $\mathrm{DCH}$ and for stand-alone tracking of low $p_{T}$ tracks. They require a resolution of about $40 \mu \mathrm{m}$. The inner 3 layers have a readout pitch of $100 \mu \mathrm{m}$ in the $\mathrm{z}$ direction, the outer layers have a pitch of $210 \mu \mathrm{m}$. The pitch of the readout strips in the $\phi$ direction varies from $50 \mu \mathrm{m}$ to $210 \mu \mathrm{m}$. In the inner 3 layers each $\mathrm{z}$ strip is read out by one electronics channel. In layers 4 and 5 the number of strips is greater than the available channels, so two strips are "ganged" into one channel. The resulting ambiguity is resolved in pattern recognition. The $\phi$ strips in adjacent half modules are bonded together to form one readout channel. The number of readout channels is reduced by introducing a floating channel (not instrumented) between most readout channels. The SVT has a total of 150,000 readout channels.

The readout electronics convert the signals from the microstrips into Time Over Threshold (TOT) measurements which are a quasi-logarithmic function of the collected charge. The signal-to-noise ratio is greater than 15 for minimum ionising particles in all modules. A hit in the SVT corresponds to a deposited charge greater than $0.95 \mathrm{fC}$. The output is sampled at $30 \mathrm{MHz}$ and stored in a 193 deep buffer. Upon receipt of a L1 trigger the time and TOT are retrieved and stored in a four event buffer. It is shipped from here upon receipt of a L1 trigger accept. The radiation hardness of the electronics have been studied up to $2.4 \mathrm{MRad}$ using a ${ }^{60} \mathrm{Co}$ source. The gain decreased by $20 \%$ and the noise increased by less than $15 \%$.

\subsubsection{Performance}

Under normal operation the SVT has an occupancy of $3 \%$ in the inner layers and less than $1 \%$ in the outer layers. This is obviously sensitive to beam-induced backgrounds and significant azimuthal variation is observed. The first step in signal 
reconstruction is to reduce the number of channels by removing out of time hits, defined as hits outside of a 200ns window (the relevant time is defined by the DCH). The charge deposited per strip is calculated from the TOT value and clusters are formed by grouping adjacent strips with consistent times. The position of the cluster is then calculated using

$$
x=\frac{\left(x_{1}+x_{i}\right)}{2}+\frac{p}{2} \frac{\left(Q_{i}-Q_{1}\right)}{\left(Q_{i}+Q_{1}\right)}
$$

where $x_{i}$ is the position of the i-th strip, $Q_{i}$ is the collected charge and $\mathrm{p}$ is the readout pitch. The position resolutions achieved are shown in Fig. 2.6.
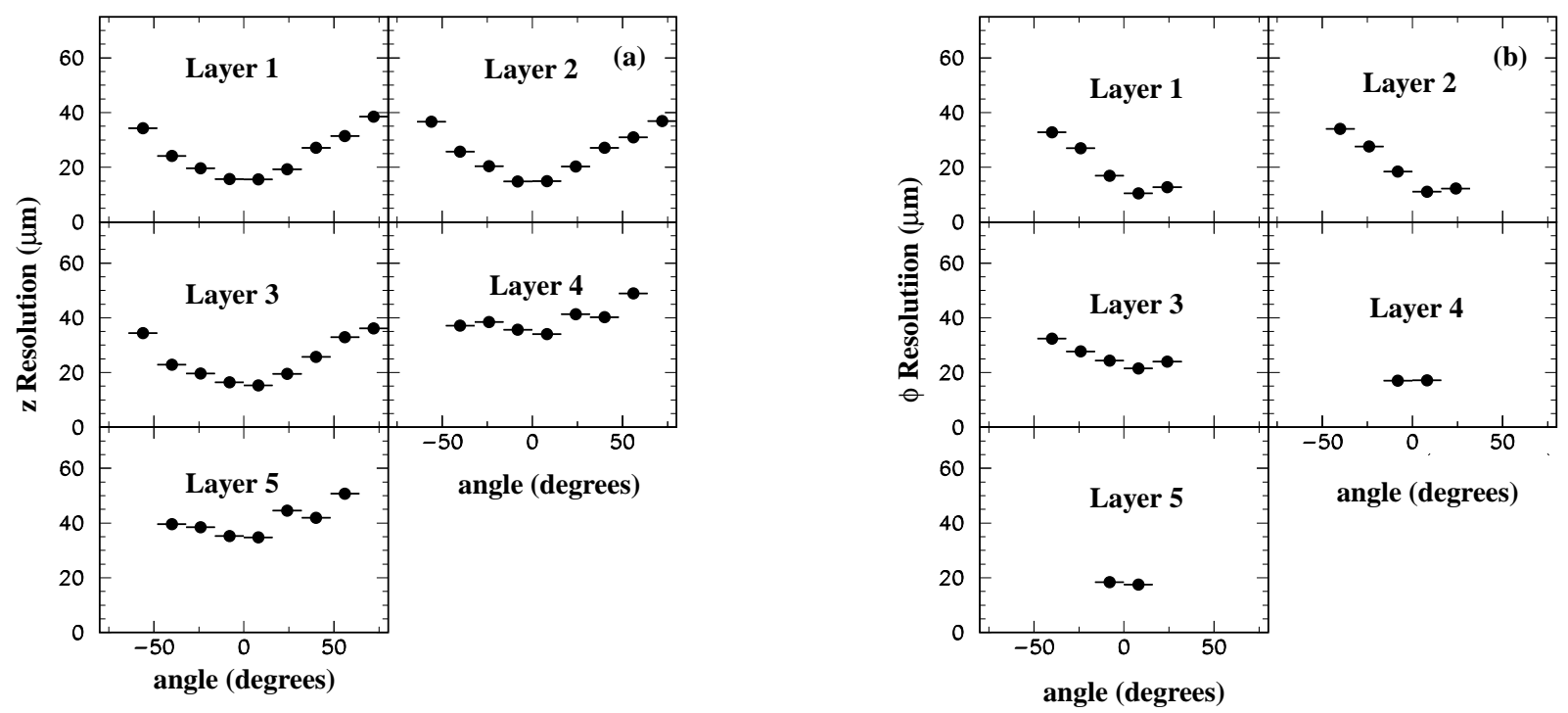

Figure 2.6: The resolution of hits in the SVT for the a) z and b) $\phi$ direction as a function of incidence angle. Each plot shows a different layer.

The efficiency of the SVT is measured by comparing the number of associated hits to the number of tracks crossing the active area using $\mu^{+} \mu^{-}$events. During installation 9 of 208 readout sections (each section corresponds to one side of a half module) were damaged and are currently not working. Excluding these modules, the SVT has a measured efficiency of $97 \%$.

The SVT also provides $\mathrm{d} E / \mathrm{d} x$ measurements by summing the deposited charge over microstrips in a cluster. Up to 10 measures can be made per track, one for each side of a layer. A kaon/pion separation of $2 \sigma$ can be achieved up to a momentum of $500 \mathrm{MeV} / c$. 
Information from the SVT and DCH is combined for track reconstruction. Discussion of their combined performance is left until after the DCH section.

\subsection{The Drift Chamber (DCH)}

\subsubsection{Requirements}

The DCH is required to detect, and measure the momentum of, charged particles with high efficiency. These measurements, along with those made by the SVT, allow exclusive $B$ and $D$ decays to be reconstructed with low background. The DCH is the only source of tracking for particles that decay outside the volume of the SVT e.g. $K_{S}^{0} \rightarrow \pi^{+} \pi^{-}$. This leads to the requirement of a longitudinal resolution of $1 \mathrm{~mm}$. For low momentum tracks the DCH measurements dominate the error on the extrapolation of tracks to further BABAR subsystems.

The DCH provides particle identification through $\mathrm{d} E / \mathrm{d} x$ measurements. This is particularly important for low momentum tracks where the DIRC is ineffective. A resolution of $7 \%$ allows $\pi / \mathrm{K}$ separation up to $700 \mathrm{MeV} / c$.

All of this needs to be achieved in a high beam background environment (rates of $5 \mathrm{kHz}$ in the innermost cells) and with the ability to supply information to the charged particle trigger with a maximum time jitter of $0.5 \mu \mathrm{s}$. To reduce errors on track measurements from Coulomb scattering, the amount of material before the DCH needs to be kept to a minimum.

\subsubsection{Design}

The DCH, shown in Fig. 2.7, has an outer radius of $81 \mathrm{~cm}$ and is just under $3 \mathrm{~m}$ long. It is divided into 40 layers of hexagonal cells (7,104 cells in total) which provide up to 40 measurements for particles with $p_{T}>180 \mathrm{MeV} / c$. Each cell is composed of a sense wire surrounded by six field wires. To account for the boost the centre of the 
$\mathrm{DCH}$ is offset in $\mathrm{z}$ from the interaction point, meaning the particles traverse at least half the layers of the $\mathrm{DCH}$ down to $\theta=17.2^{\circ}\left(152.6^{\circ}\right)$ in the forward (backward) direction.

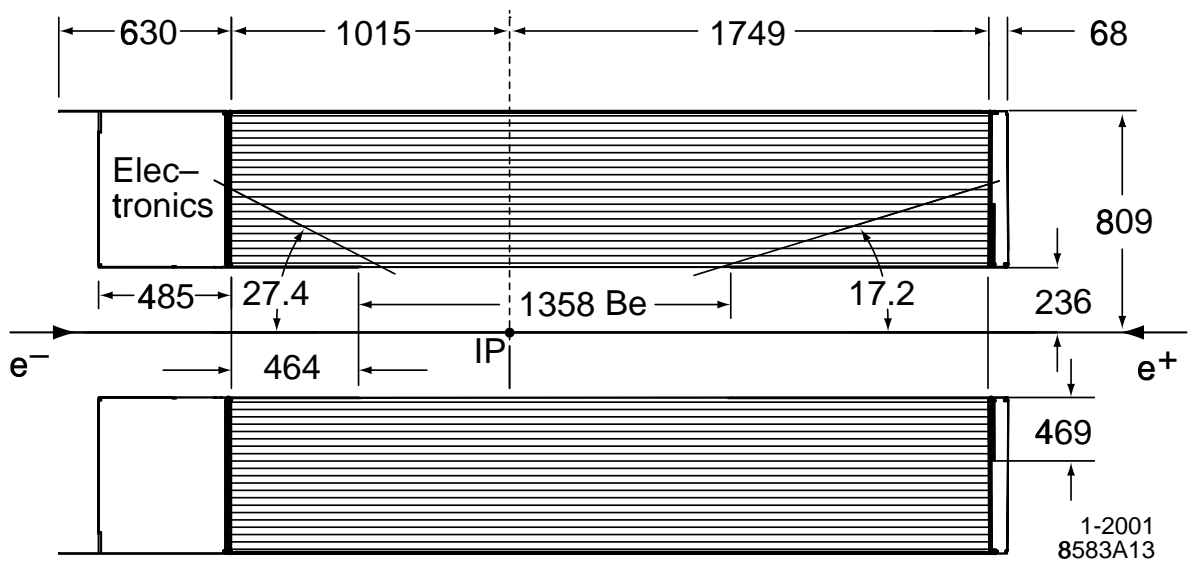

Figure 2.7: Cross section of the DCH.

The 40 layers of cells are further divided into 10 superlayers of 4 layers each. Longitudinal position measurements are provided by arranging the sense wires within each superlayer into either axial or stereo orientations. The stereo angles vary between $\pm 45 \mathrm{mrad}$ and $\pm 76 \mathrm{mrad}$. Fig. 2.8 shows the configuration of the first 4 super-layers. The design operating voltage for the sense wires is $1960 \mathrm{~V}$. The voltage was dropped to $1900 \mathrm{~V}$ midway through the first Run 1 because of concern for a small area which sustained damage during commissioning. Since the start of Run 2 the DCH has been operating at $1930 \mathrm{~V}$. The DCH is filled with a 80:20 mixture of helium:isobutane gas which represents less than $0.2 \% X_{0}$ of material. The inner wall represents a further $0.28 \% X_{0}$ at normal incidence.

All of the readout electronics and high voltage distributors are positioned at the backward endplate of the DCH. The electronics provide both timing information for drift time measurements and total charge collection readings for $\mathrm{d} E / \mathrm{d} x$ information. The electronics are calibrated daily to determine channel-by-channel constants and thresholds. 


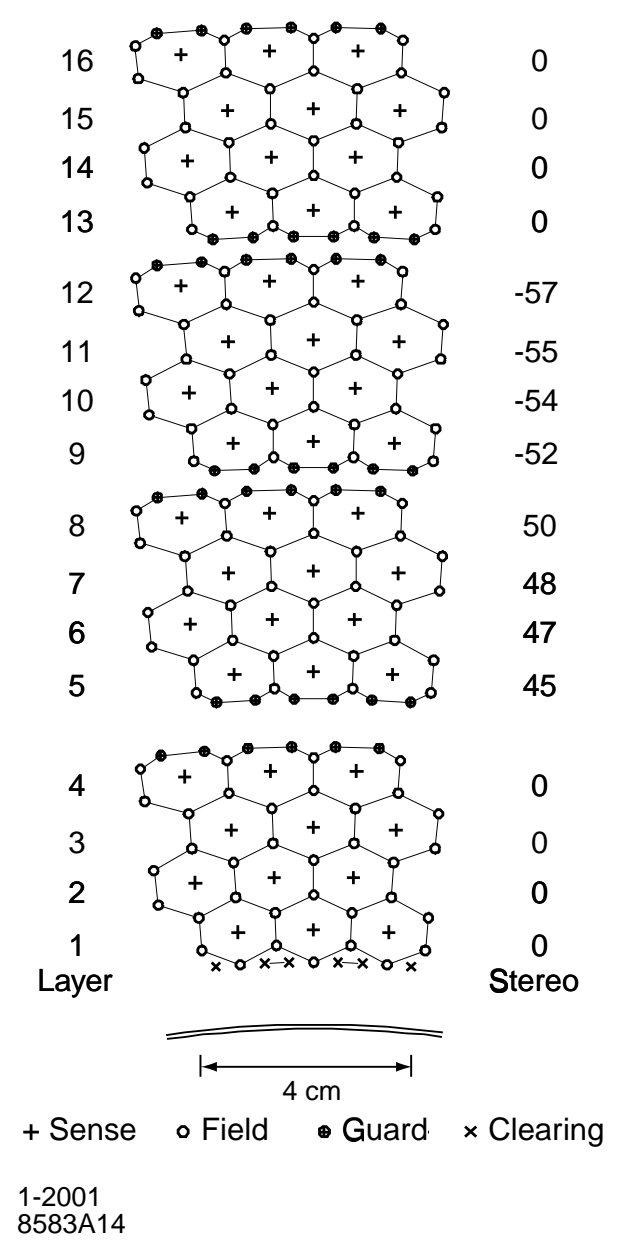

Figure 2.8: First four superlayers of the DCH.

\subsubsection{Performance}

Fig. 2.9 shows $\mathrm{d} E / \mathrm{d} x$ measurements taken by the $\mathrm{DCH}$, overlaid with the BetheBloch predictions for different particle types derived from control samples. The final $\mathrm{d} E / \mathrm{d} x$ is determine by averaging the individual cell measurements and correcting for gain variations, pedestal subtractions, changes in gas conditions, cell geometry and other effects. The resolution on $\mathrm{d} E / \mathrm{d} x$ has been measured using a sample of $e^{ \pm}$ from Bhabha events with the DCH operating at a voltage of $1900 \mathrm{~V}$. The resolution is determined to be $7.5 \%$, close to the design value of $7 \%$.

The drift time measurements are used to determine the position of the track in each cell. The time-to-distance relations are determined from $e^{+} e^{-}$and $\mu^{+} \mu^{-}$events. The DCH is capable of a position resolution of $140 \mu \mathrm{m}$ averaged over the cell. 


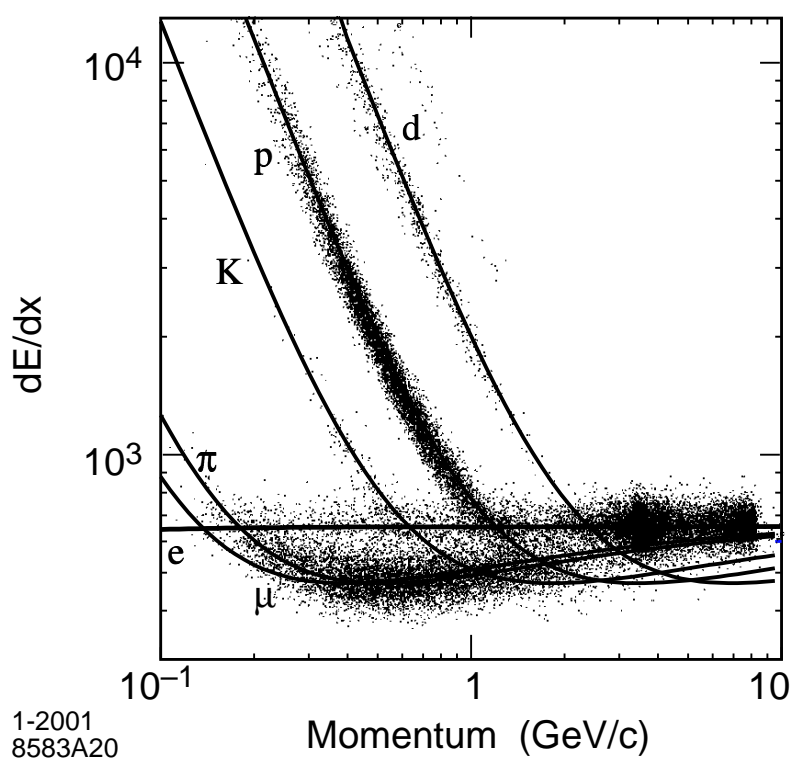

Figure 2.9: $\mathrm{d} E / \mathrm{d} x$ of particles measured in the DCH. The solid lines show the Bethe-Bloch predictions derived from control samples. The y axis is in arbitrary units.

The tracking efficiency of the DCH is measured by comparing the number of tracks reconstructed in the $\mathrm{DCH}$ to the number of tracks reconstructed in the SVT (provided they fall in the $\mathrm{DCH}$ acceptance). The results are shown in Fig. 2.10. The average efficiency at $1960 \mathrm{~V}$ is $98 \pm 1 \%$ for tracks above $200 \mathrm{MeV} / c$.

\subsubsection{Combined SVT and DCH Tracking Performance}

The BABAR tracking combines information from both the SVT and DCH. Charged tracks are defined in terms of 5 parameters $\left(d_{0}, \phi_{0}, \omega, z_{0}, \tan \lambda\right)$, which are measured at the closest approach to the $\mathrm{z}$ axis. $d_{0}$ and $z_{0}$ are the distances of closest approach to the origin. The angle $\phi_{0}$ is the azimuth of the track at the position of closest approach, $\lambda$ is the dip angle relative to the transverse plane and $\omega=1 / p_{T}$. The sign of $d_{0}$ and $\omega$ depends upon the charge of the track.

Tracks are reconstructed by first attempting to make tracks in the $\mathrm{DCH}$ by joining segments of DCH hits together. Once a candidate track is found in the DCH it is extrapolated into the SVT and any appropriate SVT segments are added. A fit is then performed on the full set of DCH and SVT hits. A secondary algorithm finds 

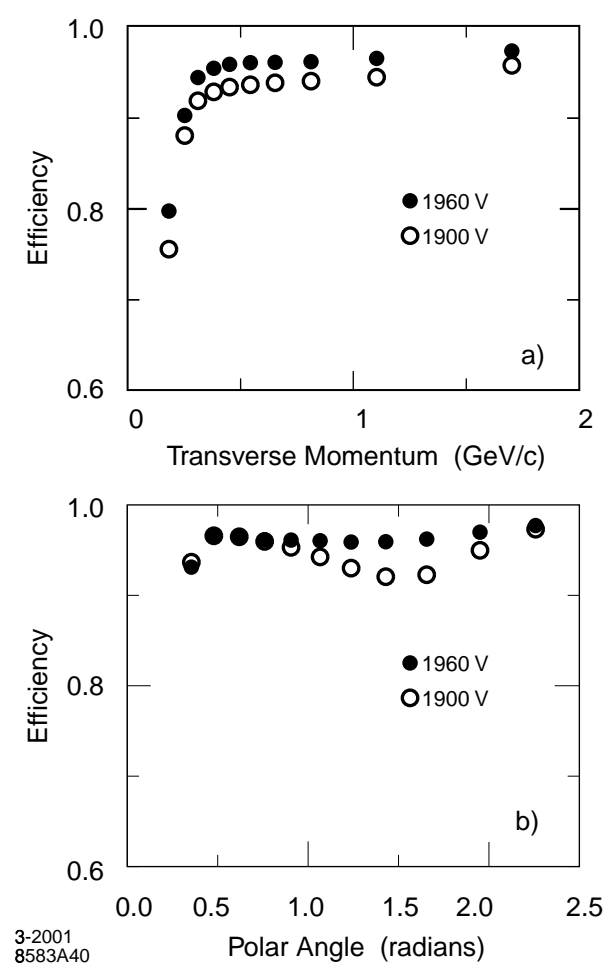

Figure 2.10: The plots shows the ratio of tracks reconstructed in the DCH compared to tracks reconstructed in the SVT (confined to the DCH acceptance). The filled circles are with the DCH operating at $1960 \mathrm{~V}$, the open circles at $1900 \mathrm{~V}$.

SVT-only tracks.

The resolution on these track parameters can be measured using cosmic ray data. The cosmic rays transverse both halves of the detector. By plotting the difference in the parameters for the track reconstruced in each half we can gain a measure of the resolutions. Such distributions are plotted in Fig. 2.11 for $p_{T}>3 \mathrm{GeV} / c$. The resolution of the parameters are $\sigma_{d_{0}}=23 \mu \mathrm{m}, \sigma_{\phi_{0}}=0.43 \mathrm{mrad}, \sigma_{\mathrm{z}_{0}}=29 \mu \mathrm{m}$ and $\sigma_{\operatorname{tan\lambda }}=0.53 \times 10^{-3}$.

The resolution on $p_{T}$ can also be measured using cosmic rays, the results are presented in Fig. 2.12. A fit to this graph gives $\sigma_{p_{T}} / p_{T}=(0.13 \pm 0.01) \% \times p_{T}+(0.45 \pm$ $0.03) \%$. The resolution on $p_{T}$ is dominated by the $\mathrm{DCH}$.

The SVT and DCH have been performing close to expectations from the start of operations. 


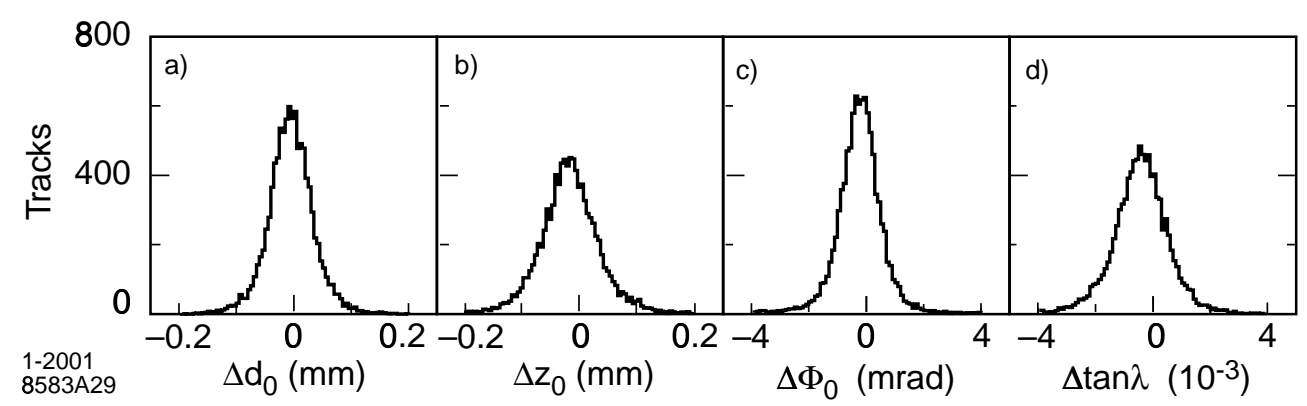

Figure 2.11: Resolution of track parameters measured by taking the difference of the two halves of the tracks fitted to cosmic ray muons. The tracks have $p_{T}>3 \mathrm{GeV} / c$.

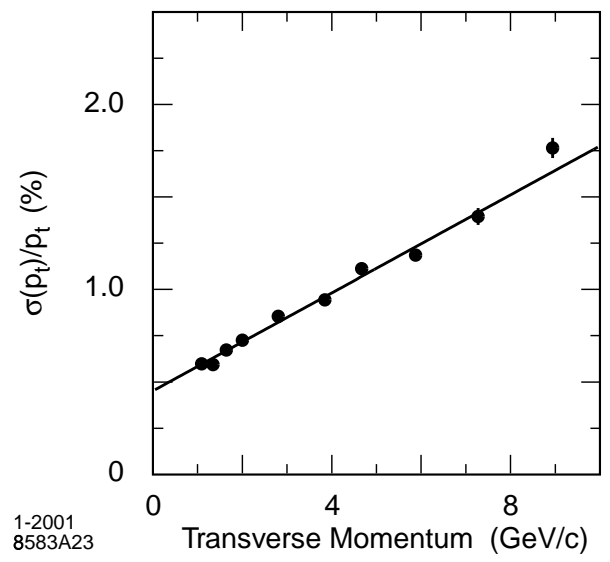

Figure 2.12: Resolution of $p_{T}$ from tracks with both DCH and SVT hits, measured using cosmic ray data.

\subsection{The Detector of Internally Reflected Cherenkov Light (DIRC)}

\subsubsection{Requirements}

Along with the DCH, the DIRC provides Particle Identification (PID) for BABAR. This is important for flavour tagging in neutral $B$ decays via the cascade $b \rightarrow c \rightarrow s$, where the momentum of the kaons can extend up to $2 \mathrm{GeV} / c$, and for kaon/pion separation for rare decays such as $B^{0} \rightarrow \pi^{+} \pi^{-}$and $B^{0} \rightarrow K^{+} \pi^{-}$, where the momentum of the particles is in the range $1.7-4.2 \mathrm{GeV} / c$.

The DIRC was expected to provide $\pi / K$ separation of $4 \sigma$ and above for all tracks from $B$ meson decays with momentum above the pion Cherenkov threshold. The 
DIRC loses separation power when the particle momenta dip below $700 \mathrm{MeV}$. In this range, PID is primarily provided by $\mathrm{d} E / \mathrm{d} x$ measurements in the $\mathrm{DCH}$.

The DIRC is required to be thin and uniform in terms of radiation lengths so as not to adversely affect calorimetry energy resolution. It is also desirable to keep the DIRC thin spatially in order to keep the volume small and hence minimise the cost of the calorimetry. Finally the DIRC needs to be fast enough and sufficiently radiation hard to operate in the high luminosity BABAR environment.

\subsubsection{Design}

The basic principle of the DIRC is that of Cherenkov radiation. When particles move through a material at a speed greater than the speed of light in that material they emit radiation at a characteristic angle to the direction of the track $\left(\theta_{c}\right) . \theta_{c}$ is given by $\cos \theta_{c}=1 / n \beta$, where $n$ is the refractive index of the material, $\beta=v / c, v$ is the speed of the particle and $c$ is the speed of light. The material used in the DIRC is fused silica which has $n=1.473$.

A schematic of the DIRC is shown in Fig. 2.13. The DIRC is made up of a 12-sided polygon barrel, each side being one bar box. These bar boxes contain 12 fused silica bars that are optically isolated from each other by a $150 \mu \mathrm{m}$ air gap and custom made sheaths of aluminium foil. The individual silica bars are $17 \mathrm{~mm}$ thick, $35 \mathrm{~mm}$ wide and $1.225 \mathrm{~m}$ long. Four bars are glued together to give a total length of $4.9 \mathrm{~m}$. The Cherenkov photons produced are total internally refracted down the silica bar and collected by photomultiplier tubes (PMT) at the back end of the detector (a mirror is placed at the forward end of the bars). There are 10752 of these PMTs housed in a conic standoff box which is filled with 6,000 litres of purified water. Water is a suitable choice of material as it has both a refractive index $(n=1.346)$ close to that of the silica (so total internal refraction at the silica-water interface is minimised) and also a similar chromaticity index (dispersion is minimised).

The photons are focused on the PMTs by the pin-hole defined by the silica bars. The angles $\alpha_{x}, \alpha_{x}$ and $\alpha_{z}$ are defined by the vector from the PMT to the bar which 


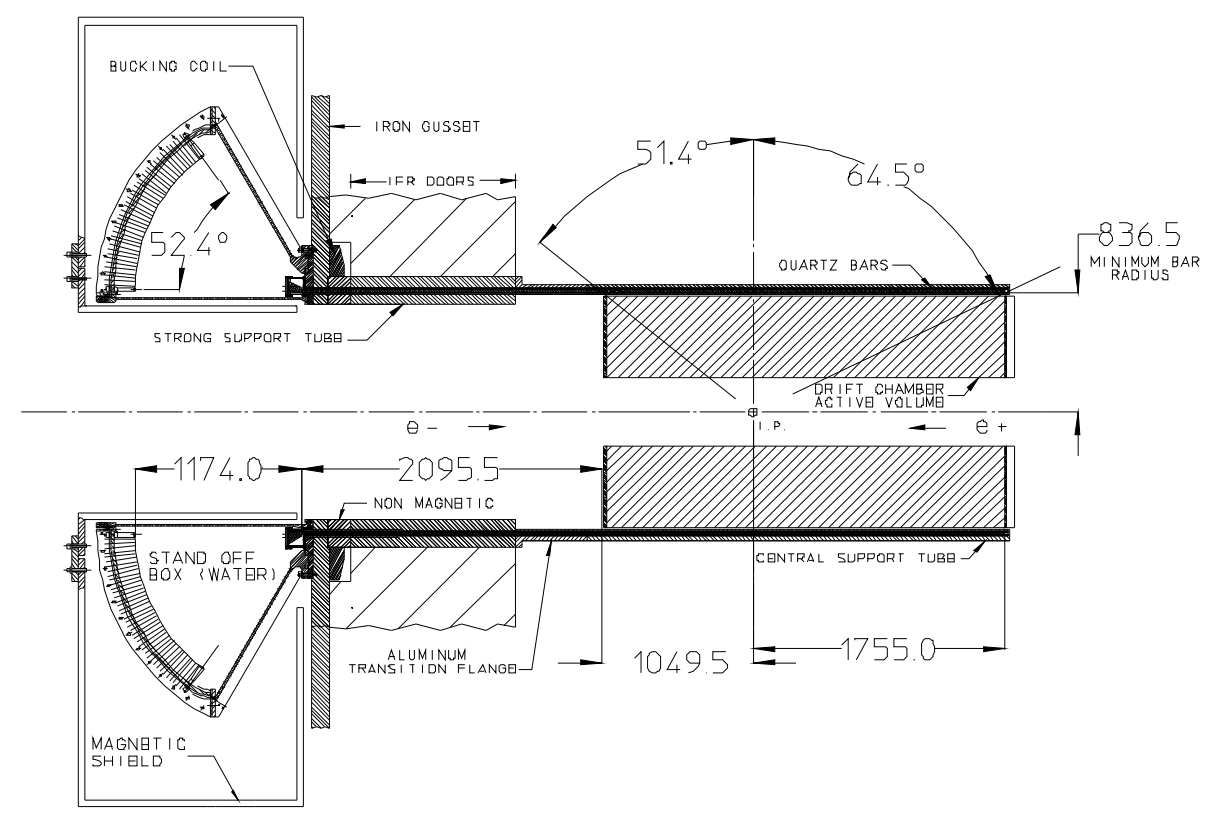

Figure 2.13: Cross section of the DIRC.

the particle traversed (the direction and angle of the track is known from prior tracking). These angles are used to determine $\theta_{c}$ and $\phi_{c}$ (the azimuthal angle of the Cherenkov photon to the track). Timing information is used to reduce the number of observed background photons as illustrated in Fig. 2.14.
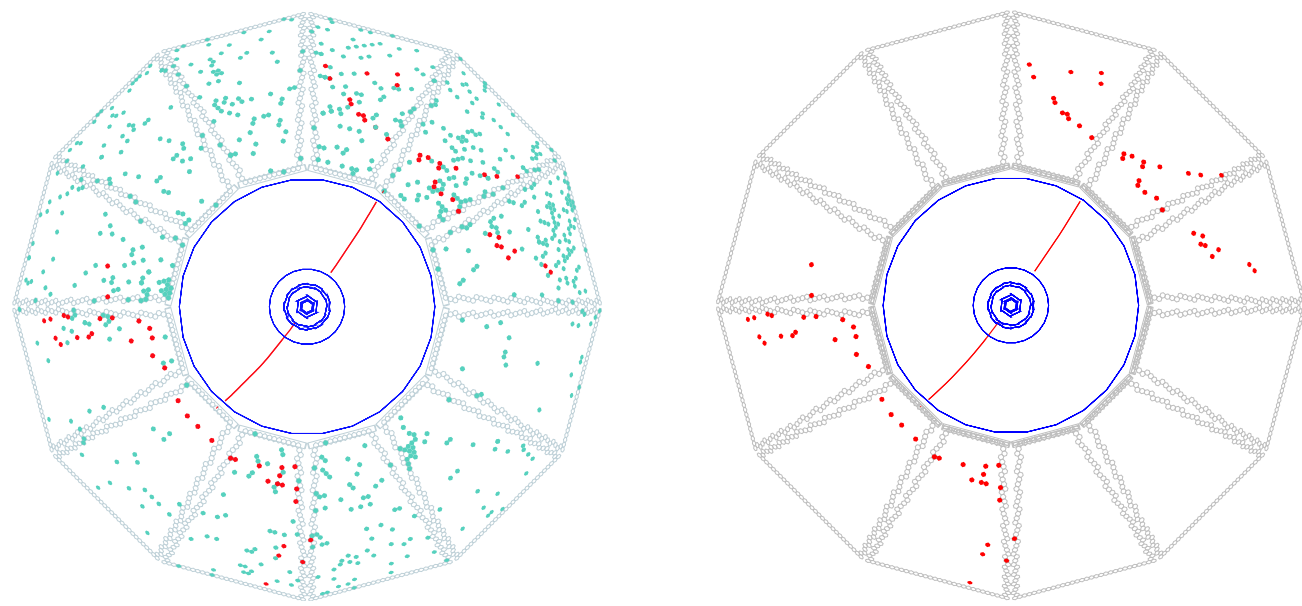

Figure 2.14: $e^{+} e^{-} \rightarrow \mu^{+} \mu^{-}$event reconstructed using two different time cuts. The left shows hits within $\pm 300 \mathrm{~ns}$, on the right the time window has been reduced to $\pm 8 \mathrm{~ns}$ of the expected arrival time.

The arrangement of the PMTs leads to an angular resolution of $7 \mathrm{mrad}$, slightly larger than the rms spread of the intrinsic photon production and transmission dispersion. The overall single photon resolution is estimated to be about $10 \mathrm{mrad}$. 
The timing of the photon arrival at the PMTs is calibrated using flashing blue LEDs and real collision data.

\subsubsection{Performance}

The resolution on the track Cherenkov angle $\left(\sigma_{c, t r a c k}\right)$ should scale as

$$
\sigma_{c, t r a c k}=\frac{\sigma_{c, \gamma}}{\sqrt{N_{p e}}}
$$

where $\sigma_{c, \gamma}$ is the single photon Cherenkov angle resolution and $N_{p e}$ is the number of photons. The average number of photons varies with the angle of incidence of the track, from 20 for small angles to 65 at large polar angles. Fig. 2.15 shows $\sigma_{c, \gamma}$ (around $10.2 \mathrm{mrad}$ ) and the expected arrival time spread (1.5ns). $\sigma_{c, t r a c k}$ is measured to be $2.5 \mathrm{mrad}$ using $\mu^{+} \mu^{-}$events (Fig 2.16).
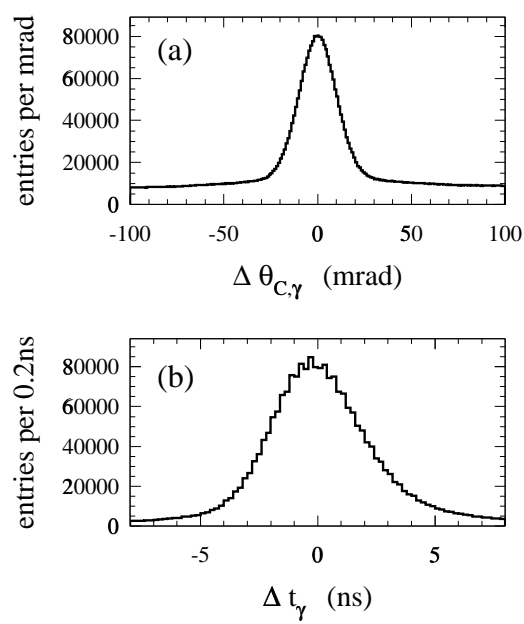

Figure 2.15: The difference between (a) the measured and expected Cherenkov angle for single photons $\Delta \theta_{c, \gamma}$ and (b) the measured and expected photon arrival time for $\mu^{+} \mu^{-}$events.

The measured kaon/pion separation at $4 \mathrm{GeV} / c$ is around $2 \sigma$. The mean kaon selection efficiency measured using $D^{0} \rightarrow K^{-} \pi^{+}$events is $96.2 \pm 0.2 \%$ (statistical error) with a pion misidentification of $2.1 \pm 0.1 \%$. 


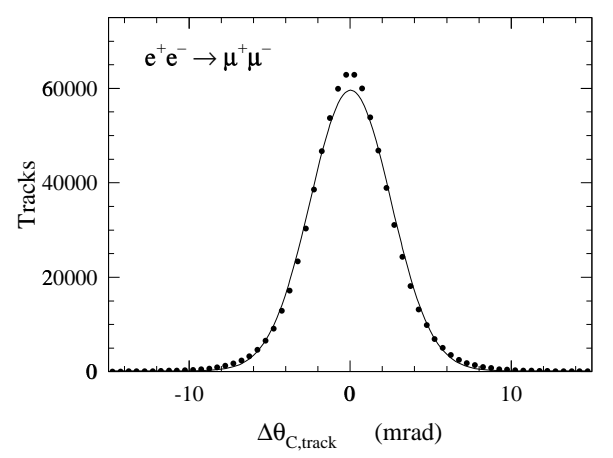

Figure 2.16: The difference between measured and expected Cherenkov angle $\left(\Delta \Theta_{c, \text { track }}\right)$ for $\mu^{+} \mu^{-}$events.

\subsection{The Electromagnetic Calorimeter (EMC)}

\subsubsection{Requirements}

The EMC is required to measure electromagnetic showers ranging from $20 \mathrm{MeV}$ to $9 \mathrm{GeV}$. The lower bound is determined by the need to measure $\pi^{0}$ and $\eta$ in high multiplicity $B$ meson decays, the upper by the need to measure processes such as $e^{+} e^{-} \rightarrow e^{+} e^{-}, e^{+} e^{-} \rightarrow e^{+} e^{-} \gamma$ and $e^{+} e^{-} \rightarrow \gamma \gamma$ for luminosity measurements and calibrating purposes. As well as contributing to flavour tagging in neutral $B$ decays, the EMC is of value in any semileptonic processes as it provides electron PID. $\pi^{0}$ reconstruction in rare decays such as $B \rightarrow \pi^{0} \pi^{0}$ requires an energy resolution of $1-2 \%$ for photons. Above $2 \mathrm{GeV}$ the resolution on the $\pi^{0}$ mass is dominated by angular resolution, which is required to be at the level of a few mrad. The EMC needs to operate in a $1.5 \mathrm{~T}$ magnetic field and high radiation environment for the lifetime of the experiment.

\subsubsection{Design}

The BABAR EMC is a hermetic (90\% solid angle coverage in the CM frame) totalabsorption calorimeter composed of finely segmented CsI crystals. The energy resolution of a homogeneous crystal calorimeter is described empirically by two terms 
which sum in quadrature

$$
\frac{\sigma_{E}}{E}=\frac{a}{\sqrt[4]{E(\mathrm{GeV})}} \oplus b
$$

where $E$ is the energy of the photon. The $a$ term describes photon statistics, electronics noise and beam-generated background. This term is dominant at low energies. The $b$ term is due to non-linearities in light collection, leakage and absorption before the calorimeter and calibration uncertainties. This term dominates for energies above $1 \mathrm{GeV}$. Under ideal conditions values of $a$ and $b$ of around 1-2\% may be possible, but very difficult to achieve. The angular resolution is also described by an empirical relation

$$
\sigma_{\theta}=\sigma_{\phi}=\frac{c}{\sqrt{E(\mathrm{GeV})}} \oplus d .
$$

The design goals were $c \approx 3 \mathrm{mrad}$ and $d \approx 1 \mathrm{mrad}$.

Fig. 2.17 shows a schematic of the EMC. It consists of a cylindrical barrel section, which extends down to $38.2^{\circ}$ in the backward direction, and a forward endcap which extends to $15.8^{\circ}$ in the forward direction. The barrel contains 5760 crystals arranged in 48 rings with 120 identical crystals in each. The endcap holds 820 crystals in eight rings, the innermost two rings (those at the smallest polar angle, $15.8^{\circ}$, and closest to the beam pipe) of which just serve to contain the full lateral showers of the outer rings. The barrel and outer 5 rings of the endcap has less than $0.3-0.6 X_{0}$ of material between them and the IP. The inner rings of the endcap have the SVT support structure in front of then and so have up to $3.0 X_{0}$.

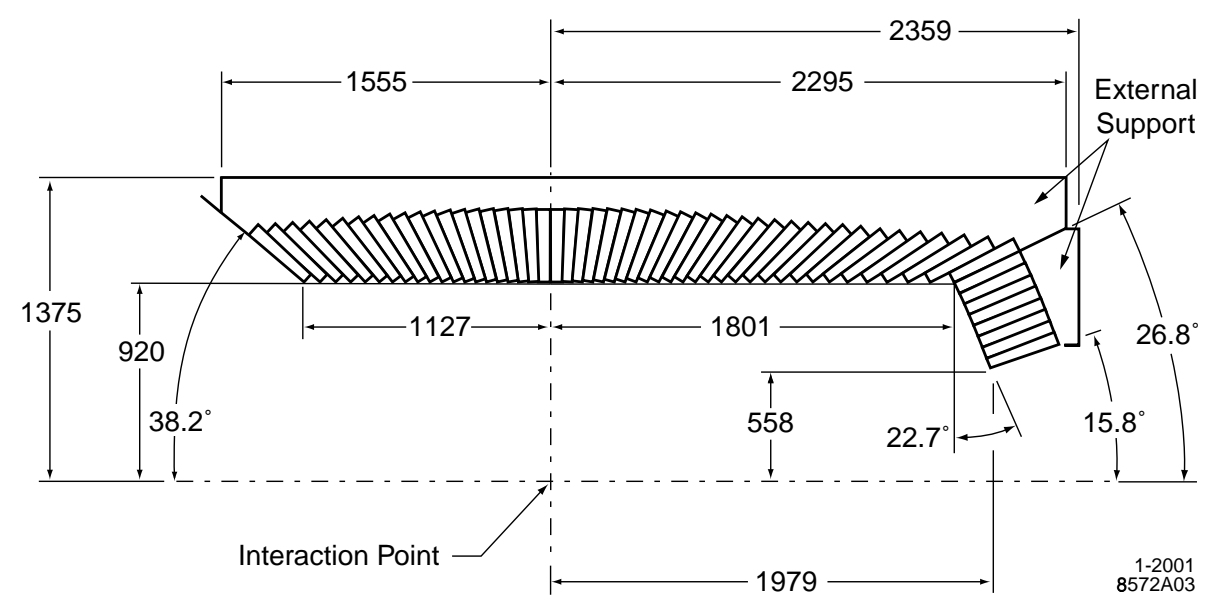

Figure 2.17: Cross section of the top half of the EMC. 
Thallium-doped CsI crystals were chosen for the EMC. They have a radiation length of $1.85 \mathrm{~cm}$, a Molière radius of $3.8 \mathrm{~cm}$ and a light yield of 50,000 photons $/ \mathrm{MeV}$. The small Molière radius allows for good angular resolution. The dimensions of the crystal differ with position but they typically have a front face area of $4.7 \times$ $4.7 \mathrm{~cm}^{2}$. The small radiation length allows for the design of a compact calorimeter; the crystals range from $29.6 \mathrm{~cm}$ in the backward direction to $32.4 \mathrm{~cm}$ in the forward direction. The high light yield means silicon photodiodes can be used to measure the light output of the crystals. These operate well in high magnetic fields. To give redundancy, each crystal has two photodiodes attached. The crystals act as a light guide to collect scintillation light. Any light which is not internally reflected at the polished surface is recovered by two layers of $165 \mu \mathrm{m}$ white reflector. The crystals are further wrapped in aluminium foil to provide a Faraday shield and a layer of mylar to assure electrical isolation. The light yield uniformity of the crystals was tested using a radiation source before the crystals were placed in the EMC. A tolerance of $\pm 2 \%$ was required in the front half of the crystals and $\pm 5 \%$ at the rear face. The crystals and endcap electronics are cooled by Fluorinert and the barrel electronics by water. It is important to maintain the system at a constant temperature, especially as the leakage current from the photodiodes rises exponentially with temperature.

The electronics for the EMC need to have negligible impact on the energy resolution in an energy range from $20 \mathrm{MeV}$ to $9 \mathrm{GeV}$. This is achieved by amplifying the output from the photodiodes into four gain ranges, $\times 1, \times 4, \times 32$ and $\times 256$. In terms of energy these ranges correspond to $0-50 \mathrm{MeV}$ (the least significant bit corresponds to $50 \mathrm{keV}$ ), $50-400 \mathrm{MeV}, 0.4-3.2 \mathrm{GeV}$ and $3.2-13.0 \mathrm{GeV}$. The electronics are calibrated by using a charge injection system. The pedestals of the preamps are measured by taking random triggers with no beams.

During the first years of operation there were a number of non-linearities discovered in the response of the EMC electronics, some of which have been resolved. These included non-linearities of up to $12 \%$ in limited regions near range changes which has since been fixed in hardware, and a 1-4\% effect due to crosstalk between channels. The crosstalk has been investigated and a correction is now in place. A residual 
non-linear effect of $1-2 \%$ is observed at the gain range changes. The measurement and correction for this are described in the following chapter.

The EMC uses a series of calibrations to ensure accurate operation. At low energies a $6.13 \mathrm{MeV}$ radioactive source (Flourinerts is irradiated with low energy neutrons resulting in the production of $6.13 \mathrm{MeV}$ photons) provides absolute calibration. A half hour run yields a statistical error of $0.35 \%$. This calibration is performed weekly. At higher energies single crystal calibration uses Bhabha samples, where the energy can be calculated as a polar function of the $e^{ \pm}$angle. In a run of 12 hours, a $0.35 \%$ energy resolution can be achieved. Presently this calibration is performed once a month. Other processes are used to calibrate the EMC at lower energies. Up to $0.8 \mathrm{GeV} \pi^{0} \mathrm{~s}$ are used, while in the region $0.8<\mathrm{E}<9 \mathrm{GeV}$ the correction is derived from single photon Monte Carlo simulations.

The EMC is also equipped with a light pulser system which flashes light directly into the crystals. The frequency of the light is tunable, but the light pulser is mainly used as a diagnostic tool. A light pulser calibration is taken daily.

\subsubsection{Performance}

The energy dependent resolution of the EMC is measured using several different processes. At low energies the resolution is measured using the radioactive source which yields $\sigma_{E} / E=5.0 \pm 0.8 \%$ at $6.1 \mathrm{MeV}$. The energy deposited by the photons from radiative Bhabha scattering can be predicted by the polar angles of the electrons. This process gives $\sigma_{E} / E=1.90 \pm 0.07 \%$ at $7.5 \mathrm{GeV}$. At intermediate energies the resolution can be measured using the mass resolution of $\pi^{0}$ and $\eta$ which decay to two photons of approximately equal energies (each below $2 \mathrm{GeV}$ ), or the decays $\chi_{C 1} \rightarrow J / \psi \gamma$ (average energy around $500 \mathrm{MeV}$ ). Fig. 2.18 shows the energy resolution measured using these methods. Fitting equation 2.3 to the graph gives

$$
\frac{\sigma_{E}}{E}=\frac{(2.32 \pm 0.30) \%}{\sqrt[4]{E(\mathrm{GeV})}} \oplus(1.85 \pm 0.12) \%
$$




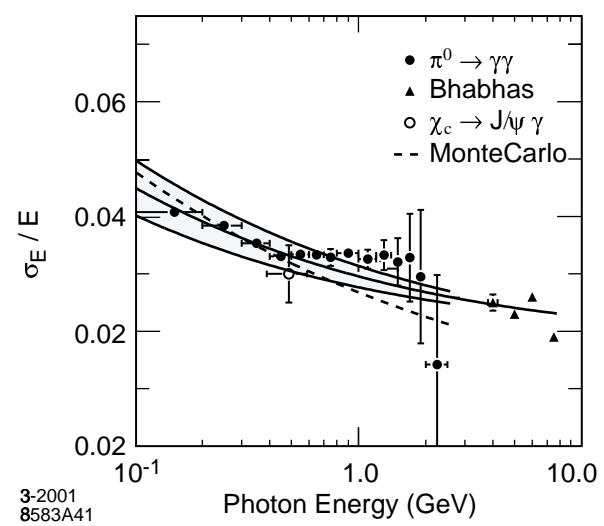

Figure 2.18: The energy resolution of the EMC measured using various processes. The inner solid line represents a fit to equation 2.3 , the two outer lines denote the RMS error of the fit.

These values agree well with Monte Carlo simulations, but are higher than the somewhat optimistic design targets.

Fig. 2.19 shows a plot of the angular resolution that is determined from $\pi^{0}$ and $\eta$ decays to equal energy photons. A fit to the data using an empirical parameterisation gives the following results, which are slightly better than Monte Carlo predictions

$$
\sigma_{\theta}=\sigma_{\phi}=\frac{(3.87 \pm 0.07) \%}{\sqrt{E(\mathrm{GeV})}} \oplus(0.00 \pm 0.04) \mathrm{mrad} .
$$

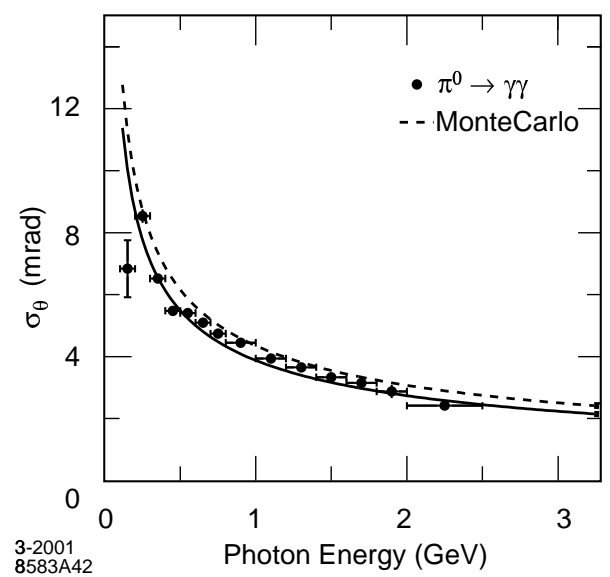

Figure 2.19: The angular resolution of the EMC measured photons from $\pi^{0}$ decays. The solid line is a fit to equation 2.4

The invariant mass of combinations of two photons measured in $B \bar{B}$ events is shown in Fig. 2.20. The mean $\pi^{0}$ mass is measured to be $135.1 \mathrm{MeV} / c^{2}$ and is stable over the full energy range. The $\pi^{0}$ mass resolution is measured to be $6.9 \mathrm{MeV} / c^{2}$. 


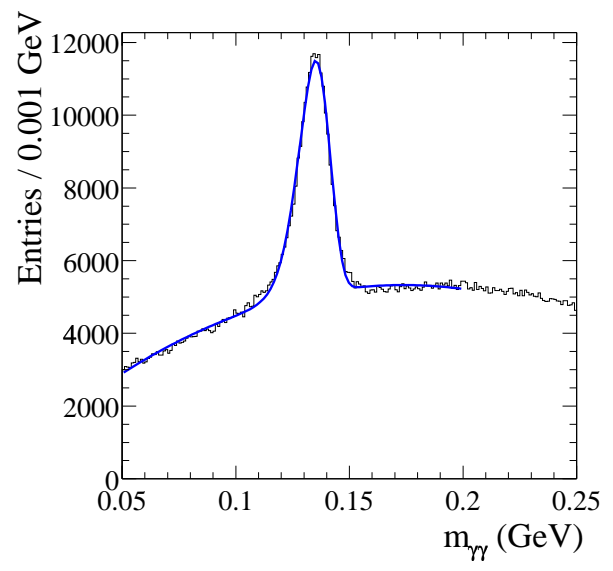

Figure 2.20: The invariant mass of two photon combinations in $B \bar{B}$ decays.

Fig. 2.21 shows the electron ID and pion misidentification achieved using a set of selection criteria [33]. The principal discriminating variable in this measurement is $E / p$ (where $E$ is the energy deposited in the calorimeter). The electron efficiency and pion misidentification are measured using samples of Bhabhas, $e^{+} e^{-} \rightarrow e^{+} e^{-} e^{+} e^{-}$ and $K_{s}^{0}$ decays. A plateau region of $94.3 \%$ efficiency is reached in the momentum range $0.5<p<2 \mathrm{GeV} / c$. The pion misidentification probability is around $0.3 \%$ in the same region.

\subsection{The Instrumented Flux Return (IFR)}

\subsubsection{Requirements}

The purpose of the IFR is to identify muons with high efficiency and high purity, and to detect neutral hadrons. This must be done over a large range of momenta and angles. This helps in the flavour tagging of neutral $B$ meson decays via semileptonic decays, allows the reconstruction of decays such as $J / \psi$ and facilitates the study of semileptonic decays of $B$ mesons, D mesons and $\tau$ leptons.

The IFR is required to provide efficient $\mu$ PID down to an energy of $1 \mathrm{GeV}$. High efficiency and angular resolution are most important for neutral hadrons. The IFR is very large and difficult to access so good reliability and extensive monitoring of the detector performance and electronics are required. 

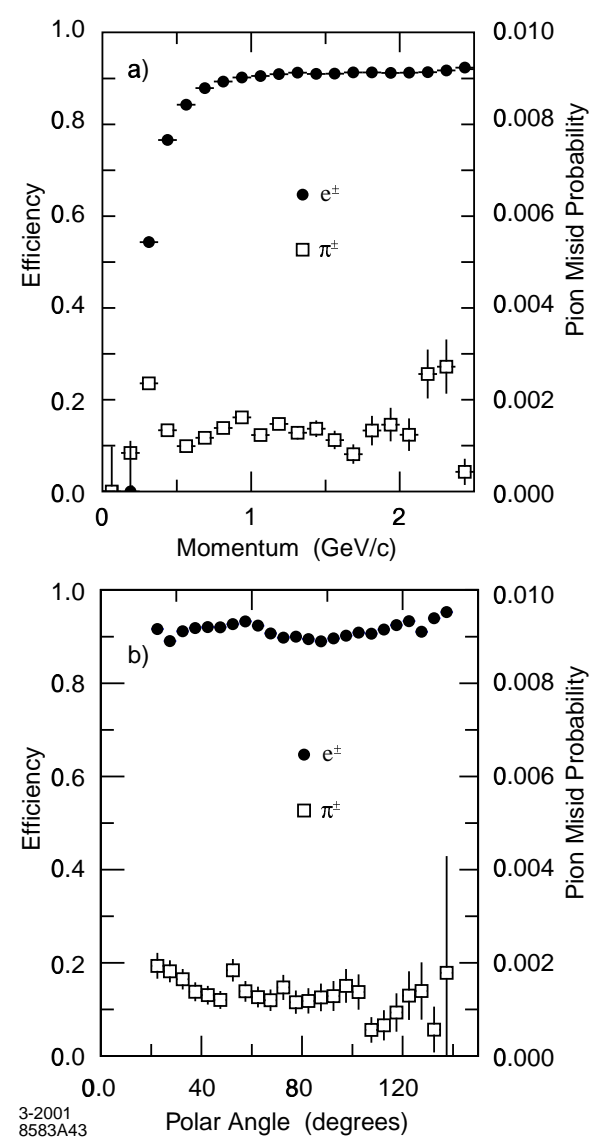

Figure 2.21: The electron identification efficiency (shown on the left scale) and the pion misidentification probability (right scale) as a function of momentum and polar angle.

\subsubsection{Design}

The IFR uses the steel flux return of the magnet as an absorber, see Fig 2.22. The steel is segmented into 18 plates, increasing in thickness from $2 \mathrm{~cm}$ to $10 \mathrm{~cm}$ with distance from the centre of the detector. The gaps in the steel are instrumented with Resistive Plate Chambers (RPCs). There are 19 RPC layers in the barrel section and 18 in the endcaps. There are 2 additional layers of cylindrical RPCs installed between the EMC and the magnet cryostat.

RPCs are relatively low cost devices that offer large signal and fast time response (typically 1-2ns). A cross section of an RPC is shown in Fig. 2.23. They consist of two Bakelite sheets separated by a gap of $2 \mathrm{~mm}$ which is filled with mixture of Argon (56.7\%), Freon (38.8\%) and isobutane (4.5\%) gas. The internal surfaces of the 


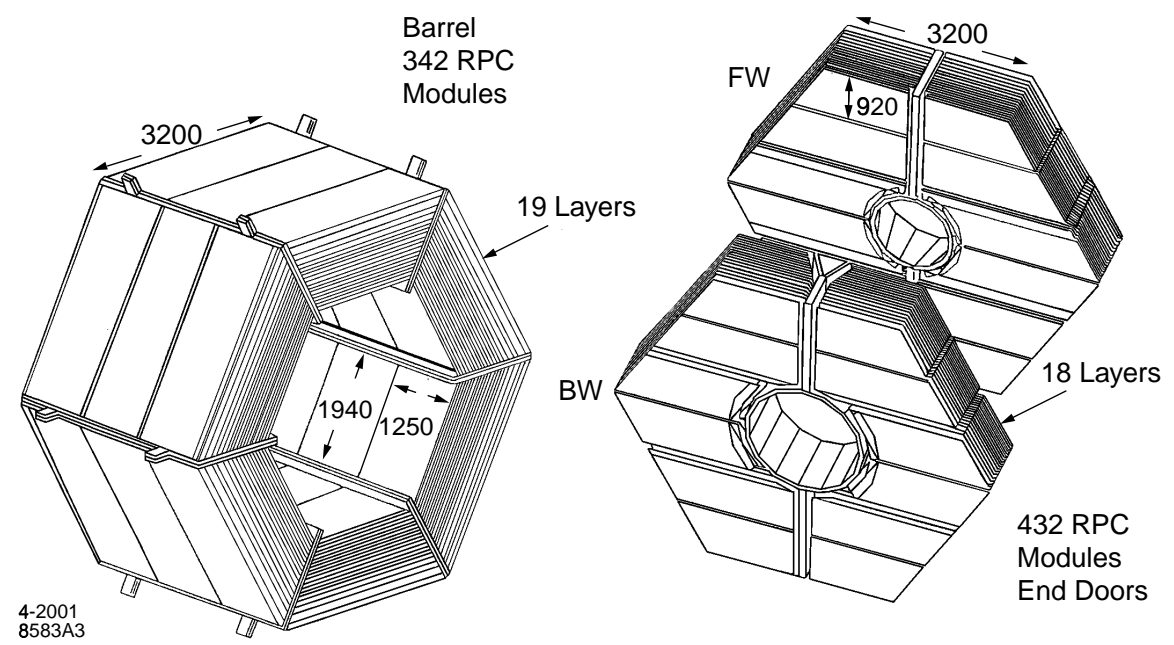

Figure 2.22: Schematic of the IFR.

Bakelite are coated with linseed oil. A high voltage is applied to a graphite coating on the outer surface. The RPCs are operated in limited streamer mode, with the signals being read out capacitively by aluminium strips. Strips on either side of the Bakelite are orthogonal, allowing 2D position measurements. The spacing between the strips in the barrel RPCs is $19.7-32.8 \mathrm{~mm}$ in the $\phi$ direction and $38.5 \mathrm{~mm}$ the $\mathrm{z}$ direction. There are a total of 806 RPCs in the IFR constituting a total active area of $2,000 \mathrm{~m}^{2}$.

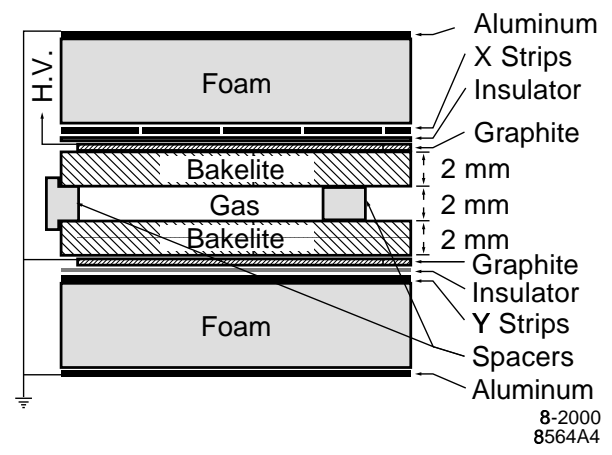

Figure 2.23: Cross section of an RPC module.

The IFR readout electronics provide both position and timing information. Hits from individual strips are grouped together to form clusters, which are themselves grouped to form tracks. The penetration distance of a particle into the IFR is used to infer particle type. 


\subsubsection{Performance}

The efficiency of the IFR is determined for normal collision data and cosmic rays. Individual IFR strips are grouped together to form first $1 \mathrm{D}$ and then $2 \mathrm{D}$ clusters (projecting the clusters through the layers). If a DCH track is observed it is projected through the IFR and nearby clusters are added to the track. An RPC is considered efficient if it records a hit less than $10 \mathrm{~cm}$ from a $2 \mathrm{D}$ straight line fit of the hits. At the start of running $75 \%$ of all active RPCs had an efficiency of above $90 \%$.

The performance of the muon identification is tested using samples of $e^{+} e^{-} \rightarrow e^{+} e^{-}$ $\mu^{+} \mu^{-}, e^{+} e^{-} \rightarrow \mu^{+} \mu^{-} \gamma$. The pion misidentification is measured using pions from three pronged $\tau$ decays and $K_{S}^{0} \rightarrow \pi^{+} \pi^{-}$decays. As shown in Fig. 2.24 an efficiency of close to $90 \%$ can be achieved in the momentum range of 1.5 to $3.0 \mathrm{GeV} / c$ with a fake rate of $6-8 \%$. Using tighter criteria, hadron misidentification can be reduced by a factor of two at the cost of reducing the $\mu$ efficiency to around $80 \%$.

Using multi-hadron decays with a reconstructed $J / \psi\left(\right.$ i.e. $\left.B^{0} \rightarrow J / \psi K_{L}^{0}\right)$ the detection efficiency for $K_{L}^{0}$ is measured to increase roughly linearly with momentum from $20 \%$ at a momentum of $1 \mathrm{GeV} / c$ to $40 \%$ at $4 \mathrm{GeV} / c$. An angular resolution of the order of $60 \mathrm{mrad}$ is obtained. Note that no useful information on the energy of the neutral hadron can be obtained from the IFR.

During the first year of operation a large fraction of RPC modules suffered a significant loss in efficiency which appears to be connected to high temperatures in the IFR. Tests to determine the cause are still continuing but it is believed to be connected to the linseed oil accumulation at various spots. Water cooling has been introduced to the barrel and endcap of the IFR, but future loss of efficiency is still projected to be severe. 24 end door modules have been replaced by new RPCs with a substantially thinner coating of linseed oil. 

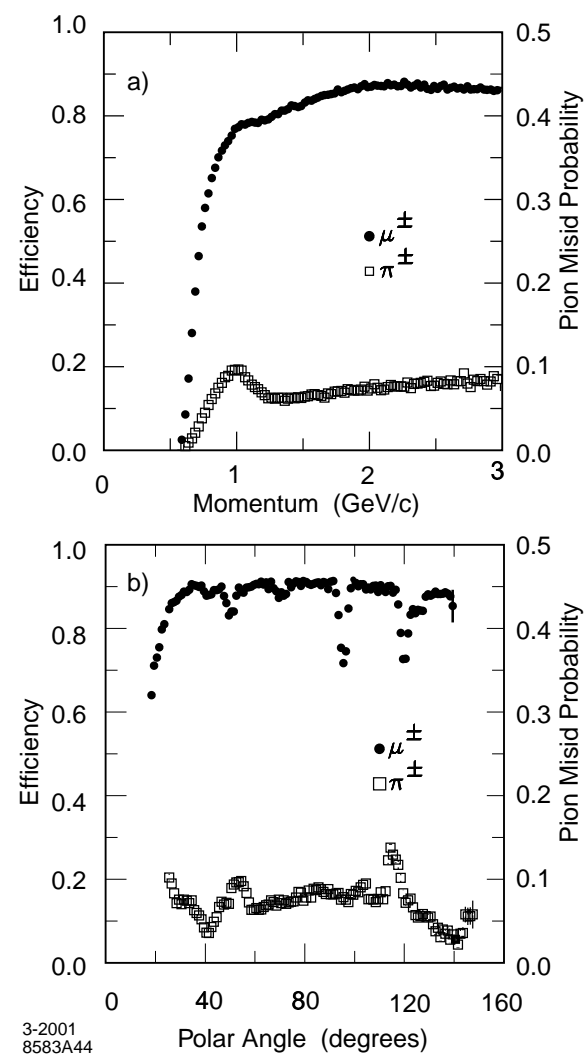

Figure 2.24: Muon identification efficiency (left scale) and pion misidentification probability (right scale) as a function of momentum and polar angle.

\subsection{The Trigger}

\subsubsection{Requirements}

The basic requirement of the trigger system is the selection of events of interest with high, well understood efficiency, while rejecting background events and keeping the total event rate under $120 \mathrm{~Hz}$. Cases with one or more tracks in the drift chamber with $p_{T}>120 \mathrm{MeV} / c$ or at least one cluster with $E>100 \mathrm{MeV}$ have typical rates of $20 \mathrm{kHz}$ from beam-induced backgrounds at design luminosities. The efficiency for $B \bar{B}$ events is required to be over $99 \%$ and at least $95 \%$ for continuum events. The trigger must be robust enough to operate under extreme backgrounds and with dead or noisy channels.

In addition, luminosity and diagnostic studies require accurate prescaled samples of 
some events which may fail the trigger selection. The trigger should contribute no more than $1 \%$ of the total dead time of the detector.

\subsubsection{Design}

The trigger system is divided into two layers. A Level 1 (L1) fast hardware trigger and a Level 3 (L3) trigger which is implemented in software. This system has a built-in redundancy to measure and monitor trigger efficiency and is designed to operate at up to 10 times the design luminosity.

The L1 trigger is configured to have a typical output rate of $1 \mathrm{kHz}$. It uses information from three of the detector subsystems previously described, the $\mathrm{DCH}$, the EMC and the IFR. The DCH trigger (DCT) and the EMC trigger (EMT) are both able to satisfy the trigger requirements independently, so provide redundancy which is valuable for trigger efficiency determination. The IFR trigger is used for triggering on $\mu^{+} \mu^{-}$events and cosmic rays. Data from each of the individual triggers are processed separately and then summary information (trigger primitives) is sent to the global trigger (GLT). This processes the information and, if required, sends a L1 Accept to the Fast Control and Timing System (FCTS). The GTL sets different trigger flags depending on the event information. The DCH and EMC front end electronics take around $2 \mu$ s to send information to the DCT and EMT. DCT and EMT processing takes 4-5 $\mu \mathrm{s}$, followed by $3 \mu \mathrm{s}$ of GTL processing and then a further $1 \mu \mathrm{s}$ for the signal to propagate to the FCTS. These steps all need to be accomplished within the $12.8 \mu$ s buffer capacity.

The primitives output by the DCH are candidate tracks. First track segments (in arrays of 8 cells) are found and then combined into tracks. The efficiency for finding a track segment is $97 \%$ and a position resolution of $\sim 900 \mu \mathrm{m}$ is achieved. The EMT produces primitives by summing energy deposited in 280 EMT towers (in the barrel each tower consists of 24 crystals in an $8 \times 3$ array). The EMT efficiency is determined by comparing the triggers to specific energy clusters reconstructed 
offline. The efficiency varies from $10 \%$ to $90 \%$ in the range of 100 to $145 \mathrm{MeV}$ and it reaches $99 \%$ at $180 \mathrm{MeV}$.

The L3 trigger software includes event reconstruction and classification. It reduces the $\mathrm{L} 1$ output to a total rate of around $120 \mathrm{~Hz} ; 90 \mathrm{~Hz}$ for physics events and $30 \mathrm{~Hz}$ of other special events categories. The L3 trigger has access to the full data as well as the L1 triggers. It improves upon the reconstruction methods used in the L1. It then uses the improved cluster and tracks to filter the events into various categories and reduce background. Examples include removal of background tracks by reconstructing the minimum distance between the track and the IP, and Bhabha rejection by vetoing one and two prong events with stringent $\mathrm{EMC} E / p$ criteria.

\subsubsection{Performance}

The L1 hardware has been very stable. A typical L1 rate of $1 \mathrm{kHz}$ is composed of $\sim 130 \mathrm{~Hz}$ Bhabhas and annihilation physics events, $100 \mathrm{~Hz}$ of cosmic rays and $20 \mathrm{~Hz}$ of random beam crossing triggers. The rest of the rate is composed of lost particles interacting with the beam pipe and other components. Fig. 2.25 shows the minimum distance between the track and the IP reconstructed in the L3 for L1 tracks. The peaks at $\pm 20 \mathrm{~cm}$ corresponds to a flange of the beam pipe.

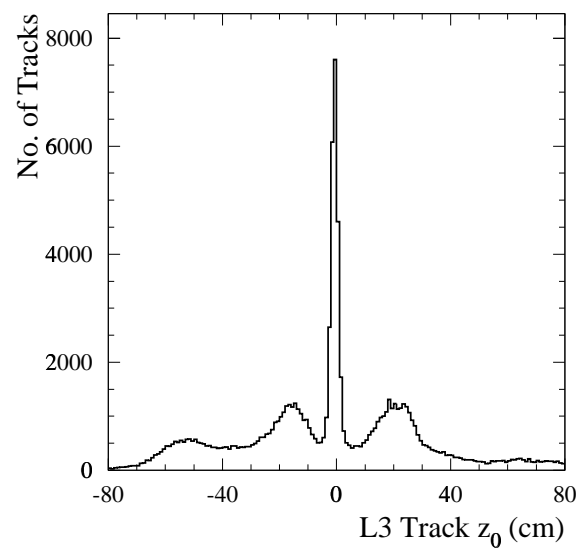

Figure 2.25: The distance of closest approach of single L1 tracks to the IP in the $\mathrm{z}$ direction as reconstructed by L3. 


\begin{tabular}{c|c}
\hline \hline Event type & Efficiency (\%) \\
\hline$B \bar{B}$ & $>99.1$ \\
$B \rightarrow \pi^{0} \pi^{0}$ & 99.1 \\
$B \rightarrow \tau \nu$ & 97.8 \\
$c \bar{c}$ & 98.9 \\
$u \bar{u}, d \bar{d}, s \bar{s}$ & 95.8 \\
$\tau \tau$ & 92.0 \\
\hline \hline
\end{tabular}

Table 2.2: L1 and L3 combined efficiency for various physics processes, derived from Monte Carlo simulations.

For a typical run the L3 event composition is made up of $13 \%$ of desired physics events and $40 \%$ calibration and diagnostic samples. The average L3 processing time is $8.5 \mu$ s per event per computer CPU. A L1 output rate of $2.7 \mathrm{kHz}$ saturates the L3 processors, well above the $2 \mathrm{kHz}$ design value.

Table 2.2 shows the combined efficiency of the L1 and L3 triggers for various event types. The design goal of an efficiency greater than $99 \%$ for $B \bar{B}$ events is met. 


\section{Chapter 3}

\section{EMC Calibrations}

\subsection{Introduction}

This chapter details the measurement and correction of non-linearities in the response of the EMC readout electronics. Specifically it examines the non-linearities that occur at changes between amplifier gain ranges. The chapter will begin with a discussion of the EMC electronics and its calibration. There will be a brief review of the non-linearities discovered in the EMC electronics since the beginning of operations and the previous fixes applied to remedy them. Focus will then be given to the outstanding non-linearities associated with the switches in amplifier gain ranges. The characteristics of the effect will be detailed, along with the calibration used to correct for the effect.

\subsection{The EMC Electronics}

A schematic of the EMC readout electronics is shown in Fig. 3.1 and a detailed description of the front end electronics can be found in [35]. The crystals are read out by two silicon photo-diodes $(\mathrm{A}$ and $\mathrm{B})$ and amplified into a $\times 1$ and $\times 32$ gain analogue signal by preamplifiers located on a small printed circuit board on the back 
of each crystal. After some pulse shaping the analogue signal is then transported via shielded ribbon cables to mini-crates located on the end flanges of the detector. The backward 24 theta rings of the barrel are read out on the rear face of the detector, the forward 24 barrel theta rings and the 8 endcap theta rings are read out at the front face. Crystals in the last 27 theta rings of the barrel (all of the backward barrel and 3 theta rings of the forward barrel) are read out by higher gain preamplifiers (a factor of 1.5 greater) than those in the rest of the detector. This is to compensate for the asymmetric collision in the BABAR detector.

The analogue signal from sets of 12 crystals are input into Analogue to Digital Boards (ADB) which contain Custom Analogue Range Encoder (CARE) and Analogue to Digital Converter (ADC) circuitry. An ADB contains 3 CARE chips, each responsible for 4 channels. The CARE chip takes the analogue signal from the preamplifiers on the crystal and further amplifies it into $\times 256(8 \times 32), \times 32(1 \times 32), \times 4(4 \times 1)$ and $\times 1(1 \times 1)$ stages (corresponding to the energy ranges $0-50 \mathrm{MeV}, 50-400 \mathrm{MeV}$, $0.4-3.2 \mathrm{GeV}$ and $3.2-13.0 \mathrm{GeV}$ respectively). Using a latched comparator circuit the CARE chooses the appropriate amplification range by comparing the four gain outputs to reference voltages set at $90 \%$ of the full scale for each range. The highest gain output not exceeding the appropriate reference voltage is chosen. The selected output is then digitised using a 10 -bit, $3.7 \mathrm{MHz}$ ADC. Using this system a 10 bit ADC along with two digital range bits (indicting which gain range was selected by the CARE) provide the required effective dynamic range of 18 bits. The CARE chip is configurable to use either diode (A or B), or the average of both as an input; the default setting is the average of the two. It is possible to configure the CARE chip to use individual gain ranges for calibration and diagnostic purposes.

In the barrel section a set of 6 ADBs constitute a mini-crate and are connected to an Input Output Board (IOB). For the endcaps there are 4 ADBs per mini-crate. On the IOBs the 12 bit samples from 24 channels (2 ADBs) are multiplexed into a 1.5 Gbit/s optical fibre link and transported to a ReadOut Module (ROM) located in the electronics hut, off the main detector. In the ROM the 12 bit samples are decoded and fed into a LookUp Table (LUT) which outputs a 16 bit absolute energy 
reading. The data are stored in a pipeline and copied to the Level 1 trigger. An EMC DataAcquisition (DAQ) crate corresponds to 10 mini-crates. There are a total of 10 crates in the EMC ( 8 barrel and 2 endcap).

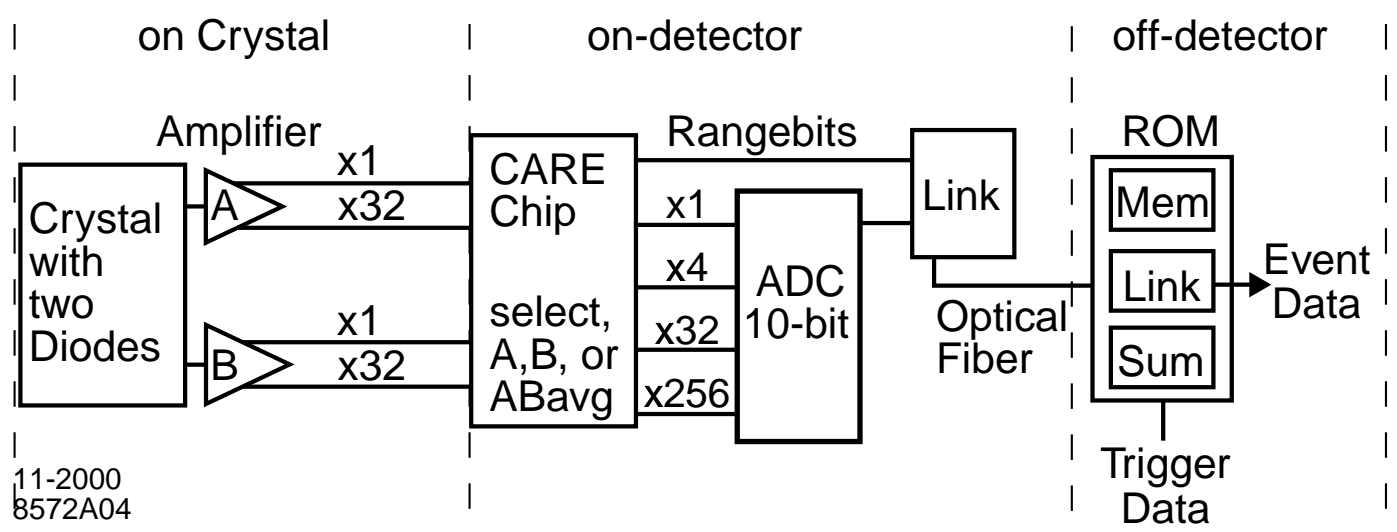

Figure 3.1: The EMC readout electronics chain.

\subsection{Electronics Calibration}

The electronics calibration is used to determine the LUT used to convert the raw $\mathrm{ADC}$ and range information into an absolute energy measure in the ROMs. Under normal operation, charge is injected into the preamplifiers by the photo-diodes as they absorb scintillation light from the crystals. In an electronics calibration charge is injected directly into the preamplifiers input using calibration circuitry built into the front end electronics. Measuring the ADC output for the well defined input charge allows the calibration of the whole electronics chain.

Charged pulses are produced by discharging one of two capacitors located on the preamplifier board. These pulses are shaped to resemble pulses of light from CsI crystals seen by the photo-diode. Which capacitor is used, the large $1.8 \mathrm{pF}$ or the small $220 \mathrm{fF}$, is determined by a control command from the CARE chip. The voltage on the capacitors can be varied from $0-2 \mathrm{~V}$ using a precision 16 bit Digital to Analogue Converter (DAC) located on the IOB. Using the two capacitors the full energy range of the system can be calibrated. Unlike in normal data taking, during the electronics calibration the gain range output by the CARE is selected systematically. 
A set of $100 \mathrm{ADC}$ vs DAC measurements are taken for each of the CARE gain ranges. The $\times 1$ and $\times 4$ ranges are measured using only the large capacitor and the $\times 256$ using only the small capacitor. Measurements using both capacitors separately are taken for the $\times 32$ range, allowing the ratio of the capacitance to be determined. If the response of the EMC electronics were linear the value of the ADC measured in a particular gain range $R$ using the small capacitor would be given by

$$
A D C_{R}=D A C \times g_{R}+p_{R}+O_{s} \times g_{R}
$$

and using the large capacitor by

$$
A D C_{R}=D A C \times r g_{R}+p_{R}+r O_{l} \times g_{R}
$$

where $g$ is the gain of the preamp, $p$ is the pedestal value, $\mathrm{r}$ is the ratio of the two capacitors and $O_{s}\left(O_{l}\right)$ is the initial DAC offset on the small (large) capacitor. The pedestal values are determined in a separate calibration by taking random triggers with no beams in the detector. Standard non-linear fitting techniques are used to determine the remaining parameters from a global fit of the data.

In order to account for non-linearities in the electronics response (which turn out to

be significant in the $\times 1$ and $\times 4$ gain ranges) a table of residuals are determined using the fit results and the measured $\mathrm{ADC}$ vs DAC data. The measured fit parameters and residual tables are then used to create a LUT which maps the 10 bit ADC output for each range onto the charge input into the photo-diodes.

Note, in practise the gain of the $\times 1$ and the $\times 4$ ranges are not determined from the ADC vs DAC fit. Instead they are assigned a value by scaling the gain of the $\times 32$ range and correcting using the residual tables. This is a result of the large non-linearities which were observed during the first period of BABAR running.

\subsection{Non-Linearities in the EMC Electronics Re- sponse.}

Since the start of operations three independent sources of non-linearities have been found in the EMC electronics response. These are evident in the energy spectrum of 
data taken during the first year of running as shown in Fig 3.2 and described below. Two of these have since been fixed and a calibration to remedy the third has been developed by the author.

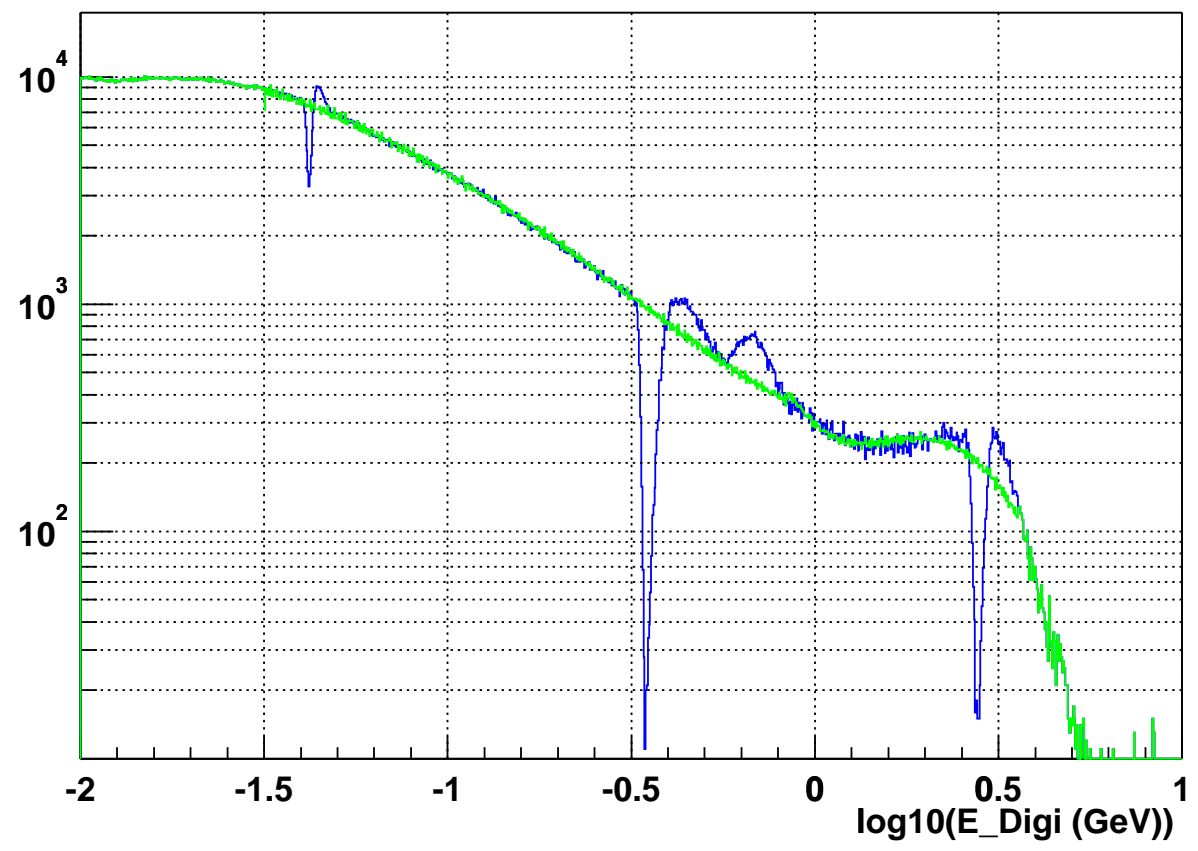

Figure 3.2: The energy spectrum of data of all channels (blue curve) for the first year of running (Run 1 ). The $\times 256-\times 32$ range switch occurs at $\approx-1.4$ which corresponds to an energy of $50 \mathrm{MeV}$. The $\times 32-\times 4$ switch is at $-0.45(400 \mathrm{MeV})$ and the $\times 4-\times 1$ at $0.4(3.2 \mathrm{GeV})$. The overlayed green curve shows the resultant energy spectrum after an offline correction has been applied [1].

\subsubsection{ADB Oscillation}

The largest source of non-linearity was found to be due to an oscillation in the $\mathrm{ADB}$, or "ringing". The ADB was driven into an oscillating state when the board was running under high load, for example during an electronics calibration when all channels fire simultaneously. This led to energy non-linearities of up to $10 \%$ in the observed response. This effect was found to be responsible for most of the non-linearity that occurs at the changes between CARE gain ranges (which occur at $50 \mathrm{MeV}, 400 \mathrm{MeV}$, and $3.2 \mathrm{GeV}$ ) and partially responsible for the oscillatory behaviour after the $\times 32-\times 4$ switch $(400-800 \mathrm{MeV})$. This problem was fixed by 
adding extra capacitance to the ADB during the 2000/2001 winter shut down, the Run1/Run2 boundary.

\subsubsection{Crosstalk}

The second largest contribution was found to be due to crosstalk between the electronics channels. This effect only sets in when the $\times 32$ range saturates, corresponding to a energy of around $400 \mathrm{MeV}$. Combined with the ADB ringing this effect is responsible for oscillatory behaviour in the digi spectra after the switch between the $\times 4$ and $\times 32$ ranges, between $400 \mathrm{MeV}$ and $800 \mathrm{MeV}$. The non-linearities are of the order of $4 \%$.

During an electronics calibration all channels are pulsed simultaneously, resulting in maximum crosstalk between channels. As a result the LUT derived from the electronics calibrations has a non-linear response which leads to a non-linearity in the observed data spectrum. In normal data taking it is rare for more than one or two crystals to exceed the relevant energy scale $(\approx 400 \mathrm{MeV})$ required to induce crosstalk. As a result the crosstalk effect is 6 to 12 times smaller in normal data taking than during an electronics calibration.

The crosstalk effect was investigated during the winter 2000/2001 shutdown [36]. Hardware was developed to allow the crosstalk induced in channels neighbouring a

pulsed crystal to be measured. A correction was developed from these measurements which has been applied as a standard component of the electronics calibration since the beginning of Run2.

The energy spectrum of data taken during Run2 is shown in Fig. 3.3. The main non-linearities evident in the Run1 data appear to have been successfully removed, but non-linearities of around $2 \%$ can still be observed at energies corresponding to switches in CARE ranges. These non-linearities and their correction are the subject of the remainder of this chapter. 


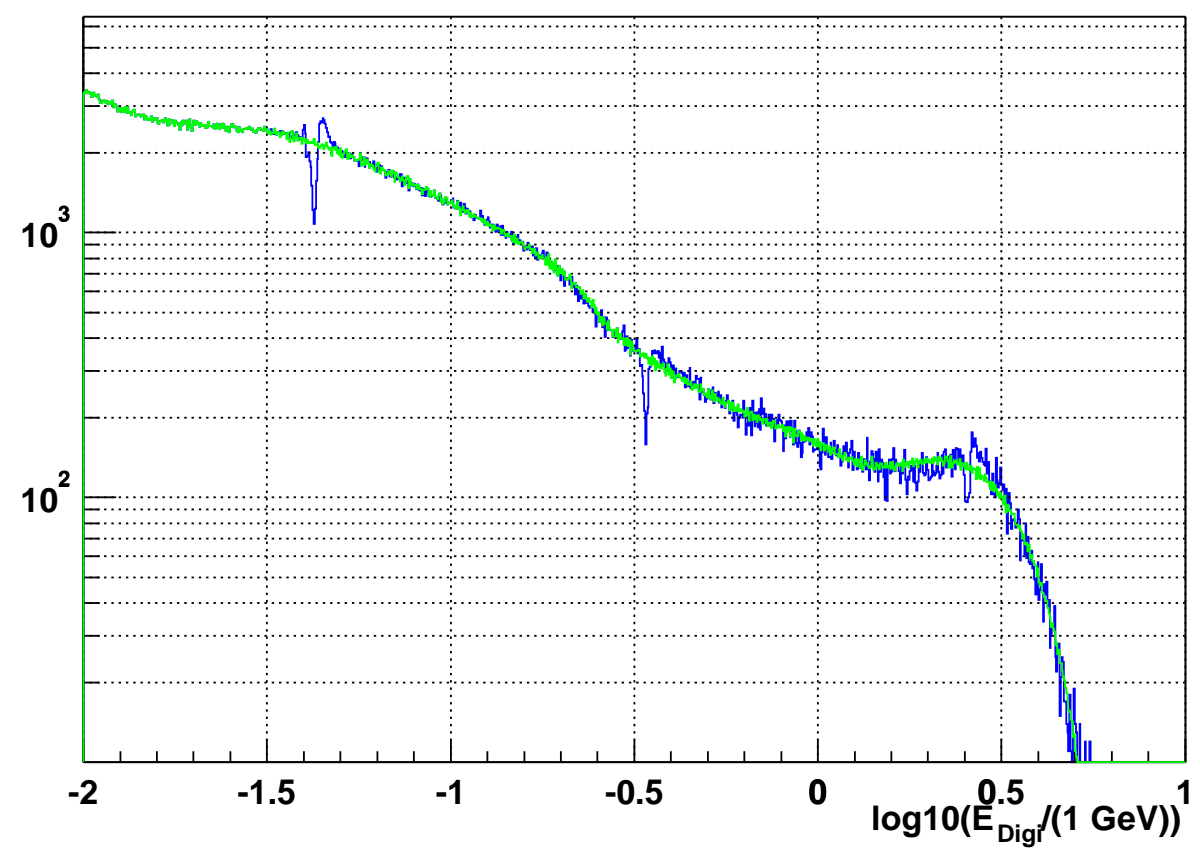

Figure 3.3: The energy spectrum (blue curve) of all channels for Run2 (2002) data. The overlayed green curve shows the resultant energy spectrum after an offline correction has been applied [1].

Note, since the corrections for the ADB ringing and the crosstalk were both deployed at the same time, the extent to which either individual problem contributed to the observed non-linearities is not obvious, especially in the region of the $\times 4-\times 32$ range change. In order to correct data taken during Run1, before the previously discussed corrections were applied, an offline correction was developed [1].

\subsection{Remaining Non-Linearities at Range Switches}

After the the application of the fixes mentioned in the previous section there is still a residual non-linearity evident in the energy spectrum at energies which correspond to the changes in CARE gain range. This section looks at the characteristics of these non-linearities and describes the method developed to measure them.

As previously mentioned, during normal data taking operation the CARE chip selects which of the four gain ranges to output by comparing the signal to reference 
voltages (auto-ranging mode), whilst during an electronics calibration the CARE is set to use only one gain range at a time (fixed mode operation). A LUT is derived from the electronics calibration by fitting the ADC vs DAC measurements taken in each fixed gain range. As the LUT is determined using measurements from the electronics calibration, i.e. the fixed mode of operation only, any effects that occur in the auto-ranging mode that are not replicated in the fixed mode will not be compensated for in the LUT and lead to non-linearities in the observed energy spectrum.

A second source of non-linearities at range changes would occur if any of the parameters determined by the electronics calibration for each separate gain range were inaccurate. This would lead to the two LUTs outputing different values for the same input energy.

To investigate the non-linearity a modified electronics calibration was developed. There are in essence three separate stages

- In order to investigate if there is any effect at the range switch it is first necessary to determine the point at which the range change occurs, i.e. the 2 bit range information from the CARE. In order get this information a modified LUT was constructed which simply flagged the gain range information. The DAC value at which the range switch occurs can then be found.

- Once the DAC value of the range change is found the surrounding DAC values are scanned using the auto-ranging mode of operation. The LUT used is that determined by the full electronics calibration, i.e. the same as used during data taking.

- The same DAC values are then scanned using the same LUT, but with the CARE fixed to the lower gain range. These measurements are then compared to those taken using the auto-range mode. Any non-linearities should be evident in the difference of the two measurements. 
Fig. 3.4 shows the LUT counts vs DAC count across the $\times 256-\times 32$ range change for a single channel using both the auto-range mode and the CARE fixed to $\times 32$ gain range. The range change occurs at $1110 \mathrm{DAC}$ counts. Before this point the autorange mode selects the output from the $\times 256$ range and uses the LUT determined for this range. After the range change the CARE selects output from the $\times 32$ range and uses the appropriate LUT. Any discrepancy between the auto-range and fixed output before 1110 DAC counts is an indication that the LUTs for each for the two ranges give differing values for the DAC input, i.e. the parameters derived by the electronics calibration do not adequately describe the response of at least one of the ranges in this area. The figure shows a clear discrepancy after the range change. This is an indication of a physical difference for the signal output between the autoranging and fixed mode output. The discrepancy peaks at the range change and falls off as it moves into the $\times 32$ range.

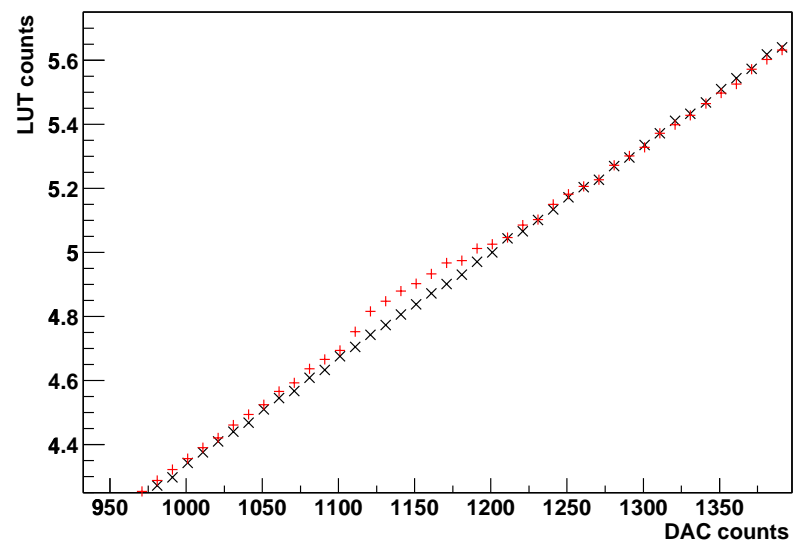

Figure 3.4: The plot shows the LUT count against DAC response for the same channel using two different modes of data taking. The red $(+)$ points show data taken in auto-ranging mode and the black $(x)$ using the $\times 32$ amplifier. A deviation between the two sets of data is apparent at the point where the auto-ranging mode switches between the $\times 256$ amplifier and the $\times 32$ amplifier at around 1110 DAC counts.

Measurements were taken for all channels across different crates in the detector. Fig. 3.5 shows the percentage deviation between the response in the fixed mode and the auto-ranging mode, i.e. $100 \times\left(\mathrm{LUT}_{\text {fixed }}-\mathrm{LUT}_{\text {auto }}\right) / \mathrm{LUT}_{\text {fixed }}$, against $\mathrm{DAC}$ values for all channels in crate 3 (forward barrel), crate 7 (backward barrel) and crate 8 (endcap). The DAC values for each channel are adjusted so that $\mathrm{DAC}=0$ corresponds to the point of the range change. 

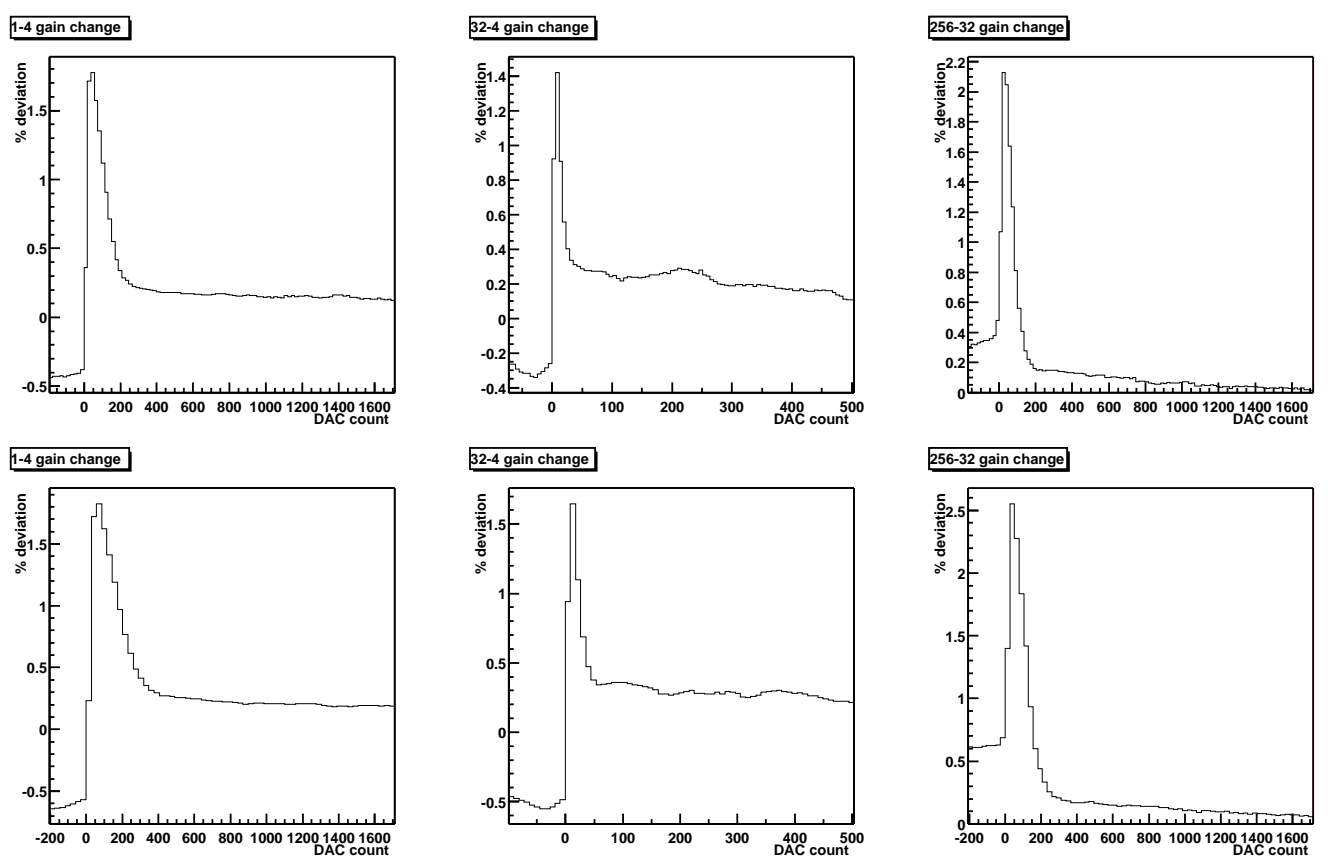

\section{$32-4$ gain change}

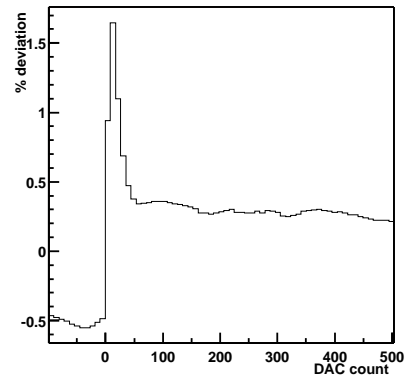

$32-4$ gain change

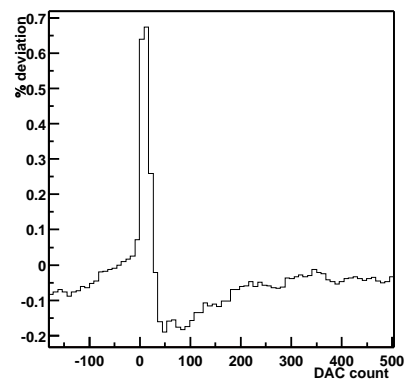

256-32 gain change

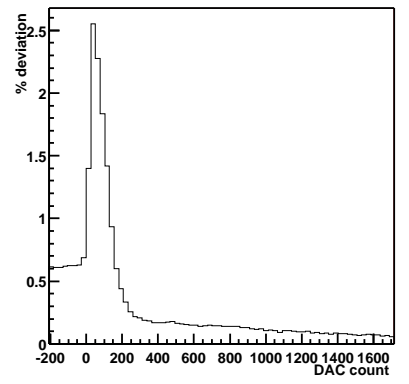

256-32 gain change

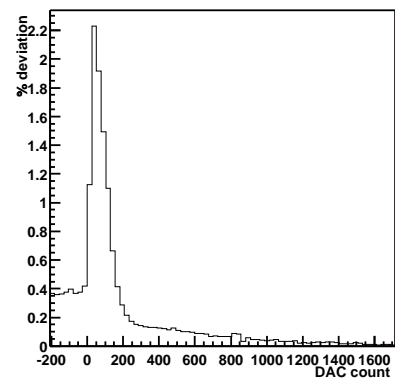

Figure 3.5: The plots show the average percentage deviation between the auto-ranging mode of operation and the fixed amplifier mode across the amplifier switch for all three gain ranges and for all channels in one crate. The topmost set of plots correspond to crate 7 , which is situated in the forward barrel and has high gain preamplifiers. The middle set of plots show crate 3 data, a forward barrel crate with low gain preamplifiers. The lower plots correspond to crate 8 in the end cap.

From the figure we see that,

- the average size of the effect after the range change is of the order of $2 \%$, which corresponds to approximately $4 \mathrm{ADC}$ counts, and is localised to the area immediately following the range change.

- The effect is present in all crates. It occurs at all three range changes, though the $\times 32-\times 4$ switch shows slightly different behaviour to the other two. 
- The deviation from zero before the range switch $(\mathrm{DAC}<0)$ shows a misalignment between the LUTs for the two different amplifier ranges. This will also contribute to any gaps observed in the digi spectra. There are indications that this effect is at least in part due to the crosstalk correction procedure (discussed later).

\subsubsection{Structure in Crates}

A mini-crate is made up of six ADBs, each containing 12 channels. Each successive $\mathrm{ADB}$ corresponds to channels that are further inside the detector (for crates located in the barrel). Fig. 3.6 shows the maximum deviation for each channel in a minicrate averaged over all mini crates in crate 7 for the $\times 1-\times 4$ transition; the higher the channel number, the further into the detector is the channel. Also shown is the deviation averaged over individual ABDs in the mini-crate. The plots seem to suggest that the non-linearity increases with depth in the barrel. In general the effect is the same size in individual ADBs.
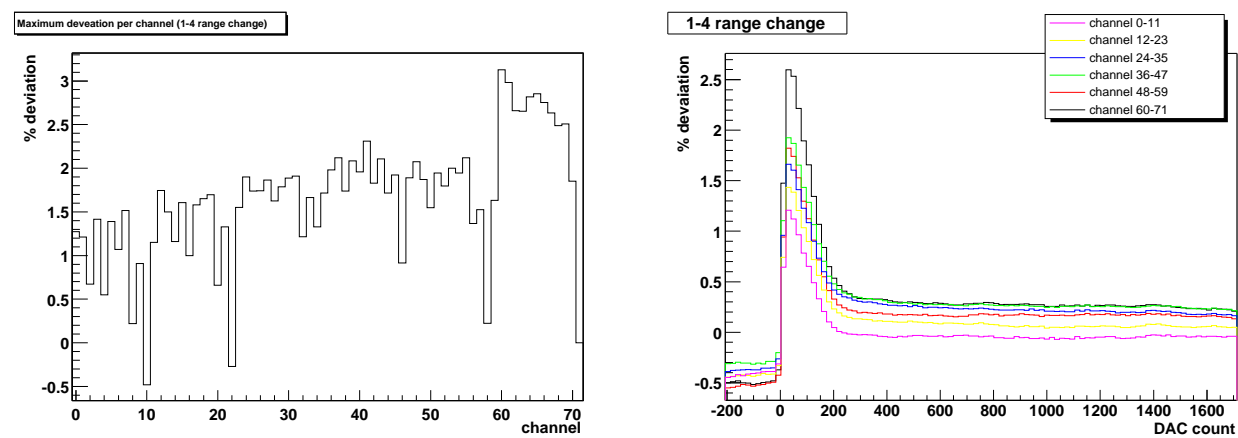

Figure 3.6: The left plot shows the maximum deviation per channel averaged over mini crates in crate 7 for the $\times 1-\times 4$ range switch. The right plot shows the average deviation separated into ADBs (12 channels). Higher channels are further into the barrel than lower channels.

\subsubsection{Crosstalk Corrections}

Documentation on the crosstalk correction can be found elsewhere [36]. The basic principle is that the crosstalk observed when only half the channels are pulsed is 
scaled to that measured for single pulsed channels and then subtracted from the normal electronics calibration. The actual calibration is done offline after the original electronics calibration. Fig. 3.7 shows the deviation per channel averaged over both even channels and odd channels in crate 7 , both with and without the crosstalk correction. From this figure it can be seen that for the even channels the $\times 1$ and the $\times 4$ LUT align well, but not for the odd channels. Before the crosstalk correction the LUTs align for all channels.
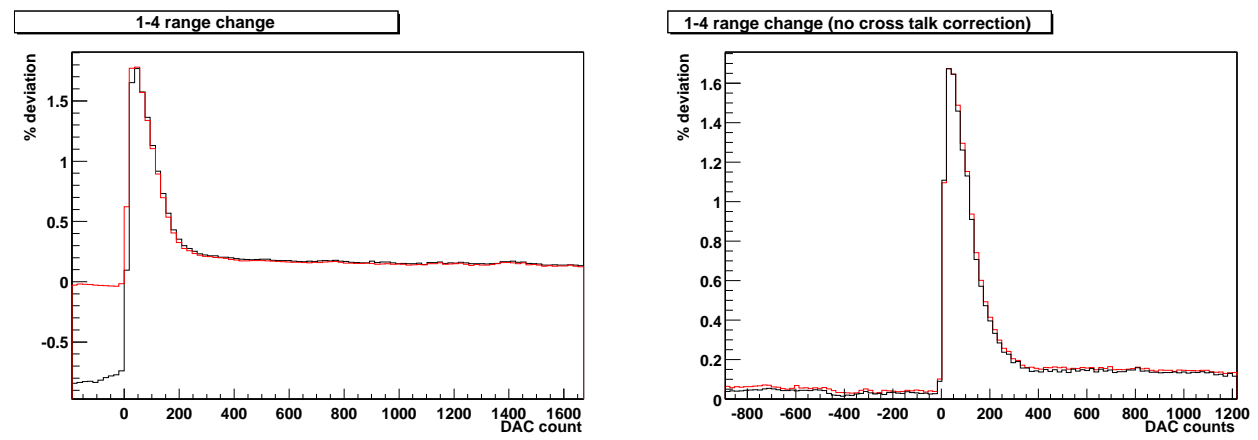

Figure 3.7: Each plot shows the average deviation between fixed and auto ranging operation for odd (black) and even (red) channels. For the plot in the left the crosstalk corection has been applied. There was no cross talk correction for the plot on the right

A new method of correcting for crosstalk has recently been developed and it remains to be seen if this artifact still persists in the new method.

\subsection{Non-Linearities Correction}

Having observed a non-linearity between the auto-ranging and fixed mode of operation at the range changes the next step is to engineer a correction for it. The way this is achieved is by measuring the size of the effect in each channel and accounting for it in the LUTs.

A calibration was designed to measure the discrepancy between the auto-ranging and fixed range modes of operation at each of the three ranges. The method is similar to that previously outlined. The electronics is first configured to use the fixed gain mode and measurement of LUT counts are taken for 100 DAC values 
around the range change. The LUT tables used for this are the standard tables used for data taking, with crosstalk corrections already applied. The same DAC values are then used to measure the response in the auto-ranging mode. Residual tables are then constructed and stored for each of the three range changes for every channel. These tables are used to adjust the LUT constructed for the lower gain range at each range switch (i.e. for the $\times 256-\times 32$ switch the LUT for the $\times 32$ range is adjusted).

Fig. 3.8 shows the deviation between the auto-ranging and fixed range response both before and after the calibration described above. The calibration successfully removes the main peak seen immediately after the range change. This correction procedure makes no attempt to account for any difference between the two LUT before the range change.

This correction procedure has been incorporated into the standard electronics calibration since April 2002.
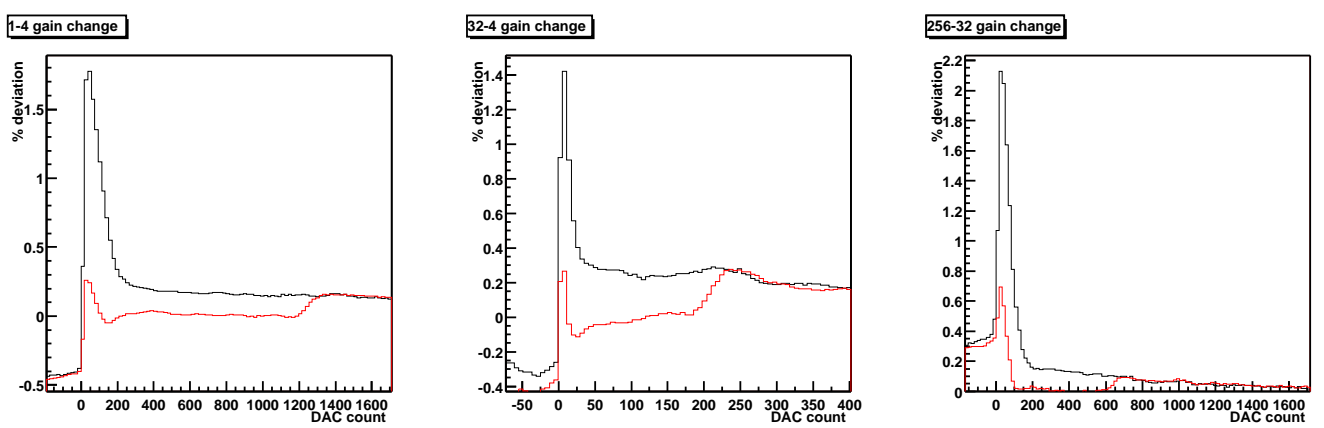

Figure 3.8: The plots show the average percentage deviation for each of the 3 gain range switches for crate 7 before (black) and after the correction (red).

\subsection{Corrected Digi Spectra}

Examples of single channel energy spectra are shown in Fig. 3.9, both before and after the new calibration was implemented in the standard electronics calibration. The gaps at the range changes are clearly visible in the energy spectra before the correction, and effectively removed after. 
The energy spectra for Bhabha events averaged over theta rings both before and after the auto-range correction are shown in Fig. 3.10. The effectiveness of the correction appears to depend on the position of the crystal in the detector. The correction appears to work better toward the outside of the barrel.

\subsection{Conclusions}

Non-linearities are observed in the regions where the output of the CARE chip switches from one amplifier to another. This is due at least in part to a discrepancy that occurs between data taken in auto-ranging compared to fixed range mode. The size of the resultant non-linearity is around two percent and is localised to the region immediately following the range switch. The cause of this effect remains unknown, but a calibration has been developed to compensate for it. 

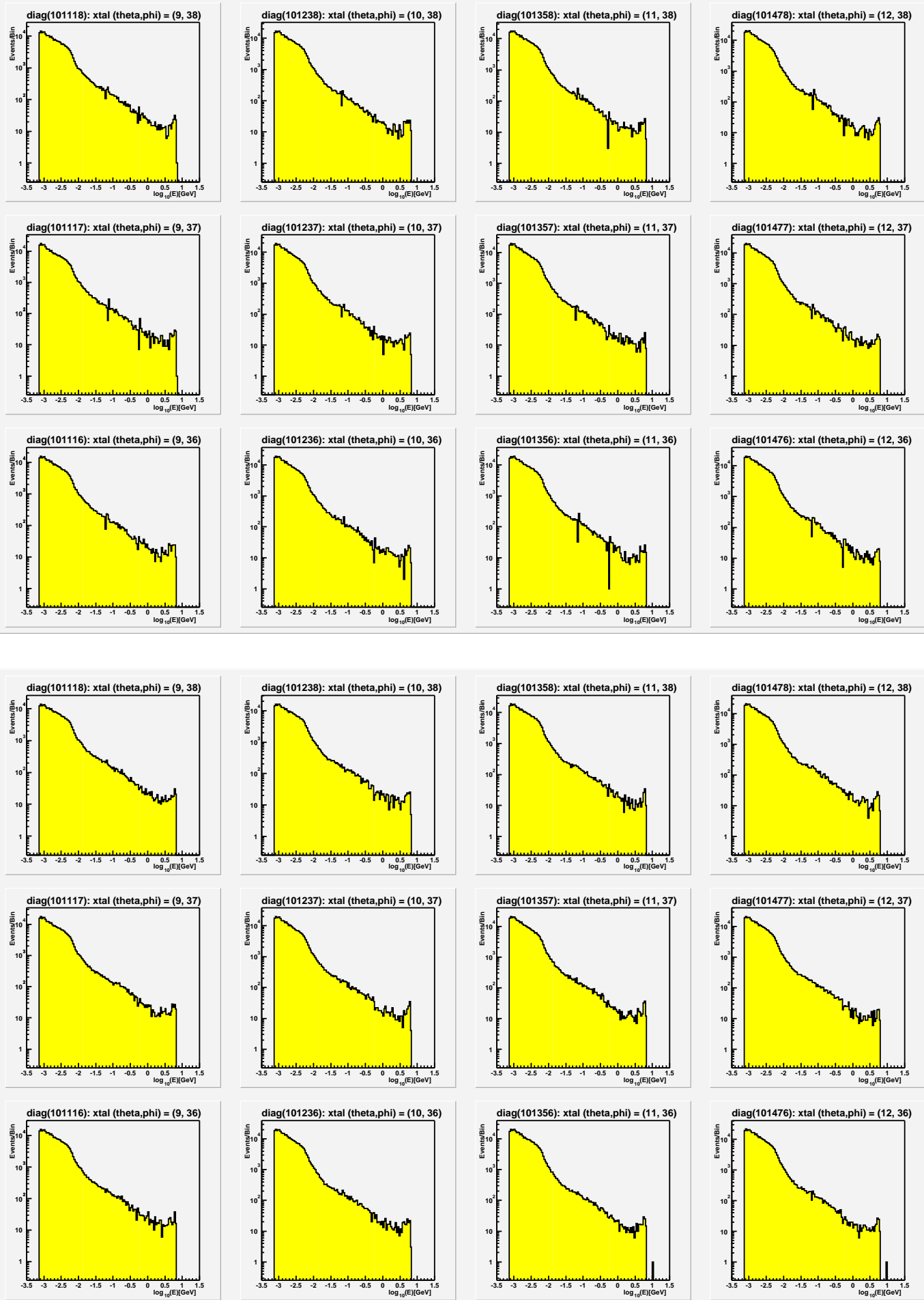

Figure 3.9: The plots show single crystal energy spectra for data taken during Run2. The upper set of 12 plots (which corresponds to 1 ADB) show the energy spectra before the range change correction is applied. The lower plots show the same channels after the correction. 

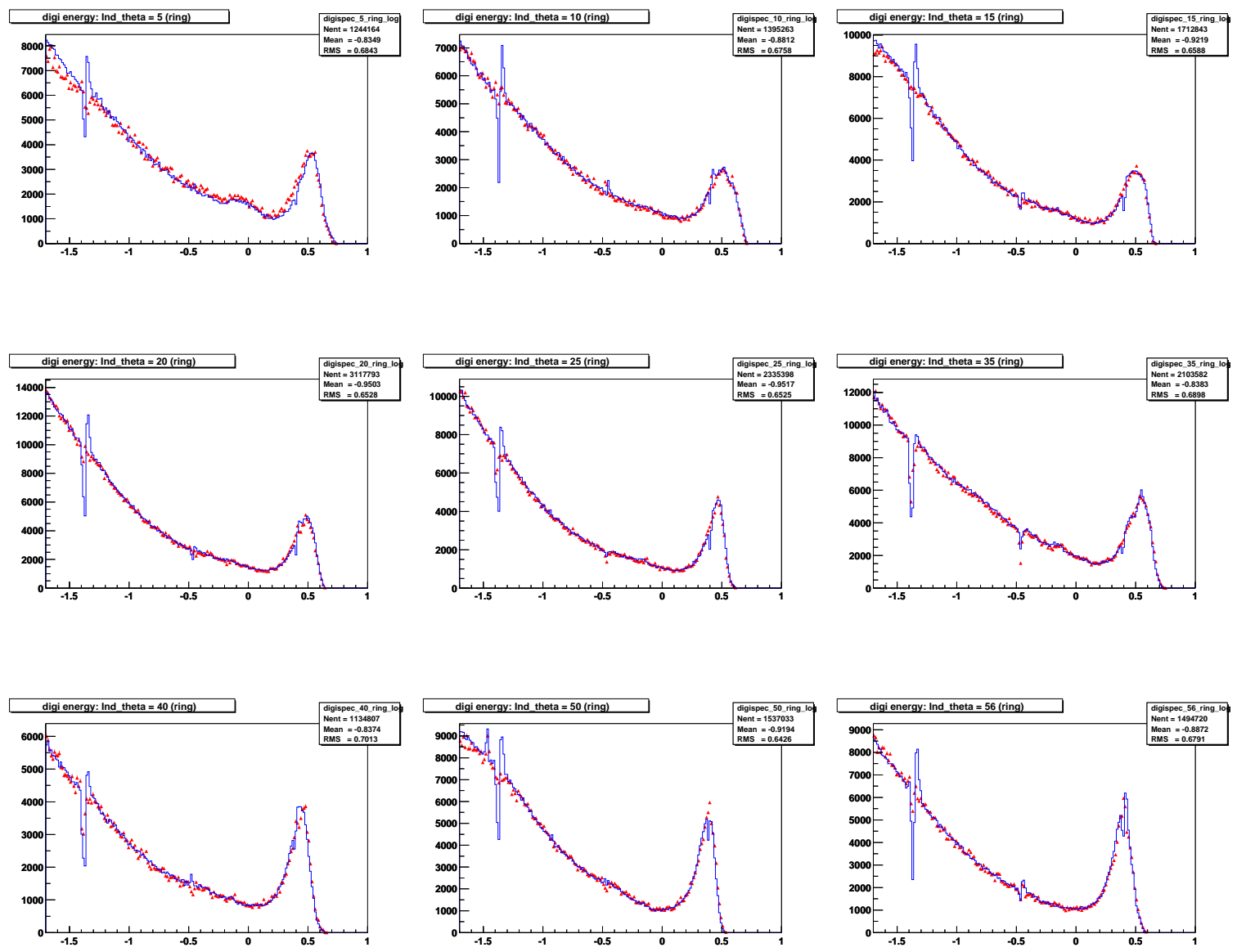

Figure 3.10: The plots show the energy spectra taken from Bhabha events separated into theta rings. The $\mathrm{x}$-axis units are $\log _{10}[\mathrm{GeV}]$. The blue plots were taken before the auto-range correction was implemented and the red plots were after the implementation. 


\section{Chapter 4}

\section{Event Selection and Neutrino Reconstruction}

\subsection{Introduction}

This chapter will detail the event selection and reconstruction used in order to obtain a sample of $B \rightarrow X_{u} l \nu$ decays. The fundamental track and neutral selection adopted is discussed along with candidate reconstruction. The main advantage of exclusive reconstruction over inclusive techniques is the higher background suppression that can be achieved. The variables used to gain this increase, such as the constraint on the missing mass of the neutrino, will be introduced in this chapter. The analysis is very sensitive to the quality of the data/Monte Carlo simulation agreement as the eventual shapes used to fit the sample will be taken from Monte Carlo simulation. In order to test the agreement a control sample of $B \rightarrow D^{*} l \nu$ decays are used. Finally the selection efficiencies for the various channels will be given.

\subsection{Data Set and Monte Carlo Samples}

The analysis uses $91.5 \mathrm{fb}^{-1}$ of data recorded between 1999 and 2002 by the $B A B A R$ detector. $81.9 \mathrm{fb}^{-1}$ of the data is so-called on-resonance data, taken at a centre 


\begin{tabular}{|c|c|}
\hline$e^{+} e^{-} \rightarrow$ & Cross Section (nb) \\
\hline$b \bar{b}$ & 1.05 \\
$c \bar{c}$ & 1.30 \\
$u \bar{u}$ & 1.39 \\
$d \bar{d}$ & 0.35 \\
\hline$\tau^{+} \tau^{-}$ & 0.94 \\
$\mu^{+} \mu^{-}$ & 1.16 \\
$e^{+} e^{-}$ & $\sim 40$ \\
\hline
\end{tabular}

Table 4.1: Production cross-sections at the $\Upsilon(4 S)$ resonance [6]. The $e^{+} e^{-}$cross-section given is the effective cross-section expected within the experimental acceptance.

of mass (CMS) energy equal to $10.58 \mathrm{GeV}$, the mass of the $\Upsilon(4 S)$ resonance. The remaining $9.6 \mathrm{fb}^{-1}$ is recorded at a CMS energy $40 \mathrm{MeV}$ lower, below the threshold for $B \bar{B}$ production. These off-resonance data are used to describe the events that proceed through quarks other than $b$ quarks, so-called continuum events. The cross sections adopted for the different processes at the $\Upsilon(4 S)$ resonance are given in Table 4.1.

Monte Carlo simulation is used to determine the efficiencies of the selections made during the analysis and to provide the shapes of various components used in the final fit. The Monte Carlo samples used are:

- 174 million $B^{0} \bar{B}^{0}$ generic decays,

- 14 million $B^{0} \bar{B}^{0}$ hard lepton generic decays,

- 174 million $B^{+} B^{-}$generic decays,

- 14 million $B^{+} B^{-}$hard lepton generic decays,

- 113 million $c \bar{c}$ decays,

- 180 million $u \bar{u}, d \bar{d}, s \bar{s}$ decays,

- $274,000 B^{0} \rightarrow \pi^{+} l^{-} \nu$ decays,

- $142,000 B^{-} \rightarrow \pi^{0} l^{-} \nu$ decays,

- $322,000 B^{0} \rightarrow \rho^{+} l^{-} \nu$ decays, 


\begin{tabular}{|cc|cc|}
\hline Decay Mode & Branching Fraction & Decay Mode & Branching Fraction \\
\hline$B^{0} \rightarrow a_{0}^{-} l \nu$ & $14.10^{-6}$ & $B^{+} \rightarrow \eta l \nu$ & $30.10^{-6}$ \\
$B^{0} \rightarrow a_{1}^{-} l \nu$ & $165.10^{-6}$ & $B^{+} \rightarrow \eta^{\prime} l \nu$ & $60.10^{-6}$ \\
$B^{0} \rightarrow a_{2}^{-} l \nu$ & $14.10^{-6}$ & $B^{+} \rightarrow a_{0}^{0} l \nu$ & $7.10^{-6}$ \\
$B \rightarrow b_{1}^{-} l \nu$ & $102.10^{-6}$ & $B^{+} \rightarrow a_{1}^{0} l \nu$ & $82.10^{-6}$ \\
& & $B^{+} \rightarrow a_{2}^{0} l \nu$ & $7.10^{-6}$ \\
& $B^{+} \rightarrow b_{1}^{0} l \nu$ & $48.10^{-6}$ \\
& $B^{+} \rightarrow f_{0}^{0} l \nu$ & $4.10^{-6}$ \\
& $B^{+} \rightarrow f_{0}^{\prime 0} l \nu$ & $4.10^{-6}$ \\
& $B^{+} \rightarrow f_{1}^{0} l \nu$ & $41.10^{-6}$ \\
& $B^{+} \rightarrow f_{1}^{\prime 0} l \nu$ & $41.10^{-6}$ \\
& & $B^{+} \rightarrow f_{2}^{0} l \nu$ & $4.10^{-6}$ \\
& & $B^{+} \rightarrow f_{2}^{\prime 0} l \nu$ & $4.10^{-6}$ \\
& & $B^{+} \rightarrow h_{1}^{0} l \nu$ & $24.10^{-6}$ \\
& & $B^{+} \rightarrow h_{1}^{\prime 0} l \nu$ & $24.10^{-6}$ \\
\hline Inclusive & & Inclusive & $616.10^{-6}$ \\
\hline
\end{tabular}

Table 4.2: The table shows the $B \rightarrow X_{u} l \nu$ branching fractions (excluding signal decays) used to generate the generic $B$ Monte Carlo. The inclusive fraction is purely non-resonant pion decays.

- $186,000 B^{-} \rightarrow \rho^{0} l \nu$ decays,

- $184,000 B^{-} \rightarrow \omega l \nu$ decays.

The $B \bar{B}$ hard lepton generic samples are filtered generic decays where at least one of the $B \mathrm{~s}$ is forced to decay semileptonically and the lepton must have a momentum greater than $1.0 \mathrm{GeV}$ in the CM frame. GEANT 4 [37] is used to provide a full simulation of the BABAR detector. The resonant $B \rightarrow X_{u} l \nu$ decays are modelled using the ISGW2 model [17]. The dominant background of $B \rightarrow X_{c} l \nu$ decays is also modelled using the ISGW2 model, except $B \rightarrow D^{*} l \nu$ for which HQET is used. Nonresonant semileptonic $b \rightarrow u l \nu$ decays are simulated using calculations by de Fazio and Neubert [38] and fragmented by JETSET [39]. All continuum hadronisation and decays are simulated using JETSET. Table 4.2 gives the $B \rightarrow X_{u} l \nu$ branching fractions assumed in generic Monte Carlo. Table 4.3 gives the branching fractions of the charmed semileptonic decays. The charmed values have been adjusted from those used to generate the Mone Carlo to the more up to date values found in [7].

In order to improve agreement between Monte Carlo simulated decays and data a series of corrections are applied. These are detailed in Section 4.11. 


\begin{tabular}{|cc|cc|}
\hline Decay Mode & Branching Fraction (\%) & Decay Mode & Branching Fraction (\%) \\
\hline$B^{0} \rightarrow D^{-} l \nu$ & 2.11 & $B^{+} \rightarrow D^{0} l \nu$ & 2.15 \\
$B^{0} \rightarrow D^{*-} l \nu$ & 4.6 & $B^{+} \rightarrow D^{* 0} l \nu$ & 5.3 \\
$B^{0} \rightarrow c l \nu$ & 3.5 & $B^{+} \rightarrow c l \nu$ & 3.05 \\
\hline Total & 10.2 & Total & 10.5 \\
\hline
\end{tabular}

Table 4.3: The table shows the $B \rightarrow X_{c} l \nu$ branching fractions. $B^{0} \rightarrow c l \nu$ includes all charmed modes other than those given

\subsection{Event Preselection}

For practical reasons, in order to reduce the amount of processing time and ntuple size required, some preselection is applied to the data. It is required that the event passes at least one of two criteria determined by the level 3 trigger, L3EmcOut or L3DchOut. These select events based upon clusters recorded in the EMC and tracks in the $\mathrm{DCH}$ respectively. They also provide some rejection of $e^{+} e^{-} \rightarrow e^{+} e^{-}$events. In addition it is required that there are more than 3 tracks recorded in the event, and that the second moment of the normalised Fox Wolfram moment $\left(R_{2}\right)[40]$ is less than 0.6. Finally it is required that there is at least one lepton with a CMS momentum greater than $1.3 \mathrm{GeV}$. To summarise the preselection criteria are:

- Number of tracks $>3$,

- trigger bit L3EmcOut or L3DchOut set,

- $\mathrm{R} 2<0.6$

- at least one lepton with a CMS momentum $>1.3 \mathrm{GeV}$.

\subsection{Track Selection}

$B A B A R$ has a standard set of criteria, of varying rigour, used to select tracks. The track selection used in this analysis is based on two levels of the standard selection. The loosest of these selections is commonly refered to as GoodTracksVeryLoose which requires that the following criteria are met: 


\begin{tabular}{|c|c|}
\hline Tracks rejected & Rejection criteria \\
\hline Loopers & $p_{t}<180 \mathrm{MeV}$ and $\Delta p_{t}<100 \mathrm{MeV}$ \\
& $\begin{array}{c}\text { Same sign }:|\Delta \phi|<220 \mathrm{mrad} \text { and }|\Delta \theta|<215 \mathrm{mrad} \\
\text { opposite sign }:|\Delta \phi|<190 \mathrm{mrad} \text { and }|\Delta \theta|<300 \mathrm{mrad}\end{array}$ \\
\hline Duplicate tracks & $p_{t}<350 \mathrm{MeV}$ and $\Delta p_{t}<100 \mathrm{MeV}$ \\
& $|\Delta \phi|<220 \mathrm{mrad}$ and $|\Delta \theta|<215 \mathrm{mrad}$ \\
\hline SVT only tracks & $p_{t}>350 \mathrm{MeV}$ and $N_{D C H}=0$ \\
\hline
\end{tabular}

Table 4.4: Additional criteria used to remove duplicate tracks.

- the momentum of the track must be less than $10 \mathrm{GeV}$;

- the distance of closest approach (DOCA) from the track to the beam spot must be less than $\pm 1.5 \mathrm{~cm}$ in the $\mathrm{x}-\mathrm{y}$ plane. The maximum DOCA along the $\mathrm{z}$ axis is $\pm 10 \mathrm{~cm}$.

The tighter standard selection will be refered to as GoodTrackLoose. In addition to the previous selection, tracks in this category also require:

- a minimum transverse momentum of $0.1 \mathrm{GeV}$;

- at least $12 \mathrm{DCH}$ hits.

In addition to these standard selections criteria are applied to remove specific cases of duplicate tracks and "loopers". Duplicate tracks occur when the DCH tracking algorithms split a single track into two separate tracks which share DCH hits. These are removed by cutting on tracks with very little angular separation. The track with the most DCH hits is retained as it is assumed to have the best resolution. "Loopers" are tracks with few or no DCH hits and little transverse momentum that loop back into the SVT and are reconstructed as a second track. In order to remove these tracks, cuts are made on the angular separation between tracks with low momentum transverse to the beam axis $\left(p_{t}\right)$. Finally tracks with $p_{t}>200 \mathrm{MeV}$ are removed if they have no hits in the $\mathrm{DCH}$. The additional track selection applied is summarised in Table 4.4. 


\subsection{Neutral Selection}

\subsubsection{Photons}

Photon candidates are local maxima (bumps) in the EMC which are not associated with any tracks in the event. Again, two levels of photon selection are used in this analysis, loose and tight. For the loose selection the following criteria are applied to bumps:

- The bump must have a lateral moment (LAT) less than 0.8. LAT is defined as

$$
L A T=\frac{\sum_{i=3}^{N} E_{i} r_{i}^{2}}{\sum_{i=3}^{N} E_{i} r_{i}^{2}+E_{1} r_{0}^{2}+E_{2} r_{0}^{2}}
$$

where $E_{i}$ is the energy deposited in crystal $i$, numbering them such that $E_{1}>$ $E_{2}>\ldots>E_{N}, r_{i}$ is the distance from the bump centroid to crystal $i$ and $r_{0}=5 \mathrm{~cm}$ (the average distance between two crystals). This is designed to be discriminate between electromagnetic and hadronic showers.

- The candidate must have an energy greater than $30 \mathrm{MeV}$. This helps to reject background photons.

For the tight selection the following are also required:

- the minimum energy cut is raised to $50 \mathrm{MeV}$.

- It is found that some bumps reconstructed in the EMC are consistent with deposits from tracks that have been missed by the track-cluster matching algorithm. In order to remove these clusters, which amount to a double counting of energy when constructing the missing momentum in the event, the angular distance between the track and the bump is examined. The cluster is removed if the angular distance to the nearest track in the $\theta$ direction is in the range $|\Delta \theta|<30 \mathrm{mrad}$ and for the $\phi$ direction in the range $-30<Q \Delta \phi<80 \mathrm{mrad}$, where $Q$ is the charge of the track. 


\subsection{2 $\quad \pi^{0}$ Reconstruction}

The $\pi^{0}$ candidates are constructed by combining two loose photon candidates using the primary vertex of the event as the origin. The invariant mass of the two photons is required to be in the range $115 \mathrm{MeV}<M(\gamma \gamma)<150 \mathrm{MeV}$. A mass constrained fit is applied.

\subsection{Lepton Selection}

Lepton candidates are selected from tracks passing the GoodTracksLoose criteria. The polar angle of the lepton candidate is restricted to $0.409<\theta_{\text {lab }}<2.372 \mathrm{rad}$.

\subsubsection{Electron Identification}

Electron candidates are then selected as follows:

- the ratio of the energy measured in the calorimeter to the momentum of the track $(E / p)$ must be in the range $0.89<E / p<1.20$;

- there must be more than 3 crystals in the EMC shower, and the shape of the shower (LAT and A42) must be consistent with being caused by an electron. Showers caused by hadrons have a different shape to those from electrons which deposit most of their energy in a relatively limited region;

- the energy loss of the particle in the $\mathrm{DCH}(d E / d x)$ is required to be consistent with that expected for an electron, $540<d E / d x<860$ (arbitrary units);

- a cut is placed on the azimuthal angular distance between the extrapolated position at which the track crosses the EMC and the actual position of the cluster $(\Delta \phi)$. Hadrons tend to shower further into the crystal than electrons and hence have a larger $\Delta \phi$; 
- the DIRC information must be consistent with being an electron. This is only used if more than 10 DIRC photons are observed;

- the candidate is required to have a CMS momentum greater than $1.3 \mathrm{GeV}$.

The efficiency of the electron selection criteria has been determined in a separate study using a control sample of $e^{+} e^{-} \rightarrow e^{+} e^{-} \gamma$ events. The rate of misidentification of hadrons as leptons is also measured using control samples. Pion samples are provided by $K_{S}^{0} \rightarrow \pi^{+} \pi^{-}$decays and 3-1-prong decays of $\tau$ pairs. A clean kaon sample is obtained via the decay $D^{*+} \rightarrow K^{-} \pi^{+} \pi_{\text {slow }}^{+}$. Protons are extracted from $\Lambda \rightarrow p \pi$. Further details of the electron selection and control samples can be found in [41].

\subsubsection{Muon Identification}

The criteria for muon selection are:

- the energy deposited in the EMC must be consistent with that from a minimum ionising particle, $0.05<E_{\text {cal }}<0.40 \mathrm{GeV}$;

- the candidate must have hits in at least 2 layers of the IFR;

- the candidate must traverse at least 2.2 interaction lengths of material and be within one interaction length of that expected for the muon hypothesis;

- it is required that a 3 rd order polynomial fit to the hit IFR strips gives a $\chi^{2} /$ d.o.f. $<3$. It is also required that the $\chi^{2} /$ d.o.f. of the clusters with respect to the extrapolated track be less than 5 ;

- the average multiplicity of hit strips in an IFR layer must be less than 8 and have spread less than 4 ;

- the continuity of the IFR cluster is defined as $T_{c}=N /(L-F+1)$, where $N$ is the number of layers with a hit, and $L$ and $F$ are the last and first layers 
with a hit. $T_{c}$ is expected to be equal to one for muons in an ideal detector. $T_{c}>0.3$ is required for tracks in the polar angle region $0.3<\theta<1.0 \mathrm{rad}$.

The efficiency of the muon selector is determined using a control sample of $e^{+} e^{-} \rightarrow$ $e^{+} e^{-} \mu^{+} \mu^{-}$events. Misidentification rates are obtained from the same control samples as for electrons. Further details of the muon selection and control samples can be found in [42].

\subsubsection{Fake Leptons}

The lepton selection criteria do not reject all hadrons. In order to determine the size and shape of the non-leptonic (fake) background a control sample is selected which passes all of the reconstruction and selection criteria except the lepton condition. Leptons are vetoed from this control sample by only selecting tracks that fail a looser lepton selection than previously described. The fake background is then determined by weighting the fake sample according to hadron misidentification rates measured using data control samples. The derivation of these weights are described below.

The total number of tracks which pass the loose lepton veto is

$$
\begin{aligned}
N_{\text {loose }}^{\text {tot }}= & \left(1-\epsilon_{e \rightarrow e}^{\text {loose }}\right)\left(1-\epsilon_{e \rightarrow \mu}^{\text {loose }}\right) N_{e}+\left(1-\epsilon_{\mu \rightarrow \mu}^{\text {loose }}\right)\left(1-\epsilon_{\mu \rightarrow e}^{\text {loose }}\right) N_{\mu} \\
& +\left(1-\epsilon_{h \rightarrow e}^{\text {loose }}\right)\left(1-\epsilon_{h \rightarrow \mu}^{\text {loose }}\right) N_{h} \\
\simeq & \left(1-\epsilon_{e \rightarrow e}^{\text {loose }}\right) N_{e}+\left(1-\epsilon_{\mu \rightarrow \mu}^{\text {loose }}\right) N_{\mu}+\left(1-\epsilon_{h \rightarrow e}^{\text {loose }}\right)\left(1-\epsilon_{h \rightarrow e \mu}^{\text {loose }}\right) N_{h}
\end{aligned}
$$

where $N_{x}$ is the total number of particles of each type $x$ and $\epsilon_{x \rightarrow y}$ is the probability of particle type $x$ passing the selection $y$. The approximation $\epsilon_{\mu \rightarrow e} \simeq \epsilon_{e \rightarrow \mu} \simeq 0$ is used. The equation can be further simplified by assuming that the selection is sufficiently loose that all of the leptons fail the selection cuts. We then get

$$
N_{\text {loose }}^{\text {tot }}=\left(1-\epsilon_{h \rightarrow e}^{\text {loose }}\right)\left(1-\epsilon_{h \rightarrow \mu}^{\text {loose }}\right) N_{h} .
$$

The number of tracks passing the tight selection $N_{\text {tight }}^{l}$ (the one actually used in the analysis) is

$$
N_{\text {tight }}^{l}=\epsilon_{l \rightarrow l}^{t i g h t} N_{l}+\epsilon_{h \rightarrow l}^{t i g h t} N_{h},
$$


where $l$ is either a electron or muon. The fake contributions $\left(N_{\text {fake }}^{l}\right)$ can then be determined from,

$$
\begin{aligned}
N_{\text {fake }}^{l} & =\epsilon_{h \rightarrow l}^{\text {tight }} N_{h} \\
& =\frac{\epsilon_{h \rightarrow l}^{\text {tight }}}{\left(1-\epsilon_{h \rightarrow e}^{\text {loose }}\right)\left(1-\epsilon_{h \rightarrow \mu}^{\text {loose }}\right)} N_{\text {loose }}^{\text {tot }} \\
& =w(p, \theta, \phi) N_{\text {loose }}^{\text {tot }}
\end{aligned}
$$

In the above,

$$
\epsilon_{h \rightarrow l}=f_{\pi} \epsilon_{\pi \rightarrow l}+f_{K} \epsilon_{K \rightarrow l}+f_{p} \epsilon_{p \rightarrow l}
$$

where $f_{h}$ is the percentage contribution of particle type $h$ to the total number of hadrons. This is determined from the Monte Carlo samples.

\subsection{Hadron Selection}

The five $B \rightarrow X_{u} l \nu$ modes reconstructed in this analysis are $X_{u}=\pi^{ \pm}, \pi^{0}, \rho^{ \pm}, \rho^{0}$ and $\omega$. This section covers the reconstruction of the $X_{u}$ mesons.

The tracks used to form the $\omega$ are selected using the GoodTracksVeryLoose criteria; for all other modes GoodTracksLoose is used. In addition it is required that none of the tracks pass the signal quality lepton selection, or are consistent with a charged kaon for the $\rho$ and $\omega$ channels. When the reconstructed mode has at least one charged track as well as the lepton a vertex fit is performed and a minimum $\chi^{2}$ probability of $0.1 \%$ is required. The hadron must have the opposite charge to the lepton (for charged hadrons).

\subsection{1 $\quad \mathrm{B} \rightarrow \pi \mathrm{l} \nu$}

In order to remove the generic charm $b \rightarrow c l \nu$ background it is required that the sum of the magnitude of the momentum of the lepton and pion in the CM frame, $p_{\pi}^{*}+p_{l}^{*}$, be greater than $3.1 \mathrm{GeV}$. One source of non-semileptonic background is 
from decays involving $J / \psi \rightarrow \mu^{+} \mu^{-}\left(e^{+} e^{-}\right)$. Here one of the legs fails the lepton veto and acts as the pion, and the other is selected as the lepton. In order to remove this background the mass of the hadron and lepton candidate is calculated using the lepton mass hypothesis for both tracks and the candidate rejected if the mass falls within $\pm 25 \mathrm{MeV}$ of the $J / \psi$ mass.

\subsection{2 $\quad \mathrm{B} \rightarrow \rho \mathrm{l} \nu$}

The invariant mass of the $\pi \pi$ candidate is required to be within the range $0.65<$ $M(\pi \pi)<0.85 \mathrm{GeV} . p_{l}^{*}>2.0 \mathrm{GeV}$ is required. This severely restricts the amount of phase space available to the decay, but is necessary in order to suppress the huge background from $b \rightarrow c l \nu$ decays in this mode. The additional constraint $0.25 p_{l}^{*}+p_{\rho}^{*}>1.375 \mathrm{GeV}$ is found to provide additional suppression of both $b \rightarrow c l \nu$ and continuum decays. In order to reduce combinatorial background in this decay mode the higher momentum pion is required to have $p_{\text {max }}^{*}>0.4 \mathrm{GeV}$ and the lower momentum pion to have $p_{\min }^{*}>0.2 \mathrm{GeV}$. As in the case of $B \rightarrow \pi l \nu$ decays, if a $J / \psi$ candidate can be made, the candidate is rejected if it has a mass within $\pm 25 \mathrm{MeV}$ of the $J / \psi$ mass.

\subsection{3 $\quad \mathrm{B} \rightarrow \omega \mathrm{l} \nu$}

The $\omega$ is reconstructed in the mode $\omega \rightarrow \pi^{+} \pi^{-} \pi^{0}$, which accounts for $89.1 \%$ of all $\omega$ decays [7]. The invariant mass of the $\pi \pi \pi$ candidate is required to be within the range $765<M(\pi \pi \pi)<805 \mathrm{MeV}$. The lepton momentum is restricted to $p_{l}^{*}>2.0 \mathrm{GeV}$ and hadron momentum to $p_{\omega}^{*}>0.8 \mathrm{GeV}$. To reduce combinatorial background it is required that the Dalitz Amplitude $\propto\left|\mathbf{p}_{\pi^{+}}^{*} \times \mathbf{p}_{\pi^{-}}^{*}\right|[43]$ be greater than half its maximum value (see Fig. 4.1). It is also required that $p_{\pi^{0}}^{*}>0.2 \mathrm{GeV}$. As with the previous modes any possible $J / \psi$ candidates must not have an invariant mass within $\pm 25 \mathrm{MeV}$ of the $J / \psi$ mass. 


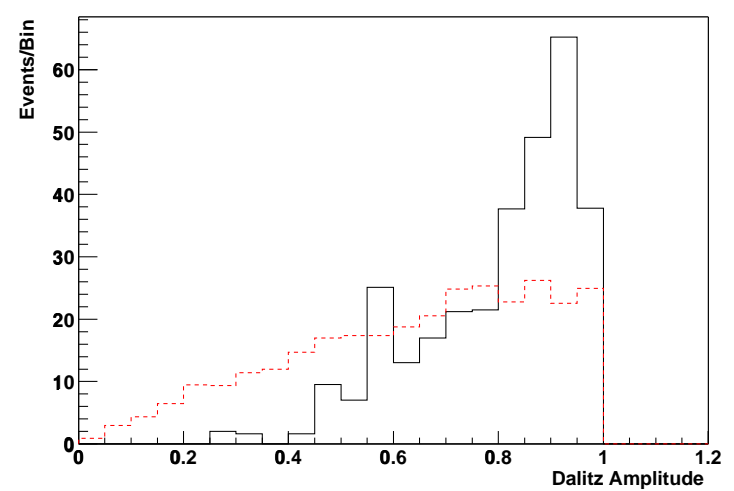

Figure 4.1: The Dalitz Amplitude of $B^{+} \rightarrow \omega l^{+} \nu$ mode. The true MC $B^{+} \rightarrow \omega l^{+} \nu$ decays are shown in black and the combinatoric generic $B \bar{B}$ background decays in dashed red.

\subsection{Neutrino Reconstruction}

This analysis attempts to make use of the full kinematics of the event by determining the momentum of the neutrino in the event. The missing four momentum in the event is given by

$$
\begin{aligned}
E_{\text {miss }} & =E_{\text {beams }}-\sum_{i} E_{i} \\
\mathbf{p}_{\text {miss }} & =\mathbf{p}_{\text {beams }}-\sum_{i} \mathbf{p}_{i}
\end{aligned}
$$

where $i$ runs over all tracks and clusters in the event. This determination is obviously affected by undetected particles in the event. These can either be charged particle/photons which fall outside the tracking region (the detector is only $\sim 90 \%$ hermetic) or neutral particles such as extra neutrinos or $K_{L}^{0}$ mesons. Each particle in the event is given the pion mass hypothesis, hence the reconstructed energy will be shifted from the true neutrino energy for events which include particles other than pions. To reflect the greater resolution on $\mathbf{p}_{\text {miss }}$ the four momentum of the neutrino is set to $p_{\nu}=\left\{\left|\mathbf{p}_{\text {miss }}\right|, \mathbf{p}_{\text {miss }}\right\}$ after neutrino quality cuts. 


\subsection{Selection Criteria}

The selection criteria applied in this analysis can generally be broken down into three distinct categories.

1. Selection designed to reduce the background from continuum decays which generally makes use of the more jet-like topology of these decays as compared to $B$ meson.

2. There are cuts which make use of the kinematics of the signal decays to reduce the background from other semileptonic decays including the dominant $b \rightarrow$ $c l \nu$ background, "feeddown" from higher $b \rightarrow u l \nu$ resonances and non-resonant semileptonic decays.

3. Finally there are cuts to improve the resolution on the momentum of the neutrino. These help to suppress background, especially from continuum decays, and are required to improve the resolution on the variables used to fit the sample.

\subsubsection{Event Shape Variables}

This set of variables is used primarily to suppress background from continuum decays. Continuum decays present a large background to this analysis which is particularly difficult to quantify because of the limited statistics of off-resonance data available at $B A B A R$. Some of this selection is just a tightening of preselection cuts already applied.

- The criterion on the multiplicity of the event is tightened to number of charged tracks $N_{c h}>4$, or $N_{c h} \geq 4$ and $N_{\gamma} \geq 2$, where $N_{\gamma}$ is the number of photons in the event.

- The cut on the normalised second moment of the Fox-Wolfram moment is set at $R_{2}<0.4$. 
- A combined cut is made on the second Legendre moment $L_{2}$ and cosine of the angle between the thrust axis of the combined hadron and lepton candidate (the $\mathrm{Y}$ candidate) and the rest of the event, $\cos \theta_{\text {Thrust }}$. Both of these variables exploit the fact that continuum events have a more jet-like topology than $B$ meson decays. To calculate $\cos \theta_{\text {Thrust }}$, the tracks in the event are separated into those coming from the $\mathrm{Y}$ candidate and those originating from the rest of the event. The thrust axis is defined as the direction that maximises the sum of the longitudinal momentum magnitude of the tracks. For continuum events $\left|\cos \theta_{\text {Thrust }}\right|$ is strongly peaked toward 1 as the jets in the event are generally back-to-back.

The Legendre mononomials are a set of momentum-weighted sums of the rest of the tracks in the event (i.e. not including the $\mathrm{Y}$ candidate) defined as

$$
L_{j}=\sum_{i}^{\text {r.o.e. }} p_{i} \times\left|\cos \theta_{i}\right|^{j}
$$

The first mononomial $L_{0}$ is closely related to the missing mass measurement of the event and hence highly correlated to the signal variables. This analysis uses the second Legendre monomial $L_{2}=\sum_{i}^{\text {r.o.e. }} p_{i} \times \cos ^{2} \theta_{i}$ which shows no obvious correlation with the signal variables. The two dimensional cut

$$
L_{2}+14.28 \cos \theta_{\text {Thrust }} \leq 14.28, \quad L_{2}-1.875 \cos \theta_{\text {Thrust }} \leq 1.0
$$

is used.

\subsubsection{Kinematic Selection}

The kinematics of the decay $B \rightarrow X_{u} l \nu$ decay are illustrated in Fig. 4.2.

Using the constraint that the neutrino four momentum satisfies $p_{\nu}^{2}=0=\left(p_{B}-p_{X_{u}}-p_{l}\right)^{2}=$ $\left(p_{B}-p_{Y}\right)^{2}$ and that the energy of the $B\left(E_{B}\right)$ is equal to the beam energy $E_{\text {beam }}$, four momentum conservation leads to

$$
\cos \theta_{B Y}=\frac{2 E_{B} E_{Y}-m_{B}^{2}-m_{Y}^{2}}{2\left|\mathbf{p}_{\mathbf{B}}\right|\left|\mathbf{p}_{\mathbf{Y}}\right|}
$$




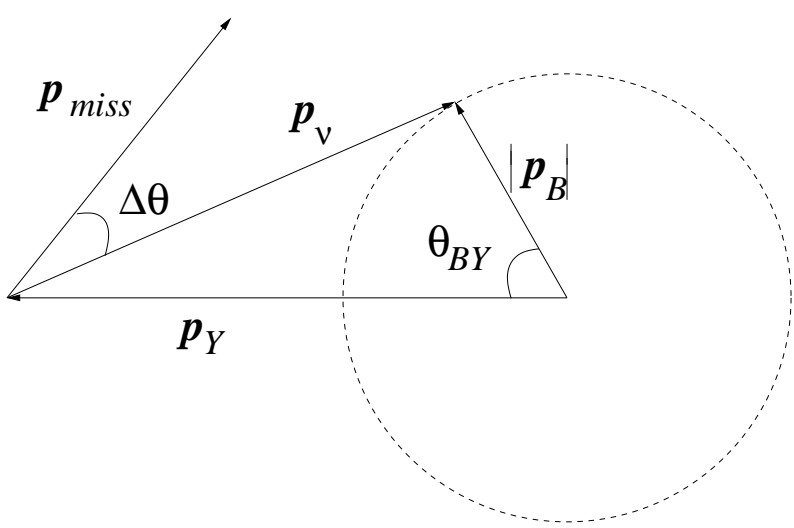

Figure 4.2: Kinematics of $B \rightarrow X_{u} l \nu$ decays. $Y$ represents the combined $X_{u} l$ system.

For signal decays the value of $\cos \theta_{B Y}$ should lie between -1 and 1 . There is no such requirement for background decays. The cut $-1.1<\cos \theta_{B Y}<1.1$ is made. Note the reason that we do not cut at 1.0 is to account for bad resolution on the tracks and for bremsstrahlung. The muon is less likely to bremsstrahlung than an electron, meaning this cut performs better for muons than electrons as illustrated in Fig. 4.3.
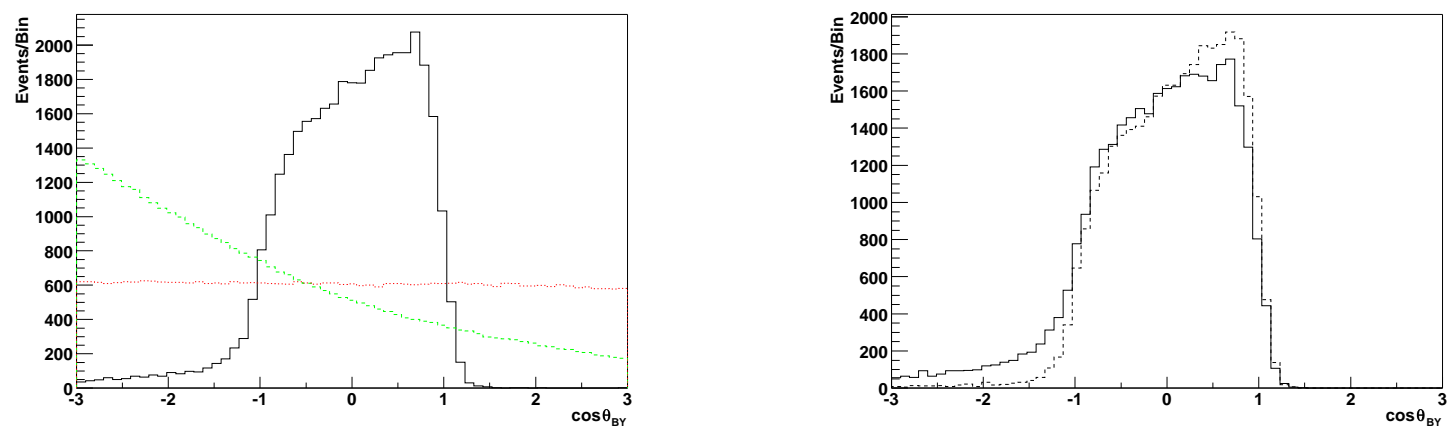

Figure 4.3: Distribution of $\cos \theta_{B Y}$. The left plot shows the distribution of $\cos \theta_{B Y}$ decays reconstructed in the $B^{0} \rightarrow \pi^{-} l^{+} \nu$ mode from Monte Carlo samples. The correctly reconstructed signal decays are shown in black, generic $B^{0}$ decays in green and off-resonance in red. The right plot shows the $\cos \theta_{B Y}$ distributions for correctly reconstructed signal decays separated into electrons (solid) and muons (dashed).

It is also possible to use the decay kinematics to determine the angle between the measured missing momentum and the actual neutrino momentum, up to an azimuthal ambiguity. The angle can be written as

$$
\cos \Delta \theta=\frac{\mathbf{p}_{\nu} \cdot \mathbf{p}_{\text {miss }}}{\left|\mathbf{p}_{\nu}\right|\left|\mathbf{p}_{\text {miss }}\right|}=\frac{\left(\mathbf{p}_{B}-\mathbf{p}_{Y}\right) \cdot \mathbf{p}_{\text {miss }}}{\left|\mathbf{p}_{\nu}\right|\left|\mathbf{p}_{\text {miss }}\right|}
$$


where

$$
\begin{aligned}
& \mathbf{p}_{B} \cdot \mathbf{p}_{\text {miss }}=\left|\mathbf{p}_{B}\right|\left|\mathbf{p}_{\text {miss }}\right| \cos \theta_{B, \text { miss }} \\
& \mathbf{p}_{Y} \cdot \mathbf{p}_{\text {miss }}=\left|\mathbf{p}_{Y}\right|\left|\mathbf{p}_{\text {miss }}\right| \cos \theta_{Y, \text { miss }}
\end{aligned}
$$

Although it is not possible to determine the angle exactly, it will be a minimum when $\mathbf{p}_{Y}, \mathbf{p}_{B}$ and $\mathbf{p}_{\text {miss }}$ fall in the same plane. When this is the case,

$$
\begin{aligned}
\cos \theta_{B, m i s s} & =\cos \left(\theta_{B, Y}-\theta_{Y, m i s s}\right) \\
& =\cos \theta_{B Y} \cos \theta_{Y, m i s s}+\sin \theta_{B, Y} \sin \theta_{Y, m i s s}
\end{aligned}
$$

Hence it is possible to determine the minimum angle between the true neutrino momentum $\mathbf{p}_{\nu}$ and $\mathbf{p}_{\text {miss }}$. Figure 4.4 shows the distribution of $\Delta \theta$ for signal $B^{0} \rightarrow$ $\pi^{-} \ell^{+} \nu$ decays and generic $B \bar{B}$ background after all other selection is applied. The cut $|\Delta \theta|<0.6 \mathrm{rad}$ is made.

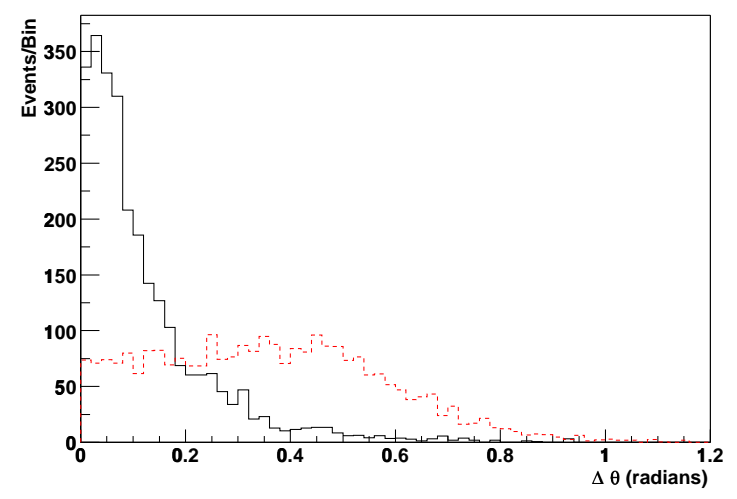

Figure 4.4: $|\Delta \theta|$ for events reconstructed in the $B^{0} \rightarrow \pi^{-} \ell^{+} \nu$ mode after all other selection has been applied. The true MC $B^{0} \rightarrow \pi^{-} \ell^{+} \nu$ decays are shown in black and generic $B \bar{B}$ background decays in dashed red.

\subsubsection{Neutrino Selection}

For a well reconstructed neutrino it is expected that $p_{m i s s}^{2}=E_{m i s s}^{2}-\mathbf{p}_{m i s s}^{2}=p_{\nu}^{2}=0$. This provides a powerful constraint and a useful tool for removing background. As 
the resolution on the missing mass squared $\left(M_{m i s s}^{2}\right)$ is dominated by the resolution on $E_{\text {miss }}$ the cut $\left|M_{\text {miss }}^{2} / 2 E_{\text {miss }}\right|<400 \mathrm{MeV}$ is made. A non-zero event charge $(Q)$ is a sign that charged tracks have been missed from the event and hence the reconstructed $p_{m i s s}$ will not correspond to the momentum of the neutrino. The cut $|Q| \leq 1$ is made. The BABAR detector has a hole in the forward direction. Particles that are produced with a direction close to the beam pipe will escape reconstruction and mimic a neutrino. To remove such cases we cut on the direction of the missing momentum and remove events with $0.25<\theta<2.90 \mathrm{rad}$. As well as cutting on the direction of the missing momentum it also found to be advantageous to remove events with $\left|\mathbf{p}_{\text {miss }}\right|<1.0 \mathrm{GeV}$ or $\left|\mathbf{p}_{\text {miss }}\right|>-2.0 \theta_{\text {miss }}+3.0 \mathrm{GeV}$ (where $\theta_{\text {miss }}$ is the direction of the neutrino in $\theta$ ). This selection is very efficient at removing continuum events.

\subsection{Fit Variables}

In this analysis we choose to fit in two variables, $\Delta E$ and the "beam energy substituted mass" $m_{E S}$, which exploit the kinematics of $\Upsilon(4 S) \rightarrow B \bar{B}$ events and show minimal correlation [44]. $\Delta E$ uses to fact that both decay products of the $\Upsilon(4 S)$ should have the same mass, the mass of the $B$. If $q_{\text {beams }}$ is the four momentum of the beam and $q_{B_{1}}, q_{B_{2}}$ the momenta of the two $B$ mesons, energy and momentum conservation give

$$
q_{\text {beams }}=q_{B_{1}}+q_{B_{2}} .
$$

Now calculating the difference in mass of the two $B$ candidates

$$
\begin{aligned}
\Delta q^{2} & =q_{B_{1}}^{2}-q_{B_{2}}^{2} \\
& =2 q_{\text {beams }} q_{B_{1}}-s
\end{aligned}
$$

where $s$ is the centre of mass energy squared. $\Delta E$ is defined as

$$
\Delta E=\frac{\Delta q^{2}}{2 s^{\frac{1}{2}}}=\frac{\left(q_{B_{1}} \cdot q_{\text {beams }}-s\right)}{2 s^{\frac{1}{2}}},
$$


which is the invariant form of the $\Delta E$ traditionally used by CLEO and ARGUS [44] usually written in the centre of mass frame

$$
\Delta E=E_{B_{1}}^{*}-E_{\text {beams }}^{*},
$$

where asterisk superscripts denote centre of mass frame variables.

The second variable used is $m_{E S}$ which is equal to $\sqrt{q_{B_{1}}^{2}}$, evaluated under the constraint that both $B$ mesons have the same mass, i.e. $\Delta q^{2}=0$ (equivalent to $\Delta E=0)$.

$$
\begin{aligned}
q_{B_{1}}^{2} & =E_{B_{1}}^{2}-\mathbf{p}_{B_{1}}^{2} \\
2 q_{\text {beams }} q_{B_{1}}-s & =0
\end{aligned}
$$

These two equations lead to

$$
m_{E S}=\sqrt{q_{B_{1}}^{2}}=\sqrt{\frac{\left(s / 2+\mathbf{p}_{B_{1}} \cdot \mathbf{p}_{\text {beams }}\right)^{2}}{E_{\text {beams }}^{2}}-\mathbf{p}_{B_{1}}^{2}} .
$$

In the centre of mass frame $m_{E S}$ reduces to the form

$$
m_{E S}=\sqrt{E_{\text {beams }}^{* 2}-\mathbf{p}_{B_{1}}^{* 2}} .
$$

In this frame the reason for the name "energy substituted mass" is obvious, $m_{E S}$ is the mass of the candidate $B$ meson calculated with $E_{\text {beams }}^{*}$ substituted for $E_{B_{1}}^{*}$. The resolution on $E_{\text {beams }}$ is much greater than that on $E_{B 1}$.

The region $-0.95<\Delta E<0.95 \mathrm{GeV}, 5.095<m_{E S}<5.305 \mathrm{GeV}$ is defined as the fit plane, the region which is fitted to extract the branching fractions. To get a better idea of the signal to noise ratio achieved a signal region is defined as $-0.15<\Delta E<0.25 \mathrm{GeV}$ and $m_{E S}>5.255 \mathrm{GeV}$.

\subsection{Monte Carlo Corrections}

The Monte Carlo simulation used is not perfectly tuned to the data and corrections are required to improve agreement. Most corrections in this analysis are performed by weighting the Monte Carlo events. The individual corrections applied are discussed in this section. 


\subsubsection{Charged PID Efficiency Correction}

The analysis uses particle identification (PID) to select leptons and to veto kaons. The PID efficiencies are determined as a function of lab momentum, polar angle and azimuthal angle using control samples. For electrons a sample of $e^{+} e^{-} \rightarrow e^{+} e^{-} \gamma$ is used and $e^{+} e^{-} \rightarrow e^{+} e^{-} \mu^{+} \mu^{-}$is used for muons. The efficiency on the control samples is determined for both data and Monte Carlo events. The ratio is then used to assign a weight to each lepton selected in Monte Carlo simulation.

\subsubsection{Neutrals Efficiency and Resolution Corrections}

The derivation and implementation of the neutrals corrections are described in detail in [45]. A smearing of up to $3 \%$ is applied to Monte Carlo photons of less than $1 \mathrm{GeV}$. The neutrals energy scale of Monte Carlo events produced with Run2 data conditions is also shifted by a correction factor. $\pi^{0}$ mesons are reconstructed with a better efficiency in Monte Carlo events than in the data. In order to get the efficiencies to match a weight is assigned to each $\pi^{0}$ candidate in the Monte Carlo event. The correction factors used here have been derived in a separate study of $e^{+} e^{-} \rightarrow \tau^{+} \tau^{-}$decays [46]. One of the $\tau$ leptons decays to a lepton and two neutrinos and the other to $h \pi^{0}\left(\pi^{0}\right) \nu$ where $h$ is either a charged pion or kaon.

\subsubsection{Tracking Efficiency Correction}

The tracking efficiency for charged tracks has been determined in a separate study [47]. One way to determine the tracking efficiency is to select events whose physics require a certain number of tracks and measure how often the required number of tracks are found. $e^{+} e^{-} \rightarrow \tau^{+} \tau^{-}$events where one $\tau$ decays leptonically and the other to three pions and an arbitrary number of neutral particles (a so-called 1-3 topology) are good candidates. Another method is to compare the number of tracks constructed in the SVT to the number reconstructed in the DCH separately. Tables are produced which contain the ratio for tracking efficiency for Monte Carlo events 
compared to data, binned in momentum, azimuthal and polar angle. These tables are used to assign a weight to each track in hadron and lepton candidate. Monte Carlo samples and data are found to be in agreement at around the $1 \%$ level.

\subsection{Data/MC Comparisons}

Since the expected signal sample is rather small, it will be difficult to use it to assess the accuracy of the MC simulation, both in terms of resolution and yield. We therefore select $B^{0} \rightarrow D^{*-} \ell^{+} \nu$ decays with $D^{*-} \rightarrow \overline{D^{0}} \pi^{-}$and use them as a control sample for this purpose. These decays exceed the rate for $B^{+} \rightarrow \rho^{0} \ell^{+} \nu$ by a factor of 30 (taking into account the $\overline{D^{0}}$ branching fractions, but not their detection efficiencies). They resemble the signal events in topology, and have similar spectra for the lepton and neutrino. The presence of the low energy pion impacts the detection efficiency for this sample, but not the reconstruction of the missing momentum. The $\overline{D^{0}}$ mesons are reconstructed through the decay modes: $\overline{D^{0}} \rightarrow$ $K^{+} \pi^{-}, K^{+} \pi^{+} \pi^{-} \pi^{-}, K^{+} \pi^{-} \pi^{0}$.

\subsubsection{Selection of $\mathrm{B}^{0} \rightarrow \mathrm{D}^{*-} \ell^{+} \nu$ Decays}

The following criteria are applied to select the $D^{*-} \ell^{+} \nu$ final state, starting with the BToDstarnuloose tag bit:

- the charged tracks from the $\overline{D^{0}}$ are selected using the GoodTracksLoose criteria. The slow pion is selected using the GoodTracksVeryLoose criteria;

- for $K^{+} \pi^{-}$decays, the $K^{+}$must satisfy a pion veto. For the other decay modes, the $K^{+}$is required to be consistent with a kaon;

- the effective mass of the $\overline{D^{0}}$ decay products should be within $17 \mathrm{MeV}(34 \mathrm{MeV}$ for $K^{+} \pi^{-} \pi^{0}$ ) of the nominal $D^{0}$ mass and the vertex fit probability should exceed $0.1 \%$; 
- the magnitude of the total $\left(p^{\pi}\right)$ and transverse $\left(p_{t}^{\pi}\right)$ momentum of the slow $\pi^{-}$ should fulfil the condition $p_{t}^{\pi}>0.050 \mathrm{GeV}$ and $p^{\pi}<0.45 \mathrm{GeV}$;

- to reduce backgrounds from continuum and combinatorics, the momentum of the $D^{*-}$ in the CM frame is restricted to $0.5<p_{D^{*}}^{*}<2.5 \mathrm{GeV}$;

- the angle between the $D^{*-}$ and the charged lepton momenta should satisfy $\cos \left(D^{*}, \ell\right)<0$, and $D^{*-}$ and $\ell^{+}$should be consistent with a common vertex with probability $>1.0 \%$;

- the mass difference between the $D^{*-}$ and the $D$ mesons $(\Delta m)$ is restricted to $0.1432<\Delta m<0.1478 \mathrm{MeV}$.

The estimated efficiency for $D^{0} \rightarrow K^{+} \pi^{-}$using this selection is typically $12.5 \%$, the branching fraction for these decays is $0.24 \%$, thus we expect 310 events $/ \mathrm{fb}^{-1}$.

The same selection criteria as for the signal $B \rightarrow X_{u} \ell \nu$ events is applied.

\subsubsection{Resolution in Missing Momentum and Energy}

The separation of the signal $B \rightarrow X_{u} \ell \nu$ events from the various background sources relies heavily on $\mathrm{MC}$ simulation, thus it is very important that these simulations describe the data well. The $\overline{D^{0}} \rightarrow K^{+} \pi^{-}$control sample, with its high purity, is particularly useful to compare quantities used in the reconstruction of the missing momentum from the whole event.

Figure 4.5 shows the MC derived resolutions for the missing neutrino for events with a reconstructed $B^{0} \rightarrow D^{*-} \ell^{+} \nu$ and $\overline{D^{0}} \rightarrow K^{+} \pi^{-}$decay, after all selection cuts. The error distributions for the momentum, $\left|\mathbf{p}_{\text {miss }}\right|-\left|\mathbf{p}_{\nu}\right|$, the energy, $E_{\text {miss }}-E_{\nu}$, and angle, $\theta_{\text {miss }}-\theta_{\nu}$, have long tails. Large differences between the reconstructed and the true values for the neutrino are due to lost or poorly reconstructed tracks and photons or undetectable particles, like additional neutrinos or $K_{L}$ from the second $B$ decay in the event. 

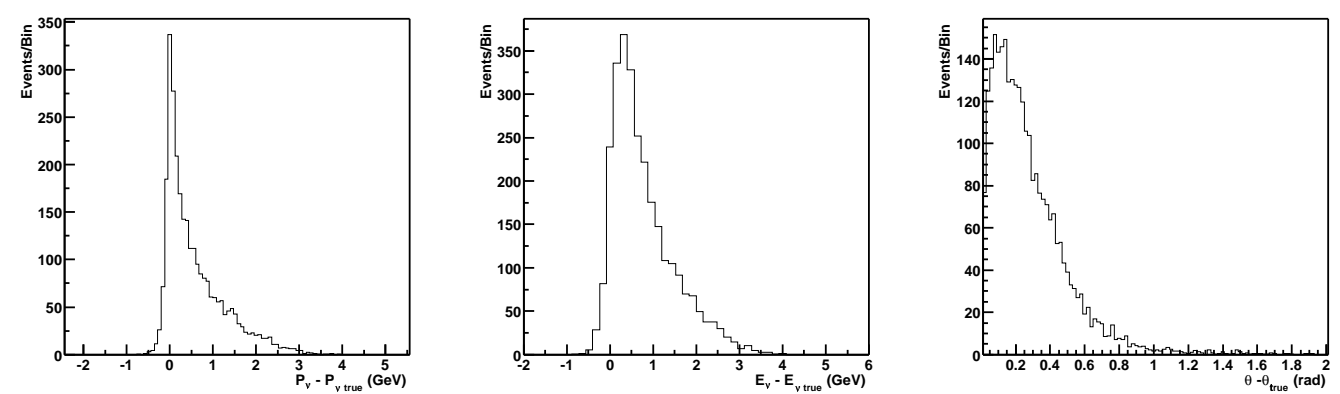

Figure 4.5: MC predicted resolutions of the momentum magnitude (left), energy (right), direction (right) of the missing particle for the $\overline{D^{0}} \rightarrow K^{+} \pi^{-}$subsample of the control sample.

Figures 4.6 - 4.9 show $\mathrm{MC} /$ data comparisons for the control sample with the $\overline{D^{0}} \rightarrow$ $K^{+} \pi^{-}$decay mode, before and after the selection of the semileptonic decay. The agreement is reasonably good. There are, however, noticeable differences in the square of the missing mass, the lepton momentum, and the energy of the low momentum clusters reconstructed in the event. Some of these differences might be caused by inaccurate form factor modelling of the $B^{0} \rightarrow D^{*-} \ell^{+} \nu$, others by inaccuracies in the detector simulation.

\subsubsection{Efficiency Studies}

Tables 4.5 to 4.10 list the number of $D^{*-} \ell^{+} \nu$ decays passing the cuts, both for data and MC. The tables are presented separately for electrons and muons, and the three different $\overline{D^{0}}$ decay modes.

Inaccuracies in the Monte Carlo simulation lead to discrepancies in the selection efficiencies between Monte Carlo and data. For the present analysis the most severe discrepancy is associated with the reconstruction of the missing momentum of the event, specifcally the selection made using $M_{m i s s}^{2} / 2 E_{m i s s}$. For events with correctly reconstructed signal combinations (as is mostly the case in the control sample; the $\overline{D^{0}} \rightarrow K^{+} \pi^{-}$mode has a purity of $\approx 89 \%$ ) the distribution of the missing momentum should be similar for the control sample and the charmless decays. The ratio $\epsilon_{\text {data }} / \epsilon_{M C}$ measured using the control sample is used to correct the Monte Carlo efficiency. From Tables 4.5 to $4.10, \epsilon_{\text {data }} / \epsilon_{M C}=1.082 \pm 0.012$ 

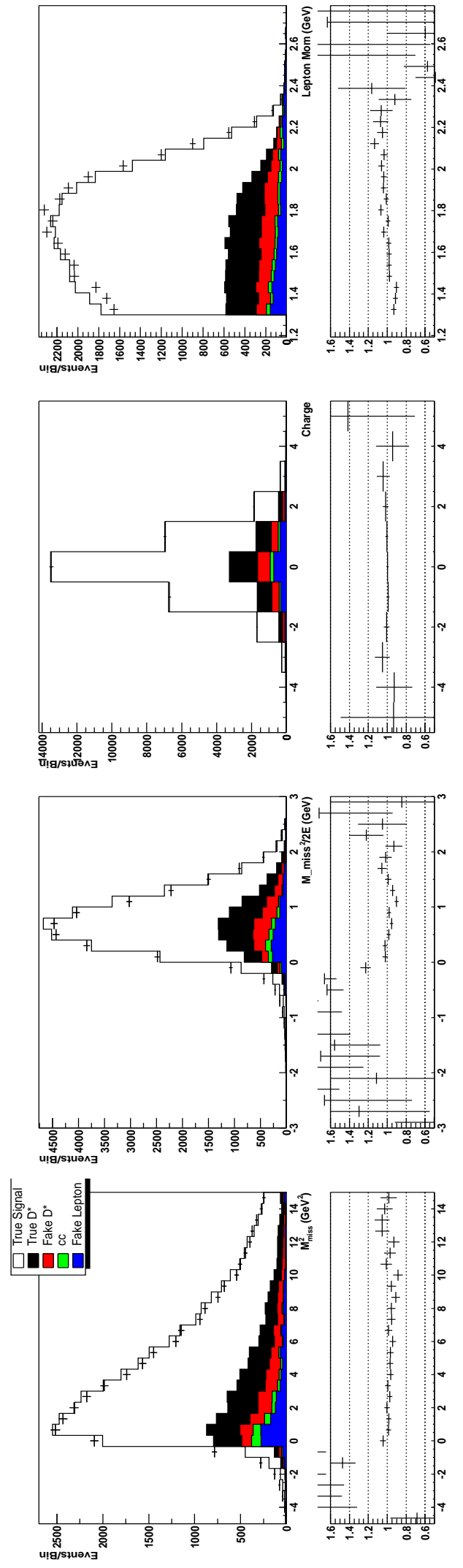
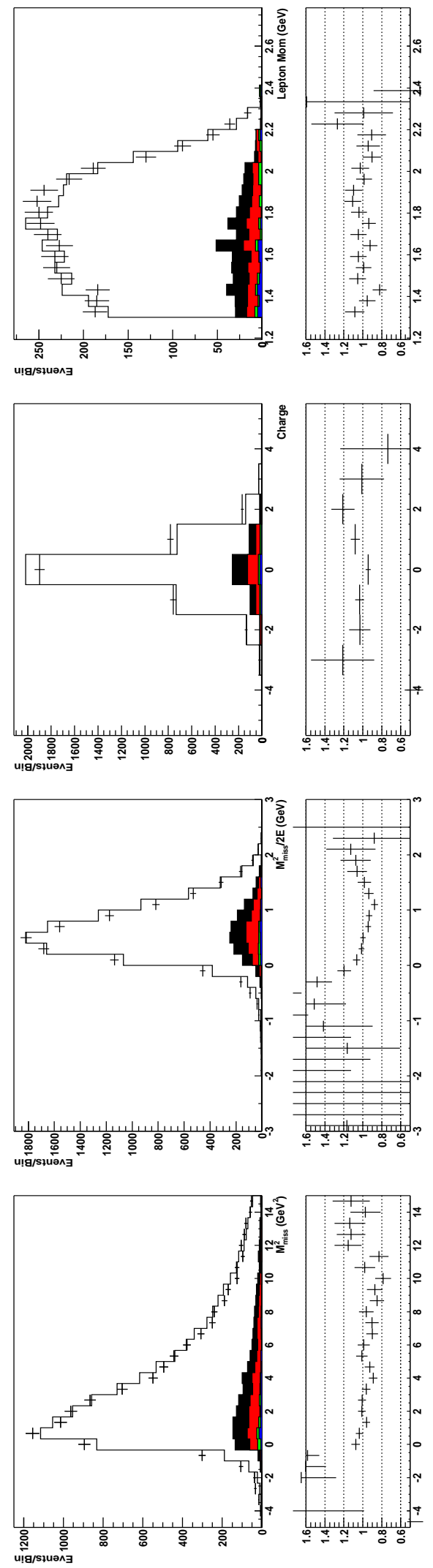

구유

.

进的政

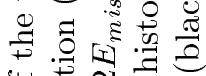

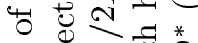

은 $\frac{0}{d}$ o

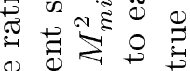

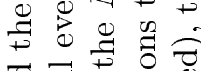

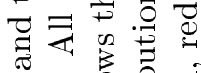

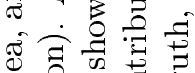

ฮั?

\&

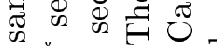

苟导的

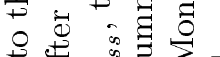

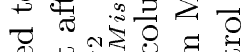

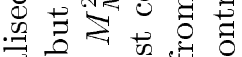

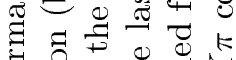

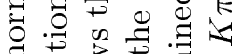

घ

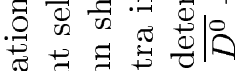

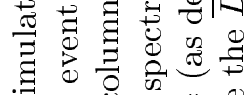

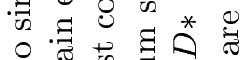

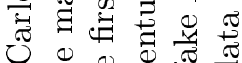

U

苛芯

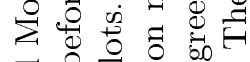

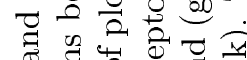

ส

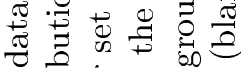

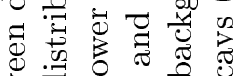

:

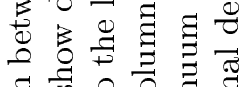

б

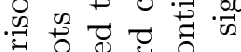

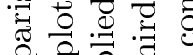

घ्चु

웡

击

एँ

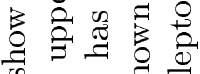

क 0 要

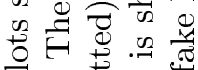

2. $\dot{2} \div$

\& $\frac{\pi}{2}$ है

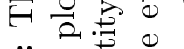

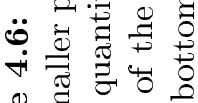

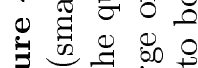

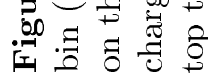



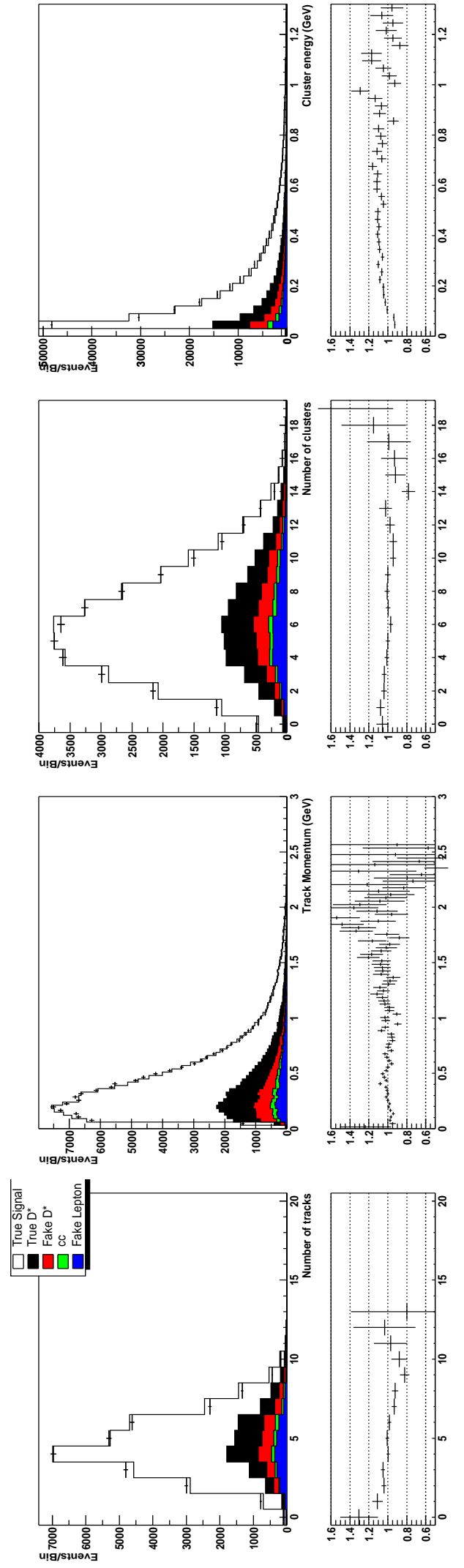

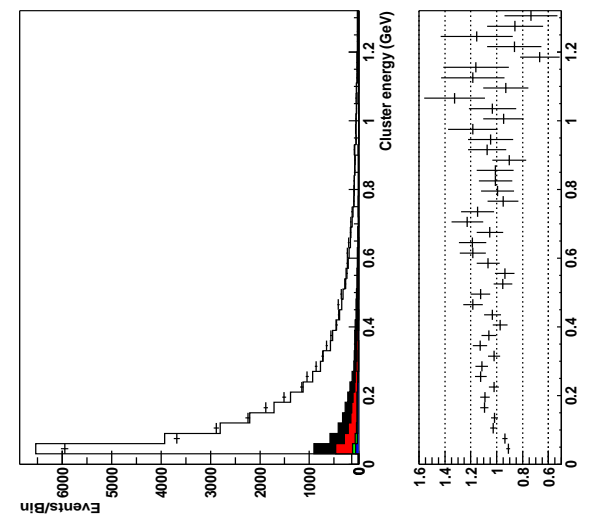

궁

I 言焉

运焉焉

㟧

苂䓯

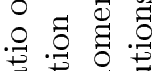

T유

里焉焉

눙ㅁㅇㅇ

ส

đึ

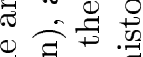

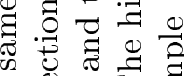

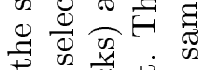
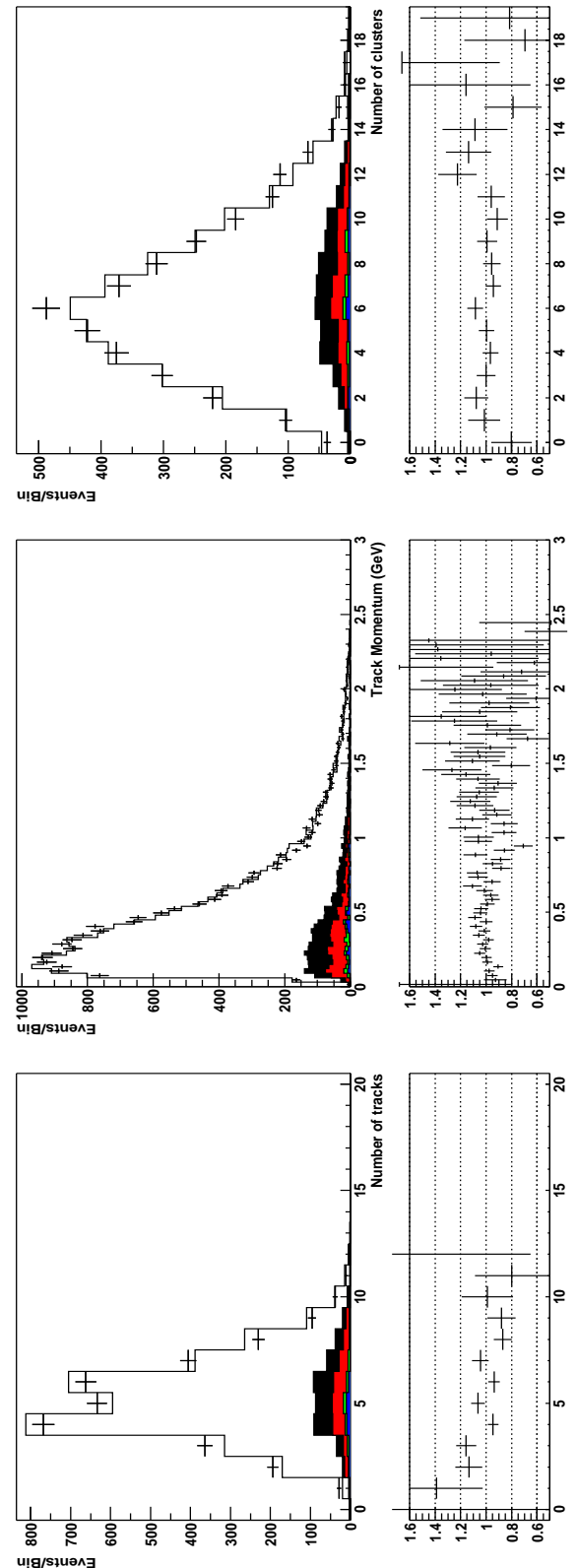

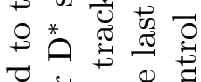

峁

बี

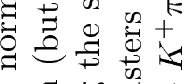

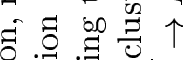

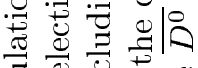

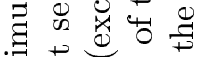

舟矛施。

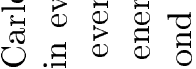

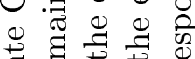

总

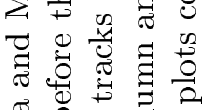

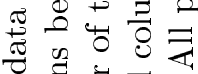

둥.

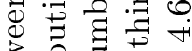

过苛寻.

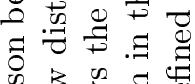

员言管言焉

苗 曹 $\frac{0}{5}$

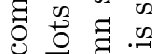

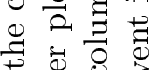

ॠ

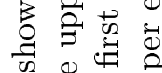

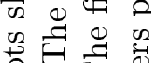

质焉

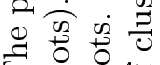

F

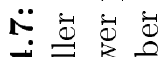

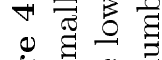

局手

我贯过 

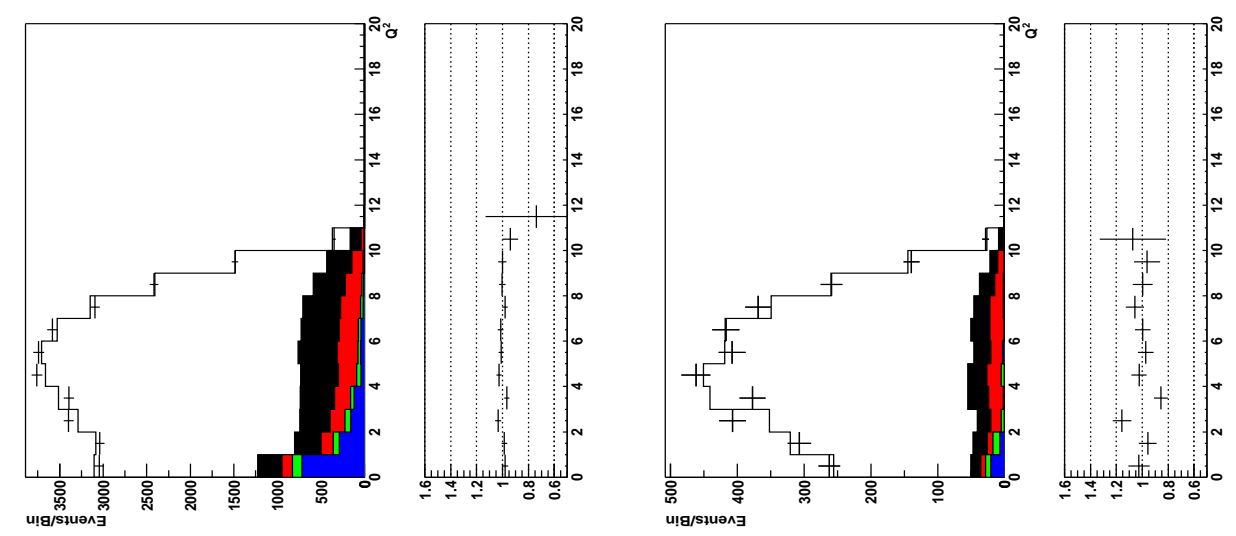

疋
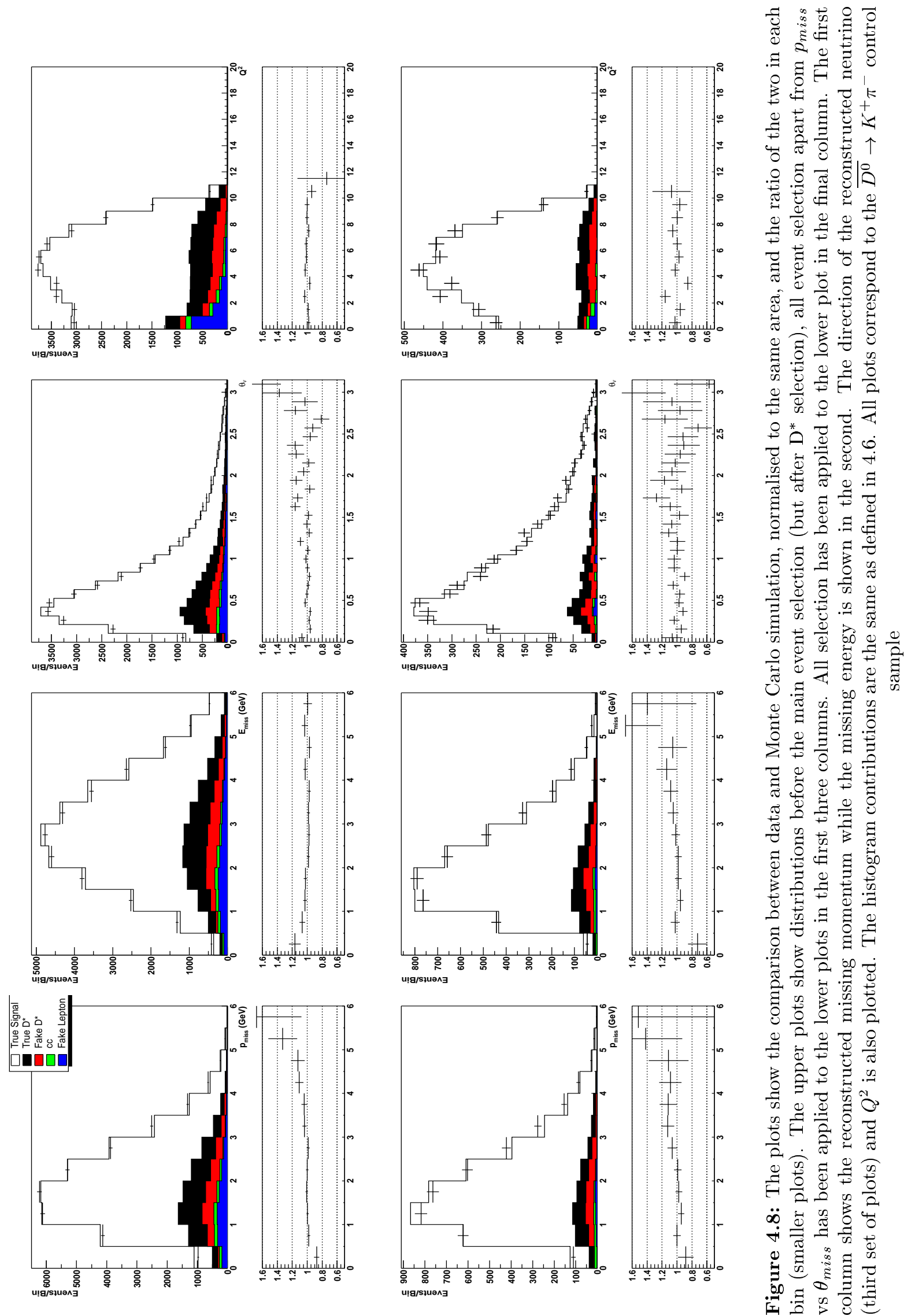

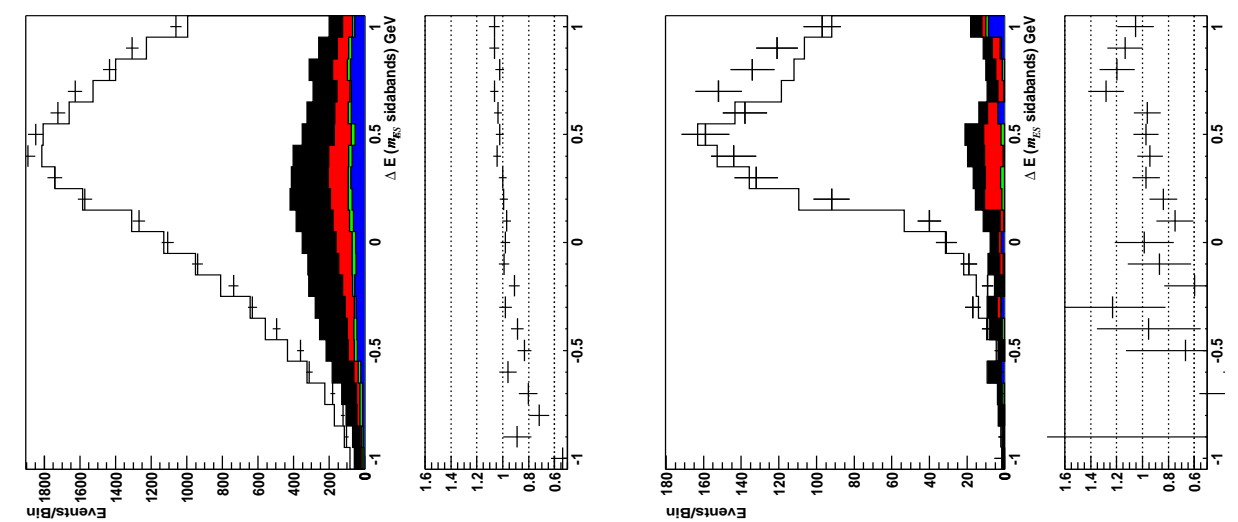

軑
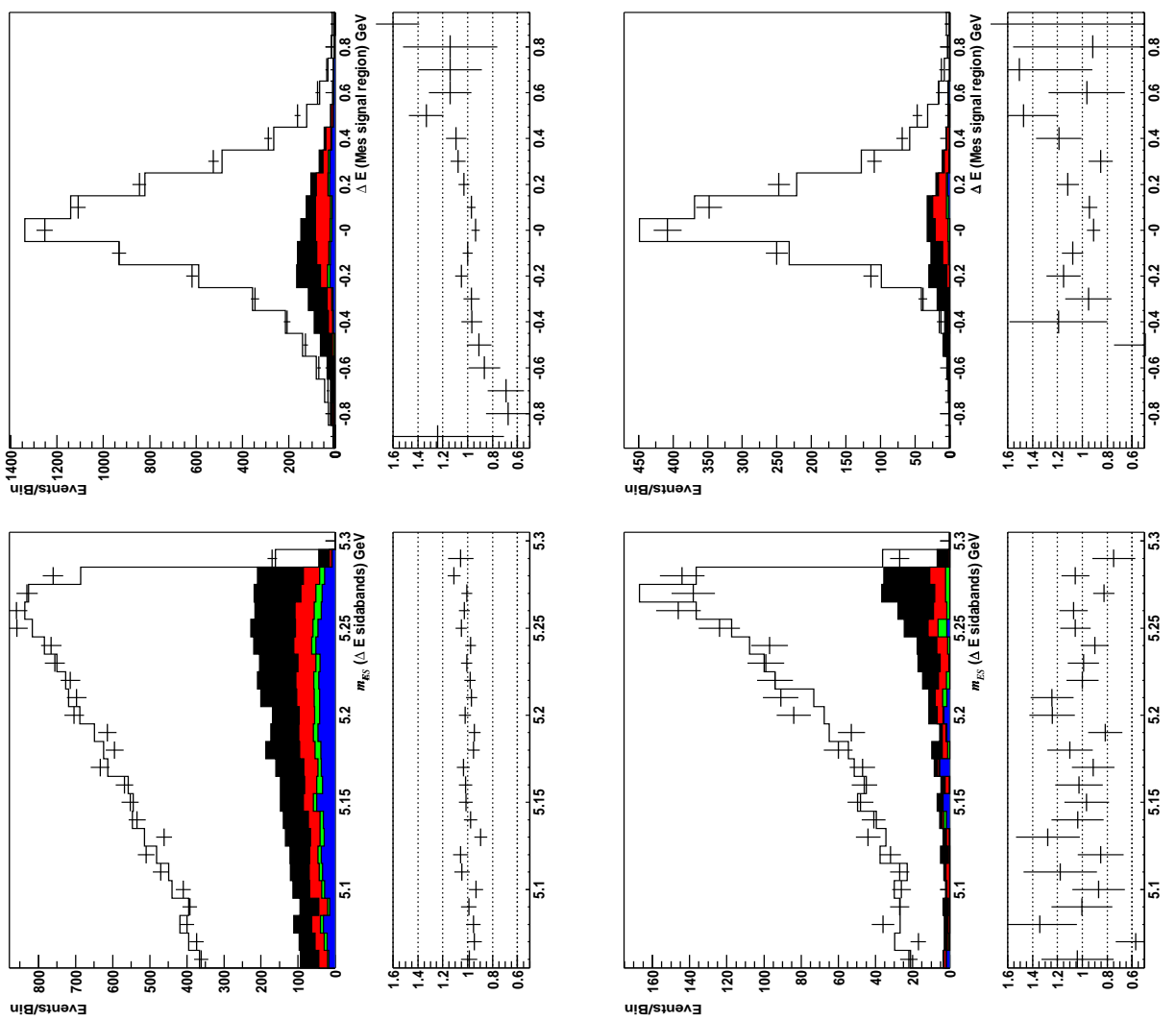

..$\Xi$

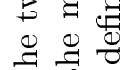

嵌

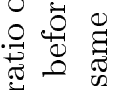

赵

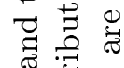

هీ

范客言

要

$\mp$ 담

$+\tilde{D}_{0} 0$

品 虽

ฮี

氜蛅

चี

.

䨔它

का

궈요

نี

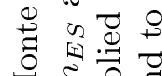

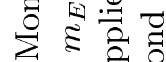

चै के की

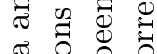

䨔.

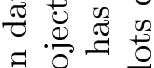

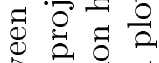

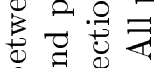

范

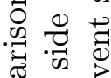

究的

융 휴
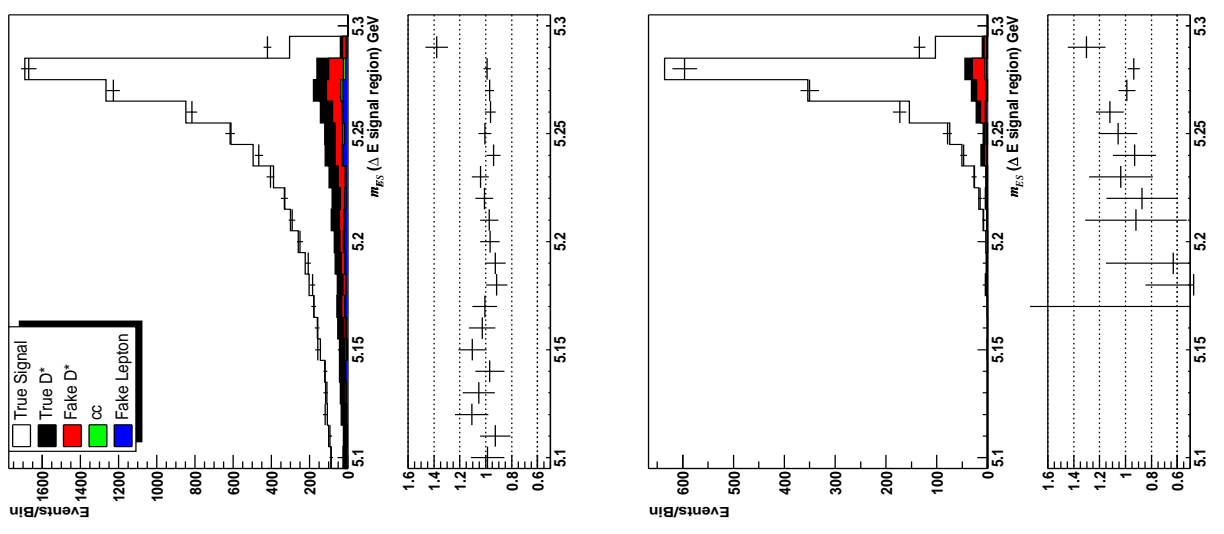

过

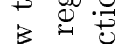

焉

융 栬

点它

틈

苛岂

苟葛

点. 
Table 4.5: Comparison of the number of events and the resulting efficiencies for the electron mode of the $\overline{D^{0}} \rightarrow K^{+} \pi^{-}$control sample for data and MC events. The efficiencies are evaluated sequentially, i.e. with previous cuts applied. The numbers are determined by counting the number of events in the $\Delta E, m_{E S}$ fit plane.

\begin{tabular}{|l|r|r|c|}
\hline $\begin{array}{l}\text { Event Sample } \\
\text { Criterion }\end{array}$ & $\begin{array}{r}\text { MC } \\
\text { eff }\end{array}$ & $\begin{array}{r}\text { Data } \\
\text { eff }\end{array}$ & Data/MC \\
\hline Events after $D^{*}$ selection & $17340 \pm 120$ & $16930 \pm 130$ & \\
\hline$R_{2}<0.4$ & $0.946 \pm 0.001$ & $0.953 \pm 0.002$ & $1.007 \pm 0.002$ \\
$L_{2}$ vs cos $\Delta \theta_{\text {Thrust }}$ & $0.787 \pm 0.002$ & $0.783 \pm 0.003$ & $0.995 \pm 0.005$ \\
$\theta_{\text {miss }}$ vs $p_{\text {miss }}$ & $0.838 \pm 0.002$ & $0.841 \pm 0.003$ & $1.003 \pm 0.005$ \\
Total charge $|Q|<2$ & $0.865 \pm 0.002$ & $0.865 \pm 0.003$ & $1.001 \pm 0.005$ \\
$\left|\cos \theta_{B Y}\right|<1.1$ & $0.939 \pm 0.002$ & $0.943 \pm 0.002$ & $1.005 \pm 0.003$ \\
$\left|\Delta \theta_{\text {min }}\right|<0.6 \mathrm{rad}$ & $0.628 \pm 0.004$ & $0.632 \pm 0.005$ & $1.007 \pm 0.010$ \\
$\left|M_{\text {miss }}^{2} / 2 E_{\text {miss }}\right|<0.4 \mathrm{GeV}$ & $0.316 \pm 0.005$ & $0.337 \pm 0.006$ & $1.068 \pm 0.026$ \\
All cuts & $0.101 \pm 0.002$ & $0.109 \pm 0.002$ & $1.085 \pm 0.030$ \\
\hline
\end{tabular}

\subsection{Efficiencies}

The absolute efficiencies for the five signal modes, determined from Monte Carlo, are listed in Table 4.11. The table also shows the crossfeed efficiencies between modes, when decays from one signal type are found in another reconstruction mode.

Tables 4.12 to 4.16 show the efficiencies of individual cuts for the five reconstruction modes. The tables give the efficiencies for signal and the main sources of background. 
Table 4.6: Comparison of the number of events and the resulting efficiencies for the muon mode of the $\overline{D^{0}} \rightarrow K^{+} \pi^{-}$control sample for data and MC events. The efficiencies are evaluated sequentially, i.e. with previous cuts applied. The numbers are determined by counting the number of events in the $\Delta E, m_{E S}$ fit plane.

\begin{tabular}{|l|r|r|r|}
\hline $\begin{array}{l}\text { Event Sample } \\
\text { Criterion }\end{array}$ & $\begin{array}{r}\text { MC } \\
\text { eff }\end{array}$ & $\begin{array}{r}\text { Data } \\
\text { eff }\end{array}$ & Data/MC \\
\hline Events after $D^{*}$ selection & $15210 \pm 110$ & $14500 \pm 120$ & \\
\hline$R_{2}<0.4$ & $0.948 \pm 0.001$ & $0.950 \pm 0.002$ & $1.002 \pm 0.002$ \\
$L_{2}$ vs cos $\Delta \theta_{\text {Thrust }}$ & $0.783 \pm 0.003$ & $0.785 \pm 0.004$ & $1.002 \pm 0.006$ \\
$\theta_{\text {miss }}$ vs $p_{\text {miss }}$ & $0.847 \pm 0.003$ & $0.847 \pm 0.003$ & $1.000 \pm 0.005$ \\
Total charge $|Q|<2$ & $0.868 \pm 0.003$ & $0.863 \pm 0.004$ & $0.995 \pm 0.005$ \\
$\left|\cos \theta_{B Y}\right|<1.1$ & $0.952 \pm 0.002$ & $0.956 \pm 0.002$ & $1.004 \pm 0.003$ \\
$\left|\Delta \theta_{\text {min }}\right|<0.6 \mathrm{rad}$ & $0.614 \pm 0.004$ & $0.620 \pm 0.006$ & $1.010 \pm 0.011$ \\
$\left|M_{\text {miss }}^{2} / 2 E_{\text {miss }}\right|<0.4 \mathrm{GeV}$ & $0.318 \pm 0.005$ & $0.339 \pm 0.007$ & $1.066 \pm 0.027$ \\
All cuts & $0.101 \pm 0.002$ & $0.110 \pm 0.003$ & $1.080 \pm 0.032$ \\
\hline
\end{tabular}

Table 4.7: Comparison of the number of events and the resulting efficiencies for the electron mode of the $\overline{D^{0}} \rightarrow K^{+} \pi^{-} \pi^{0}$ control sample for data and MC. The efficiencies are evaluated sequentially, i.e. with previous cuts applied. The numbers are determined by counting the number of events in the $\Delta E, m_{E S}$ fit plane.

\begin{tabular}{|l|r|r|r|}
\hline $\begin{array}{l}\text { Event Sample } \\
\text { Criterion }\end{array}$ & $\begin{array}{r}\text { MC } \\
\text { eff }\end{array}$ & $\begin{array}{r}\text { Data } \\
\text { eff }\end{array}$ & Data/MC \\
\hline Events after $D^{*}$ selection & $31850 \pm 160$ & $32060 \pm 180$ & \\
\hline$R_{2}<0.4$ & $0.958 \pm 0.001$ & $0.959 \pm 0.001$ & $1.001 \pm 0.001$ \\
$L_{2}$ vs cos $\Delta \theta_{\text {Thrust }}$ & $0.764 \pm 0.002$ & $0.761 \pm 0.002$ & $0.996 \pm 0.004$ \\
$\theta_{\text {miss }}$ vs $p_{\text {miss }}$ & $0.832 \pm 0.002$ & $0.838 \pm 0.002$ & $1.008 \pm 0.004$ \\
Total charge $|Q|<2$ & $0.852 \pm 0.002$ & $0.852 \pm 0.003$ & $1.000 \pm 0.004$ \\
$\left|\cos \theta_{B Y}\right|<1.1$ & $0.904 \pm 0.002$ & $0.906 \pm 0.002$ & $1.001 \pm 0.003$ \\
$\left|\Delta \theta_{\text {min }}\right|<0.6 \mathrm{rad}$ & $0.611 \pm 0.003$ & $0.619 \pm 0.004$ & $1.014 \pm 0.008$ \\
$\left|M_{\text {miss }}^{2} / 2 E_{\text {miss }}\right|<0.4 \mathrm{GeV}$ & $0.293 \pm 0.004$ & $0.304 \pm 0.005$ & $1.039 \pm 0.021$ \\
All cuts & $0.084 \pm 0.001$ & $0.089 \pm 0.002$ & $1.060 \pm 0.024$ \\
\hline
\end{tabular}


Table 4.8: Comparison of the number of events and the resulting efficiencies for the muon mode of the $\overline{D^{0}} \rightarrow K^{+} \pi^{-} \pi^{0}$ control sample for data and MC. The efficiencies are evaluated sequentially, i.e. with previous cuts applied. The numbers are determined by counting the number of events in the $\Delta E, m_{E S}$ fit plane.

\begin{tabular}{|l|r|r|r|}
\hline $\begin{array}{l}\text { Event Sample } \\
\text { Criterion }\end{array}$ & $\begin{array}{r}\text { MC } \\
\text { eff }\end{array}$ & $\begin{array}{r}\text { Data } \\
\text { eff }\end{array}$ & Data/MC \\
\hline Events after $D^{*}$ selection & $27600 \pm 140$ & $26840 \pm 160$ & \\
\hline$R_{2}<0.4$ & $0.954 \pm 0.001$ & $0.954 \pm 0.001$ & $1.000 \pm 0.002$ \\
$L_{2}$ vs cos $\Delta \theta_{\text {Thrust }}$ & $0.761 \pm 0.002$ & $0.763 \pm 0.003$ & $1.002 \pm 0.004$ \\
$\theta_{\text {miss }}$ vs $p_{\text {miss }}$ & $0.837 \pm 0.002$ & $0.842 \pm 0.003$ & $1.007 \pm 0.004$ \\
Total charge $|Q|<2$ & $0.857 \pm 0.002$ & $0.860 \pm 0.003$ & $1.003 \pm 0.004$ \\
$\left|\cos \theta_{B Y}\right|<1.1$ & $0.910 \pm 0.002$ & $0.915 \pm 0.002$ & $1.005 \pm 0.003$ \\
$\left|\Delta \theta_{\text {min }}\right|<0.6 \mathrm{rad}$ & $0.592 \pm 0.003$ & $0.599 \pm 0.004$ & $1.012 \pm 0.009$ \\
$\left|M_{\text {miss }}^{2} / 2 E_{\text {miss }}\right|<0.4 \mathrm{GeV}$ & $0.302 \pm 0.004$ & $0.324 \pm 0.005$ & $1.075 \pm 0.022$ \\
All cuts & $0.085 \pm 0.001$ & $0.094 \pm 0.002$ & $1.107 \pm 0.027$ \\
\hline
\end{tabular}

Table 4.9: Comparison of the number of events and the resulting efficiencies for the electron mode of the $\overline{D^{0}} \rightarrow K^{+} \pi^{-} \pi^{+} \pi^{-}$control sample for data and MC. The efficiencies are evaluated sequentially, i.e. with previous cuts applied. The numbers are determined by counting the number of events in the $\Delta E, m_{E S}$ fit plane.

\begin{tabular}{|l|r|r|c|}
\hline \hline $\begin{array}{l}\text { Event Sample } \\
\text { Criterion }\end{array}$ & $\begin{array}{r}\text { MC } \\
\text { eff }\end{array}$ & $\begin{array}{r}\text { Data } \\
\text { eff }\end{array}$ & Data/MC \\
\hline Events after $D^{*}$ selection & $14090 \pm 100$ & $13310 \pm 120$ & \\
\hline$R_{2}<0.4$ & $0.956 \pm 0.001$ & $0.963 \pm 0.002$ & $1.007 \pm 0.002$ \\
$L_{2}$ vs cos $\Delta \theta_{\text {Thrust }}$ & $0.759 \pm 0.003$ & $0.760 \pm 0.004$ & $1.000 \pm 0.006$ \\
$\theta_{\text {miss }}$ vs $p_{\text {miss }}$ & $0.830 \pm 0.003$ & $0.840 \pm 0.004$ & $1.012 \pm 0.006$ \\
Total charge $|Q|<2$ & $0.855 \pm 0.003$ & $0.858 \pm 0.004$ & $1.003 \pm 0.006$ \\
$\left|\cos \theta_{B Y}\right|<1.1$ & $0.913 \pm 0.002$ & $0.917 \pm 0.003$ & $1.005 \pm 0.005$ \\
$\left|\Delta \theta_{\text {min }}\right|<0.6 \mathrm{rad}$ & $0.608 \pm 0.005$ & $0.603 \pm 0.006$ & $0.992 \pm 0.012$ \\
$\left|M_{\text {miss }}^{2} / 2 E_{\text {miss }}\right|<0.4 \mathrm{GeV}$ & $0.293 \pm 0.005$ & $0.309 \pm 0.007$ & $1.056 \pm 0.032$ \\
All cuts & $0.084 \pm 0.002$ & $0.090 \pm 0.002$ & $1.077 \pm 0.037$ \\
\hline
\end{tabular}


Table 4.10: Comparison of the number of events and the resulting efficiencies for the muon mode of the $\overline{D^{0}} \rightarrow K^{+} \pi^{-} \pi^{+} \pi^{-}$control sample for data and MC. The efficiencies are evaluated sequentially, i.e. with previous cuts applied. The numbers are determined by counting the number of events in the $\Delta E, m_{E S}$ fit plane.

\begin{tabular}{|l|r|r|r|}
\hline $\begin{array}{l}\text { Event Sample } \\
\text { Criterion }\end{array}$ & $\begin{array}{r}\text { MC } \\
\text { eff }\end{array}$ & $\begin{array}{r}\text { Data } \\
\text { eff }\end{array}$ & Data/MC \\
\hline Events after $D^{*}$ selection & $12390 \pm 90$ & $11070 \pm 110$ & \\
\hline$R_{2}<0.4$ & $0.956 \pm 0.001$ & $0.961 \pm 0.002$ & $1.005 \pm 0.002$ \\
$L_{2}$ vs cos $\Delta \theta_{\text {Thrust }}$ & $0.762 \pm 0.003$ & $0.757 \pm 0.004$ & $0.994 \pm 0.007$ \\
$\theta_{\text {miss }}$ vs $p_{\text {miss }}$ & $0.837 \pm 0.003$ & $0.833 \pm 0.004$ & $0.995 \pm 0.006$ \\
Total charge $|Q|<2$ & $0.855 \pm 0.003$ & $0.855 \pm 0.004$ & $1.000 \pm 0.006$ \\
$\left|\cos \theta_{B Y}\right|<1.1$ & $0.917 \pm 0.003$ & $0.918 \pm 0.004$ & $1.001 \pm 0.005$ \\
$\left|\Delta \theta_{\text {min }}\right|<0.6 \mathrm{rad}$ & $0.590 \pm 0.005$ & $0.606 \pm 0.007$ & $1.027 \pm 0.014$ \\
$\left|M_{\text {miss }}^{2} / 2 E_{\text {miss }}\right|<0.4 \mathrm{GeV}$ & $0.299 \pm 0.006$ & $0.321 \pm 0.008$ & $1.072 \pm 0.035$ \\
All cuts & $0.085 \pm 0.002$ & $0.093 \pm 0.003$ & $1.094 \pm 0.041$ \\
\hline
\end{tabular}




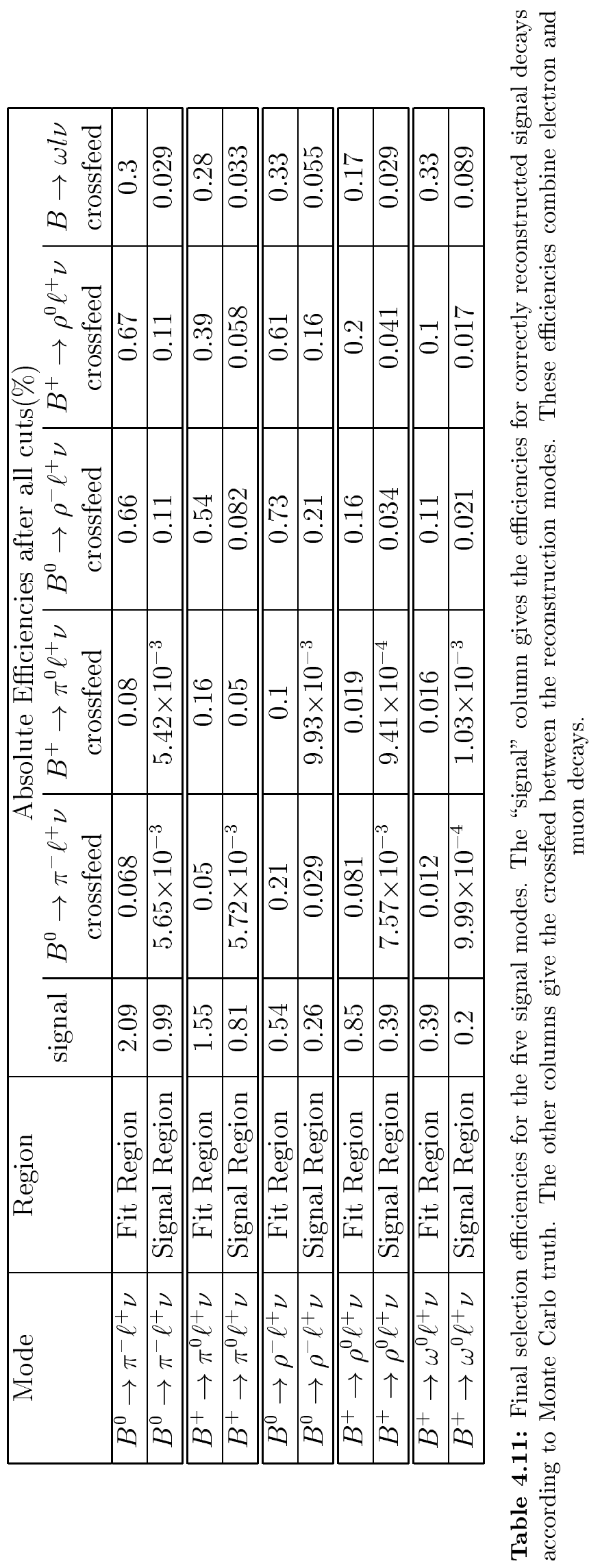




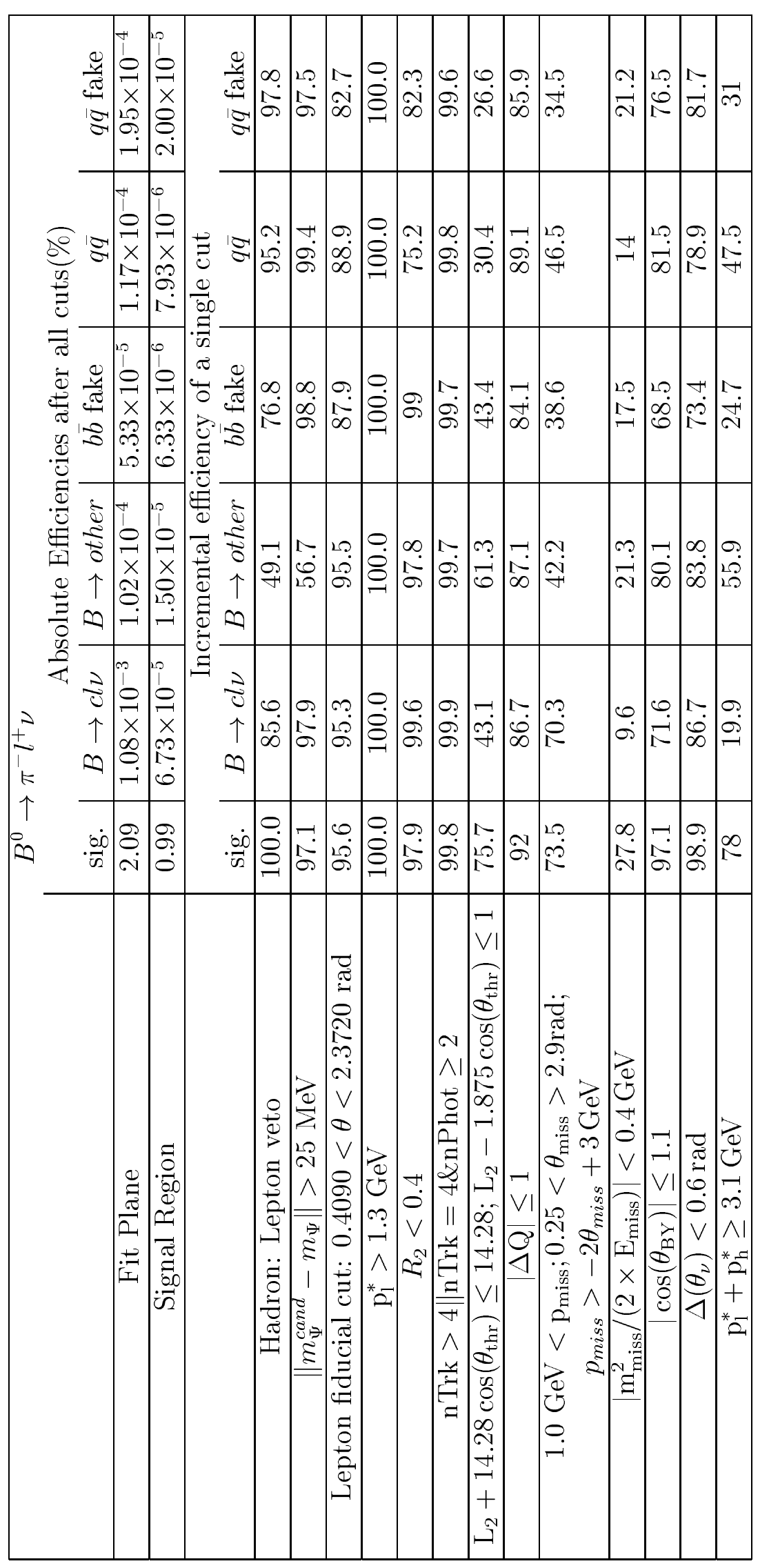

욤 号

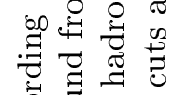

过

बक

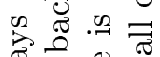

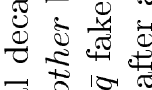

ฮี ซิ

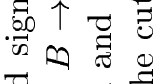

จ

声苛的

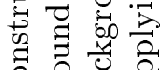

ชु क्षे

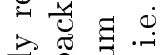

苞毛节

苞苛

包.

क ०ै 's

过的

링

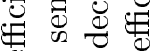

용 $\frac{\pi}{4}$

Ð

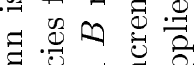

घี $. \Xi . \Xi$ శे

ชํํㄹ

500

낭

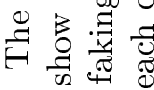

ชี चี

\&

$\pm \overrightarrow{0}$ व

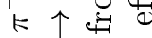

$\uparrow \curvearrowleft \Xi$

iิ

๑. 단

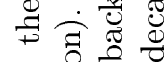

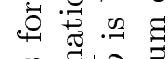

क 8 잉

过苛

जी की

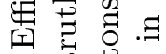

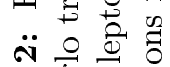

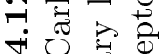

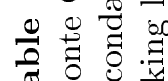

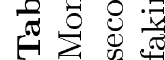




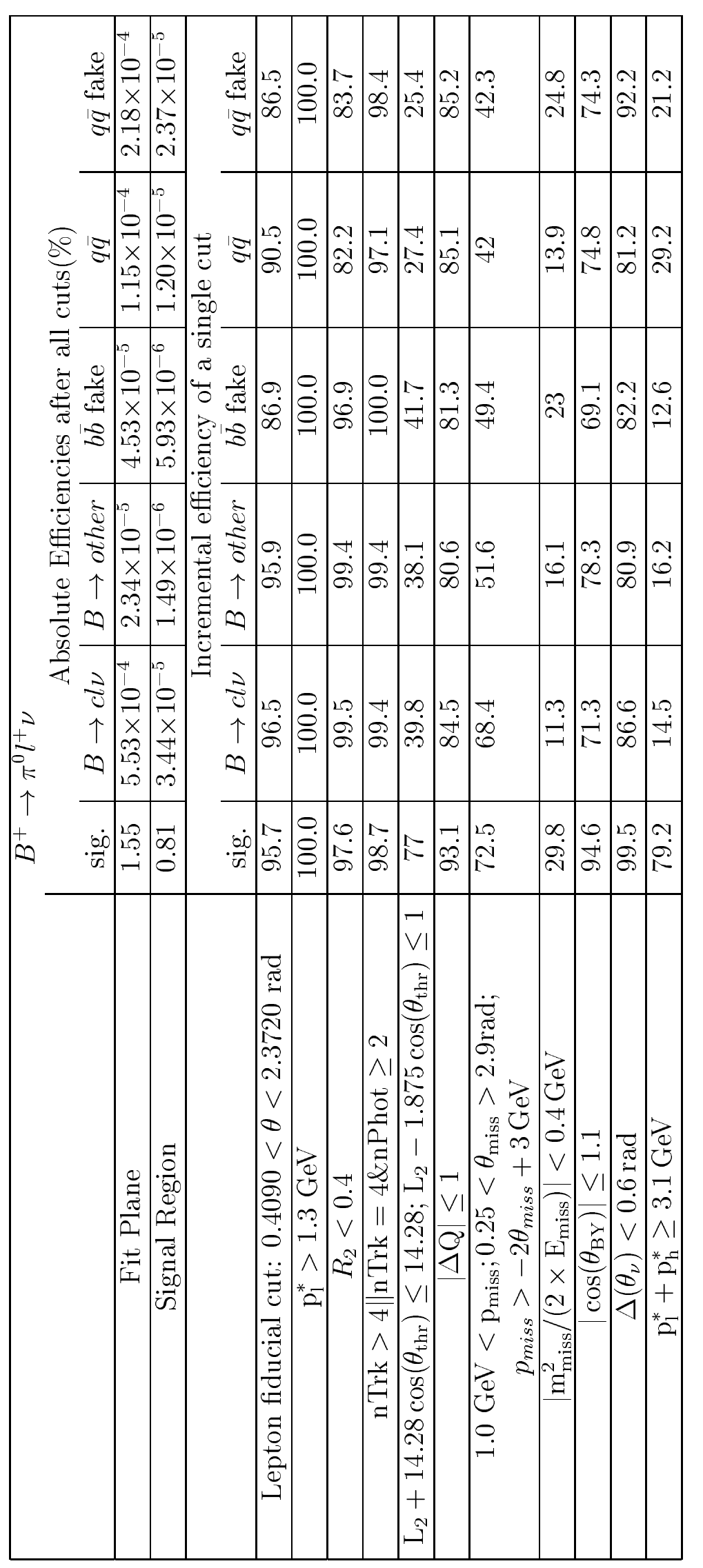

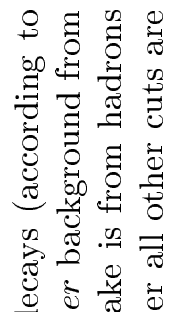
过娄 శ్ म

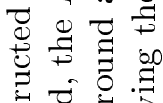

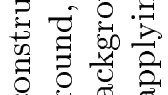
पूष $\rightarrow$ 它 苞寻吉 可

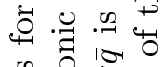
s 0

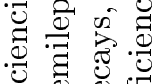
矛过 8 . † 寻.

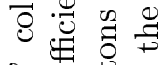
50 ज

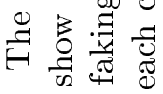

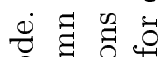

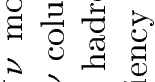
$\stackrel{2}{ \pm}$ जे

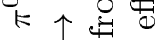
$\uparrow \oplus$ 焉

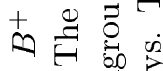

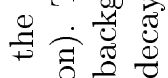

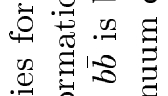

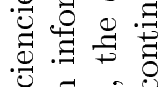
矛的㐘 玒焉

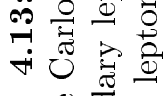

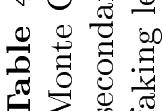




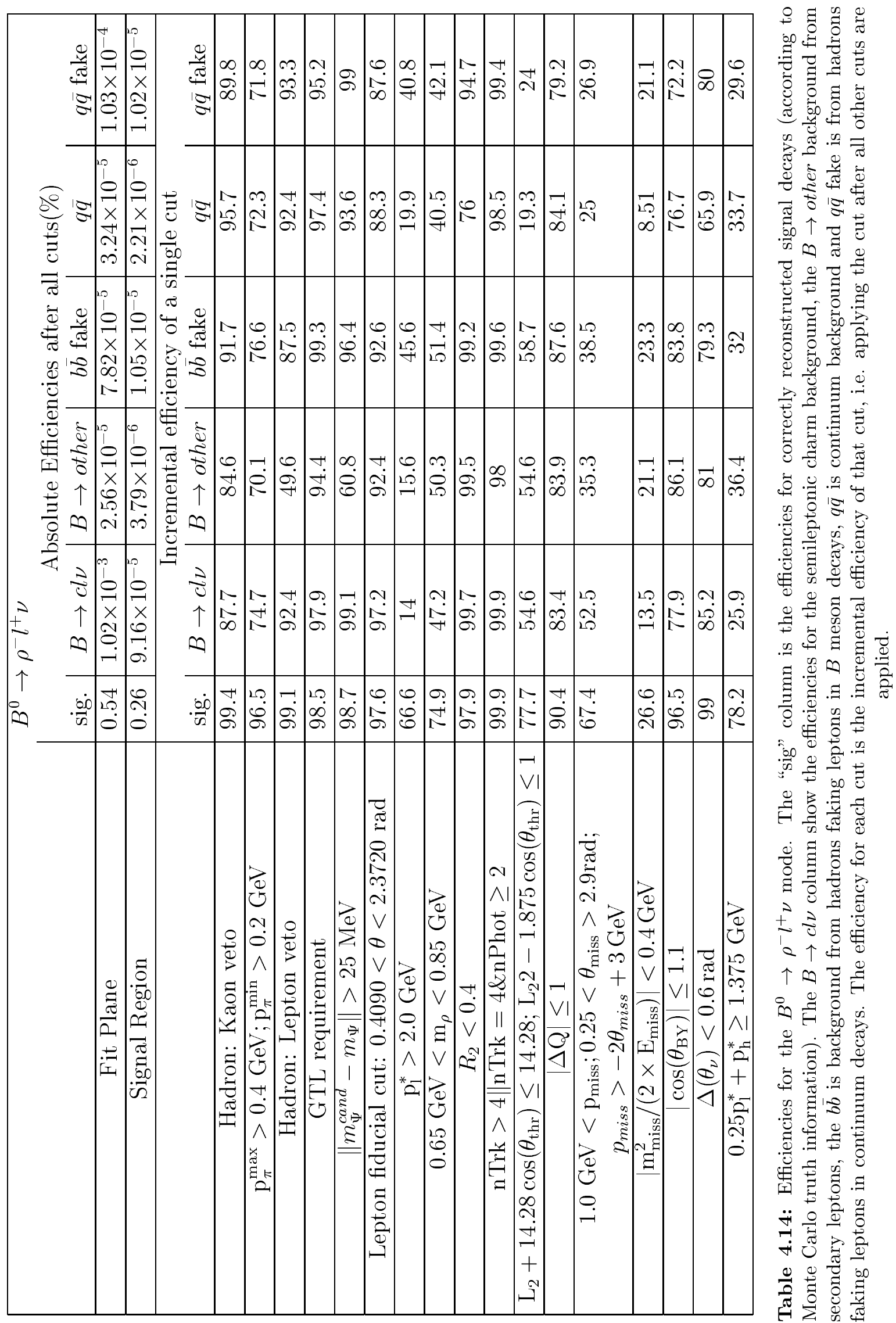




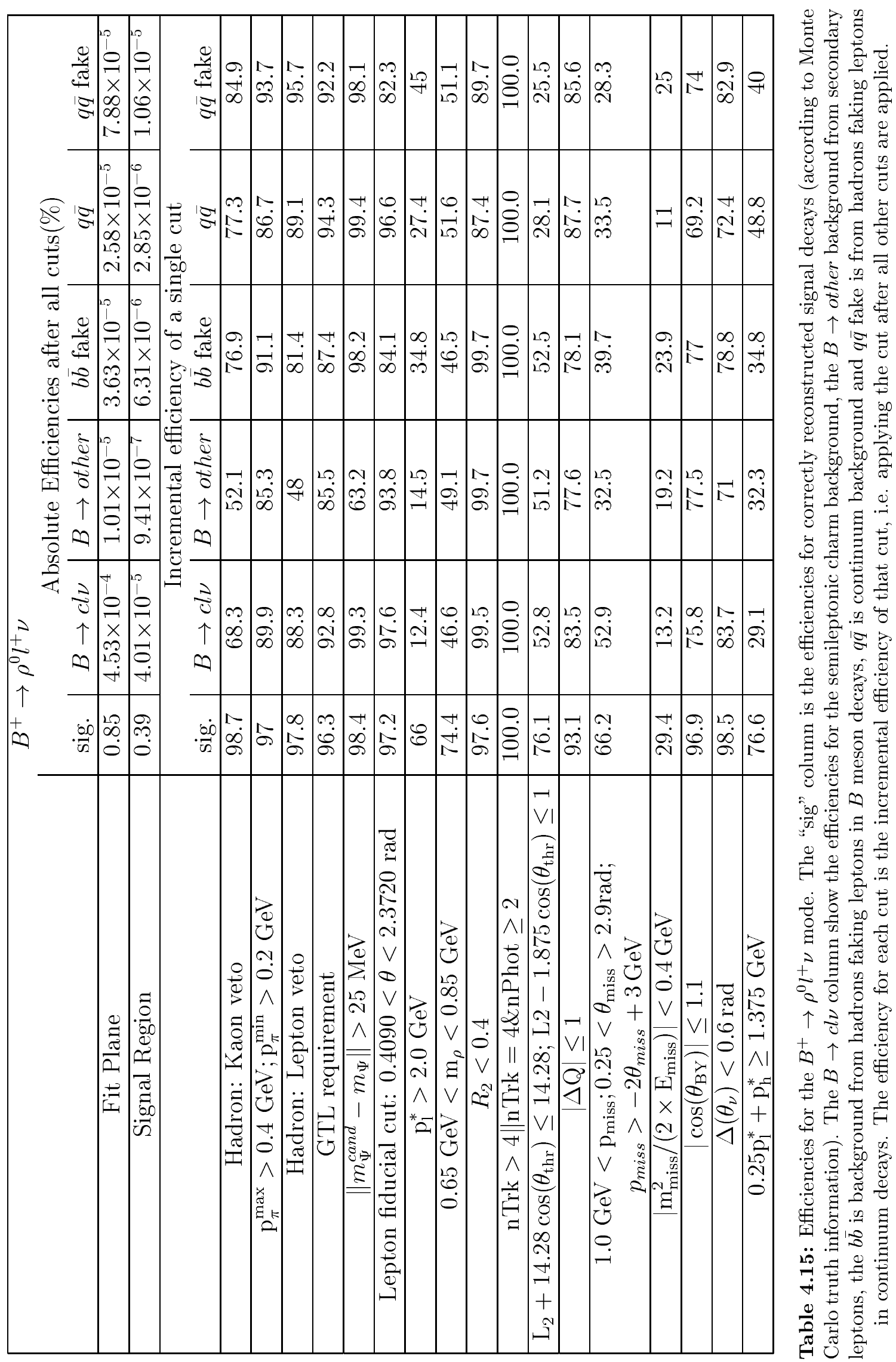




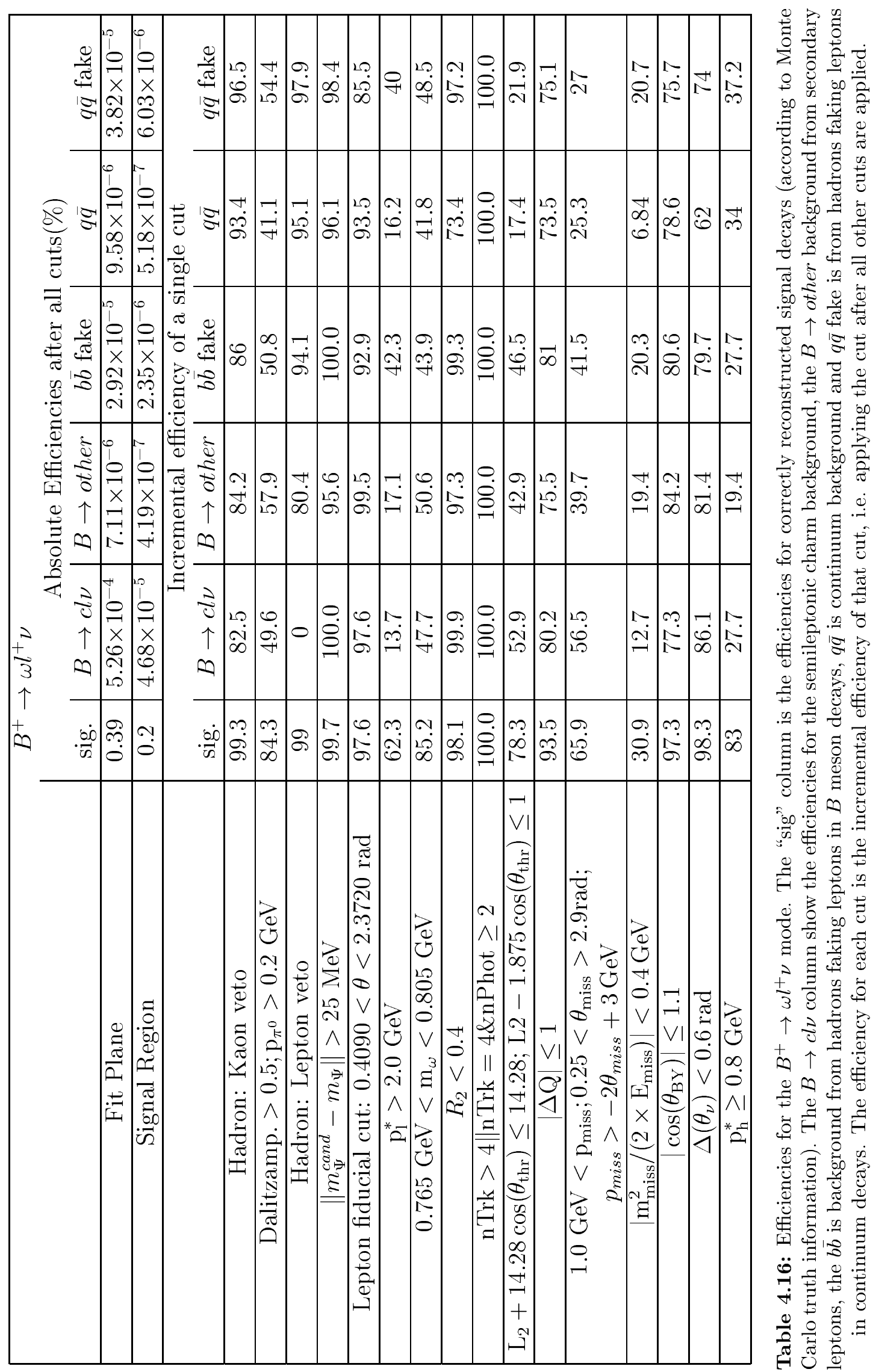




\section{Chapter 5}

\section{Maximum Likelihood Fit and Systematics}

\subsection{Introduction}

This chapter details the extraction of the charmless branching fractions $\mathcal{B}\left(B^{0} \rightarrow \pi^{-} l^{+} \nu\right)$, $\mathcal{B}\left(B^{0} \rightarrow \rho^{-} l^{+} \nu\right)$ and $\mathcal{B}\left(B \rightarrow \omega l^{+} \nu\right)$ from the selected samples. The fitting method and the breakdown of fit contributions is described. The branching fractions determined from the fit along with their statistical and systematic errors are given. Finally a value of $\left|V_{u b}\right|$ is extracted from the measured branching fractions.

\section{$5.2 \quad$ The Fit}

The branching fractions are extracted by fitting the data in the variables $\Delta E$ and $m_{E S}$. As the form of the distributions for the various fit components are difficult to parameterise analytically, a binned maximum likelihood fit is used. The fit is performed in the plane defined in Sec 4.10, i.e. $-0.95<\Delta E<0.95 \mathrm{GeV}, 5.095<$ $m_{E S}<5.305 \mathrm{GeV}$. 


\subsubsection{Fit Method}

The fit method used is described in [48]. It is in essence a binned maximum likelihood fit, using Monte Carlo events to determine the probability density functions (pdfs), that incorporates the statistical error of the Monte Carlo samples. The data are divided into $n$ bins with $d_{1}, d_{2} \ldots . . d_{n}$ data events in each bin. The predicted number of events in each bin $f_{i}\left(P_{1}, P_{2}, \ldots ., P_{m}\right)$, given in terms of the strengths $P_{j}$, from each source $j$ in bin $i$ is

$$
f_{i}=N_{D} \sum_{j=1}^{m} P_{j} w_{i j} a_{i j} / N_{j}
$$

where $a_{i j}$ is the number of Monte Carlo events from source $j$ in bin $i, w_{i j}$ are the weights assigned to the Monte Carlo, $N_{D}$ is the total number of events in the data sample and $N_{j}$ is the total in the Monte Carlo sample for source $j$;

$$
N_{D}=\sum_{i=1}^{n} d_{i}, \quad N_{j}=\sum_{i=1}^{n} w_{i j} a_{i j}
$$

Defining $p_{j}=N_{D} P_{j} / N_{j}$, equation 5.1 can be simplified to

$$
f_{i}=\sum_{j=1}^{m} p_{j} w_{i j} a_{i j}
$$

The probability of observing $d_{i}$ is described by a Poisson distribution with a mean $f_{i}$

$$
P\left(d_{i}\right)=e^{-f_{i}} \frac{f_{i}^{d_{i}}}{d_{i} !}
$$

so the $p_{j}$ are found by maximising the log likelihood

$$
\ln \mathcal{L}=\sum_{i=1}^{n} d_{i} \ln f_{i}-f_{i}
$$


This would be the case if $f_{i}$ did indeed correspond to the true mean of the Poisson distribution i.e. $a_{i j}$ were sufficiently large. What we actually want is

$$
f_{i}=\sum_{j=1}^{m} p_{j} w_{i j} A_{i j}
$$

where $A_{i j}$ is the unknown true expected number of events. The probability of getting the observed $a_{i j}$ is then

Incorporating this into $\ln \mathcal{L}$ gives

$$
P\left(a_{i j}\right)=e^{-A_{i j}} \frac{A_{i j}^{a_{i j}}}{a_{i j} !}
$$

$$
\ln \mathcal{L}=\sum_{i=1}^{n} d_{i} \ln f_{i}-f_{i}+\sum_{i=1}^{n} \sum_{j=1}^{m} a_{i j} \ln A_{i j}-A_{i j}
$$

The maximum of the likelihood is found by differentiating with respect to $A_{i j}$ and setting the result equal to zero,

$$
\frac{d_{i} w_{i j} p_{j}}{f_{i}}-w_{i j} p_{j}+\frac{a_{i j}}{A_{i j}}-1=0 \quad \forall_{j, i}
$$

This can be written as

$$
1-\frac{d_{i}}{f_{i}}=\frac{1}{w_{i j} p_{i}}\left(\frac{a_{i j}}{A_{i j}}-1\right)
$$

since the left hand side depends only on $i$ we define

$$
t_{i}=1-\frac{d_{i}}{f_{i}}
$$

and

$$
A_{i j}=\frac{a_{i j}}{1+p_{j} w_{i j} t_{i}}
$$

If $d_{i}=0, t_{i}=1$ otherwise

$$
\frac{d_{i}}{1-t_{i}}=f_{i}=\sum_{j} p_{j} w_{i j} A_{i j}=\sum_{j} \frac{p_{j} w_{i j} a_{i j}}{1+p_{j} w_{i j} t_{i}}
$$

The fit then proceeds by maximising the likelihood given by equation 5.8 using the Minuit package [49] to find the values of $p_{j}$. At each stage in the maximisation process the values $A_{i j}$ are found by solving for $t_{i}$ in the $n$ equations 5.13 using the Newtonian method and then using equation 5.12. 


\subsubsection{Fit Contributions}

The fit is performed for the five reconstructed modes simultaneously. By examining the origin of the lepton in the candidate combination the Monte Carlo simulation is divided into several categories. The contributions to the fit are:-

- The signal contribution. The shapes for the five signal contributions are taken from the Monte Carlo simulation. Each mode is further divided into true signal and combinatorics, using the truth matching in the Monte Carlo simulation. The true signal contribution is required to have all its constituent particles correctly identified. It is possible that events from one of the five signal modes may contribute to another reconstruction mode. When this occurs we have a "crossfeed" contribution. Using isospin relations the number of fit parameters for the signal modes can be reduced from five to three, or even two,

$$
\begin{aligned}
\Gamma\left(B^{0} \rightarrow \pi^{-} l^{+} \nu\right) & =2 \Gamma\left(B^{+} \rightarrow \pi^{0} l^{+} \nu\right) \\
\Gamma\left(B^{0} \rightarrow \rho^{-} l^{+} \nu\right) & =2 \Gamma\left(B^{+} \rightarrow \rho^{0} l^{+} \nu\right) \\
& \simeq 2 \Gamma\left(B^{+} \rightarrow \omega l^{+} \nu\right) .
\end{aligned}
$$

In this analysis the isospin relations 5.14 and 5.15 are used to constrain the branching fractions of the $\pi$ and $\rho$ modes. The branching fraction of the $\omega$ mode is an independent fit parameter.

- $b \rightarrow u l \nu$ downfeed. This is background originating from higher resonances of charmless mesons, or non-resonant decays to final states involving only pions. The shape of this contribution is determined from Monte Carlo events. The normalisation of this background is fixed to the most recent BABAR measurement of the inclusive charmless semileptonic rate $(2.24 \pm 0.27 \pm 0.26 \pm 0.39) \times$ $10^{-3}$ [50]. A systematic error is assigned to account for uncertainties in the composition and normalisation of this background.

- $b \rightarrow c l \nu$ decays. This background is due to charmed semileptonic decays. The shape of this background is determined from the Monte Carlo simulation. The normalisation is allowed to float in the fit. 
- Background from secondary leptons. This background includes decays such as $B \rightarrow J / \psi X, J / \psi \rightarrow l^{+} l^{-}$. This background represents only a small contribution to the total number of events. It is combined with the $b \rightarrow c l \nu$ background in the fit.

- Fake lepton background. This category consists of candidates where a hadron has been incorrectly identified as the lepton. The shape and normalisation of this background has been determined using the method outlined in Sec. 4.6.3.

- Non- $B B$ background. This includes all backgrounds originating from non- $B$ events, including $q \bar{q}$ and leptonic events. Again the shapes for this category of background are taken from Monte Carlo events. This background estimation suffers from very limited statistics. The normalisation of this background is determined by comparing the Monte Carlo simulation to the off-resonance data after backing off the cuts on the event shape variables $R_{2}$ and the combination of $L_{2}$ and $\cos \theta_{\text {Thrust }}$.

These contributions lead to a total of 11 fit parameters which are shown in Table 5.1 and outlined below. Eight of the parameters are free to float in the fit, the remaining three are fixed. The parameters are defined as follows.

- The five signal branching fractions are combined using isospin relations to give three free fit parameters,

- five fit parameters are $b \rightarrow c l \nu$ scale factors relative to the normalisation expected from the Monte Carlo simulation; one for each reconstruction mode,

- a parameter is a scale factor for the $b \rightarrow u l \nu$ downfeed; this is fixed in the fit to the most recent BABAR measurement,

- a scale factor for the non- $B$ background; this is fixed in the fit,

- a scale factor for the fake lepton background; this is fixed in the fit. 
As an illustration consider the $B \rightarrow \pi^{-} l^{+} \nu$ mode. The function to be determined is

$$
f_{i}=\sum_{j=1}^{m} p_{j} w_{i j} a_{i j}
$$

where the coefficients $p_{j}$ are given by,

$$
\begin{aligned}
& p_{1}=\eta_{1} \cdot 2 f_{00} \cdot N_{B \bar{B}} \cdot \epsilon_{\pi^{+}}^{\pi^{+}} \cdot 1 / a^{\pi^{+}}, \\
& p_{2}=\frac{1}{2} \eta_{1} \cdot 2\left(1-f_{00}\right) \cdot N_{B \bar{B}} \cdot \frac{\tau^{+}}{\tau^{0}} \cdot \epsilon_{\pi^{+}}^{\pi^{0}} \cdot 1 / a^{\pi^{0}}, \\
& p_{3}=\eta_{2} \cdot 2 f_{00} \cdot N_{B \bar{B}} \cdot \epsilon_{\pi^{+}}^{\rho^{+}} \cdot 1 / a^{\rho^{+}}, \\
& p_{4}=\frac{1}{2} \eta_{2} \cdot 2\left(1-f_{00}\right) \cdot N_{B \bar{B}} \cdot \frac{\tau^{+}}{\tau^{0}} \cdot \epsilon_{\pi^{+}}^{\rho^{0}} \cdot 1 / a^{\rho^{0}}, \\
& p_{5}=\eta_{3} \cdot 2\left(1-f_{00}\right) \cdot N_{B \bar{B}} \cdot \epsilon_{\pi^{+}}^{\omega} \cdot 1 / a^{\omega}, \\
& p_{6}=\eta_{4}, \\
& p_{7}=\eta_{9}, \\
& p_{8}=\eta_{10}=1.07, \\
& p_{9}=\eta_{11}=1,
\end{aligned}
$$

where $\epsilon_{x}^{y}$ is the efficiency for reconstructing the decay type $y$ in the reconstruction mode $x$; the values $a^{z}$ are the sum of the Monte Carlo contributions to $z$ over all the bins in the histograms, $a_{z}=\sum_{i} w_{i z} a_{i z} \cdot \tau^{+} / \tau^{0}$ is the ratio of the lifetime of the charge and neutral $B$; the value used, $\tau^{+} / \tau^{0}=1.083 \pm 0.017$, is taken from [7]. The neutral $B$ fraction of the total $B$ production rate at the $\Upsilon(4 S)$ resonance $\left(f_{00}\right)$ is assumed to be equal to 0.5 .

The scale of the non- $B$ background is determined by fitting the non- $B$ Monte Carlo events and the fake contribution measured from data to the off-resonance data. For this measurement the cuts on the event shape variables $R_{2}$ and the combination of $L_{2}$ and $\cos \theta_{\text {Thrust }}$ are relaxed. This results in a scale factor of $1.07 \pm 0.06$ relative to the amount predicted by the Monte Carlo simulation. This parameter is fixed to this value in the later fits. The projection in $\Delta E$ and $m_{E S}$ of the fit to the off-resonance data for the $B^{0} \rightarrow \pi^{-} l^{+} \nu$ mode is shown in Fig. 5.1. The projections are divided into electrons and muons. 
Table 5.1: The table shows the parameters used in the fit. The last coloumn shows which parameters are free in the fit and which are fixed.

\begin{tabular}{|l|l|l|}
\hline$\eta_{1}$ & $\mathcal{B}\left(B \rightarrow \pi^{ \pm} l \nu\right)$ & free \\
$\eta_{2}$ & $\mathcal{B}\left(B \rightarrow \rho^{ \pm} l \nu\right)$ & free \\
$\eta_{3}$ & $\mathcal{B}(B \rightarrow \omega l \nu)$ & free \\
$\eta_{4}$ & Scale factor for $B \rightarrow c l \nu+\pi$ decays & free \\
$\eta_{5}$ & Scale factor for $B \rightarrow c l \nu+\rho$ decays & free \\
$\eta_{6}$ & Scale factor for $B \rightarrow c l \nu+\omega$ decays & free \\
$\eta_{7}$ & Scale factor for $B \rightarrow c l \nu+\pi^{0}$ decays & free \\
$\eta_{8}$ & Scale factor for $B \rightarrow c l \nu+\rho^{0}$ decays & free \\
$\eta_{9}$ & Scale factor for $B \rightarrow u l \nu$ & fixed \\
$\eta_{10}$ & Scale factor for non- $B$ decays & fixed \\
$\eta_{11}$ & Scale factor for fake lepton decays & fixed \\
\hline
\end{tabular}
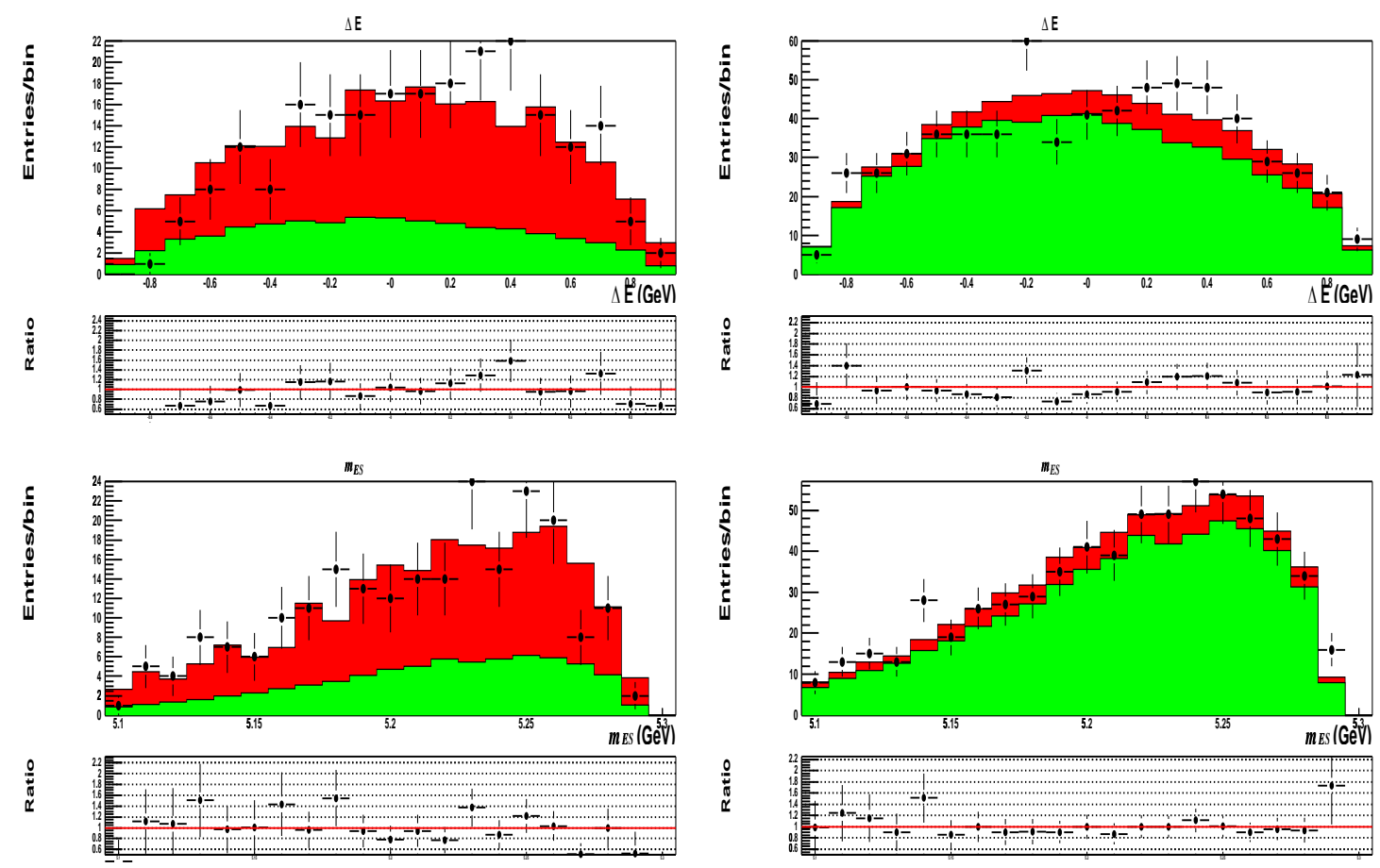

Figure 5.1: Projections of the fit to the off-resonanc data for the $B^{0} \rightarrow$ $\pi^{-} l^{+} \nu$ reconstruction mode. The lower plot in each set shows the ratio of the data and the fit projection. The green histogram is the fake lepton contribution and the red the non- $B$ background from Monte Carlo. The plots on the left are for electrons and the right muons. The fake lepton background dominates for the muon.

\subsubsection{Fit Binning}

The binning used in the fit is chosen in an effort to provide maximum sensitivity to the signal whilst being relatively insensitive to any differences between the shape of 
the data and Monte Carlo simulation. The binning chosen is shown in Fig. 5.2.

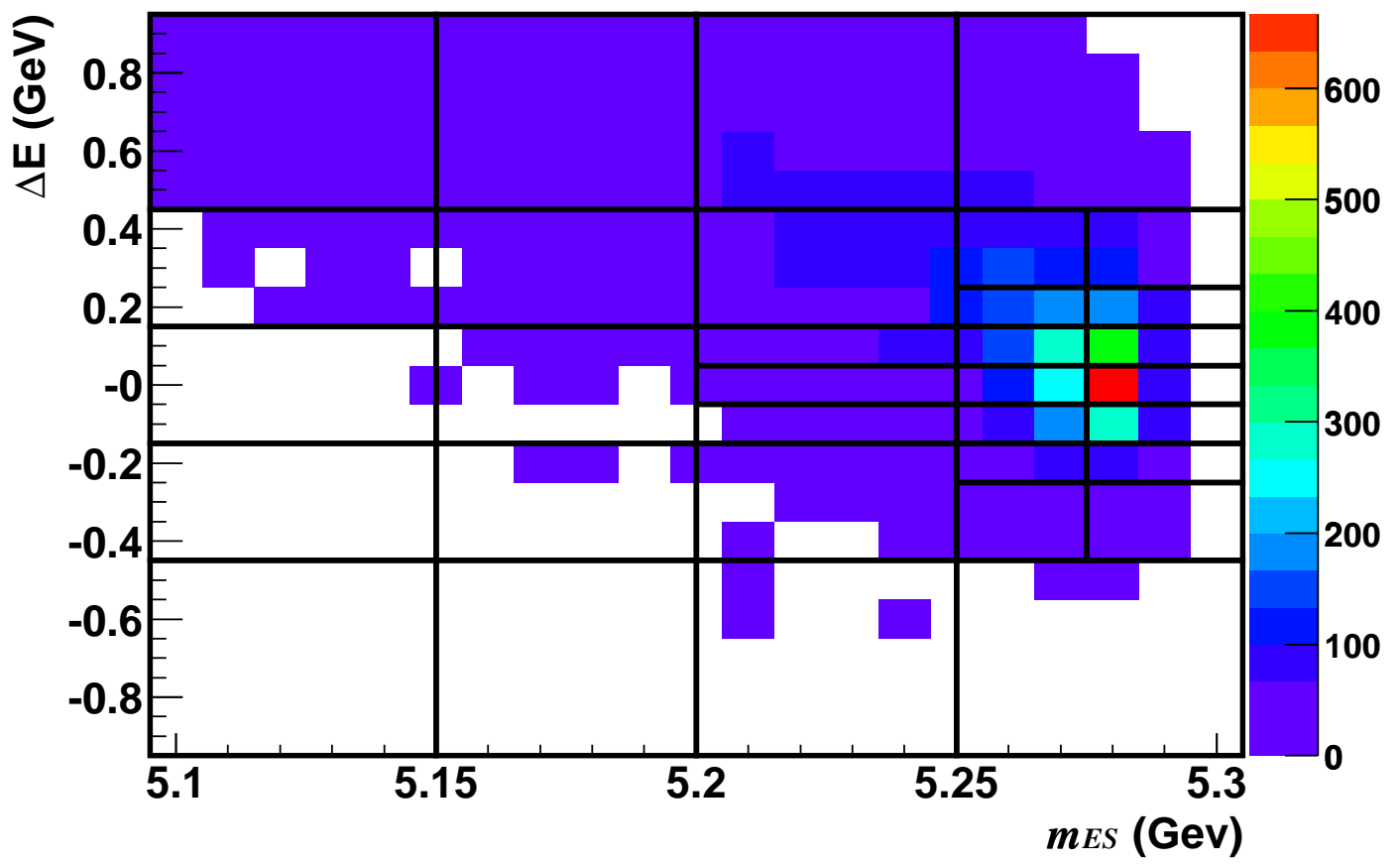

Figure 5.2: The distribution of the $B \rightarrow \pi^{-} l^{+} \nu$ signal events across the fit plane. The grid shows the choice of binning used in the fit.

\subsubsection{Fit Validation}

In order to validate the fitting procedure a number of toy Monte Carlo fits have been performed. As the amount of Monte Carlo simulation available is limited, it is not practical to follow the standard procedure of fitting many independent Monte Carlo samples. Instead the data is simulated by applying a Poisson fluctuation to the expected number of entries in each bin for each contribution, determined from Monte Carlo, and using this distribution as the new data set. The fit parameters used to generate the toy experiments are those determined from the final fit to data. This procedure relies on the number of events for each contribution $\left(N_{i}\right)$ being a good approximation for the true expected value. For bins with $N_{i}=0$, this is not the case and further will result in zero events for all toy samples. This could cause a bias in the result. In order to reduce the number of bins with zero entries the electron and muon events have been combined before generating the toy Monte 
Carlo samples. Fig. 5.3 shows the pull distributions from the 1000 toy experiments, where the pull is defined as

$$
\text { Pull }=\frac{\mathcal{B}_{\text {fitted }}-\mathcal{B}_{\text {generated }}}{\sigma_{\mathcal{B}_{\text {fitted }}}}
$$

The pulls determined are, $\operatorname{pull}\left(\mathcal{B}\left(B^{0} \rightarrow \pi^{-} l^{+} \nu\right)\right)=0.004$ with a width of 0.94 , $\operatorname{pull}\left(\mathcal{B}\left(B^{0} \rightarrow \rho^{-} l^{+} \nu\right)\right)=-0.13$ with a spread of 0.93 and $\operatorname{pull}\left(\mathcal{B}\left(B^{+} \rightarrow \omega l^{+} \nu\right)\right)=$ -0.05 with a spread of 0.99 .
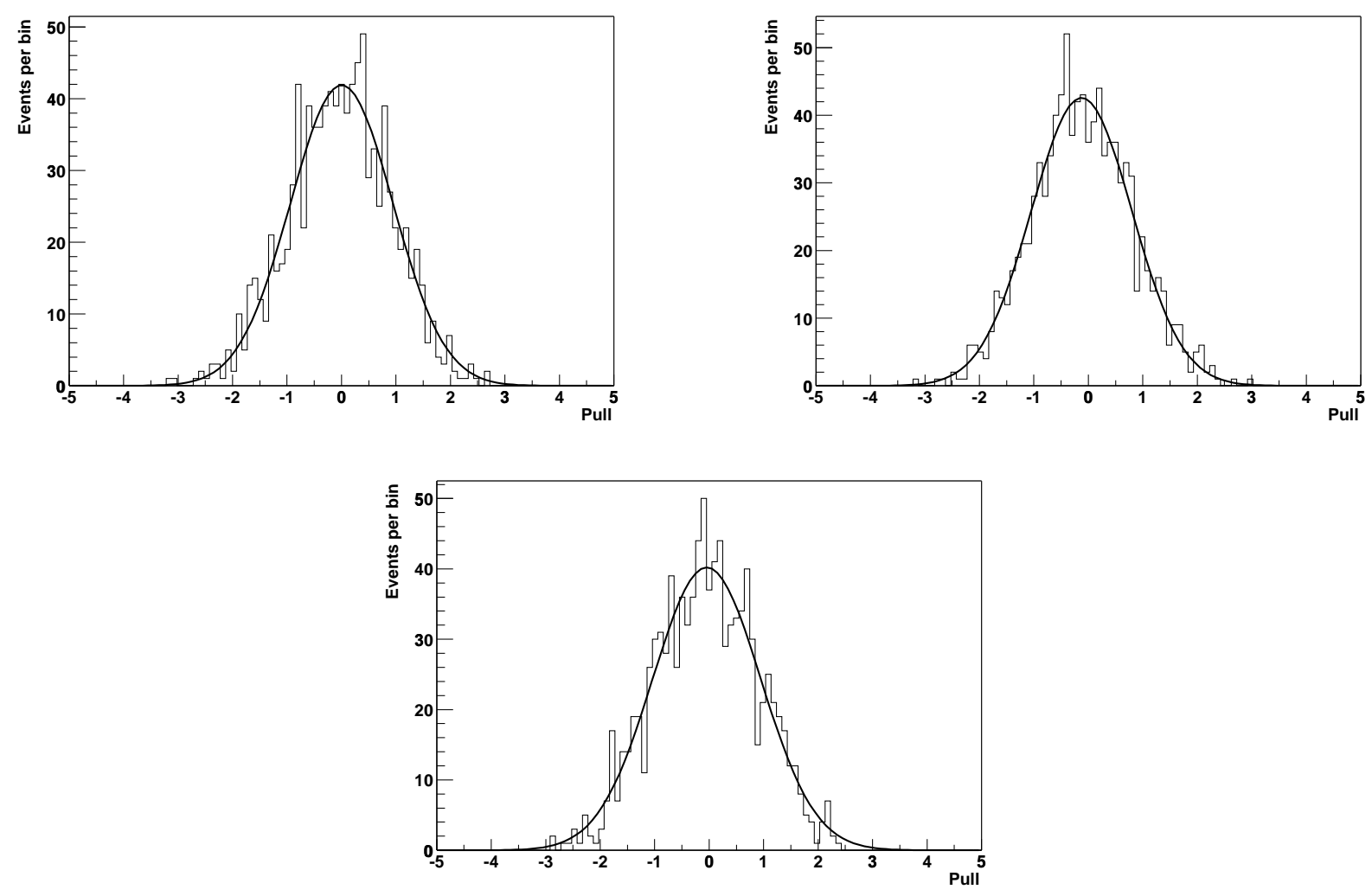

Figure 5.3: The pull distributions for a set of 1000 toy Monte Carlo experiments. The top left plot shows the pull for $\mathcal{B}\left(B \rightarrow \pi^{ \pm} l \nu\right)$, the top right the pull for $\mathcal{B}\left(B \rightarrow \rho^{ \pm} l \nu\right)$ and the bottom plot $\mathcal{B}(B \rightarrow \omega l \nu)$. The distributions are fitted with a Gaussian function 


\begin{tabular}{|c|c|}
\hline Fit Parameter & $\eta_{i} \pm \Delta \eta_{i}$ \\
\hline$\eta_{1}\left(\mathcal{B}\left(B \rightarrow \pi^{ \pm} \ell \nu\right)\right)$ & $(1.67 \pm 0.11) \times 10^{-4}$ \\
$\eta_{2}\left(\mathcal{B}\left(B \rightarrow \rho^{ \pm} \ell \nu\right)\right)$ & $(2.10 \pm 0.18) \times 10^{-4}$ \\
$\eta_{3}(\mathcal{B}(B \rightarrow \omega \ell \nu))$ & $(1.69 \pm 0.26) \times 10^{-4}$ \\
\hline$\eta_{4}(b \rightarrow c \ell \nu+\pi)$ & $1.12 \pm 0.04$ \\
$\eta_{5}(b \rightarrow c \ell \nu+\rho)$ & $1.22 \pm 0.05$ \\
$\eta_{6}(b \rightarrow c \ell \nu+\omega)$ & $1.13 \pm 0.06$ \\
$\eta_{7}\left(b \rightarrow c \ell \nu+\pi^{0}\right)$ & $0.93 \pm 0.06$ \\
$\eta_{8}\left(b \rightarrow c \ell \nu+\rho^{0}\right)$ & $1.47 \pm 0.07$ \\
\hline
\end{tabular}

Table 5.2: The branching fractions and scale factors determined by the fit.

\subsection{Results}

\subsubsection{Branching Fraction Fit Results}

The parameters determined by the fit are shown in Table 5.2 and correlation coefficients between the fit parameters returned by Minuit are given in Table 5.3. The result includes data from all years of running and both electrons and muons. The measured branching fractions are

- $\mathcal{B}\left(B^{0} \rightarrow \pi^{-} l^{+} \nu\right)=(1.67 \pm 0.11 \pm 0.07) \times 10^{-4}$

- $\mathcal{B}\left(B^{0} \rightarrow \rho^{-} l^{+} \nu\right)=\left(2.10 \pm 0.18_{-0.28}^{+0.23}\right) \times 10^{-4}$

- $\mathcal{B}\left(B^{0} \rightarrow \omega l^{+} \nu\right)=(1.69 \pm 0.26 \pm 0.24) \times 10^{-4}$

where the errors are statistical and systematic respectively. The evaluation of the systematic errors is described in Sec. 5.4.

\subsubsection{Fit Projections}

Figures 5.4-5.8 show the projections of the fit results onto the $\Delta E$ and $m_{E S}$ variables. The projections are divided into signal regions $(-0.25<\Delta E<0.15 \mathrm{GeV}$ or $\left.5.25<m_{E S}<5.305 \mathrm{GeV}\right)$ and sideband regions using the variable not being plotted. Reasonable agreement is seen between data and the fit projections. 


\begin{tabular}{|c|l|l|l|l|l|l|l|l|l|}
\hline Parameter & \multicolumn{9}{|c|}{ Correlation Coefficents } \\
\hline & global & $\eta_{1}$ & $\eta_{2}$ & $\eta_{3}$ & $\eta_{4}$ & $\eta_{5}$ & $\eta_{6}$ & $\eta_{7}$ & $\eta_{8}$ \\
\hline$\eta_{1}$ & 0.40996 & 1.000 & -0.259 & 0.003 & -0.188 & 0.078 & 0.014 & -0.094 & 0.063 \\
$\eta_{2}$ & 0.66132 & -0.259 & 1.000 & -0.248 & -0.243 & -0.413 & 0.024 & -0.192 & -0.277 \\
$\eta_{3}$ & 0.53359 & 0.003 & -0.248 & 1.000 & -0.080 & -0.064 & -0.385 & -0.128 & -0.049 \\
$\eta_{4}$ & 0.39067 & -0.188 & -0.243 & -0.080 & 1.000 & 0.136 & 0.052 & 0.116 & 0.090 \\
$\eta_{5}$ & 0.44954 & 0.078 & -0.413 & -0.064 & 0.136 & 1.000 & 0.058 & 0.117 & 0.136 \\
$\eta_{6}$ & 0.39232 & 0.014 & 0.024 & -0.385 & 0.052 & 0.058 & 1.000 & 0.067 & 0.041 \\
$\eta_{7}$ & 0.30857 & -0.094 & -0.192 & -0.128 & 0.116 & 0.117 & 0.067 & 1.000 & 0.078 \\
$\eta_{8}$ & 0.30338 & 0.063 & -0.277 & -0.049 & 0.090 & 0.136 & 0.041 & 0.078 & 1.000 \\
\hline
\end{tabular}

Table 5.3: The correlations between fit parameters. The parameters are defined as in the Table 5.1 .
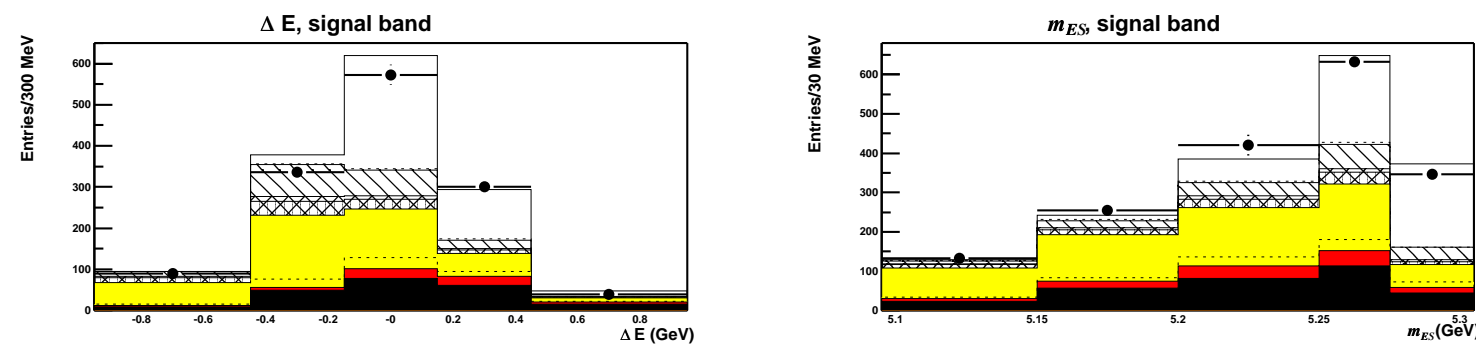

$\stackrel{\frac{0}{\bar{x}}}{x}$
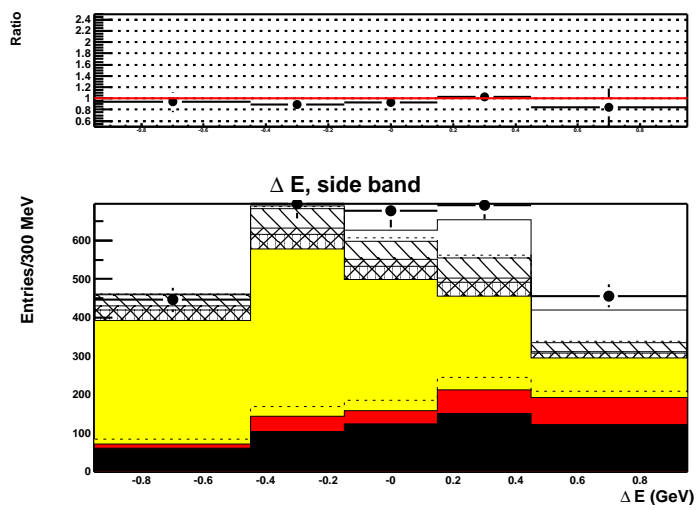

$\stackrel{\circ}{\frac{1}{x}}$

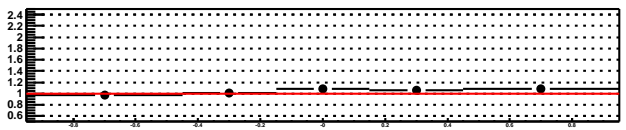

\section{$\frac{0}{\bar{x}}$}
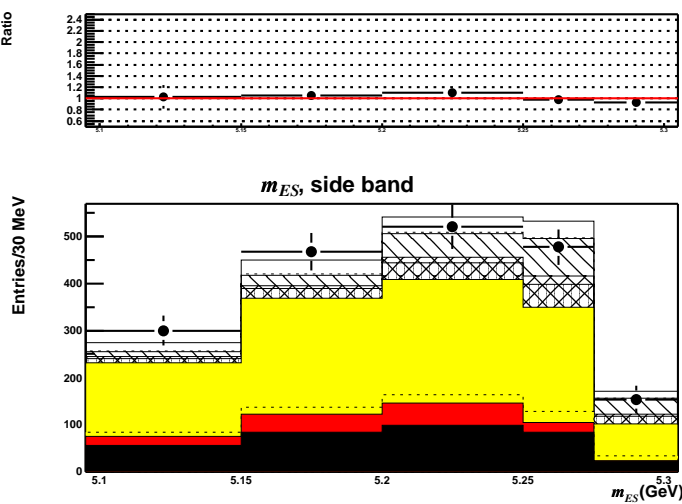

$\frac{\circ}{\frac{1}{x}}$

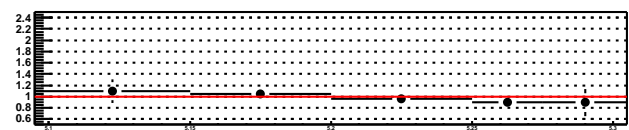

Figure 5.4: $\Delta E$ and $m_{E S}$ projections for $B^{0} \rightarrow \pi^{-} \ell^{+} \nu$. The left plots show the projections for $\Delta E$ in the $m_{E S}$ signal (top set of plots) and $m_{E S}$ sideband (bottom plots) regions. On the right are the corresponding plots for $m_{E S}$. The lower plot in each set shows the ratio of the data and the fit results in each bin. The histogram components, from bottom to top, are fake leptons (black), continuum (red), secondary leptons (yellow dashed), $b \rightarrow$ $c l \nu$ decays (yellow), $b \rightarrow u l \nu$ feeddown (vertical lines), vector mode crossfeed (cross hatched), pion cross-feed (open dashed histogram, this includes incorrectly reconstructed signal decays) and true signal decays (open). 

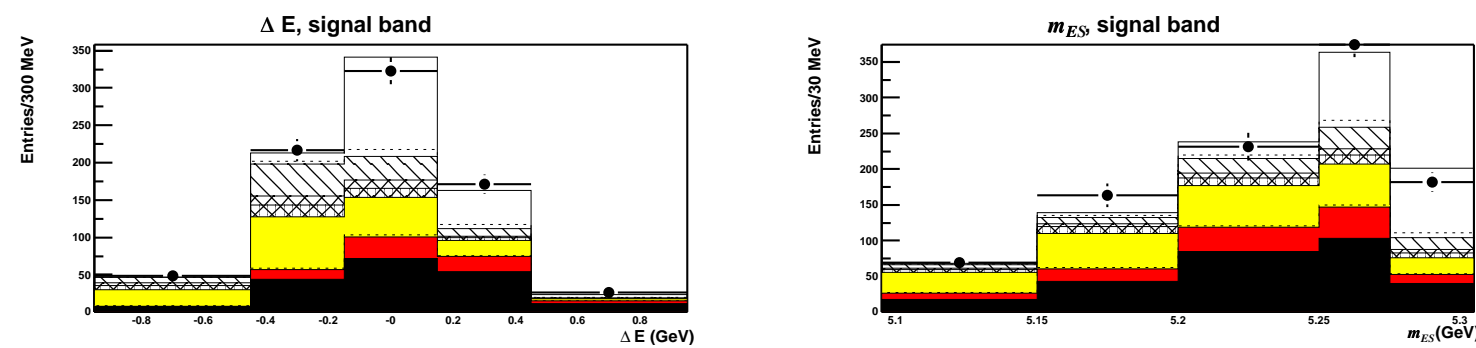

ㅇㅡㄸ

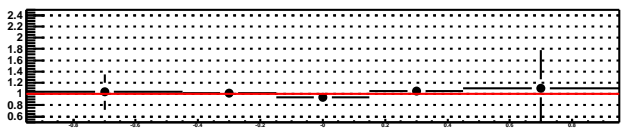

$\stackrel{\circ}{\frac{1}{\pi}}$
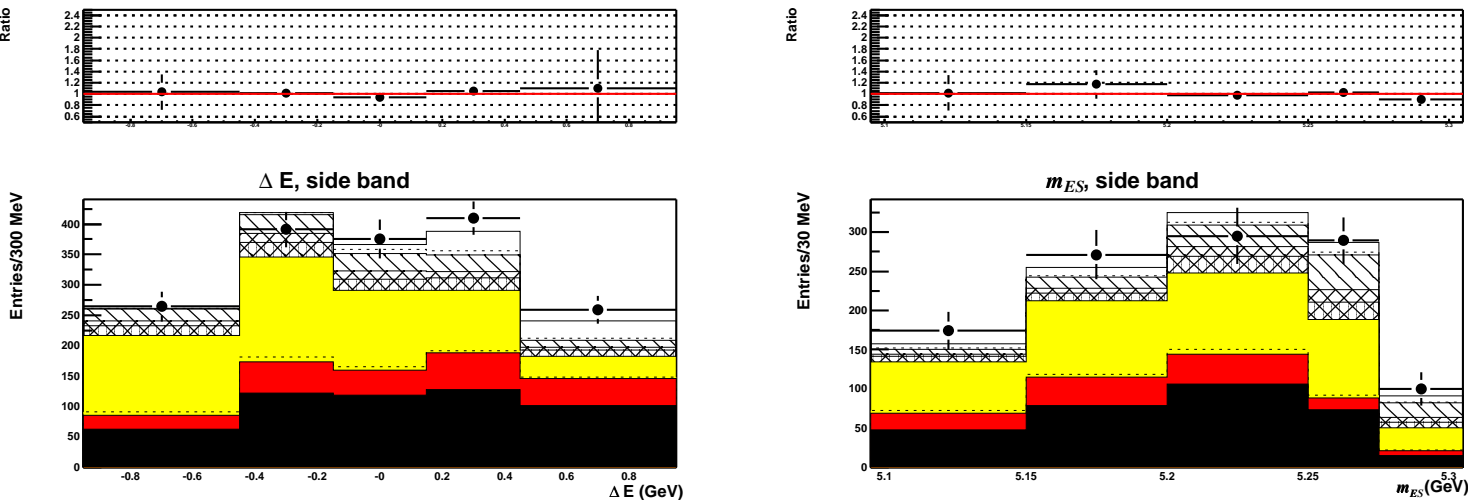

윯

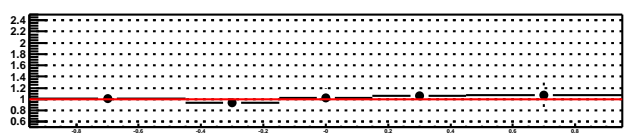

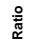

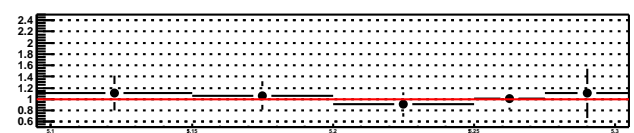

Figure 5.5: $\Delta E$ and $m_{E S}$ projections for $B^{+} \rightarrow \pi^{0} \ell^{+} \nu$.
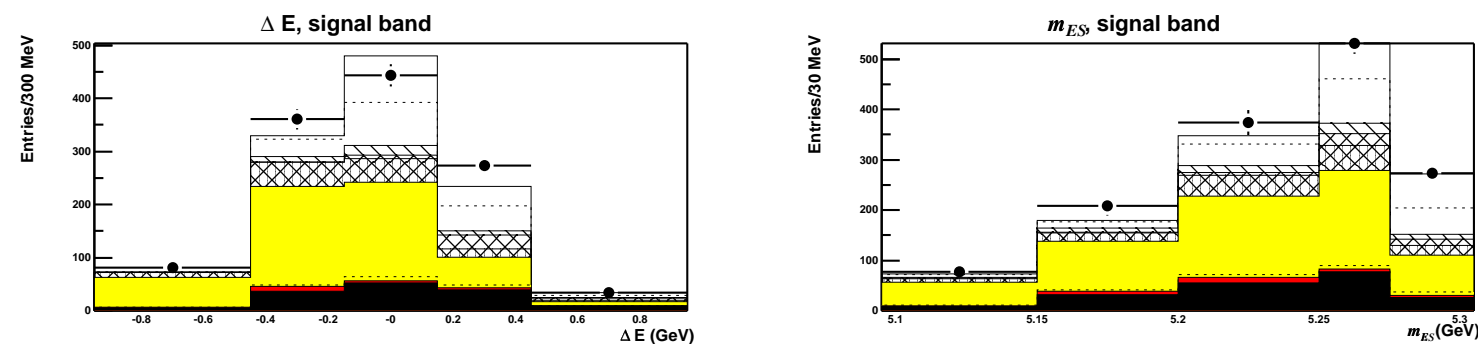

ㅇㅠㄸ

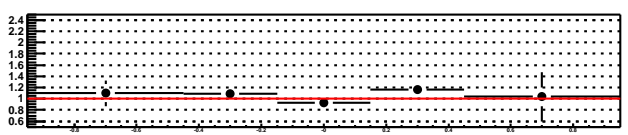

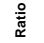
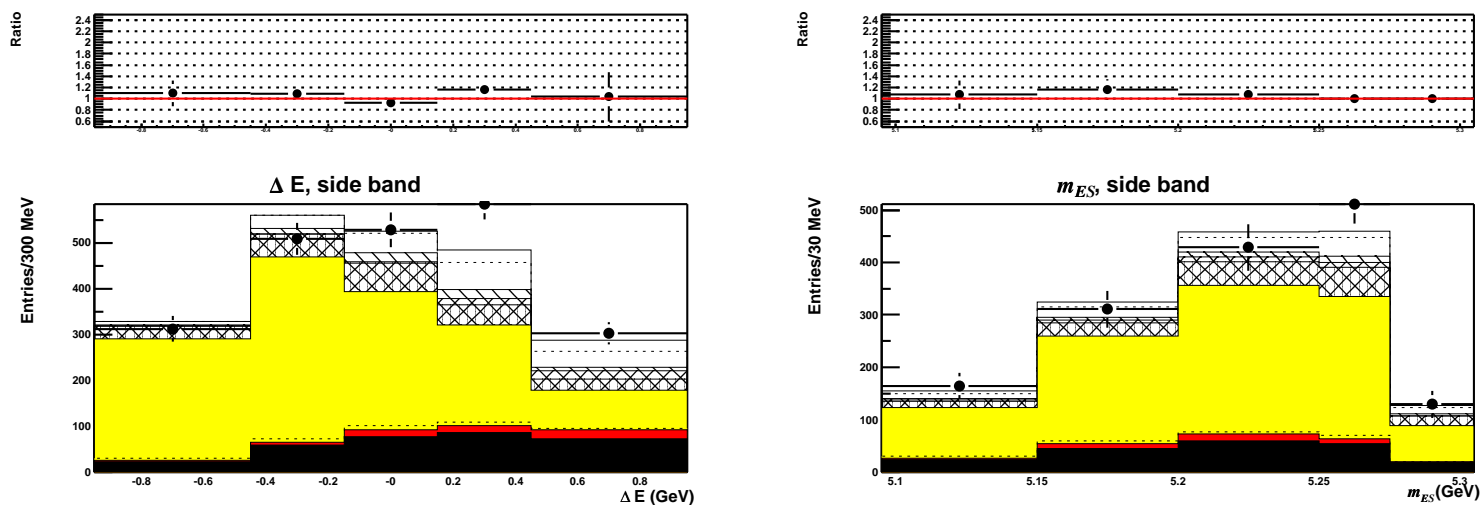

을

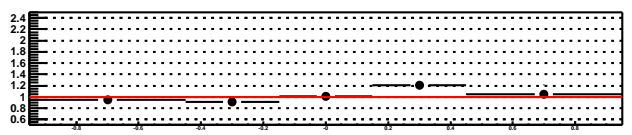

$\stackrel{\circ}{\frac{10}{x}}$

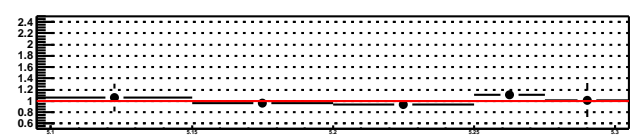

Figure 5.6: $\Delta E$ and $m_{E S}$ projections for $B^{0} \rightarrow \rho^{-} \ell^{+} \nu$. 

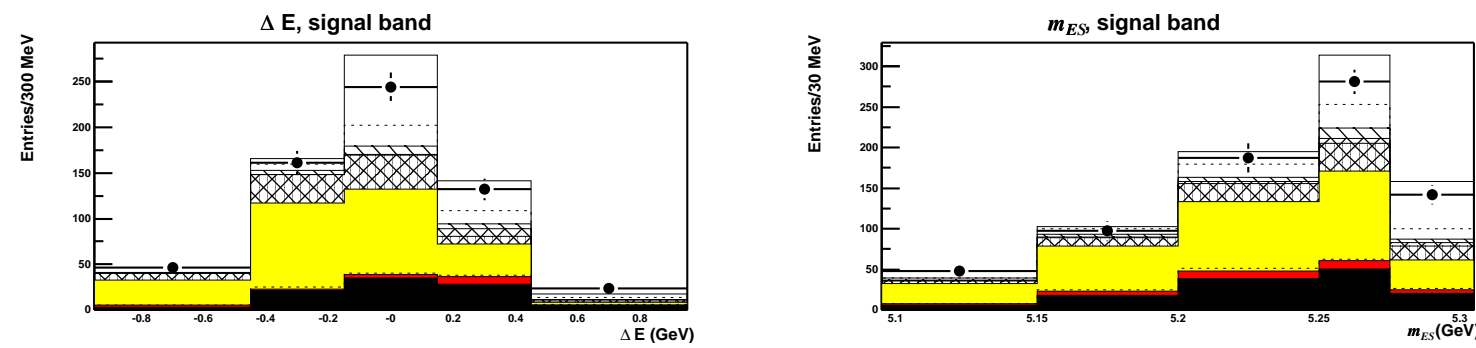

옳

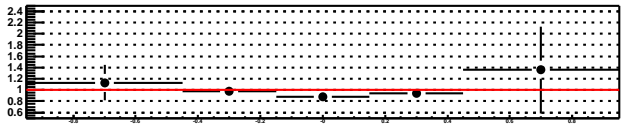

$\frac{\circ}{\frac{10}{x}}$
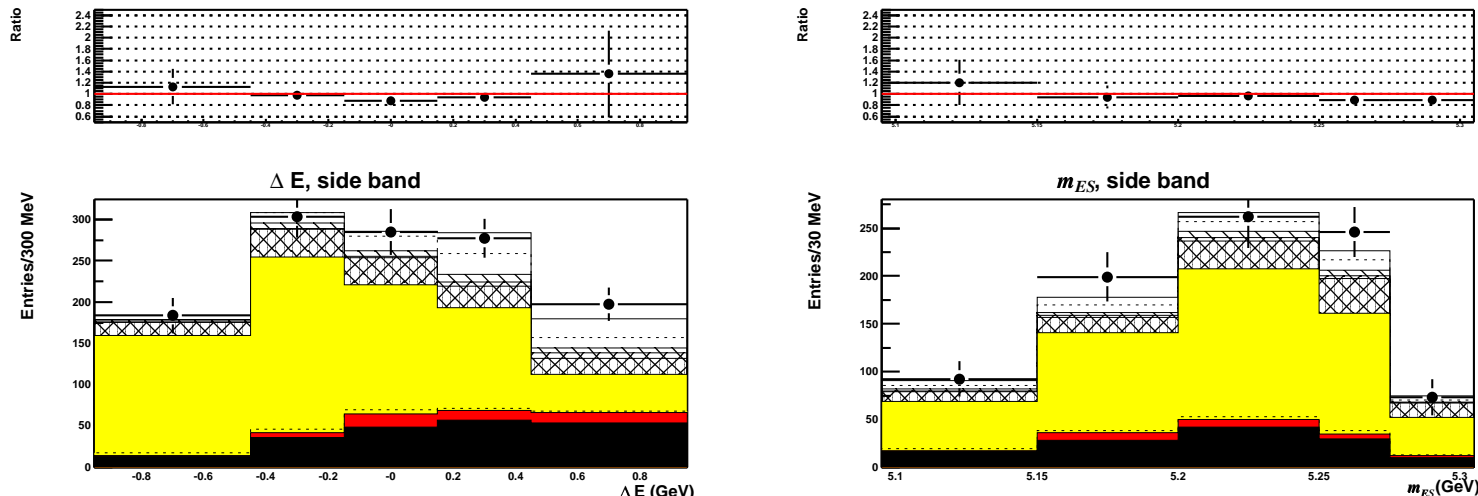

윯

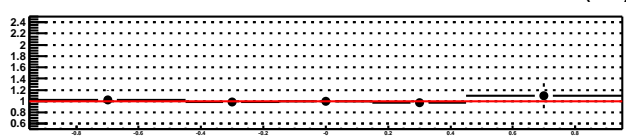

$\stackrel{\circ}{\frac{0}{\pi}}$

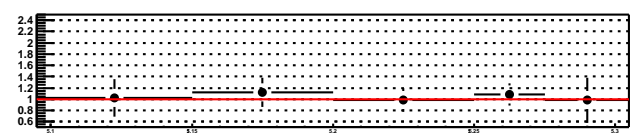

Figure 5.7: $\Delta E$ and $m_{E S}$ projections for $B^{+} \rightarrow \rho^{0} \ell^{+} \nu$.
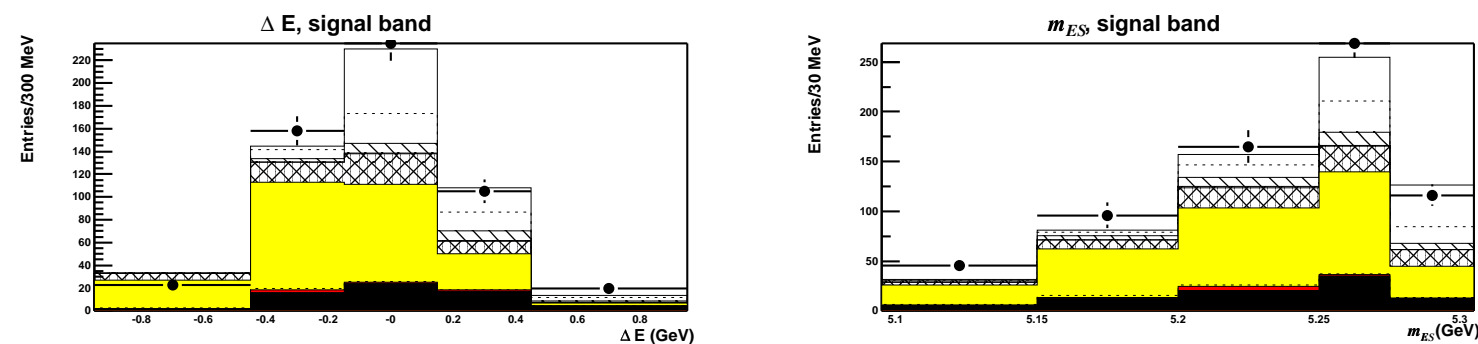

ㅇㅠㄸ

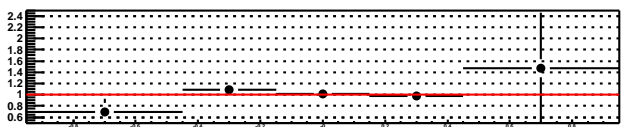

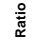
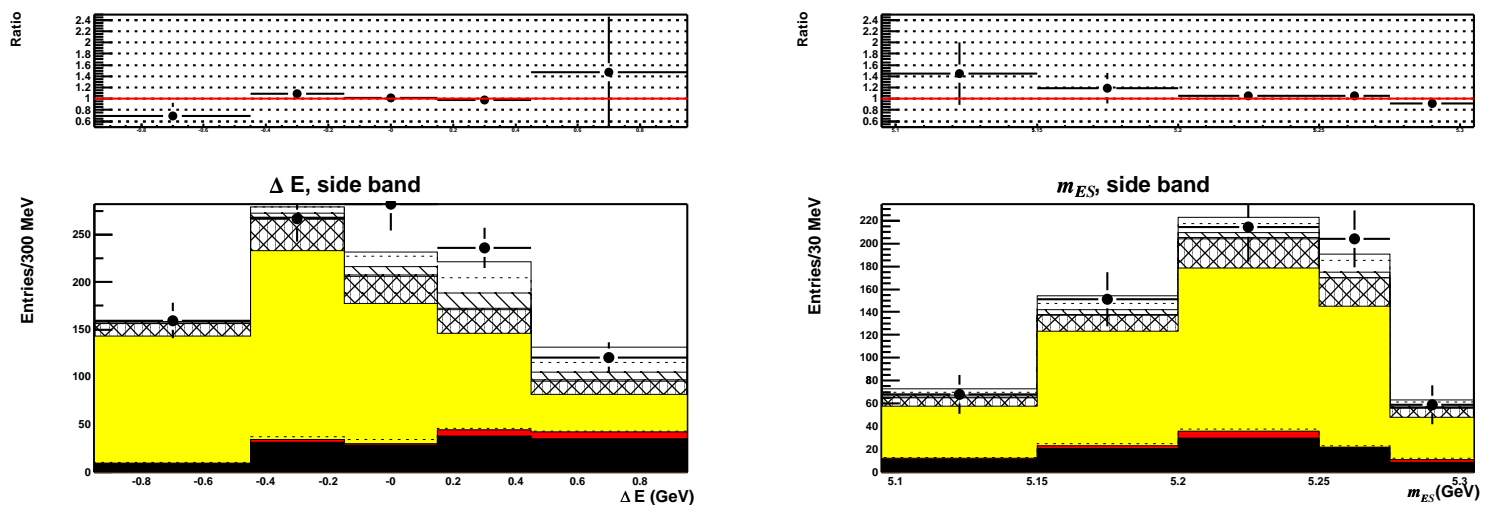

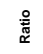

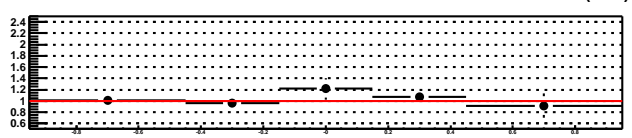

$\stackrel{\circ}{\frac{1}{\pi}}$

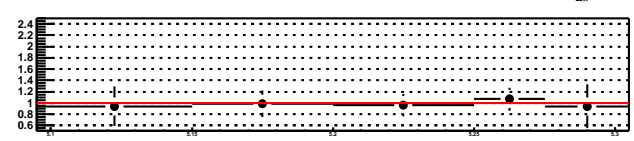

Figure 5.8: $\Delta E$ and $m_{E S}$ projections for $B^{+} \rightarrow \omega^{0} \ell^{+} \nu$. 


\subsubsection{Fit Quality}

To assess the goodness of the fit, the $\chi^{2}$ is measured and a corresponding probability is determined. The value of $\chi^{2}$ is given by

$$
\chi^{2}=\sum_{i} \frac{\left(d_{i}-\sum_{j} p_{j} w_{i j} A_{i j}\right)^{2}}{\sigma_{i}^{2}}
$$

where $d_{i}$ is the number of data events in each bin and $\sigma_{i}$ is the error on the number of data events. In order to avoid the problems associated with low statistics, bins with less than six events are combined with adjacent bins. The number of degrees of freedom, $N_{d o f}$, is equal to the number of bins minus the number of fit parameters. For $N_{d o f}=818, \chi^{2}=801.4$ is found, giving a $\chi^{2}$ probability of 0.62 .

The parameters determined by the fit are used to generate a set of 1000 toy Monte Carlo experiments. The values of $\ln \mathcal{L}_{\text {max }}$ for the experiments are shown in Fig 5.9 along with the value of $\ln \mathcal{L}_{\max }$ determined for the fit to data.

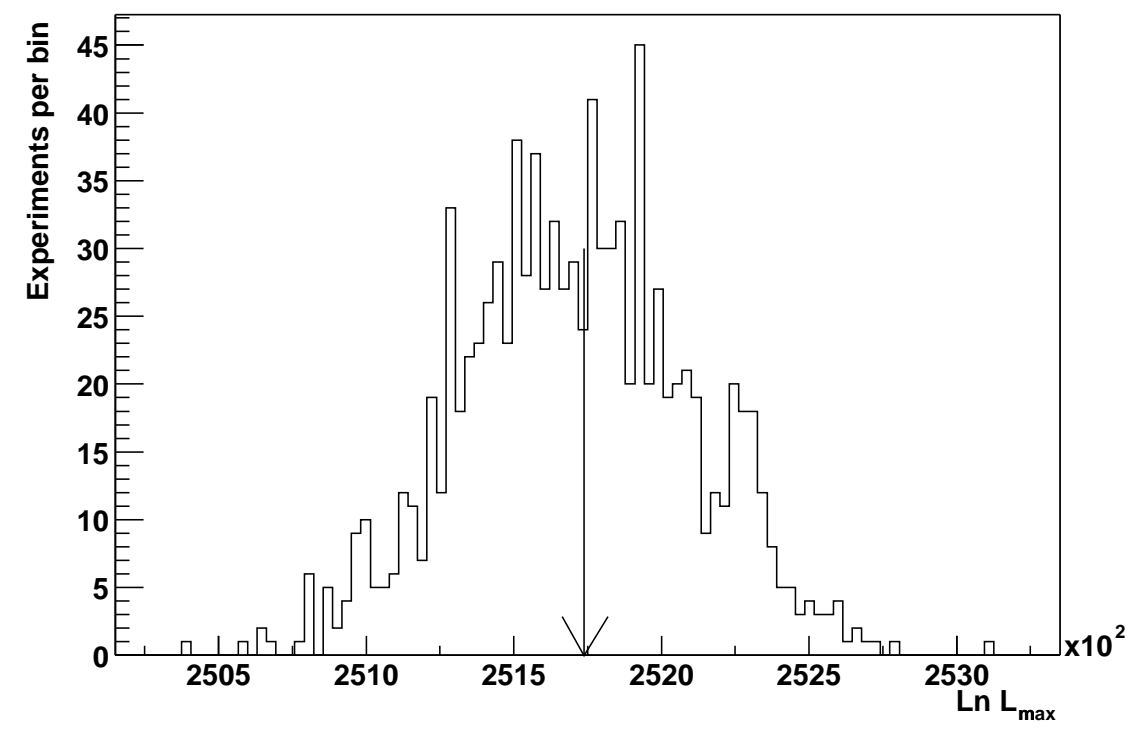

Figure 5.9: The values of $\ln \mathcal{L}_{\text {max }}$ determined from a set of 1000 toy Monte Carlo experiments generated using the parameters determined by the fit to data. The arrow shows the value of $\ln \mathcal{L}_{\max }$ for the fit to data 


\subsubsection{Control Sample Branching Fractions}

In order to check the validity of the analysis procedure the same technique has been applied to determine the branching fractions of the channels employed as the control sample. The primary branching fractions determined are

- $\mathcal{B}\left(B \rightarrow D^{*-} l^{+} \nu\left(\overline{D^{0}} \rightarrow K^{+} \pi^{-}\right)\right)=(5.2 \pm 0.2) \%$

- $\mathcal{B}\left(B \rightarrow D^{*-} l^{+} \nu\left(\overline{D^{0}} \rightarrow K^{+} \pi^{-} \pi^{+} \pi^{-}\right)\right)=(5.2 \pm 0.3) \%$

- $\mathcal{B}\left(B \rightarrow D^{-*} l^{+} \nu\left(\overline{D^{0}} \rightarrow K^{+} \pi^{-} \pi^{0}\right)\right)=(5.7 \pm 0.3) \%$

where the errors are statistical only. These results have not been corrected for the discrepancy in efficiency between the data and Monte Carlo simulation due to the neutrino reconstruction, measured in section 4.12 .3 to be $1.082 \pm 0012$. The most recent $B A B A R$ measurement is $B\left(B \rightarrow D^{*} l \nu\right)=4.69 \pm 0.02 \pm 0.29 \%$ [51]. These results appear in reasonable agreement after taking account of the efficiency discrepancy.

\subsection{Systematics and Checks}

\subsubsection{Lepton PID}

The systematic correction applied to the lepton PID rates is derived from efficiency tables determined from control samples, as described in Sec. 4.11.1. These control samples have associated statistical errors which need to be taken into account. This is achieved by applying a Gaussian smearing (with a width equal to the error on the efficiency table) to the efficiencies for both data and Monte Carlo events. The branching fractions are then recalculated using the new PID weights. The spread of the new branching fractions are $\pm 0.3 \%$ for $\mathcal{B}\left(B \rightarrow \pi^{ \pm} l \nu\right), \pm 0.5 \%$ for $\mathcal{B}\left(B \rightarrow \rho^{ \pm} l \nu\right)$ and $\pm 0.8 \%$ for $\mathcal{B}(B \rightarrow \omega l \nu)$.

The control samples used to determine the PID efficiency tables use low multiplicity events, but the multiplicity in $B$ events is much higher. This is expected to lead 
to a systematic error in the efficiency for lepton identification [52]. A systematic of $1.5 \%$ is assigned. Combining this with the uncertainty due to the statistics of the control sample gives $\pm 1.5 \%$ for $\mathcal{B}\left(B \rightarrow \pi^{ \pm} l \nu\right), \pm 1.6 \%$ for $\mathcal{B}\left(B \rightarrow \rho^{ \pm} l \nu\right)$ and $\pm 1.7 \%$ for $\mathcal{B}(B \rightarrow \omega l \nu)$.

\subsubsection{Fake Lepton Background}

The fake lepton background is determined as detailed in Sec. 4.6.3. The efficiency tables determined from the control samples for each of the particle types have statistical errors. To assess their effect on the fit result the tables are smeared according to their statistical error and the fit is repeated. The spread of the fit results gives $\pm 0.5 \%$ for $\mathcal{B}\left(B \rightarrow \pi^{ \pm} l \nu\right), \pm 0.6 \%$ for $\mathcal{B}\left(B \rightarrow \rho^{ \pm} l \nu\right)$ and $\pm 1.7 \%$ for $\mathcal{B}(B \rightarrow \omega l \nu)$.

The fake background determination is also affected by the particle fractions measured for the hadrons. This is investigated by varying the measured fraction of pions by $5 \%$, the kaons by $15 \%$ and the proton fraction by $25 \%$. This leads to shifts of $\pm 1.0 \%$ for $\mathcal{B}\left(B \rightarrow \pi^{ \pm} l \nu\right), \pm 1.0 \%$ for $\mathcal{B}\left(B \rightarrow \rho^{ \pm} l \nu\right)$ and $\pm 1.5 \%$ for $\mathcal{B}(B \rightarrow \omega l \nu)$. As a further test the fit is repeated with the assumption that all of the fake particles are pions. Under this assumption the fitted branching ratios shift by $-7.0 \%$ for $\mathcal{B}\left(B \rightarrow \pi^{ \pm} l \nu\right),-8.1 \%$ for $\mathcal{B}\left(B \rightarrow \rho^{ \pm} l \nu\right)$ and $-11.2 \%$ for the $\mathcal{B}(B \rightarrow \omega l \nu)$.

The systematic errors due to control sample statistics and hadron fractions are combined to give a systematic error of $\pm 1.1 \%$ for $\mathcal{B}\left(B \rightarrow \pi^{ \pm} l \nu\right), \pm 1.2 \%$ for $\mathcal{B}\left(B \rightarrow \rho^{ \pm} l \nu\right)$ and $\pm 2.3 \%$ for $\mathcal{B}(B \rightarrow \omega l \nu)$.

\subsubsection{Tracking Efficiency}

The systematic errors due to tracking efficiency have been determined in a separate study (the same study used to determine the correction [47]). It is found that each track which uses the BABAR GoodTracksLoose selection (all tracks apart from the $\omega$ daughters) has a systematic error on the efficiency of $0.8 \%$. In the case of the $\omega$ 
daughters which use the GoodTracksVeryLoose selection, the systematic uncertainty is increased to $1.3 \%$ per track.

\subsubsection{Neutrals Correction}

The corrections applied to the photons and $\pi^{0}$ in Monte Carlo events is detailed in section 4.11.2. It is found [46] that each $\pi^{0}$ has an associated systematic error of $5 \%$ on the efficiency. This leads to a systematic error of \pm 1.6 for $\mathcal{B}\left(B \rightarrow \pi^{ \pm} l \nu\right), \pm 2.8$ for $\mathcal{B}\left(B \rightarrow \rho^{ \pm} l \nu\right)$ and \pm 5.0 for $\mathcal{B}(B \rightarrow \omega l \nu)$

\subsubsection{Composition}

$b \rightarrow c l \nu$ Decays

Not all of the branching fractions associated with the $b \rightarrow c l \nu$ background are known to a great precision. In order to assess the systematic uncertainty associated with the $b \rightarrow c l \nu$ composition the individual branching fractions of the constituent decay modes are shifted within the errors given in [7] by assigning weights to these events. The total rate for $b \rightarrow c l \nu$ background is held constant. The shift per mode and resultant shift in the branching fractions are given in the upper section of Table 5.4. Summing the contributions in quadrature gives combined systematic errors of ${ }_{-0.8}^{+0.7} \%$ for $\mathcal{B}\left(B \rightarrow \pi^{ \pm} l \nu\right),{ }_{-2.6}^{+2.7} \%$ for $\mathcal{B}\left(B \rightarrow \rho^{ \pm} l \nu\right)$ and ${ }_{-0.7}^{+0.8} \%$ for $\mathcal{B}(B \rightarrow \omega l \nu)$.

$b \rightarrow u l \nu$ Decays

The $b \rightarrow u l \nu$ branching fraction is fixed to the recent BABAR measurement, $2.24 \pm$ $0.27 \pm 0.26 \pm 0.39 \times 10^{-3}[50]$. To investigate the systematic associated the value is varied by $\pm 20 \%$. The branching ratios shift by ${ }_{-0.11}^{+0.12} \%$ for $\mathcal{B}\left(B \rightarrow \pi^{ \pm} l \nu\right),{ }_{-4.26}^{+3.63} \%$ for $\mathcal{B}\left(B \rightarrow \rho^{ \pm} l \nu\right)$ and ${ }_{-5.14}^{+3.54} \%$ for $\mathcal{B}(B \rightarrow \omega l \nu)$.

As well as the absolute normalisation the composition of the Monte Carlo events is varied as for the $b \rightarrow c l \nu$ background. The variations and the resultant shifts are 


\begin{tabular}{|c|c|c|c|c|}
\hline Decay Mode & $\mathcal{B}$ Variation & $\delta \mathcal{B}(B \rightarrow \pi l \nu) / \mathcal{B}$ & $\delta \mathcal{B}(B \rightarrow \rho l \nu) / \mathcal{B}$ & $\delta \mathcal{B}(B \rightarrow \omega l \nu) / \mathcal{B}$ \\
\hline$B \rightarrow D^{*} l \nu$ & $+10 \%$ & $+0.6 \%$ & $+2.1 \%$ & $-0.6 \%$ \\
$B \rightarrow D^{*} l \nu$ & $-10 \%$ & $-0.7 \%$ & $-2.1 \%$ & $+0.7 \%$ \\
$B \rightarrow D l \nu$ & $+10 \%$ & $-0.3 \%$ & $-1.5 \%$ & $+0.3 \%$ \\
$B \rightarrow D l \nu$ & $-10 \%$ & $+0.3 \%$ & $+1.6 \%$ & $-0.2 \%$ \\
$B \rightarrow c l \nu$ & $+10 \%$ & $-0.2 \%$ & $+0.3 \%$ & $+0.2 \%$ \\
$B \rightarrow c l \nu$ & $-10 \%$ & $+0.1 \%$ & $-0.2 \%$ & $-0.3 \%$ \\
\hline$B \rightarrow \eta l \nu$ & $+50 \%$ & $+0.05 \%$ & $-0.27 \%$ & $-1.58 \%$ \\
$B \rightarrow \eta l \nu$ & $-50 \%$ & $-0.07 \%$ & $+0.32 \%$ & $+1.36 \%$ \\
$B \rightarrow a l \nu$ & $+50 \%$ & $-0.04 \%$ & $-3.9 \%$ & $-0.05 \%$ \\
$B \rightarrow a l \nu$ & $-50 \%$ & $+0.13 \%$ & $+2.8 \%$ & $-0.36 \%$ \\
$B \rightarrow b l \nu$ & $+50 \%$ & $+0.17 \%$ & $+0.77 \%$ & $-2.20 \%$ \\
$B \rightarrow b l \nu$ & $-50 \%$ & $-0.17 \%$ & $-0.88 \%$ & $+1.66 \%$ \\
$B \rightarrow f l \nu$ & $+50 \%$ & $+0.07 \%$ & $-0.05 \%$ & $+0.36 \%$ \\
$B \rightarrow f l \nu$ & $-50 \%$ & $-0.07 \%$ & $+0.09 \%$ & $-0.23 \%$ \\
$B \rightarrow u l \nu$ & $+50 \%$ & $-0.61 \%$ & $+5.24 \%$ & $+7.90 \%$ \\
$B \rightarrow u l \nu$ & $-50 \%$ & $+1.09 \%$ & $-7.44 \%$ & $-10.76 \%$ \\
\hline
\end{tabular}

Table 5.4: $b \rightarrow c l \nu$ (upper) and $b \rightarrow u l \nu$ (lower) branching fraction variations and the associated systematic error. For the $b \rightarrow c l \nu$ decays the $D^{*}$ and $D$ meson decays are shifted independently and then the remainder of the $B \rightarrow c l \nu$ backgound is shifted. For the $b \rightarrow u l \nu$ background the exclusive $\eta, a, b$ and $f$ meson modes are each varied. The remaining $B \rightarrow u l \nu$ background is modelled as non-resonant decays and also varied by $50 \%$. 


\begin{tabular}{|c|c|}
\hline Fit Parameter & $\eta_{i} \pm \Delta \eta_{i}$ \\
\hline$\eta_{1}\left(\mathcal{B}\left(B \rightarrow \pi^{ \pm} \ell \nu\right)\right)$ & $(1.74 \pm 0.09) \times 10^{-4}$ \\
$\eta_{2}\left(\mathcal{B}\left(B \rightarrow \rho^{ \pm} \ell \nu\right)\right)$ & $(2.40 \pm 0.15) \times 10^{-4}$ \\
$\eta_{3}(\mathcal{B}(B \rightarrow \omega \ell \nu))$ & $(1.95 \pm 0.22) \times 10^{-4}$ \\
\hline$\eta_{4}(b \rightarrow c \ell \nu+\pi)$ & $1.09 \pm 0.03$ \\
$\eta_{5}(b \rightarrow c \ell \nu+\rho)$ & $1.17 \pm 0.04$ \\
$\eta_{6}(b \rightarrow c \ell \nu+\omega)$ & $1.12 \pm 0.05$ \\
$\eta_{7}\left(b \rightarrow c \ell \nu+\pi^{0}\right)$ & $0.90 \pm 0.05$ \\
$\eta_{8}\left(b \rightarrow c \ell \nu+\rho^{0}\right)$ & $1.42 \pm 0.06$ \\
\hline
\end{tabular}

Table 5.5: The branching ratios and scale factors determined ignoring Monte Carlo statistics in the fit

given in the lower section of Table 5.4. $\mathcal{B}\left(B \rightarrow \pi^{ \pm} l \nu\right)$ is not significantly affected by the $b \rightarrow u l \nu$ composition, unlike $\mathcal{B}\left(B \rightarrow \rho^{ \pm} l \nu\right)$. The largest variation for the

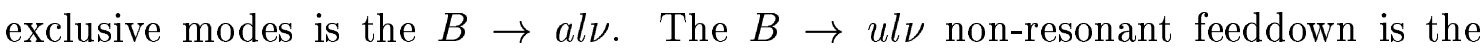
dominant contribution to the systematic error, and is especially pronounced for the vector modes.

The individual deviations are added in quadrature to give the systematic errors: ${ }_{-0.6}^{+1.5} \%$ for $\mathcal{B}\left(B \rightarrow \pi^{ \pm} l \nu\right),{ }_{-9.4}^{+7.0 \%}$ for $\mathcal{B}\left(B \rightarrow \rho^{ \pm} l \nu\right)$ and ${ }_{-12.2}^{+8.9} \%$ for $\mathcal{B}\left(B \rightarrow \omega^{ \pm} l \nu\right)$

\subsubsection{Fitting Systematics}

The fit is designed to accommodate the finite statistics of the Monte Carlo samples. As a cross check the fit is repeated ignoring the statistics of the Monte Carlo events. The results are shown in Table 5.5. As expected the errors from the simple fit are smaller than for the normal fit; they are underestimated. This naive fit is also expected to exhibit a bias [48].

The fit binning is varied, dividing the fit plane into a $5 \times 4$, a $10 \times 10$ array and a more fine binning of the signal region. The effect on the measured branching fractions of all of these configurations are shown in Table 5.6.

As a check the fit is repeated separating the data into electrons and muons. The results are shown in Fig. 5.7. 


\begin{tabular}{|c|c|c|c|}
\hline Systematic Change & $\delta \mathcal{B}\left(B \rightarrow \pi^{ \pm} l \nu\right) / \mathcal{B}$ & $\delta \mathcal{B}\left(B \rightarrow \rho^{ \pm} l \nu\right) / \mathcal{B}$ & $\delta \mathcal{B}(B \rightarrow \omega l \nu) / \mathcal{B}$ \\
\hline 5 by 4 fit plane & $0.5 \%$ & $-1.4 \%$ & $+9.7 \%$ \\
10 by 10 fit plane & $-9.7 \%$ & $-2.9 \%$ & $-13.9 \%$ \\
fine signal region & $-3.4 \%$ & $-0.6 \%$ & $-12.2 \%$ \\
\hline
\end{tabular}

Table 5.6: The impact of different fit binning on the measured branching fractions.

\begin{tabular}{|c|l|l|}
\hline Fit Parameter & \multicolumn{2}{|c|}{$\eta_{i} \pm \Delta \eta_{i}$} \\
\hline & Electrons & Muons \\
$\eta_{1}\left(\mathcal{B}\left(B \rightarrow \pi^{ \pm} \ell \nu\right)\right)$ & $(1.88 \pm 0.15) \times 10^{-4}$ & $(1.40 \pm 0.17) \times 10^{-4}$ \\
$\eta_{2}\left(\mathcal{B}\left(B \rightarrow \rho^{ \pm} \ell \nu\right)\right)$ & $(1.81 \pm 0.25) \times 10^{-4}$ & $(2.45 \pm 0.27) \times 10^{-4}$ \\
$\eta_{3}(\mathcal{B}(B \rightarrow \omega \ell \nu))$ & $(1.35 \pm 0.36) \times 10^{-4}$ & $(1.95 \pm 0.35) \times 10^{-4}$ \\
\hline$\eta_{4}(b \rightarrow c \ell \nu+\pi)$ & $1.19 \pm 0.056$ & $1.06 \pm 0.06$ \\
$\eta_{5}(b \rightarrow c \ell \nu+\rho)$ & $1.22 \pm 0.06$ & $1.22 \pm 0.07$ \\
$\eta_{6}(b \rightarrow c \ell \nu+\omega)$ & $1.17 \pm 0.08$ & $1.09 \pm 0.08$ \\
$\eta_{7}\left(b \rightarrow c \ell \nu+\pi^{0}\right)$ & $0.95 \pm 0.08$ & $0.90 \pm 0.10$ \\
$\eta_{8}\left(b \rightarrow c \ell \nu+\rho^{0}\right)$ & $1.65 \pm 0.11$ & $1.28 \pm 0.10$ \\
\hline
\end{tabular}

Table 5.7: The branching fractions and scale factors determined by the fit for electrons and muon separately.

\subsubsection{Isospin Violation}

In the fit isospin relations are used to constrain $\Gamma\left(B^{0} \rightarrow \rho^{-} l^{+} \nu\right)=2 \Gamma\left(B^{+} \rightarrow \rho^{0} l^{+} \nu\right)$. It has been suggested in a paper by Diaz-Cruz [53] that due to $\rho^{0}-\omega$ mixing and the large $\rho^{0}$ width, this ratio may actually be lowered to $\Gamma\left(B^{0} \rightarrow \rho^{-} l^{+} \nu\right)=$ $1.68 \Gamma\left(B^{+} \rightarrow \rho^{0} l^{+} \nu\right)$. The isospin relation is assumed to hold for the pions. When the new ratio is used in the fit the value of $\mathcal{B}(B \rightarrow \rho l \nu)$ measured is lowered by $8 \%$.

To further investigate the isospin assumptions the fit is modified to return each of the five signal mode branching fractions individually. The results are shown in Table 5.8. The branching fractions determined for the two pion signal modes are close to the expected ratio. The rho signal modes seems to show a large deviation; however the scale factors for the $b \rightarrow c l \nu$ backgrounds for the rho modes also differ. Table 5.9 shows the results of the fit with $\eta_{4}(b \rightarrow c \ell \nu+\pi)=\eta_{7}\left(b \rightarrow c \ell \nu+\pi^{0}\right)$ and $\eta_{5}(b \rightarrow c \ell \nu+\rho)=\eta_{8}\left(b \rightarrow c \ell \nu+\rho^{0}\right)$. The rho mode branching fractions are brought into approximately the ratio expected from isospin. The effect of applying this constraint on the nominal fit setup (with the signal branching fractions constrained 


\begin{tabular}{|c|c|}
\hline Fit Parameter & $\eta_{i} \pm \Delta \eta_{i}$ \\
\hline $\mathcal{B}\left(B^{0} \rightarrow \pi^{-} \ell^{+} \nu\right)$ & $(1.71 \pm 0.13) \times 10^{-4}$ \\
$\mathcal{B}\left(B^{+} \rightarrow \pi^{0} \ell^{+} \nu\right) \times 2 \frac{\tau^{0}}{\tau^{+}}$ & $(1.49 \pm 0.21) \times 10^{-4}$ \\
$\mathcal{B}\left(B^{0} \rightarrow \rho^{-} \ell^{+} \nu\right)$ & $(2.46 \pm 0.26) \times 10^{-4}$ \\
$\mathcal{B}\left(B^{+} \rightarrow \rho^{0} \ell^{+} \nu\right) \times 2 \frac{\tau^{0}}{\tau^{+}}$ & $(1.52 \pm 0.33) \times 10^{-4}$ \\
$\eta_{3}(\mathcal{B}(B \rightarrow \omega \ell \nu))$ & $(1.67 \pm 0.26) \times 10^{-4}$ \\
\hline$\eta_{4}(b \rightarrow c \ell \nu+\pi)$ & $1.13 \pm 0.04$ \\
$\eta_{5}(b \rightarrow c \ell \nu+\rho)$ & $1.19 \pm 0.05$ \\
$\eta_{6}(b \rightarrow c \ell \nu+\omega)$ & $1.13 \pm 0.06$ \\
$\eta_{7}\left(b \rightarrow c \ell \nu+\pi^{0}\right)$ & $0.92 \pm 0.06$ \\
$\eta_{8}\left(b \rightarrow c \ell \nu+\rho^{0}\right)$ & $1.52 \pm 0.09$ \\
\hline
\end{tabular}

Table 5.8: The branching ratios and scale factors determined without isospin constraints

\begin{tabular}{|c|c|}
\hline Fit Parameter & $\eta_{i} \pm \Delta \eta_{i}$ \\
\hline $\mathcal{B}\left(B^{0} \rightarrow \pi^{-} \ell^{+} \nu\right)$ & $(1.76 \pm 0.13) \times 10^{-4}$ \\
$\mathcal{B}\left(B^{+} \rightarrow \pi^{0} \ell^{+} \nu\right) \times 2 \frac{\tau^{0}}{\tau^{+}}$ & $(1.38 \pm 0.21) \times 10^{-4}$ \\
$\mathcal{B}\left(B^{0} \rightarrow \rho^{-} \ell^{+} \nu\right)$ & $(2.12 \pm 0.24) \times 10^{-4}$ \\
$\mathcal{B}\left(B^{+} \rightarrow \rho^{0} \ell^{+} \nu\right) \times 2 \frac{\tau^{0}}{\tau^{+}}$ & $(2.01 \pm 0.32) \times 10^{-4}$ \\
$\eta_{3}(\mathcal{B}(B \rightarrow \omega \ell \nu))$ & $(1.66 \pm 0.26) \times 10^{-4}$ \\
\hline$\left(b \rightarrow c \ell \nu+\left(\pi^{+}+\pi^{0}\right)\right)$ & $1.08 \pm 0.04$ \\
$\left(b \rightarrow c \ell \nu+\left(\rho^{+}+\rho^{0}\right)\right)$ & $1.29 \pm 0.04$ \\
$\eta_{6}(b \rightarrow c \ell \nu+\omega)$ & $1.13 \pm 0.06$ \\
\hline
\end{tabular}

Table 5.9: The branching ratios and scale factors determined without isospin constraints, and setting $\eta_{4}(b \rightarrow c \ell \nu+\pi)=\eta_{7}\left(b \rightarrow c \ell \nu+\pi^{0}\right)$ and $\eta_{5}(b \rightarrow c \ell \nu+\rho)=\eta_{8}\left(b \rightarrow c \ell \nu+\rho^{0}\right)$.

by isospin) is to increase $\mathcal{B}\left(B \rightarrow \pi^{ \pm} l \nu\right)$ by $0.13 \%, \mathcal{B}\left(B \rightarrow \rho^{ \pm} l \nu\right)$ by $-0.81 \%$ and $\mathcal{B}(B \rightarrow \omega l \nu)$ by $-2.50 \%$.

\subsubsection{Non- $B$ Background Scale}

The scale for the non-B background is set by fitting the non- $B$ and fake background to the off-resonance data (see Sec. 5.2.2). To assign a systematic error the level of the non- $B$ background is varied by one sigma. The resulting changes in the branching fractions are $\pm 1.2 \%$ for $\mathcal{B}\left(B \rightarrow \pi^{ \pm} l \nu\right), \pm 0.3 \%$ for $\mathcal{B}\left(B \rightarrow \rho^{ \pm} l \nu\right)$ and $\pm 0.3 \%$ for $\mathcal{B}(B \rightarrow \omega l \nu)$. 


\subsubsection{B Counting}

The number of $B \bar{B}$ decays in the data sample has been determined in a separate study [54]. The number of $\Upsilon(4 S)$ decays is measured by subtracting the scaled number of multihadron events in the off-resonance data sample from the number in the on-resonance sample, corrected for $B \bar{B}$ efficiency. The method has a systematic uncertainty of $\pm 1.1 \%$.

\subsubsection{Ratio of $\tau_{+} / \tau_{0}$}

The ratio of $\tau_{+} / \tau_{0}$ is used to determine the number of each signal mode when the isospin relations are assumed. The value used, $\tau_{+} / \tau_{0}=1.083 \pm 0.017$, is taken from [7]. Varying the value by the given error results in a systematic error of $\pm 0.5 \%$ for $\mathcal{B}\left(B \rightarrow \pi^{ \pm} l \nu\right)$ and $\pm 0.8 \%$ for $\mathcal{B}\left(B \rightarrow \rho^{ \pm} l \nu\right)$ mode.

\subsubsection{Missing Mass Efficiency Corrections}

The efficiency correction primarily due to the missing mass cut, is determined by comparing efficiency of the cut on data and Monte Carlo events in the control samples (Sec. 4.12.3). A measure of the systematic error due to this correction is derived from the spread of the correction factors from each of the three control sample channels. A systematic error of $\pm 1.2 \%$ is assigned.

\subsubsection{Combined Systematic Error}

The individual and combined total systematics errors are shown in Table 5.10. 


\begin{tabular}{|c|c|c|c|}
\hline Systematic & $\delta \mathcal{B}(B \rightarrow \pi l \nu) / \mathcal{B}$ & $\delta \mathcal{B}(B \rightarrow \rho l \nu) / \mathcal{B}$ & $\delta \mathcal{B}(B \rightarrow \omega l \nu) / \mathcal{B}$ \\
\hline Tracking Efficiency & $\pm 1.6 \%$ & $\pm 2.4 \%$ & $\pm 3.4 \%$ \\
Neutral Correction & $\pm 1.6 \%$ & $\pm 2.8 \%$ & $\pm 5.0 \%$ \\
\hline MC Composition & & & \\
$b \rightarrow c l \nu$ & $\pm 0.8 \%$ & $\pm 2.7 \%$ & $\pm 0.8 \%$ \\
$b \rightarrow u l \nu$ & $\pm 1.5 \%$ & $\pm 9.4 \%$ & $\pm 12.2 \%$ \\
\hline Lepton PID & $\pm 1.5 \%$ & $\pm 1.6 \%$ & $\pm 1.7 \%$ \\
Fake leptons & $\pm 1.1 \%$ & $\pm 1.2 \%$ & $\pm 2.3 \%$ \\
B counting & $\pm 1.1 \%$ & $\pm 1.1 \%$ & $\pm 1.1 \%$ \\
B lifetime & $\pm 0.5 \%$ & $\pm 0.8 \%$ & \\
Missing Mass & $\pm 1.2 \%$ & $\pm 1.2 \%$ & $\pm 1.2 \%$ \\
non- $B$ Scale & $\pm 1.2 \%$ & $\pm 0.3 \%$ & $\pm 0.3 \%$ \\
Isospin violation & & $-8 \%$ & \\
\hline Combined & $\pm 4.0 \%$ & ${ }_{-10.8 \%}$ & $\pm 14.0 \%$ \\
\hline
\end{tabular}

Table 5.10: Branching fraction variations and the associated systematic error.

\subsection{Extraction of $\left|\mathrm{V}_{\mathrm{ub}}\right|$}

The CKM element is extracted from the measured branching fractions using the relation

$$
\left|V_{u b}\right|=\sqrt{\frac{\mathcal{B}}{\tilde{\Gamma}_{t h y} \tau_{0}}}
$$

The neutral $B^{0}$ lifetime $\tau_{0}=(1.542 \pm 0.016) \times 10^{-12} \mathrm{~s}$ is taken from [7]. The expected partial width in the abscence of CKM factors $\tilde{\Gamma}_{t h y}$ is model dependent. The signal Monte Carlo used in this analyis is generated using the ISGW2 model [17] which gives,

$$
\begin{aligned}
& \tilde{\Gamma}_{t h y}^{B \rightarrow \pi l \nu}=9.6 \pm 4.8 p s^{-1} \\
& \tilde{\Gamma}_{t h y}^{B \rightarrow \rho l \nu}=14.2 \pm 7.1 p^{-1}
\end{aligned}
$$

Using these values to calculate $\left|V_{u b}\right|$ gives

$$
\begin{aligned}
\left|V_{u b}\right|_{\pi} & =(3.36 \pm 0.11 \pm 0.07 \pm 0.84) \times 10^{-3} \\
\left|V_{u b}\right|_{\rho} & =\left(3.10 \pm 0.14_{-0.21}^{+0.17} \pm 0.76\right) \times 10^{-3}
\end{aligned}
$$

where the errors are statistical, systematic and form factor normalisation respectively. 


\section{Chapter 6}

\section{Conclusions}

\subsection{Introduction}

This chapter summarises the work detailed in this thesis, undertaken between November 2000 up to March 2004. The main body of this work has been the measurement of the branching fractions of the semileptonic exclusive decays $B \rightarrow X_{u} l \nu$, where $X_{u}=\pi^{-}, \pi^{0}, \rho^{-}, \rho^{0}$ or $\omega$. An estimate of $\left|V_{u b}\right|$ has been extracted from the measured branching fractions. The branching fractions and $\left|V_{u b}\right|$ have been determined using the ISGW2 theoretical calculations of the form factors involved. The thesis also presents a method of correcting non-linearities in the response of the EMC readout electronics.

\subsection{EMC Non-linearities}

A number of separate sources of non-linearities have been observed in the EMC readout electronics. A number of corrections have been developed and deployed, but residual non-linearities are still found in areas immediately following switches in amplifier gain. The effect is of the order of $2 \%$ in energy and is present at all three changes in gain range. A method of measuring and correcting the effect in individual channels has been developed and successfully deployed. 


\subsection{Branching Fractions}

A measurement of the branching fractions $\mathcal{B}\left(B^{0} \rightarrow \pi^{-} l^{+} \nu\right), \mathcal{B}\left(B^{0} \rightarrow \rho^{-} l^{+} \nu\right)$ and $\mathcal{B}\left(B^{0} \rightarrow \omega l^{+} \nu\right)$ has been presented. The momentum of the neutrino in the event is reconstructed from the rest of the tracks and clusters in the event. By enforcing certain kinematic constraints we attempt to improve the resolution on the neutrino momentum, but at the expense of signal efficiency. From a sample of $81.9 \mathrm{fb}^{-1}$ the following branching fractions are determined

$$
\begin{aligned}
\mathcal{B}\left(B^{0} \rightarrow \pi^{-} l^{+} \nu\right) & =(1.67 \pm 0.11 \pm 0.07) \times 10^{-4} \\
\mathcal{B}\left(B^{0} \rightarrow \rho^{-} l^{+} \nu\right) & =\left(2.10 \pm 0.18_{-0.28}^{+0.23}\right) \times 10^{-4} \\
\mathcal{B}\left(B^{0} \rightarrow \omega l^{+} \nu\right) & =(1.69 \pm 0.26 \pm 0.24) \times 10^{-4}
\end{aligned}
$$

where the errors are statistical and systematic respectively. These values are determined using the ISGW2 quark model to describe the signal decays.

\section{$6.4\left|\mathrm{~V}_{\mathrm{ub}}\right|$}

From the $B^{0} \rightarrow \pi^{-} l^{+} \nu$ and $B^{0} \rightarrow \rho^{-} l^{+} \nu$ branching fractions determined above, an estimate of $\left|V_{u b}\right|$ has been extracted using the ISGW2 quark model. The theoretical uncertainties for this model are hard to quantify, but the model authors predict an error of around $50 \%$. The measured values of $\left|V_{u b}\right|$ are

$$
\begin{aligned}
& \left|V_{u b}\right|_{\pi}=(3.36 \pm 0.11 \pm 0.07 \pm 0.84) \times 10^{-3}, \\
& \left|V_{u b}\right|_{\rho}=\left(3.10 \pm 0.14_{-0.21}^{+0.17} \pm 0.76\right) \times 10^{-3},
\end{aligned}
$$

where the errors are statistical, systematic and theoretical respectively.

\subsection{Future Work}

The dominant error in the $\left|V_{u b}\right|$ result presented is due to the theoretical modelling of the form factor in the decays. This has been the subject of much activity in the 
theory community and there are now a variety of models available, with varying levels of uncertainties. The modelling of the form factors impact the analysis in two ways. They directly affect the branching fraction measurement when the efficiency of the analysis selection is determined, i.e. through extrapolating from the measured kinematic region to the total rate. Secondly, the theoretical models provide the normalisation required to translate the measured branching fractions into a measurement of $\left|V_{u b}\right|$. This analysis uses only the ISGW2 quark model. A future extension would be to expand the analysis to include other available models. To do this requires implementing each model as a Monte Carlo simulation generator so as to determine the efficiency of the analysis selection. 


\section{References}

[1] S. Menke, "Offline Correction of Non-linearities in the BABAR Electromagnetic Calorimeter Electronics," BABAR Note 527, (private communication).

[2] M. Kobayashi and T. Maskawa, "CP violation in the renormalizable theory of weak interaction," Prog. Theor. Phys. 49 (1973) 652-657.

[3] J. H. Christenson, J. W. Cronin, V. L. Fitch, and R. Turlay, "Evidence for the 2 pion decay of the K(2)0 meson," Phys. Rev. Lett. 13 (1964) 138-140.

[4] A. D. Sakharov, "Violation of CP invariance, C asymmetry, and baryon asymmetry of the universe," Pisma Zh. Eksp. Teor. Fiz. 5 (1967) 32-35.

[5] M. Neubert, "B physics and CP violation," Int. J. Mod. Phys. A11 (1996) 4173-4240, hep-ph/9604412.

[6] BABAR Collaboration, P. F. Harrison and H. R. Quinn, eds., "The BABAR physics book: Physics at an asymmetric B factory,". Papers from Workshop on Physics at an Asymmetric B Factory (BaBar Collaboration Meeting), Rome, Italy, 11-14 Nov 1996, Princeton, NJ, 17-20 Mar 1997, Orsay, France, 16-19 Jun 1997 and Pasadena, CA, 22-24 Sep 1997.

[7] Particle Data Group Collaboration, K. Hagiwara et al., "Review of particle physics," Phys. Rev. D66 (2002) 010001.

[8] J. D. Richman and P. R. Burchat, "Leptonic and semileptonic decays of charm and bottom hadrons," Rev. Mod. Phys. 67 (1995) 893-976, hep-ph/9508250. 
[9] S. Weinberg, "A model of leptons," Phys. Rev. Lett. 19 (1967) 1264-1266.

[10] S. L. Glashow, J. Iliopoulos, and L. Maiani, "Weak interactions with leptons hadron symmetry," Phys. Rev. D2 (1970) 1285-1292.

[11] A. Salam, "Elementary Particle Thory,", p. 367. Almquist and Wiksells, Stockholm, 1969.

[12] N. Cabibbo, "Unitary symmetry and leptonic decays," Phys. Rev. Lett. 10 (1963) 531-532.

[13] L. Wolfenstein, "Parametrization of the Kobayashi-Maskawa matrix," Phys. Rev. Lett. 51 (1983) 1945.

[14] BABAR Collaboration, B. Aubert et al., "Measurement of the CP-violating asymmetry amplitude sin $2 \beta$," Phys. Rev. Lett. 89 (2002) 201802, hep-ex/0207042.

[15] A. Hocker et al., "http://ckmfitter.in2p3.fr/ckm_measure.html,".

[16] N. Isgur, D. Scora, B. Grinstein, and M. B. Wise, "Semileptonic B and D decays in the quark model," Phys. Rev. D39 (1989) 799.

[17] D. Scora and N. Isgur, "Semileptonic meson decays in the quark model: An update," Phys. Rev. D52 (1995) 2783-2812, hep-ph/9503486.

[18] UKQCD Collaboration, L. Del Debbio, J. M. Flynn, L. Lellouch, and J. Nieves, "Lattice-constrained parametrizations of form factors for semileptonic and rare radiative B decays," Phys. Lett. B416 (1998) 392-401, hep-lat/9708008.

[19] P. Ball and V. M. Braun, "Exclusive semileptonic and rare B meson decays in QCD," Phys. Rev. D58 (1998) 094016, hep-ph/9805422.

[20] CLEO Collaboration, R. Fulton et al., "Observations of B meson semileptonic decays to noncharmed final states," Phys. Rev. Lett. 64 (1990) 16-20. 
[21] ARGUS Collaboration, H. Albrecht et al., "Observation of semileptonic charmless B meson decays," Phys. Lett. B234 (1990) 409.

[22] "http://www.slac.stanford.edu/xorg/hfag/semi/summer03-lp/ summer03.shmt1."

[23] CLEO Collaboration, B. H. Behrens et al., "Measurement of B $\rightarrow \rho l \nu$ decay and $\left|\mathrm{V}_{u b}\right|, "$ Phys. Rev. D61 (2000) 052001, hep-ex/9905056.

[24] BABAR Collaboration, B. Aubert et al., "Measurement of the CKM matrix element $\left|V_{u b}\right|$ with charmless exclusive semileptonic B meson decays at BABAR," hep-ex/0207080.

[25] CLEO Collaboration, S. B. Athar et al., "Study of the $q^{2}$ dependence of $B \rightarrow \pi l \nu$ and $B \rightarrow \rho(\omega) l \nu$ decay and extraction of $\left|\mathrm{V}_{u b}\right|, "$ Phys. Rev. D68 (2003) 072003, hep-ex/0304019.

[26] Belle Collaboration, Y. J. Kwon, " $\left|\mathrm{V}_{u b}\right|$ measurements and the related results from Belle,". Prepared for 31st International Conference on High Energy Physics (ICHEP 2002), Amsterdam, The Netherlands, 24-31 Jul 2002.

[27] ALEPH Collaboration, R. Barate et al., "Determination of $\left|V_{u b}\right|$ from the measurement of the inclusive charmless semileptonic branching ratio of $\mathrm{b}$ hadrons," Eur. Phys. J. C6 (1999) 555-574.

[28] OPAL Collaboration, G. Abbiendi et al., "Measurement of $\left|\mathrm{V}_{u b}\right|$ using b hadron semileptonic decay," Eur. Phys. J. C21 (2001) 399-410, hep-ex/0107016.

[29] DELPHI Collaboration, P. Abreu et al., "Determination of $\left|\mathrm{V}_{u b}\right| /|\mathrm{V}(\mathrm{cb})|$ with DELPHI at LEP," Phys. Lett. B478 (2000) 14-30, hep-ex/0105054.

[30] L3 Collaboration, M. Acciarri et al., "Measurement of the inclusive charmless semileptonic branching fraction of beauty hadrons and a determination of $\left|\mathrm{V}_{u b}\right|$ at LEP," Phys. Lett. B436 (1998) 174-186. 
[31] BABAR Collaboration, D. Boutigny et al., "Letter of intent for the study of $\mathrm{CP}$ violation and heavy flavor physics at PEP-II," SLAC-0443.

[32] BABAR Collaboration, "Technical Design Report," SLAC-REP-372.

[33] BABAR Collaboration, B. Aubert et al., "The BaBar detector," Nucl. Instrum. Meth. A479 (2002) 1-116, hep-ex/0105044.

[34] "PEP-II, an Asymmetric B Factory: A Conceptual Design Report," SLAC-R-418.

[35] G. M. Haller and D. R. Freytag, "Analog Floating-Point BiCMOS Sampling Chip and Architecture of the BABAR CsI calormeter Front End Electronics System at SLAC B-Factory," IEEE Trans. Nucl. Sci. 43 (1996) 1610-1614.

[36] D. Best, "Crosstalk in the EMC Front End Electronics," BABAR Note 550, (private communication).

[37] "GEANT, Detector Description and Simulation Tool," CERN Program Library Long Writeup W5013 (1994).

[38] F. De Fazio and M. Neubert, "B $\rightarrow X_{u} l \bar{\nu}$ decay distributions to order $\alpha_{s}$," JHEP 06 (1999) 017, hep-ph/9905351.

[39] Sjöstrand, "PYTHIA 5.7 and JETSET 7.4 Physics Manual," CERN-TH 7112/93 (1993).

[40] G. C. Fox and S. Wolfram, "Event shapes in $e^{+} e^{-}$annihilation," Nucl. Phys. B149 (1979) 413.

[41] U. Langengger et al., "Cut-based Electron Identification," BABAR Note 90.

[42] L. Lista et al., "Muon Identification in the BABAR Experiment," BABAR Note 60 .

[43] C. Zemach, "Three pion decays of unstable particles," Phys. Rev. 133 (1964) B1201. 
[44] BABAR Collaboration, W. T. Ford, "Choice of Kinematic Variables in $B$ Meson Reconstruction - Take 3," BAD Note 53.

[45] F. Di Lodivico et al., "http://www.slac.stanford.edu/BFROOT/www/ Physics/Analysis/AWG/Neutrals/v\%alidation/results/results .html.".

[46] BABAR Collaboration, F. Di Lodovico, "Comparison between Monte Carlo and Data of the $\pi^{0}$ detection and reconstruction efficiency using tau 1 on 1 decays," BAD Note 378.

[47] BABAR Collaboration, E. Varnes, "Report of the Tracking Efficiency Task Force," BAD Note 324.

[48] R. J. Barlow and C. Beeston, "Fitting using finite Monte Carlo samples," Comput. Phys. Commun. 77 (1993) 219-228.

[49] F. James, "Minuit Reference Manual," CERN Program Library Long Writeup D504 (1994).

[50] BABAR Collaboration, D. del Re et al., "Determination of $\mathrm{V}_{u b}$ with inclusive semileptonic $B$ decays," BAD Note 540 .

[51] BABAR Collaboration, B. Aubert et al., "Measurement of $\left|\mathrm{V}_{c b}\right|$ using $B^{0} \rightarrow D^{*} l^{-} \bar{\nu}$ decays," hep-ex/0308027.

[52] BABAR Collaboration, T. Brandt et al., "Measurement of Inclusive Electron Spectrum, Moments and Branching Fractions for Semileptonic B meson Decays," BAD Note 636.

[53] J. L. Diaz-Cruz, G. Lopez Castro, and J. H. Munoz, "Isospin corrections to charmless semileptonic $B \rightarrow V$ transitions," Phys. Rev. D54 (1996) 2388-2390, hep-ph/9605344.

[54] BABAR Collaboration, C. Hearty, "Measurement of the Number of $\Upsilon(4 \mathrm{~S})$ Mesons Produced in Run 1 (B counting)," BAD Note 134. 